\title{
APPLICATIONS OF THE HOMOMORPHIC TRANSFORMATION TO TIME DOMAIN MEASUREMENT PROBLEMS
}

Sedki M. Riad

Norris S. Nahman

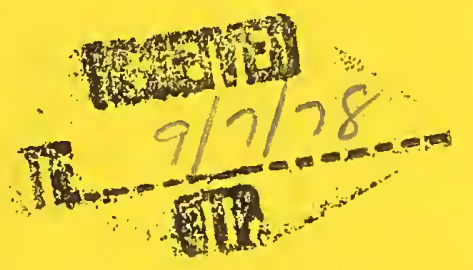

Electromagnetic Technology Division

Center for Electronics and Electrical Engineering

National Engineering Laboratory

National Bureau of Standards

Boulder, Colorado 80303

June 1978 



\section{NBSIR 78-881}

\section{APPLICATIONS OF THE HOMOMORPHIC TRANSFORMATIOU \\ TO TIME DOMMMUN MEASUREMENT PROBLEMS}

Sedki M. Riad

Norris S. Nahman

Electromagnetic Technology Division

Center for Electronics and Electrical Engineering

National Engineering Laboratory

National Bureau of Standards

Boulder, Colorado 80303

June 1978

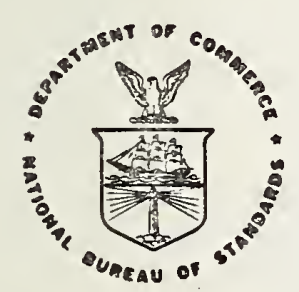

U.S. DEPARTMENT OF COMMERCE, Juanita M. Kreps, Secretary Sidney Harman, Under Secretary Jordan J. Baruch, Assistant Secretary for Science and Technology

NATIONAL BUREAU OF STANDARDS, Ernest Ambler, Director 
The work reported herein was accomplished by Mr. Sedki M. Rlad under a formal agreement between the Dean of the Graduate School of the University of Toledo, Toledo, Ohio, the Dean of the Graduate School of the University of Colorado, Boulder, Colorado, and Dr. Norris S. Nahman of the National Bureau of Standards, Boulder, Colorado, and also Professor, Electrical Engineering Department, University of Colorado, formerly Professor and Chairman, Electrical Engineering Department, University of Toledo. In the course of the work Dr. Nahman provided limited supervision and acted solely as a dissertation advisor. The work was within the National Bureau of Standards Electromagnetics Measurements Graduate Program and was partially funded by contract CST-8392, Devices and Techniques for Time Domain Measurements, Electrical Engineering Department, University of Colorado, Boulder, Colorado. 


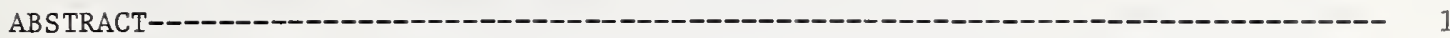

1. INTRODUCTION-

2. THE HOMOMORPHIC TRANSFORMATION--

2.1 Introduction--

2.2 The Homomorphic Transformation--

2.3 The Separation Idea-

2.4 The Homomorphic Deconvolution--

2.5 Computational Realization of the Homomorphic Deconvolution Transform------ 8

2.6 Computational Hint; the Linear Phase Elimination------------------- 9

2.7 Summary---_-

3. THE THEORY AND APPLICATION OF THE HOMOMORPHIC TRANSFORMATION TO DECONVOLUTION IN TIME DOMAIN LINEAR NETWORK ANALYSIS------

3.1 Introduction------

3.2 Time Domain Network Analysis----

3.3 Pure Resistive Networks-

3.3.1 Case of pure resistive line loading------------------------ 22

3.3.2 Commensurate transmission lines---

3.4 Networks with Reactive Components--

3.4.1 Time domain transmission (TDT)------

3.4.2 Time domain reflections (TDR) with reactive loading/unmatched generator impedance--

3.4.2.1 TDR measurements---------

3.4.2.2 Conventiona1 method--- 27

3.4.2.3 The application of the homomorphic deconvolution method--- 27

3.4.2.4 Application-- 28

3.5 Summary and Conclusions-- 5

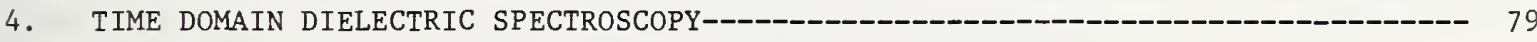

4.1 Introduction--

4.2 Theory of Time Domain Dielectric Spectroscopy---------------------

4.3 The Homomorphic Deconvolution as Applied to TDDS Problems----

4.4 Model for Debye Dielectric- 81

4.5 Application of Homomorphic Deconvolution in TDDS------

4.6 Summary and Conclusions--_-_- 83

5. THE APPLICATION OF THE HOMOMORPHIC DECONVOLUTION TRANSFORM TO THE REMOVAL OF SCATTERING COMPONENTS FROM ANTENNA TEST RANGE DATA----------------------------- 90

5.1 Introduction----

5.2 The Homomorphic Deconvolution as Applied to Antenna System-------------- 91

5.3 The Antenna System-----------------

5.4 The Application---

5.5 Summary and Conclusions- 


\section{CONTENTS (Continued)}

6. TRANSFER NETWORK IDENTIFICATION (MODELING) OF FEED-THROUGH SAMPLING-HEAD------ 118

6.1 Introduction-------- 118

6.2 Theory and Construction of Sampler-_- 118

6.3 Modeling of Physical Structure--- 119

6.4 The Biconical Cavity--- 119

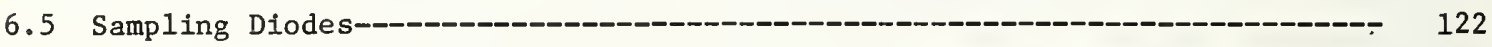

6.5.1 Static measurements $\left(I_{s}\right.$ and $\left.R_{s}\right)--122$

6.5.2 Dynamic measurements $\left(\mathrm{C}_{\mathrm{p}}, \mathrm{L}_{\mathrm{d}}\right.$, and $\left.\mathrm{C}_{\mathrm{j}}\right)-$

6.6 The Sampling Pulse-----

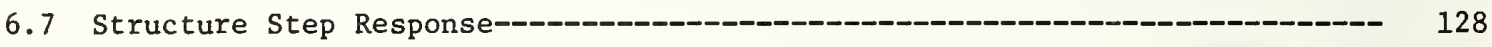

6.8 Sampling Process------------------------------------------------------ 128

6.9 The Effect of the Diode Bias on the Sampling-Head Step Response----------- 130

6.10 The Deconvolution Routine--

6.10.1 The homomorphic deconvolution method---- 131

6.10.2 An application------------- 131

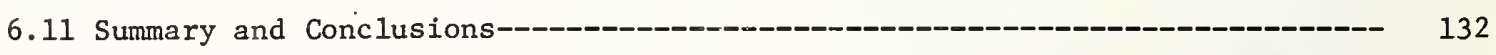

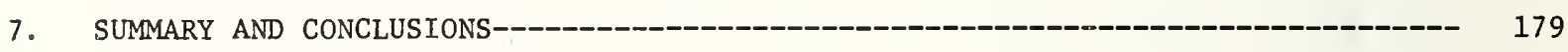

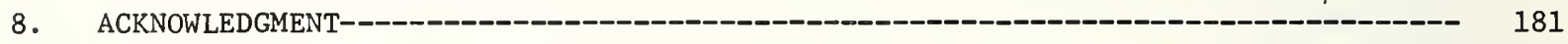

APPENDIX A -- THE Z-TRANSFORM AND THE DISCRETE FOURIER TRANSFORM---------------- 182

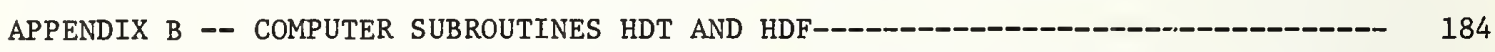

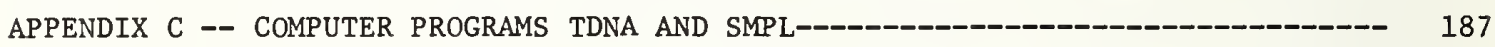

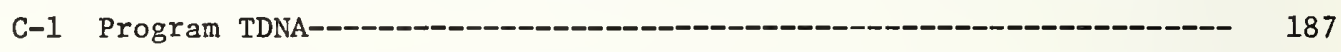

C-2 Program SMPL--_----_-_-_-_- 189

C-3 Subroutines Used in TDNA and SMPL- 191

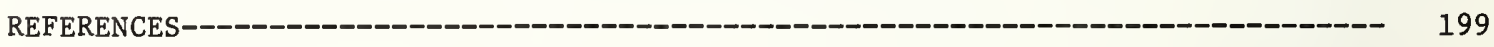


Sedki M. Riad and Norris S. Nahman

This report presents a study of the theory and application of the homomorphic transformation to deconvolution problems occurring in time domain measurements in the picosecond time domain. A homomorphic deconvolution transform was developed and applied successfully to remove the time-windowing restriction required in many time domain measurements. Examples were presented including problems in time domain analysis of linear networks and dielectric spectroscopy, and scattering and multiple reflection in antenna (radiation) systems were considered and treated. Also considered was the development of a model for a 28 picosecond resolution feed-through sampling-head, and the model's step response was computed. Simulation studies were performed using typical input waveforms and the oscilloscope model. The homomorphic transformation was used to deconvolve the model's impulse response from the simulated output. Comparison of the deconvolved output waveforms with the input waveforms showed agreement within the accuracy of the sampled-data simulation.

Key words: Antenna scattering; deconvolution; dielectric spectroscopy; homomorphic transformation; modeling of sampling gates; signal processing; time domain measurements; time domain reflectometry.

\section{INTRODUCTION}

This report is concerned with the study of the theory and application of the homomorphic transformation to deconvolution problems occurring in time domain spectroscopy and scattering. Typical time domain problems that fall under this category include the following: dielectric and magnetic spectroscopy, time domain reflectometry and transmission (network spectroscopy), and wave scattering and muliple reflections in antenna problems. Conventionally, time domain measurements methods are based upon and linited by time-windowing to select the relevant or desired waveform epoch.

In this report a new method is developed based upon the mathematical homomorphic transformation which removes the time-windowing restriction required in the usual time domain measurement method. This allows the use of overlapping time windows which effectively removes the time-window restriction and thus engenders a new method for time domain spectroscopy, and in general, a new method for time domain measurements in the picosecond time domain [1].

Furthermore, because time domain representations have the property of directly exhibiting reflected or scattered contributions, this method has significant impact in the broad class of problems falling under the category of scattering problems which are encountered widely in electrical engineering and in physics [1].

Specifically, this report develops four problems, or applications, which are encountered in various forms in time domain spectroscopy: 
1. Deconvolution of incident and scattered components in the time domain analysis of linear networks.

2. Removal of the time windowing restriction caused by a finite-sized specimen in time domain spectroscopic studies of dielectrics.

3. Removal of scattering components from antenna test range data.

4. Determination (deconvolution) of the excitation waveform from the observed sampled-data response of a picosecond domain sampling oscilloscope.

In summary, this report explores in depth the theory and application of the homomorphic transformation to scattering problems using the above topics as examples, including experimental and computer data reduction studies.

This report is organized into seven chapters and three appendices. The present introduction being the first chapter.

Chapter 2 will be devoted to the study of the theoretical aspects of the homomorphic transformations, and in particular, the homomorphic deconvolution transformation. Also the computational realization of the homomorphic deconvolution transformation will be given.

In chapter 3 the homomorphic deconvolution transform developed in chapter 2 will be applied to various time domain linear network analysis problems. The applications will cover both time domain reflectometry and transmission measurements for pure resistive networks (e.g., commensurate transmission lines network), as well as networks with general (reactive) reflective elements (discontinuities).

Chapter 4 will present the theory and limitations concerning time domain dielectric spectroscopy. The homomorphic deconvolution transformation will be applied to remove the restrictions of the conventional method.

In chapter 5 the homomorphic deconvolution transform will be applied to another class of time domain scattering problems: the removal of scattering components from antenna test range data.

Chapter 6 will be concerned with the transfer network identification (modeling) of feed-through sampling head. The theory and construction of the sampling-head will be reviewed. Various experimental and computational steps involved in the modeling process will be presented. The model will be used to compute the sampling-head step response. A deconvolution routine is to be developed in order to deconvolve the sampling-head's response contribution from the observed waveform on the oscilloscope to get an estimate of the actual waveform at the input port of the oscilloscope.

Chapter 7 will contain the summary and conclusions of the work presented in this report. Relevant detailed mathematical developments and computer programs are referred to in the text and presented in appendices A through $\mathrm{C}$. 


\subsection{Introduction}

It is often encountered in time domain measurements, as well as in other various applications, that an observed signal or a waveform $x(t)$ can be represented by the convolution of two (or more) components $x_{1}(t)$ and $x_{2}(t)$; that is,

$$
x(t)=\int_{-\infty}^{\infty} x_{1}\left(t^{\prime}\right) x_{2}\left(t-t^{\prime}\right) d t^{\prime}
$$

or using the simplified notation

$$
x(t)=x_{1}(t) * x_{2}(t)
$$

where the $(*)$ denotes the convolution process. In the frequency domain, eq. (2.1) takes the form

$$
x\left(e^{j \omega}\right)=x_{1}\left(e^{j \omega}\right) \cdot x_{2}\left(e^{j \omega}\right)
$$

where $x\left(e^{j \omega}\right), x_{1}\left(e^{j \omega}\right)$, and $x_{2}\left(e^{j \omega}\right)$ are the Fourier transforms of $x(t), x_{1}(t)$, and $x_{2}(t)$, respectively, all complex functions of $e^{j \omega}$.

Some of the examples of time domain convolutions are (a) the reflected signal on a transmission line which is the convolution of the incident signal and the impulse response of the reflecting discontinuity and (b) the observed signal using an oscilloscope which is the convolution of the measured signal (at the input gate) and the oscilloscope's impulse response.

In order to separate the components of a convolution, a deconvolution (separation) process is needed. In the past several deconvolution methods were developed for various convolution problems; they all required the knowledge of either one of the convolution components, and in some cases, information about the signals being analyzed. A fairly new method of signal processing, the homomorphic transformation $[2,3,4,5]$, will be described in the present chapter and will be used throughout the following chapters in deconvolving time domain signals. The new method does not require the knowledge of any of the convolution components and suits the class of problems where the frequency domain forms of the convolution components have substantially different rates of variation. As will be shown later, the time domain reflectometry and spectroscopy problems do yield to the new deconvolution method.

Since all signal-processings that will be encountered in the deconvolution process will be carried out using digital computers, the analysis will be presented in the discrete (sampled-data) form. 
A homomorphic system $H$, figure 2.1 , is a nonlinear system, which obeys the generalized principle of superposition $[2,3,4]$; i.e.,

$$
\mathrm{H}\left[\mathrm{x}_{1}(\mathrm{n}) \mathrm{O}_{\mathrm{i}} \mathrm{x}_{2}(\mathrm{n})\right]=\mathrm{H}\left[\mathrm{x}_{1}(\mathrm{n})\right] \mathrm{O}_{\mathrm{o}} \mathrm{H}\left[\mathrm{x}_{2}(\mathrm{n})\right]
$$

and

$$
H\left[c: x_{1}(n)\right]=c \text { i } H\left[x_{1}(n)\right]
$$

where $x_{1}(n)$ and $x_{2}(n)$ are two input sequences to the system $H, n$ is the sequence variable, $c$ is any scalar quantity, $o_{i}$ (or $o_{0}$ ) denotes a rule for combining inputs (or outputs) with each other (e.g., addition, multiplication, convolution, etc.), and : (or i) denotes a rule for combining input (or output) with a scalar. The class of linear systems is a special case for which $O_{i}$ and $O_{0}$ are addition and : and 1 are multiplication. Such systems are said to have an input operation $o_{i}$ and an output one $0_{0}$.

The canonic representation of homomorphic systems is shown in figure 2.2. Both systems $\mathrm{D}_{\mathrm{O}_{i}}$, and $\mathrm{D}_{0}^{-1}$ obey the generalized principle of superposition, eqs. (2.3) and $(2.4)$.

The input operation for $\mathrm{D}_{\mathrm{O}_{i}}$ is $\mathrm{O}_{i}$ while the output one is + (addition); the system $\mathrm{L}$ is a linear system, and the system $\mathrm{D}_{0}^{-1}$ transforms from + to $0_{0}$.

\subsection{The Separation Idea}

Referring to figure 2.2 and letting $x(n)$ denote the combination of the input sequence $x_{1}(n)$ and $x_{2}(n)$, it can be written that

$$
\begin{aligned}
& \mathrm{D}_{0_{i}}[\mathrm{x}(\mathrm{n})]=\mathrm{D}_{\mathrm{O}_{i}}\left[\mathrm{x}_{1}(\mathrm{n}) \mathrm{o}_{i} \mathrm{x}_{2}(\mathrm{n})\right]=\mathrm{D}_{\mathrm{O}_{i}}\left[\mathrm{x}_{1}(\mathrm{n})\right]+\mathrm{D}_{\mathrm{O}_{i}}\left[\mathrm{x}_{2}(\mathrm{n})\right] \\
& =\hat{x}_{1}(n)+\hat{x}_{2}(n) \\
& =\hat{x}(n) \\
& L[\hat{x}(n)]=L\left[\hat{x}_{1}(n)+\hat{x}_{2}(n)\right]=L\left[\hat{x}_{1}(n)\right]+L\left[\hat{x}_{2}(n)\right] \\
& =\hat{y}_{1}(n)+\hat{y}_{2}(n) \\
& =\hat{y}(n) \\
& D_{0}^{-1}[\hat{y}(n)]=D_{0}^{-1}\left[\hat{y}_{1}(n)+\hat{y}_{2}(n)\right]=D_{0}^{-1}\left[\hat{y}_{1}(n)\right] 0_{0} D_{0}^{-1}\left[\hat{y}_{2}(n)\right] \\
& =y_{1}(n) 0_{0} y_{2}(n) \\
& =y(n) \text {. }
\end{aligned}
$$


Now, if it is required to recover one of the input signals $\left(\varepsilon_{\cdot} g ., x_{1}(n)\right)$ from the combined sequence $x(n)$, the linear system $L$ must have the filtering property

$$
L\left[\hat{x}_{1}(n)+\hat{x}_{2}(n)\right]=\hat{x}_{1}(n) ;
$$

i.e.,

$$
\hat{y}(n)=\hat{x}_{1}(n) \text {. }
$$

Then, choosing

$$
\mathrm{D}_{\mathrm{O}_{0}}=\mathrm{D}_{\mathrm{O}_{\mathrm{i}}}
$$

eqs. (2.7) and (2.5) yield

$$
y(n)=D_{0_{i}}^{-1}\left[\hat{x}_{1}(n)\right]=x_{1}(n) .
$$

In conclusion, the separation of the combined sequences $x_{1}(n)$ and $x_{2}(n)$ depends on the success in separating their transforms $\hat{x}_{1}(n)$ and $\hat{x}_{2}(n)$, which depends in turn on the properties of the signals involved.

\subsection{The Homomorphic Deconvolution}

As mentioned earlier in section 2.1, the deconvolution is the process of separating convoluted signals from each other. The homomorphic deconvolution is then the use of a suitable homomorphic system (of transformations) to separate (deconvolve) a convolution. The input and output operations of a homomorphic deconvolution system are the convolution operation (*), according to eq. (2.9). The canonic form of a homomorphic deconvolution system is shown in figure 2.3. In the figure, the transform $D_{*}$ converts the convolution operation into an addition. The transform $D_{*}^{-1}$ is the inverse transform of $D_{*}$. In constructing the system $D_{*}$ to do its function, there are two useful mathematical operations that will be used:

a. The $z$-transform $z[]$, appendix A, converts a convolution into a product, eq. (A.13). In other words, the z-transform is a homomorphic transform with (*) and $(\cdot)$ as the input and output operations, respectively.

b. The complex logarithm operator log converts a product into a sum; i.e., the $10 \mathrm{~g}$ is a homomorphic transform with $(\cdot)$ and $(+)$ as the input and output operations, respectively.

The simplest form for the $D_{*}$ transform can then be the cascade combination of the $z-$ transform and the complex logarithm, figure 2.4a, but if it is preferable in the separation process to deal with sequences rather than their z-transforms, the addition of an inverse $z$-transform $z^{-1}[]$, eq. (A.3), which is a linear one, will not alter the output operation, figure $2.4 \mathrm{~b}$. 
Studying the signal flow in the constructed system $D_{*}$ of figure 2.4 , with $x(n)$ as the convolution of two sequences $x_{1}(n)$ and $x_{2}(n)$, the following relations are obtained:

$$
\begin{aligned}
x(z) & =z[x(n)] \\
& =z\left[x_{1}(n) * x_{2}(n)\right] \\
& =z\left[x_{1}(n)\right] \cdot z\left[x_{2}(n)\right] \\
& =x_{1}(z) \cdot x_{2}(z) \\
\hat{x}(z) & =10 g[x(z)] \\
& =\log \left[x_{1}(z) \cdot x_{2}(x)\right] \\
& =10 g\left[x_{1}(z)\right]+10 g\left[x_{2}(z)\right] \\
& =\hat{x}_{1}(z)+\hat{x}_{2}(z) \\
\hat{x}(\hat{n}) & =z^{-1}\left[\hat{x}_{(z)}(z)\right. \\
& =z^{-1}\left[\hat{x}_{1}(z)+\hat{x}_{2}(z)\right] \\
& =z^{-1}\left[\hat{x}_{1}(z)\right]+z^{-1}\left[\hat{x}_{2}(z)\right] \\
& =\hat{x}_{1}(\hat{n})+\hat{x}_{2}(\hat{n}) \cdot
\end{aligned}
$$

The reason for denoting the sequence variable in eq. (2.13) by $\hat{n}$ is to distinguish the new domain from the original n-domain. This $\hat{n}$-domain is a nonphysical domain; it is a pure mathematical one or what is to be called the hypothetical n-domain. If the n-domain is the time domain, then the $\hat{n}$-domain is to be referred to as the hypothetical time domain. The signal $\hat{x}(\hat{n})$ in the hypothetical $\hat{n}$-domain is historically named the cepstrum (reversing the order of the first four letters of the word spectrum).

The separation process will then be achieved using linear filters in the $\hat{n}$-domain to split $\hat{x}(\hat{n})$ into its components $\hat{x}_{1}(\hat{n})$ and $\hat{x}_{2}(\hat{n})$. The filtration problem will be greatly simplified if $\hat{x}(\hat{n})$ is real. Assuming that both $x(n)$ and $\hat{x}(\hat{n})$ are real stable sequences is not in fact a restriction since these are the properties of the majority of signals encountered in this work.* Besides, simple mathematical operations can change the stability conditions of a given signal. For example, exponential weighting can be used to secure stability as being discussed in appendix $A$. This assumption implies that the regions of convergence of both $X(z)$ and $\hat{X}(z)$ must include the unit circle, see appendix A.

On the unit circle $z=e^{j \omega}, \hat{X}(z)$ can be expressed as $\hat{X}\left(e^{j \omega}\right)$,

$$
\hat{x}\left(e^{j \omega}\right)=\hat{x}_{R}\left(e^{j \omega}\right)+\hat{x}_{I}\left(e^{j \omega}\right)
$$

\footnotetext{
* Here a stable signal is defined as one whose z-transform contains no poles exterior to or on the unit circle. Note that this definition corresponds to a stable signal as being defined as one whose Laplace or s-transform contains no poles on the imaginary axis or in the right-hand plane. 
where the subscripts $R$ and I refer to real and imaginary components of a complex quantity. Since $\hat{x}(\hat{n})$ is assumed to be real, $\hat{x}_{R}\left(e^{j \omega}\right)$ and $\hat{x}_{I}\left(e^{j \omega}\right)$ must respectively be even and odd periodic functions of $\omega$ with period $2 \pi$. Both functions must be analytic (continuous functions of $\omega$ ) to meet the stability requirements. Since $\hat{X}=\log x$ by virtue of eq. (2.12), the analyticity of the complex logarithmic function is to be studied.

The complex logarithmic function $\log$ is defined as

$$
\begin{aligned}
\log X & =\log |x|+j \arg [X] \\
& -\pi<\arg [X] \leq+\pi
\end{aligned}
$$

Using eqs. (2.12) and (2.14), together with the above definition, the real and imaginary components of $\hat{X}\left(e^{j \omega}\right)$ are

$$
\hat{x}_{R}\left(e^{j \omega}\right)=\log \left|x\left(e^{j \omega}\right)\right|
$$

and

$$
\hat{\mathrm{x}}_{I}\left(e^{j \omega}\right)=\arg \left[X\left(e^{j \omega}\right)\right] .
$$

The real part, eq. (2.16a), is analytic as long as the $z$-transform $X(z)$ does not have any zeros on the unit circle. However, the exponential weighting method, appendix $A$, can be used to move any zeros lying on the unit circle to make eq. (2.16a) analytic. The imaginary part, eq. (2.16b), is not a continuous function according to eq. (2.15b); this does have two undesired effects:

a) The eq. (2.12c) will not (always) be true. Using eq. (2.14), eq. (2.12) becomes

$$
\begin{aligned}
& \log |x|=\log \left|x_{1}\right|+\log \left|x_{2}\right| \\
& \arg [x]=\arg \left[x_{1}\right]+\arg \left[x_{2}\right]
\end{aligned}
$$

where the three arguments in eq. (2.17b) are all defined in the region $-\pi$ to $+\pi$. The relation of eq. (2.17a) is always true, but eq. (2.17b) is not because of the restrictions on the arguments.

b) $\hat{x}(z)$ will not (always) be a valid z-transform because of the noncontinuity of the imaginary component of $\hat{\mathrm{X}}(=\log \mathrm{X})$, thus invalidating eq. (2.13).

The solution to the complex logarithm problem is simple; it is to replace the discontinuous complex logarithm by the continuous complex logarithm clog defined as

$$
\begin{aligned}
& \operatorname{clog}[X]=\log |x|+j \operatorname{carg}[X] \\
& \operatorname{carg}[X]=\arg [X]+2 m \pi
\end{aligned}
$$

where carg is the continuous argument function. This new function is formed by reconstructing the argument function to be a continuous one by addding (or subtracting) integer multiples of $2 \pi$, figure 2.5 . 
In conclusion, for the system $D_{*}$ to function properly (i.e., convert the real stable convolution $x(n)$ into a real stable sum $x(n)$ ), the constructed clog function must be used instead of the $\log$ function; and the real and imaginary components of both $x\left(e^{j \omega}\right)$ and $\hat{\mathrm{X}}\left(\mathrm{e}^{\mathrm{j} \omega}\right)$ must be respectively even and odd continuous periodic functions of $\omega$ with period $2 \pi$.

The inverse system $D_{*}^{-1}$ should perform the reverse processes of the system $D_{*}$; i.e., convert an addition at the input to a convolution at the output. $\mathrm{D}_{*}^{-1}$ is constructed by reversing the order of the transforms of $D_{*}$ replacing each transform by its inverse, figure 2.6. The system $D_{*}^{-1}$ does not have any ununiqueness problems. Since the system $\mathrm{D}_{*}^{-1}$ is the inverse transform of $D_{*}$,

$$
D_{*}^{-1}\left[D_{*}[x(n)]\right]=x(n) .
$$

Since both $x(n)$ and $\hat{x}(\hat{n})$ are assumed to be real and stable, it follows that both $\hat{y}(\hat{n})$ and $y(n)$ are both real and stable. Thus the regions of convergence of both $Y(z)$ and $\hat{Y}(z)$ must include the unit circle.

\subsection{Computational Realization of the Homomorphic Deconvolution Transform}

In most physical situations the observed signals have to be dealt with numerically rather than analytically. The digital computer is often used to perform various kinds of numerical processing on such signals. For the separation of physical convolution signals, a digital computer version of the homomorphic deconvoluting transform has to be derived.

In section 2.4 , it was decided that the z-transforms $X(z), \hat{X}(z), \hat{Y}(z)$, and $Y(z)$ have to include the unit circle in their regions of convergence. Thus the inverse $z$-transforms can then be calculated using the unit circle as the integration contour, eq. (A.3). Accordingly, the evaluation of the above mentioned z-transforms is only needed on the unit circle; i.e., it is employed to calculate the signal Fourier transforms rather than the $z$-transforms, eq. (A.6). Because of the discreteness and finiteness required in numerical computational methods, the input signal has to be used in its discrete version. The discrete Fourier transform, figure 2.7, shows the computational realization of the homomorphic deconvoluting transform, in which the signals are related as follows:

$$
\begin{aligned}
& x(k)=\left.x\left(e^{j \omega}\right)\right|_{\omega}=2 \pi k / N \\
&=\sum_{n=0}^{N-1} x(n) e^{-j(2 \pi k / N) n} \\
& \hat{x}(k)=\operatorname{clog}[x(k)] \\
&=\log |x(k)|+j \operatorname{carg}[x(k)], \\
& \operatorname{carg}[x(k)]=\arg [x(k)]+m 2 \pi .
\end{aligned}
$$


Here m may be zero or a positive or negative integer, such that $\operatorname{carg}[\mathrm{X}(\mathrm{k})]$ is continuous.

$$
\begin{aligned}
& \hat{x}(\hat{n})=\frac{1}{N} \sum_{k=0}^{N-1} \hat{x}(k) e^{j(2 \pi k / N) \hat{n}} \\
& \hat{y}(\hat{n})=L[\hat{x}(\hat{n})] \\
& \hat{Y}(k)=\sum_{n=0}^{N-1} \hat{y}(\hat{n}) e^{-j(2 \pi k / N) \hat{n}} \\
& Y(k)=\exp [\hat{Y}(k)] \\
& y(n)=\frac{1}{N} \sum_{k=0}^{N-1} Y(k) e^{j(2 \pi k / N) n} .
\end{aligned}
$$

It has to be noted. that the input sequence $x(n)$ is the convolution of two sequences $x_{1}(n)$ and $x_{2}(n)$ both of finite number of samples $N$; consequently, the cepstrum $\hat{x}(\hat{n})$ is the sum of the two contributions $\hat{x}_{1}(\hat{n})$ and $\hat{x}_{2}(\hat{n})$ due to $x_{1}(n)$ and $x_{2}(n)$, respectively. The output of the linear filter $\hat{y}(\hat{n})$ should be equal to either of the components $\hat{x}_{1}(\hat{n})$ or $\hat{x}_{2}(\hat{n})$ to yield a system output $y(n)$ which correspondingly equals either $x_{1}(n)$ or $x_{2}(n)$. Also, it is to be noted that all the sequences involved in the transform equations, eqs. (2.19) through (2.25), either in the $n, k$, or $\hat{n}$-domains are each of a finite number of samples $N$, and they each satisfy the relation

$$
h(m)=h(m+r N) ; r \text { integer; }
$$

i.e., all the computations need to be done only for sequence variables ranging between 0 and $\mathrm{N}-1$.

The system of figure 2.7 was simulated by the computer subroutine HDT given in appendix B. In cases where the input and output signals to the deconvolution routine are represented by their discrete Fourier transforms, the subroutine HDF is to be used. HDF is the same as HDT except that it does not contain the first DFT and the last DIFT.

\subsection{Computational Hint; the Linear Phase Elimination}

Let the sequence $x(n)$, the input to the homomorphic deconvolution transform of figure 2.7 , be the result of delaying another sequence $s(n)$ by $n_{0}$ samples; i.e.,

$$
x(n)=s\left(n-n_{0}\right)
$$


Following the transform equations, eqs. (2.19), (2.20), and (2.21), it can be written that

$$
\begin{aligned}
& x(k)=S(k) \cdot e^{-j(2 \pi k / N) n_{0},}, \\
& \hat{X}(k)=\hat{S}(k)-j(2 \pi k / N) n_{0},
\end{aligned}
$$

and

$$
\hat{x}(\hat{n})=\hat{s}(\hat{n})+(-1)^{\hat{n}+1} n_{0} / n
$$

The second term in eq. (2.30) is of the form of a decaying oscillation. This term is called the linear phase term because it is due to the linear phase component in the Fourier transforms, eqs., (2.28) and (2.29). Assuming that it is desired to perform linear filtering on $\hat{s}(\hat{n})$, the linear phase term must be removed. Said another way, $\hat{s}(\hat{n})$ contains the desired information while the addition of the linear phase term obscures this information. Accordingly, the linear phase term of $\hat{x}(\hat{n})$ must be removed. Figure 2.8 illustrates how the linear phase term distorts the information $\hat{s}(\hat{n})$ contained in $\hat{x}(\hat{n})$.

The existence of such a linear phase term also contradicts the requirements of having odd periodic continuous phase (argument) function with $\omega$. This linear phase component has to be eliminated, and the best place to perform the elimination is right after taking the clog of the DFT of the signal. Figure 2.9 shows the linear phase elimination process.

\subsection{Summary}

This chapter started with a review of the convolution relations in both the time and frequency domains, and the need for deconvolution (separation) techniques.

The general class of the homomorphic systems was defined, and their properties were stated showing how they can be used for signal separations. In particular, the homomorphic deconvolution transform was developed. The related problems, such as the nonanalyticity of the complex logarithmic function and the effect of linear phase component, were presented and discussed, and solutions to such problems were given.

The computational realization of the deconvolution transformation was made possible in the form of digital computer subroutines HDT and HDF.

In the next chapters, the new deconvolution method will be put into use in deconvolving various kinds of time domain signals, such as those observed in time domain spectroscopy measurements. 


$$
x(n)=x_{1}(n) O_{i} x_{2}(n) \stackrel{0_{i}}{\longrightarrow} \begin{aligned}
& 0_{0} \longrightarrow y(n)=H[x(n)] \\
&=H\left[x_{1}(n)\right] 0_{0} H\left[x_{2}(n)\right]
\end{aligned}
$$

Figure 2.1 A Homomorphic System $H$ with $O_{i}$, and $O_{0}$ as the Input and Output Operations, Respectively.

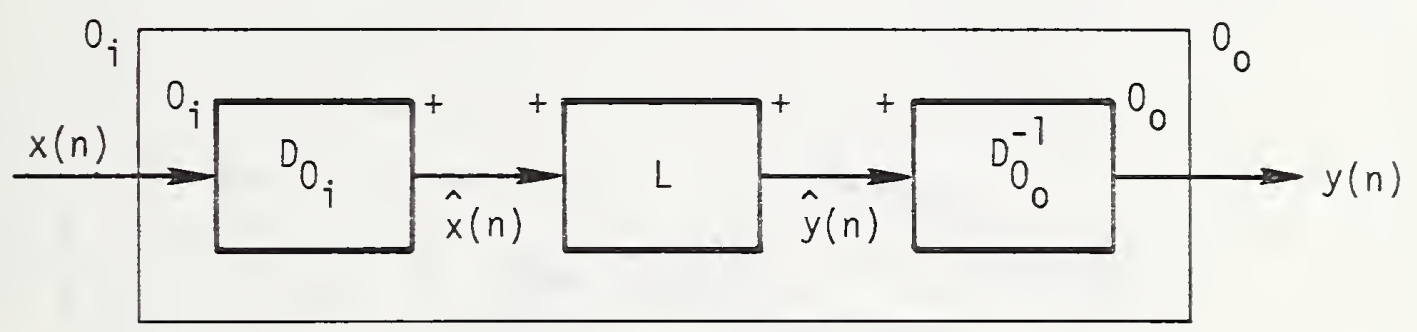

Figure 2.2. Canonic Representation of Homomorphic Systems.

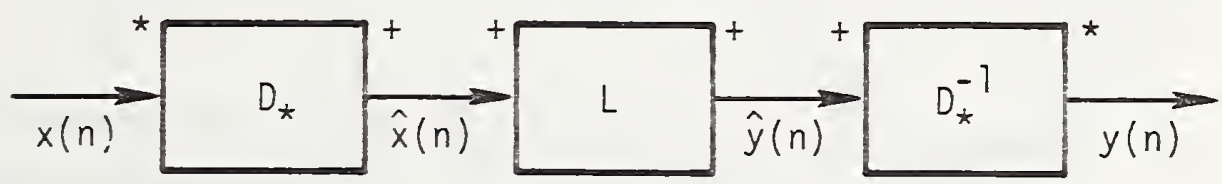

Figure 2.3. The Canonic Form of a Homomorphic Deconvolution system. 


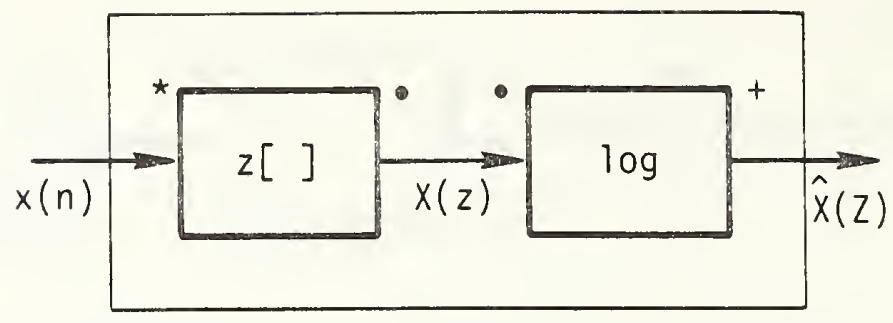

a. The Output signal is a z-Transform.

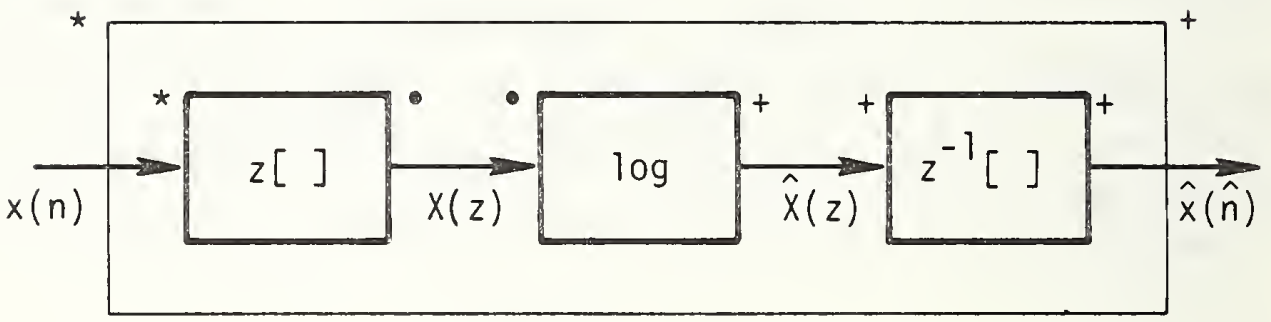

b. The Output signal is a Sequence.

Figure 2.4. Possible Forms for Construction of the Transform $D_{*}$, a Homomorphic system with (*) as the Input Operation and (+) as the output operation. 
$\arg \left[x\left(e^{j \omega}\right)\right]$
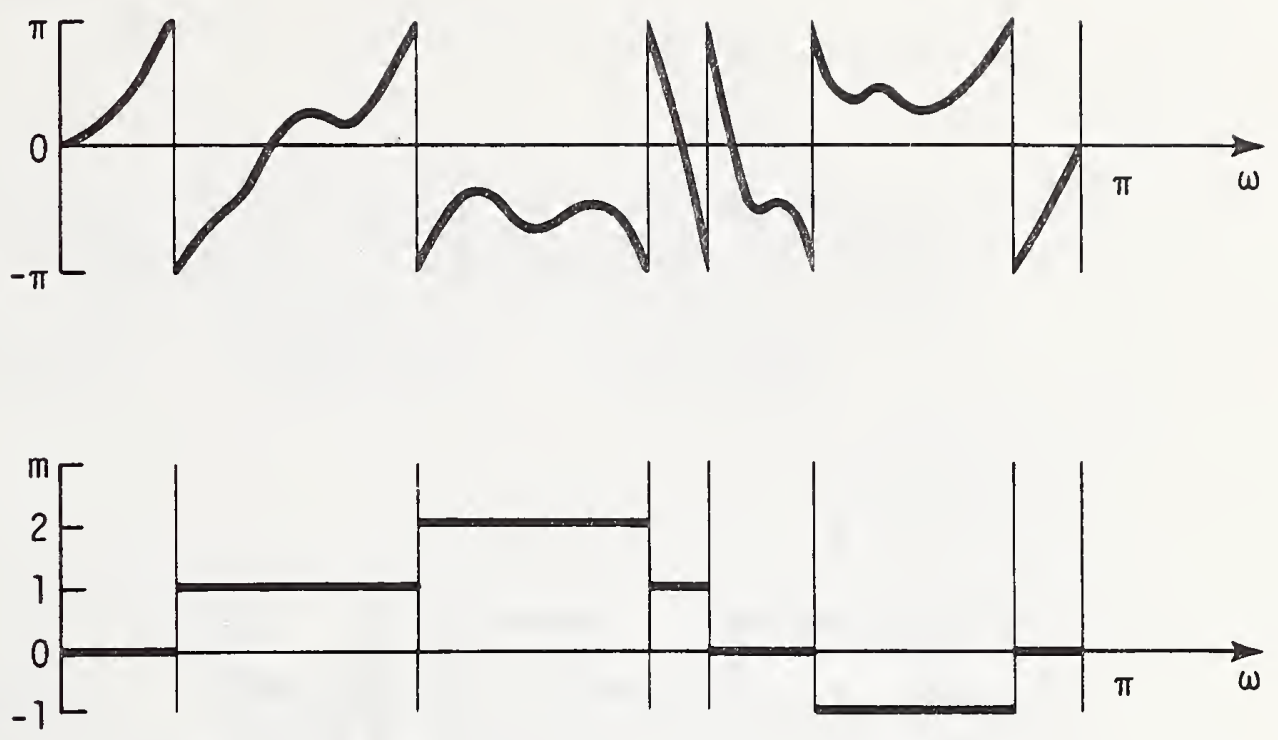

$\operatorname{carg}\left[x\left(e^{j \omega}\right)\right]$

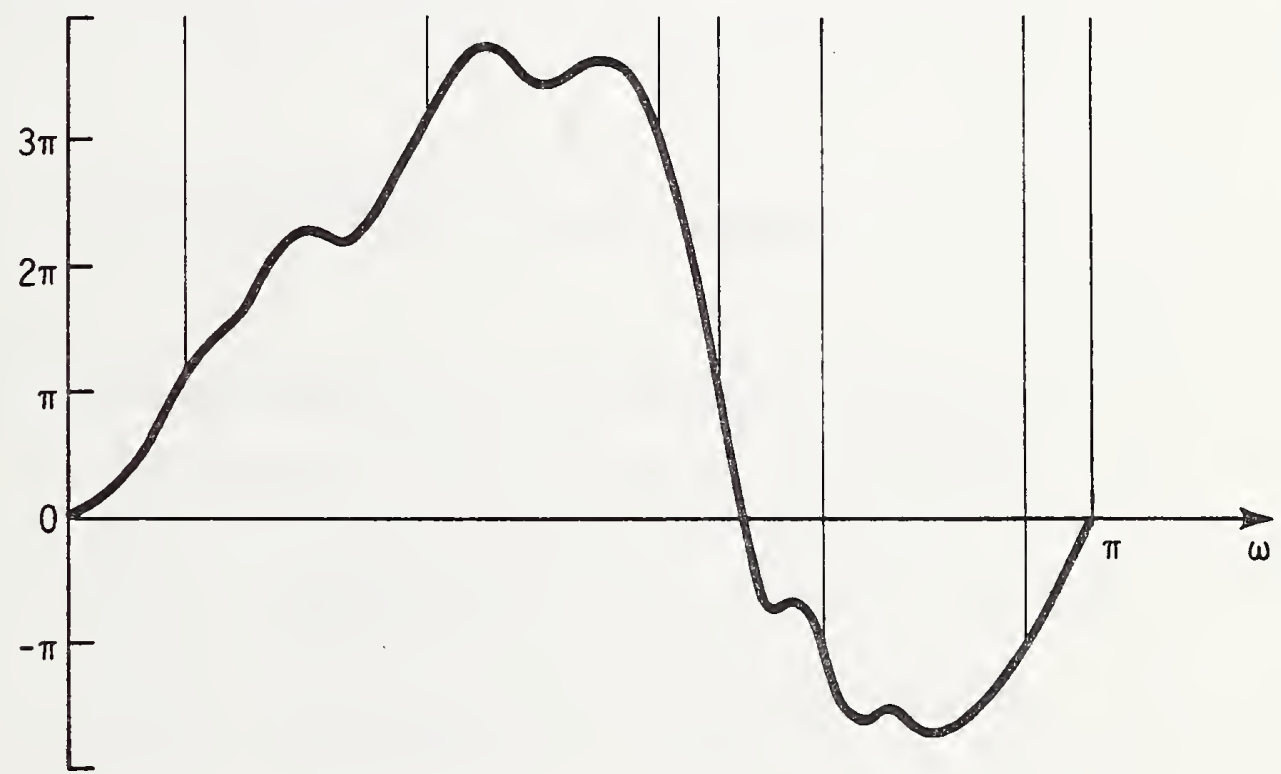

Figure 2.5. The Reconstruction of the Argument Function arg to Create the Continuous carg Function. 


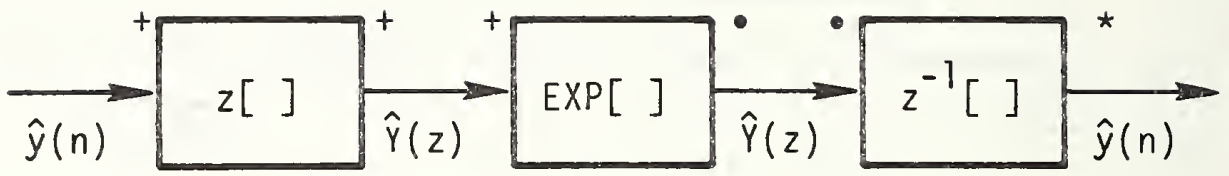

Figure 2.6. The Transform $\mathrm{D}_{\star}^{-1}$. 


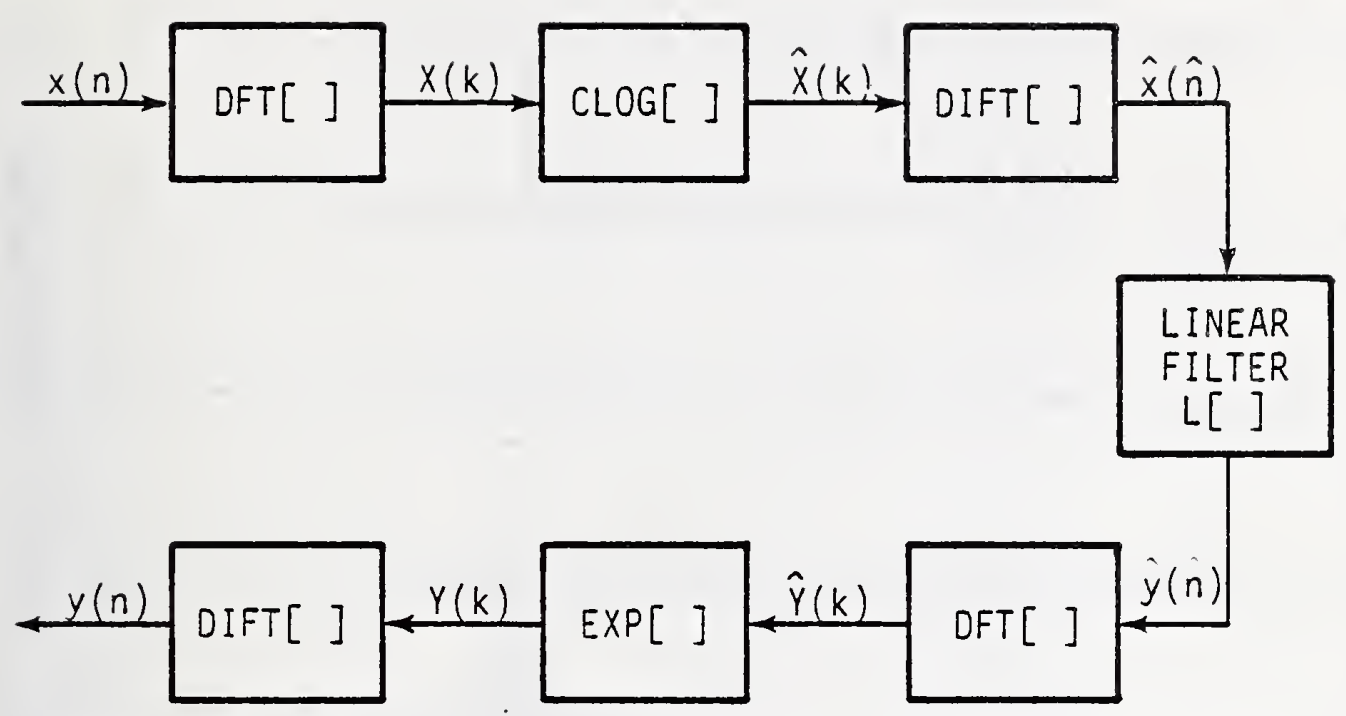

Figure 2.7. The Computation Realization of the Homomorphic Deconvolution Transform. DFT $\equiv$ Discrete Fourier Transform. DIFT $\equiv$ Discrete Inverse Fourier Transform.

CLOG $\equiv$ Continuous Complex Logarithm Function. 


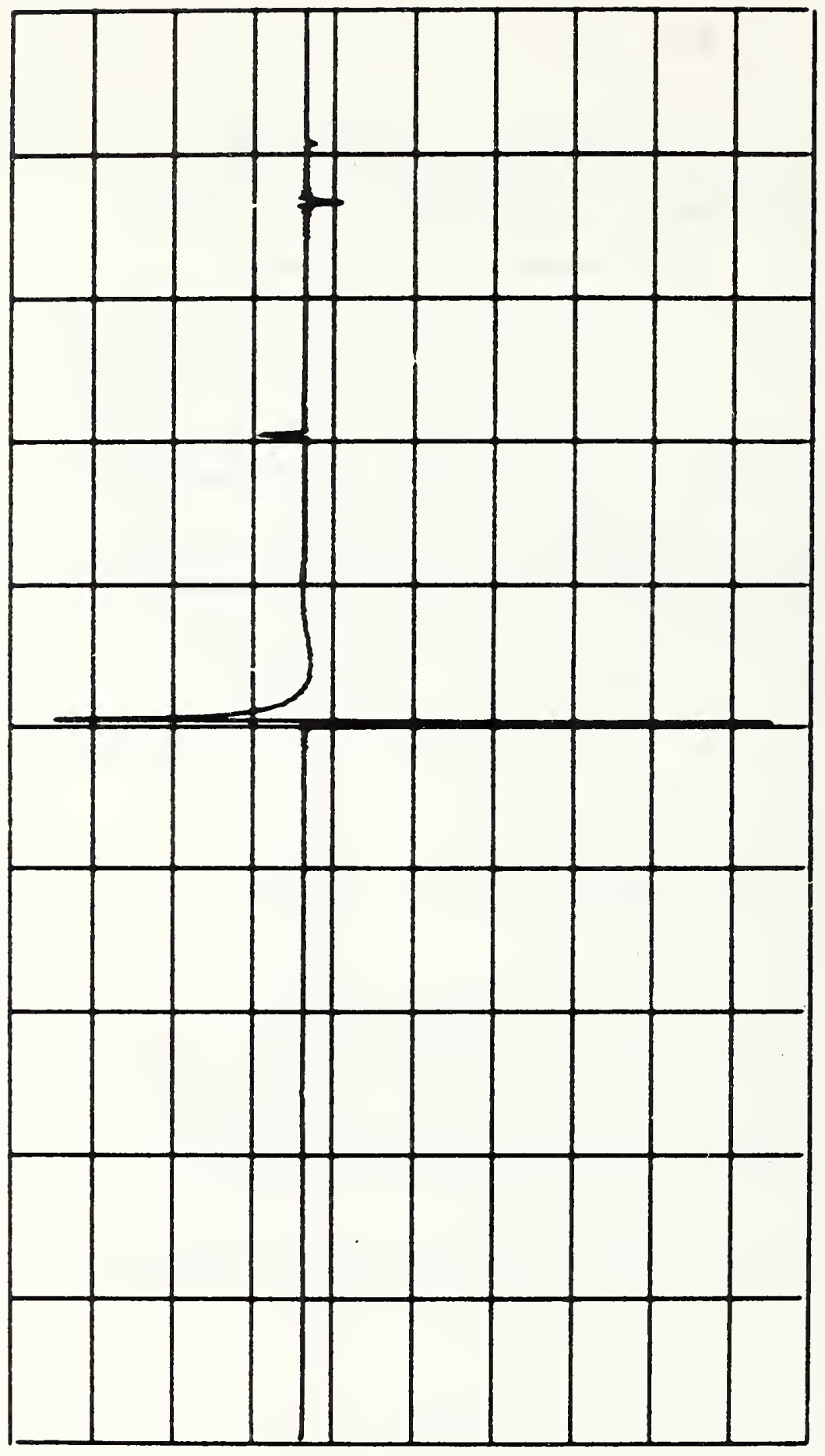

ב 


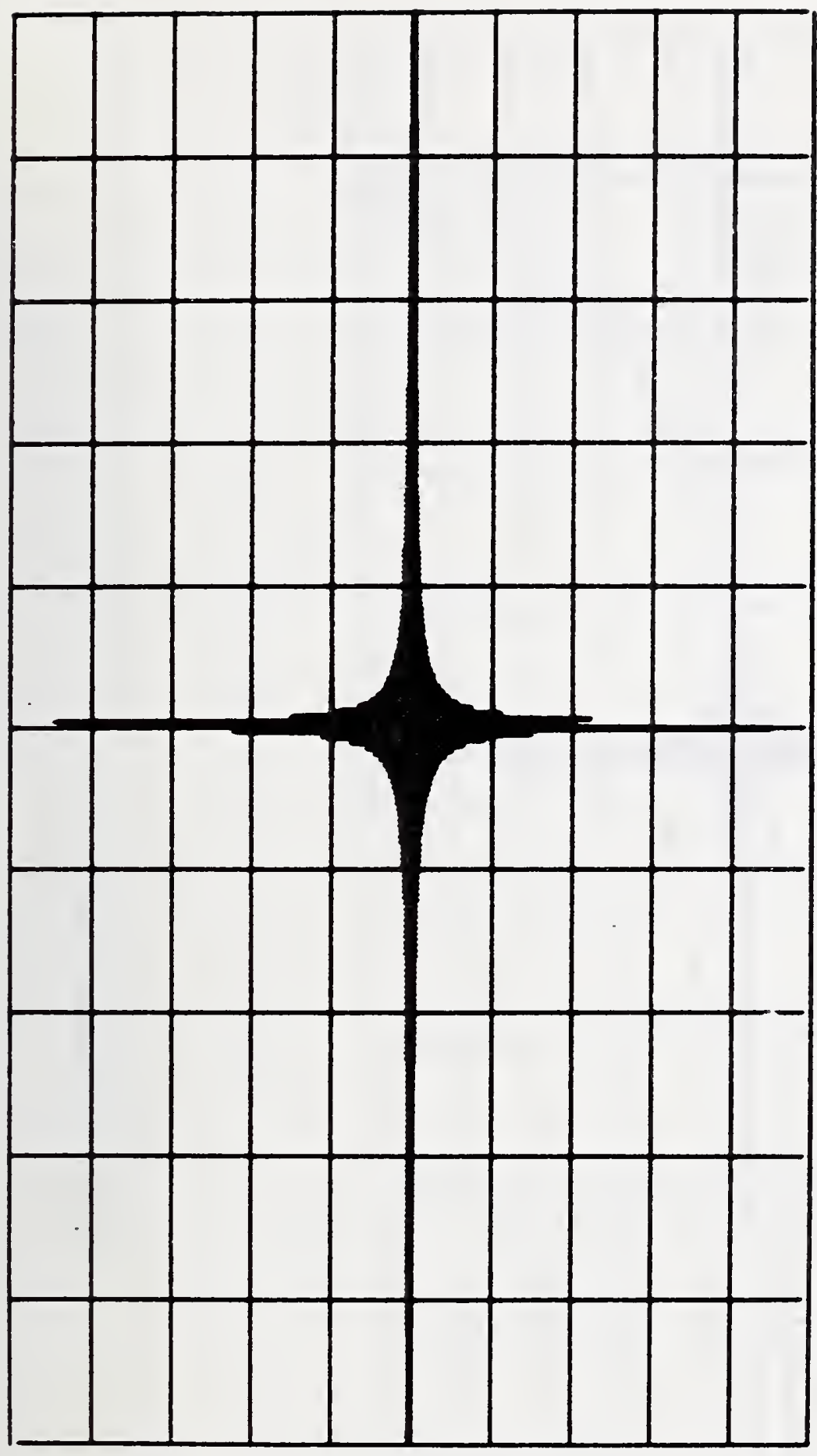

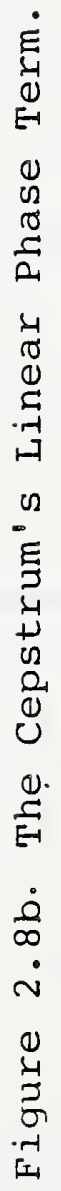




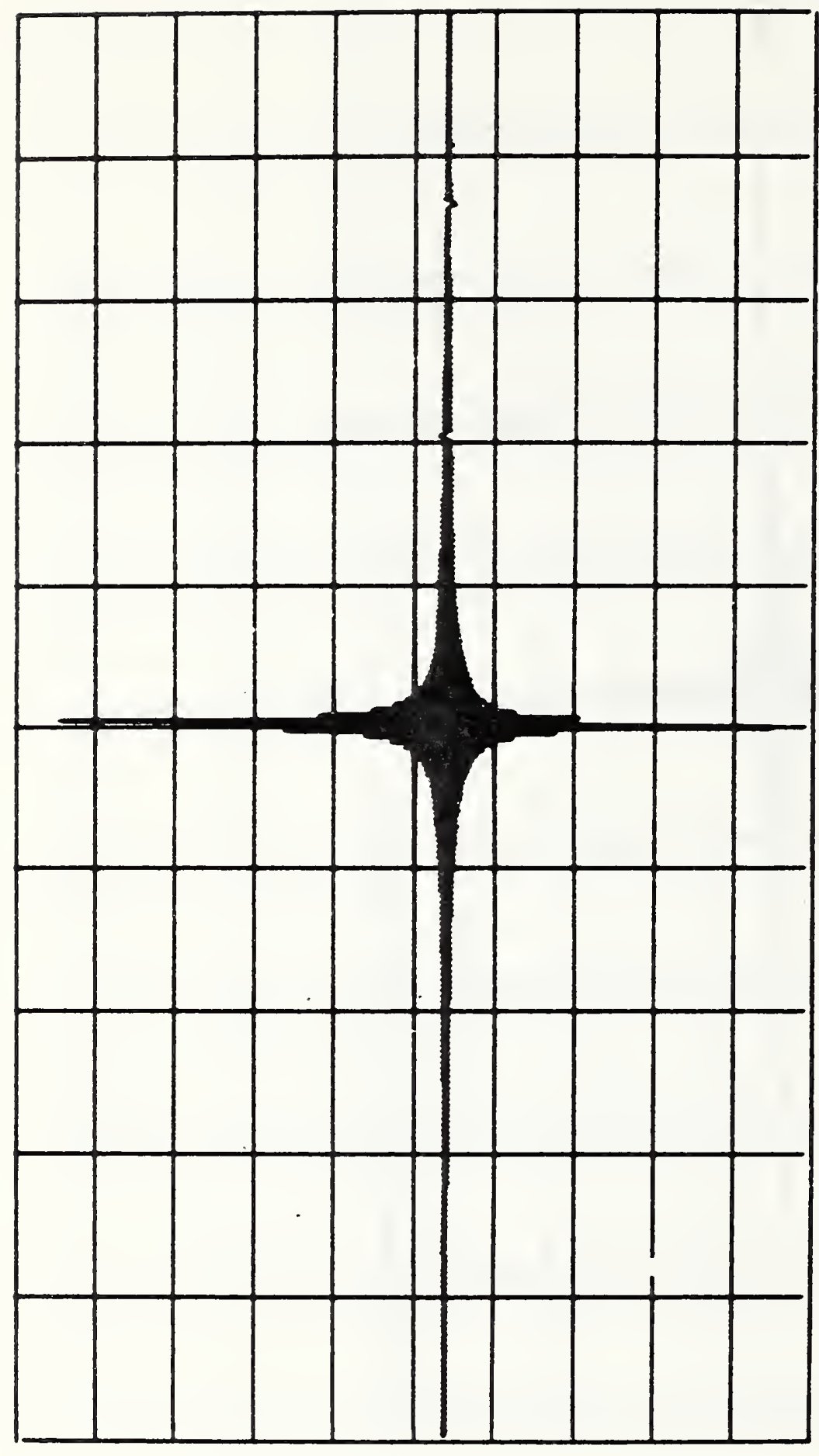

U

•

E

E

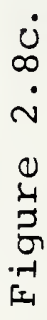

告

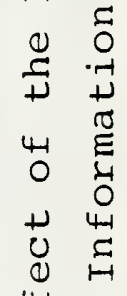

4

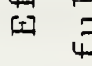

E 0

$\stackrel{\infty}{\sim}$

ปี 


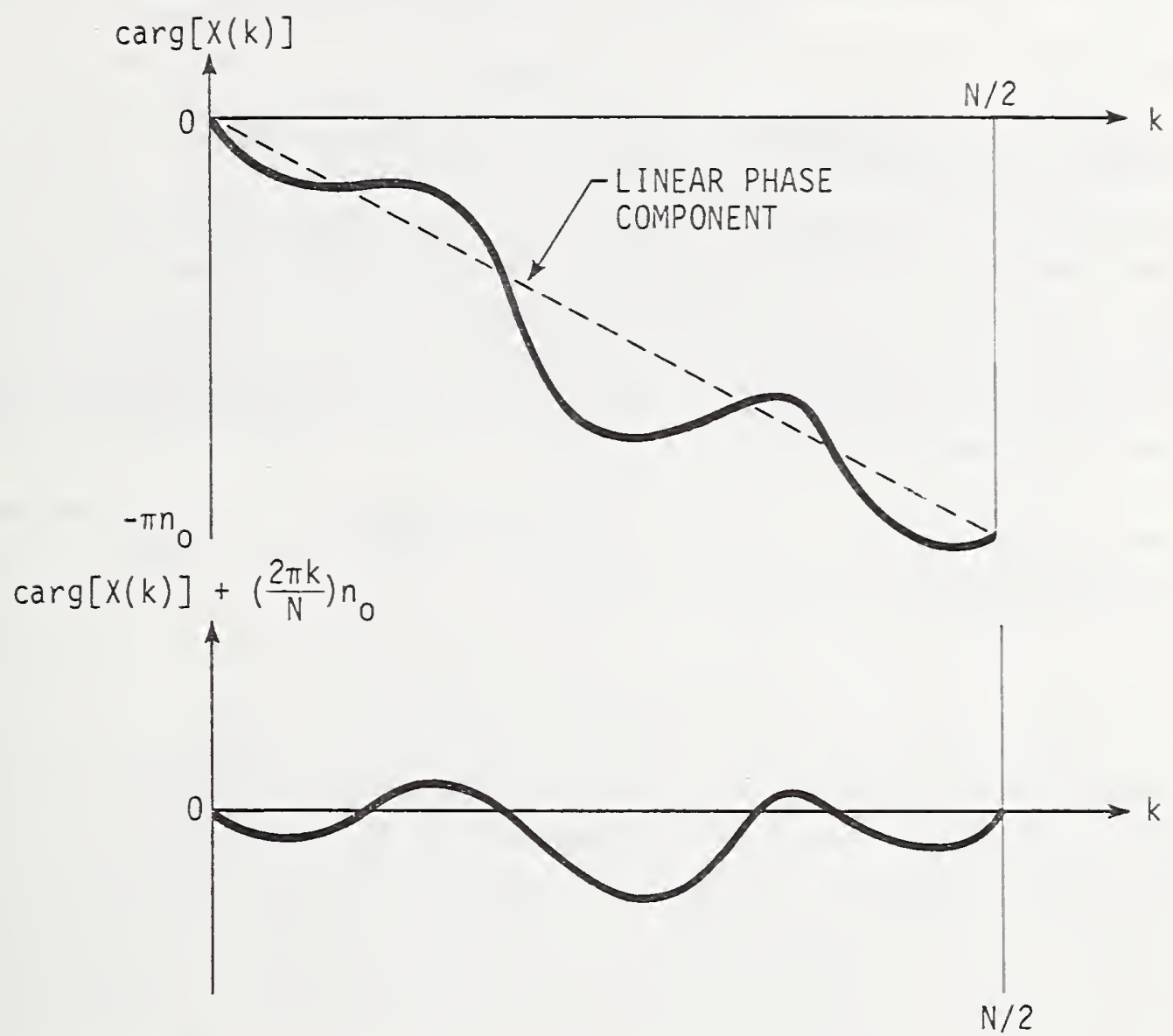

Figure 2.9. The Elimination of the Linear Phase component. 


\subsection{Introduction}

In ehapter 2, the homomorphic deconvolution transform was developed. As it was shown, the separation (deconvolution) idea was based on the property of this transform to convert a convolution of two sequences (waveforms) into a sum of their contributions in a hypothetical time domain called the cepstrum. Separating filters are then to be applied to the cepstrum sequence to select only one contribution at a time. The separation can be realized easily if the two cepstrum contributions were discernible from each other; i.e., each one can be viewed in a separate window, in which case simple (ideal) bandpass type (mathematical) filters can be used for the separation process.

In the present chapter, a class of problems that meet the above mentioned requirement for easy separation will be treated; this class of problems is the analysis of linear microwave networks using time domain techniques.

\subsection{Time Domain Network Analysis}

The time domain method of network analysis is based upon observing the reflections (or transmission) of an incident time domain waveform back from (or through) the network to be analyzed. The observed signal contains information on both the incident signal and the networks impulse response. The typical experimental setups for both the reflection and transmission cases are given in figure 3.1. The method of observing the reflected waveform is commonly called the TDR (time domain reflectometry) method; on the other hand, the abbreviation TDT will be assigned to the time domain transmission measurement method. In TDR, figure 3.1a, the observed reflected waveform can be viewed as the convolution of the incident waveform and the reflection impulse response of the network. While in the TDT, figure $3.1 \mathrm{~b}$, the transmission waveform is the convolution of the incident waveform and the transmission impulse response of the network. The general nature of the impulse responses of the microwave networks that will be considered in this chapter is of the form of a pulse train, because of discretely distributed discontinuities along the network transmission line. The frequency domain form of such impulse responses do have relatively rapid variations (with frequency) compared to the rates of variation occurring in the frequency domain forms of most test (incident) waveforms commonly used in TDR or TDT measurements. Consequently, the application of the homomorphic deconvolution transform will result in clustered groups of components in which the two distinct contributions will be due to the network impulse response and the incident waveform, respectively; and accordingly they will be easily separable. Figure 3.2 shows the homomorphic deconvolution transformation as being applied to a typical time domain waveform that could be encountered in either a TDR or a TDT measurement. 
The microwave networks that will be considered in the next two sections of this chapter will be classified into the following two classes:

(1) Pure resistive networks, i.e., with pure resistive reflections (discontinuities), and this will be considered in section 3.3 .

(2) Networks with reactive discontinuities, which will be dealt with in section 3.4 .

The reason for this classification is, as will be seen later, that a pure resistive network has a train of delta functions as its impulse response in comparison with reactive reflections, where the impulse response contains a train of pulses whose duration and time constants increase with an increase in the reactive parts of the discontinuities. This will appear in the cepstrum domain in the form of more separability for the resistive network as compared to reactive ones.

\subsection{Pure Resistive Networks}

In this section two types of resistive discontinuity problems will be considered: (1) pure resistive loading (discontinuities) along a lossless transmission line, figure 3.3; and (2) the case of commensurate transmission lines, figure 3.4, which is a cascade of lossless transmission lines, each one having different electrical parameters $\left(z_{0}\right.$, $\left.\gamma\right)$. In practice, these two considered cases may include reactive components in their physical realization; but such reactive components are always neglected in the idealized case, and the networks are treated as purely resistive ones. However, the extension to cases with more general reflections including reactive parts will be treated in the next section.

In this section the time domain reflectrometry (TDR) method will be used. The general form of the reflective impulse response $h^{\prime}(t)$ of a microwave resistive network is given by

$$
h^{\prime}(t)=\sum_{n=0}^{\infty} A_{n} \delta\left(t-\tau_{n}\right)
$$

where $t$ is the time variable and both $A_{n}$ and $\tau_{n}$ are constants determined by the network parameters. Assuming an incident waveform $x(t)$ at the input terminals of the network, the reflected waveform $y^{\prime}(t)$ would be the convolution of $x(t)$ and $h^{\prime}(t)$; i.e.,

$$
y^{\prime}(t)=x(t) * h^{\prime}(t)
$$

The observed waveform $y(t)$ at the input gate is the sum of both incident and reflected ones; i.e.,

$$
\begin{aligned}
y(t) & =x(t)+y^{\prime}(t) \\
& =x(t)+x(t) * h^{\prime}(t) \\
& =x(t) *\left(\delta(t)+h^{\prime}(t)\right) \\
& =x(t) * h(t)
\end{aligned}
$$


where

$$
h(t)=\delta(t)+h^{\prime}(t) .
$$

In the frequency domain the signal $h(t)$ transforms into $H\left(e^{j \omega}\right)$ where

$$
\begin{aligned}
H\left(e^{j \omega}\right) & =1+\sum_{n=0}^{\infty} A_{n} e^{-j \omega \tau_{n}} \\
& =1+\sum_{n=0}^{\infty} A_{n} \cos \omega \tau_{n}-j \sum_{n=0}^{\infty} A_{n} \sin \omega \tau_{n}
\end{aligned}
$$

which is clearly seen to be oscillatory in nature (with frequency). On the other hand $\mathrm{X}\left(\mathrm{e}^{j \omega}\right)$ for most physical signals is non-oscillatory; i.e., slowly varying with frequency compared with $\mathrm{H}\left(\mathrm{e}^{j \omega}\right)$. The application of the homomorphic deconvolution can then be expected to separate the two components $x(t)$ and $h(t)$ from their convolution.

In the following the two above described cases of resistive networks will be considered. In each case two examples will be considered and developed; the first of which will be simple (low number of discontinuities) such that the mathematical verification of the results can be followed without too much confusion. The second example containing a large number of discontinuities will be presented in terms of the results of applying the homomorphic deconvolution in order to demonstrate the ability of the method to handle such complicated cases.

In the numerical computations for the examples presented below, 1024 samples in both time and frequency domains were used. The time and frequency sampling intervals were $2.45 \mathrm{ps}$ and $0.4 \mathrm{GHz}$, respectively.

\subsubsection{Case of pure resistive line loading}

The general form of network to be considered in this part is given in figure 3.3. However, as mentioned earlier, only a simple case will be considered as far as the mathematical details are concerned; this is the case of two discontinuities shown in figure 3.5 . Two resistances $R_{1}$ and $R_{2}$ are connected across a lossless transmission line having a characteristic impedance $R_{0}$ at the distances $\ell_{1}$ and $\ell_{2}$ as shown in the figure. Denoting the reflection coefficients at the discontinuities $R_{1}$ and $R_{2}$ as $\rho_{1}$ and $\rho_{2}$, respectively, the reflective impulse response of the network is derived in figure $3.5 \mathrm{~b}$ to be

$$
h^{\prime}(t)=\rho_{1} \delta\left(t-2 \tau_{1}\right)+\rho_{2}\left(1+\rho_{1}\right)^{2} \delta\left(t-\left(2 \tau_{1}+2 \tau_{2}\right)\right)+\ldots
$$

Upon substituting for $\rho_{1}, \rho_{2}, \tau_{1}$, and $\tau_{2}$ by their respective values $-0.2,-0.75,127$ ps, and 84.67 ps that were used in the simulated application, this impulse response becomes

$$
h^{\prime}(t)=-0.2 \delta(t-3 \tau)-0.48 \delta(t-5 \tau)-0.072 \delta(t-7 \tau)-\ldots
$$


where $\tau=84.67$ ps. Figure $3.5 \mathrm{c}$ shows the impulse response $h(t)=\delta(t)+h^{\prime}(t)$ for this specific case.

Figure 3.6 shows the time domain waveform $e_{g}(t)$ of the generator used, while figure 3.7 shows the corresponding frequency domain form. The incident waveform $x(t)$ is given by

$$
x(t)=\frac{e_{g}(t)}{2}
$$

since $R_{0}$ the generator internal resistance is matched to the line resistance. Thus $x(t)$ can also be represented by the figures 3.6 and 3.7 scaled by a factor of one half.

Figures 3.8 through 3.14 show various processing waveforms throughout the steps of the homomorphic deconvolution transform. Figures 3.8 and 3.9 show the observed waveform at the input gate of the network in both time and frequency domains, respectively. Figure 3.10 shows the resultant cepstrum and the linear filters to be used for the separation process. Figures $3.11,3.12,3.13$, and 3.14 give the time and frequency forms of the deconvoluted components $x(t)$ and $h(t)$, respectively. The obtained forms of $x(t)$ and $h(t)$ do coincide with the original form to a great extent. The erroneous oscillations observed in the deconvoluted impulse response is in fact due to sampling errors; i.e., finite number of samples and finite observation time (window).

The figures 3.15 through 3.19 show another example, but a more complicated one with six discontinuities along the line. Figure 3.15 shows the network considered and various values of various network parameters. The generator signal used is the same one as given in figures 3.6 and 3.7. Figure 3.16 shows the convolution $y(t)$, while figure 3.17 shows the corresponding cepstrum. The deconvoluted $x(t)$ and $h(t)$ are given in figures 3.18 and 3.19 , respectively.

\subsubsection{Commensurate transmission lines}

The general form of a commensurate transmission line network was given in figure 3.4. Again, two examples will be considered in this part, one example with two discontinuities and the other one with six discontinuities. The same generator signal that was used in section 3.3.1 will be used in this section.

The two discontinuity example is shown in figure 3.20. Figure 3.20a gives the network; it is a cascade of two lines having the following parameters: 


\section{Line 1}

Characteristic

Impedance

Propagation

Constant

Length

One Way

Travel Time

$$
R_{1}+j 0
$$

$0+j \omega / v_{1}$

$\ell_{1}$

$\tau_{1}=\ell_{1} / \nu_{1}$
Line 2

$$
R_{2}+j 0
$$

$0+j \omega / v_{2}$

$\ell_{2}$

$\tau_{2}=\ell_{2} / \nu_{2}$

The exiting generator has a matching impedance $R_{0}$. Figure $3.20 \mathrm{~b}$ shows the multiple reflections pattern. The reflective impulse response as can be written from this figure is

$$
h^{\prime}(t)=\rho \delta\left(t-2 \tau_{1}\right)-\rho\left(1-\rho^{2}\right) \delta\left(t-\left(2 \tau_{1}+2 \tau_{2}\right)\right)-\rho^{3}\left(1-\rho^{2}\right) \delta\left(t-\left(2 \tau_{1}+4 \tau_{2}\right)\right)-\ldots
$$

While $h(t)=\delta(t)+h^{\prime}(t)$ is shown in figure $3.20 \mathrm{c}$ for the specific case that is to be simulated on the computer, in which

$$
\begin{aligned}
\rho & =0.333, \\
\tau_{1} & =127 \mathrm{ps},
\end{aligned}
$$

and

$$
\tau_{2}=101.6 \mathrm{ps},
$$

this $h(t)$ is given by

$$
h(t)=\delta(t)+0.333 \delta(t-3 \tau)-0.2963 \delta(t-5.4 \tau)-0.0329 \delta(t-7.8 \tau)-\ldots
$$

Figures 3.21 through 3.27 show the processing waveforms throughout the steps of the homomorphic deconvolution transform. Again, figures 3.25 and 3.27 give the deconvoluted $x(t)$ and $h(t)$ which are demonstrated to be in complete agreement with the original forms for $x(t)$ and $h(t)$. On the other hand figures 3.28 through 3.32 show the six commensurate lines case.

\subsection{Networks With Reactive Components}

In the present section two additional applications will be introduced beyond the generalization to more general discontinuities along the line. Section 3.4 .1 will be devoted to the consideration of a TDT type measurement, while section 3.4 .2 will deal with the effects of an unmatched source impedance in a conventional TDR measurement. 


\subsubsection{Time domain transmission (TDT)}

Figure 3.1b, earlier in the chapter, showed the experimental setup for a typical TDI measurement. The example that will be considered in this section is the following:

two impedance discontinuities $z_{1}$ and $Z_{2}$ were connected across a line having characteristic impedance $z_{0}$ and a propagation constant $\gamma_{0}$ at distances $d_{1}$ and $d_{2}$ as shown in figure 3.33 . A generator of matched impedance excites the network; $e_{g}(t)$ is the generator time domain waveform. The aim of this specific problem was to see how the TDT waveforms would yield to the homomorphic deconvolution transform.

In the numerical computations for the present example, 1024 samples in both time and frequency domains were used. The time and frequency sampling intervals were $4.9 \mathrm{ps}$ and $0.2 \mathrm{GHz}$, respectively.

The values of the network parameters that were used in this specific example were as follows:

line: $7 \mathrm{~mm}$ precision coaxial line (50 ת) [6]

$$
\begin{aligned}
& \mathrm{z}_{1}: \text { Capacitive, } \mathrm{c}_{1}=0.3 \mathrm{pf} \\
& \mathrm{z}_{2}: \text { Capacitive, } c_{2}=0.1 \mathrm{pf} \\
& \mathrm{d}_{2}: 3.54 \mathrm{~cm}
\end{aligned}
$$

The waveform of the generator $e_{g}(t)$ is given in figure 3.34. The network was simulated on a digital computer, and the resultant TDT waveform at the output terminals $e_{0}(t)$ is given in figure 3.35. The frequency domain form $E_{0}\left(e^{j \omega}\right)$ of $e_{0}(t)$ is given by the relation

$$
E_{0}\left(e^{j \omega}\right)=\frac{E_{g}\left(e^{j \omega}\right)}{2} \frac{\exp \left[-\gamma_{0}(\omega) \cdot\left(d_{1}+d_{2}\right)\right]\left[1+\rho_{1}(\omega)\right]\left[1+\rho_{2}(\omega)\right]}{\left[1-\rho_{1}(\omega) \rho_{2}(\omega) \exp \left(-2 \gamma_{0}(\omega) d_{2}\right)\right]}
$$

where $E_{g}\left(e^{j \omega}\right)$ is the frequency domain form of $e_{g}(t)$, and $p_{1}(\omega)$ and $\rho_{2}(\omega)$ are the reflection coefficients corresponding to the impedance discontinuities $Z_{1}(\omega)$ and $Z_{2}(\omega)$, respectively. The magnitude of $E_{0}\left(e^{j \omega}\right)$ is given in figure 3.36. The homomorphic deconvolution transform, figure 3.2 , is to be applied on the output signal to separate its convolution components. As was mentioned before, the fiitration in the cepstrum domain separates two components that have substantially different rates of variation in their frequency domain forms. By examining eq. ( 3.11 ) it can be concluded that the slowly varying component $E_{\text {os }}\left(e^{j \omega}\right.$ ) would be

$$
E_{o s}\left(e^{j \omega}\right)=\frac{E_{g}\left(e^{j \omega}\right)}{2} \exp \left[-\gamma_{0}(\omega) \cdot\left(d_{1}+d_{2}\right)\right] \quad\left[1+p_{1}(\omega)\right]\left[1+\rho_{2}(\omega)\right]
$$

which is the first path signal through the network, while the rapidly varying one $E_{\text {or }}\left(e^{j \omega}\right)$ is the multiple reflection effect,

$$
E_{o r}\left(e^{j \omega}\right)=\frac{1}{\left[1-o_{1}(\omega) \rho_{2}(\omega)\right] \exp -2 \gamma_{0}(\omega) d_{2}} .
$$


These eqs. (3.12) and (3.13) can be used to determine up to two unknown system parameters, provided that all other network parameters are known. To show this, eq. (3.13) will be used to calculate $\rho_{2}(\omega)$ assuming that $\rho_{1}\left(e^{j \omega}\right)$ is known; the values of $\rho_{2}(\omega)$ are to be used to calculate the corresponding impedance $\mathrm{z}_{2}(\omega)$.

The above mentioned steps, in the current section, were followed, and the results are shown in figures 3.37 through 3.42. Figure 3.37 gives the cepstrum of $e_{0}(r)$. Figures 3.38 through 3.41 give the slowly and rapidly varying components in both frequency and time domain forms, respectively. The resultant values for $E_{\text {or }}\left(e^{j \omega}\right)$ were used for the calculation of $\mathrm{z}_{2}(\omega)$ as indicated earlier. The computed values for $\mathrm{z}_{2}(\omega)$ showed a pure capacitive impedance, and the capacitance $\mathrm{C}_{2}$ is plotted in figure 3.42. The figure shows erroneous oscillation about the actual given value. These error oscillations are again due to sampling errors. Part of the errors are also due to the imperfect separation in the cepstrum domain because of the extended long tails due to reactive reflections on the line.

\subsubsection{Time domain reflections (TDR) with reactive loading/unmatched generator impedance [7,8]}

In this case the TDR measuring technique will be presented in its conventional form, showing the limitations of the method. Then it will be shown how the homomorphic deconvolution transform helps in offsetting these limitations.

\subsubsection{TDR measurements}

Figure 3.43 shows the typical TDR experimental setup for measuring the reflection coefficient of the line termination $\rho_{L}(\omega)$. The general procedure is to record the displayed signal for the termination under test and two other reference terminations, usually a short circuit and a $z_{o}(\omega)$ termination.* The complex frequency domain forms of the three recorded signals are given by

$$
\begin{aligned}
& E_{d M}\left(e^{j \omega}\right)=E_{g}\left(e^{j \omega}\right) \cdot \frac{Z_{o}(\omega)}{Z_{o}(\omega)+z_{g}(\omega)} \cdot T(\omega) \\
& E_{d S}\left(e^{j \omega}\right)=E_{g}\left(e^{j \omega}\right) \cdot \frac{Z_{o}(\omega)}{Z_{o}(\omega)+z_{g}(\omega)} \cdot \frac{1-\exp (-2 \gamma(\omega) l)}{1+\rho_{g}(\omega) \cdot \exp (-2 \gamma(\omega) \ell)} \cdot T(\omega) \\
& E_{d L}\left(e^{j \omega}\right)=E_{g}\left(e^{j \omega}\right) \cdot \frac{Z_{o}(\omega)}{Z_{o}(\omega)+z_{g}(\omega)} \cdot \frac{1+\rho_{L}(\omega) \exp (-2 \gamma(\omega) \ell)}{1-\rho_{L}(\omega) \rho_{g}(\omega) \exp (-2 \gamma(\omega) l)} \cdot T(\omega)
\end{aligned}
$$

corresponding to $Z_{0}(\omega)$, short circuit, and $Z_{L}(\omega)$ terminations, respectively. One cannot solve the above three equations, as they are, to obtain $\rho_{L}(\omega)$.

*For an exciting waveform which becomes of absolutely constant value before the reflections arrive, the $\mathrm{z}_{\mathrm{o}}$ measurement is not necessary. 


\subsubsection{Conventional =ethod}

The conventional method for evaluating $\rho_{I}(\omega)$ is to consider only the TDR signals occurring in the ine window $0<t<4 T$, where $I$ is the travel rime on the line of length i. In this case ecss. (3.11), (3.12), and (3.13) will take the sitplified foras

$$
\begin{aligned}
& E_{\mathrm{dY}, 4 T}\left(e^{j \omega}\right)=E_{g}\left(e^{j \omega}\right) \cdot \frac{Z_{0}(\omega)}{Z_{0}(\omega)+Z_{g}(\omega)} \cdot I(\omega) \\
& E_{c S, 4 T}\left(e^{j(-)}\right)=E_{g}\left(e^{j \omega}\right) \cdot \frac{Z_{o}(\omega)}{Z_{0}(\omega)+Z_{g}(\omega)} \cdot I(\omega) \cdot\left[1-(1+j g(\omega)) \exp \left(-2 \gamma(\omega) s_{0}\right)\right] \\
& E_{d I, 4 T}\left(e^{j \omega}\right)=E_{g}\left(e^{j \omega}\right) \cdot \frac{Z_{o}(\omega)}{Z_{0}(\omega)+Z_{g}(\omega)} \cdot I(\omega) \cdot\left[1+\rho_{L}(\omega)\left(1+\rho_{g}(\omega)\right) \exp (-2 \gamma(\omega) \ell)\right]
\end{aligned}
$$

frou wich $o_{I}(\omega)$ can be determined;

$$
\rho_{L}(\omega)=-\frac{E_{d I, L I}(\omega)-E_{C M, L T}(\omega)}{E_{d S, L I}(\omega)-E_{d Y, L I}^{(\omega)}} .
$$

This method works only if the second and subsequent reflections do not overlap the first one. Figure 3.44 shows wo cases of IDR signals: the non-overlapping reflections where the conventional method works, and the overlapping realections in which the conventional Eethod Eails.

\subsubsection{The application of the hotonorphic deconvolution nechod}

In the homo=orphic deconvolution method, see figure 3.2, the complete forms, eqs. (3.11), (3.12), and (3.13), are used to obtain not only $\rho_{L}(\omega)$ but also $\rho_{g}(\omega), r(\omega)$, and the product $E_{g}\left(e^{j \omega}\right) I(\omega)$. The process is begun by first obtaining the differences of eq. (3.15) - eq. (3.14) and eq. (3.16) - eq. (3.14) which are

$$
\begin{aligned}
E_{S}\left(e^{j \omega}\right)= & E_{d S}\left(e^{j-j}\right)-E_{d U d}\left(e^{j \omega}\right) \\
= & E_{g}\left(e^{j \omega}\right) \cdot \frac{Z_{0}(\omega)}{Z_{0}(\omega)+Z_{g}(\omega)} \cdot T(\omega) \cdot \exp (-2 \gamma(\omega) i) \\
& (-1) \cdot\left(\frac{1+\rho_{g}(\omega)}{1+\sigma_{g}(\omega) \exp (-2 \gamma(\omega) 2)}\right)
\end{aligned}
$$


$E_{L}\left(e^{j \omega}\right)=E_{d L}\left(e^{j \omega}\right)-E_{d M}\left(e^{j \omega}\right)$

$$
\begin{gathered}
=E_{g}\left(e^{j \omega}\right) \cdot \frac{z_{0}(\omega)}{z_{o}(\omega)+z_{g}(\omega)} \cdot T(\omega) \cdot \exp (-2 \gamma(\omega) l) \\
\cdot \rho_{L}(\omega) \cdot\left(\frac{1+\rho_{g}(\omega)}{1-\rho_{g}(\omega) \rho_{L}(\omega) \exp (-2 \gamma(\omega) \ell)}\right) .
\end{gathered}
$$

Using the homomorphic deconvolution technique, each of the two equations, eqs. (3.21) and (3.22), can be split into two components, the slowly varying component and the rapidly varying one (subscripted by $s$ and $r$ in the following equations, respectively).

$$
\begin{aligned}
& E_{S S}\left(e^{j \omega}\right)=E_{g}\left(e^{j \omega}\right) \cdot \frac{z_{o}(\omega)}{Z_{o}(\omega)+z_{g}(\omega)} \cdot T(\omega) \cdot \exp (-2 \gamma(\omega) l) \cdot(-1) \\
& E_{S r}\left(e^{j \omega}\right)=\frac{1+\rho_{g}(\omega)}{1+\rho_{g}(\omega) \cdot \exp (-2 \gamma(\omega) l)} \\
& E_{L s}\left(e^{j \omega}\right)=E_{g}\left(e^{j \omega}\right) \cdot \frac{z_{o}(\omega)}{Z_{o}(\omega)+z_{g}(\omega)} \cdot T(\omega) \cdot \exp (-2 \gamma(\omega) l) \cdot \rho_{L}(\omega) \\
& E_{L r}\left(e^{j \omega}\right)=\frac{1+\rho_{g}(\omega)}{1-\rho_{g}(\omega) \cdot \rho_{L}(\omega) \cdot \exp (-2 \gamma(\omega) l)} \cdot
\end{aligned}
$$

The above four equations together with eq. (3.14) can be used to solve for $\rho_{L}(\omega), \rho_{g}(\omega)$, $\gamma(\omega)$, and the product $E_{g}\left(e^{j \omega}\right) T(\omega)$.

The homomorphic deconvolution technique applied to TDR problems not only gives access to more of the microwave network parameters but also works for overlapping reflection cases, where the conventional methods fails.

\subsubsection{Application}

A computer program was written to simulate the TDR network of figure $3.43 \mathrm{~b}$ with parallel $\mathrm{R}-\mathrm{C}$ combinations to serve as reflective load and generator impedances. The homomorphic deconvolution technique was applied to the waveforms of the simulated model; the network parameters and various waveforms are given in figures 3.45 through 3.50 .

In the numerical computations for the present example 1024 samples were used. The time and frequency sampling intervals were 2.45 ps and $0.4 \mathrm{GHz}$, respectively.

Equations (3.23) and (3.25) were used to calculate the reflection coefficient $\rho_{L}(\omega)$ from the transform domain data of the separated signals (shown in the time domain, figures 3.48 and 3.50). Then the resultant $\rho_{L}(\omega)$ was used to determine $R$ and $C$ of the load impedance 
$\mathrm{Z}_{\mathrm{L}}(\omega)$. The calculated values of $\mathrm{R}$ and $\mathrm{C}$ vs. frequency, figures 3.51 and 3.52 , oscillate around their respective actual values. These erroneous oscillations which correspond to those indicated in figures 3.48 and 3.50 are again due to (a) the finite number of samples considered, (b) the finite observation time window, and (c) the imperfect separation in the cepstrum due to overlapping long tails of the different components.

\subsection{Summary and Conclusions}

The present chapter presented several classes of time domain measurement problems in the field of linear microwave network analysis. The homomorphic deconvolution transform, that was developed in chapter 2, was used in deconvoluting various convolutions in these problems. The examples that were considered are as follows:

(1) Pure resistive loading along a transmission line.

(2) Commensurate transmission line networks.

(3) TDT type measurement with two reactive discontinuities.

(4) Conventional TDR measurement to determine a reactive load impedance.

The homomorphic deconvolution method was proven to be very effective in separating convolutions. The method was not limited by the overlap of consequent reflections in the time domain. 


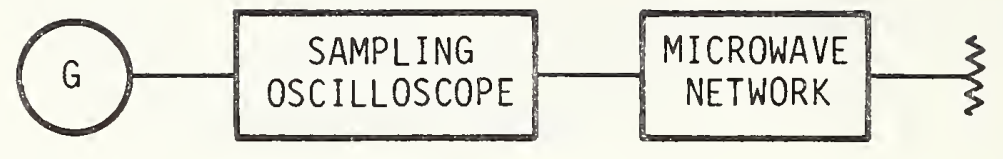

a. TDR set Up.

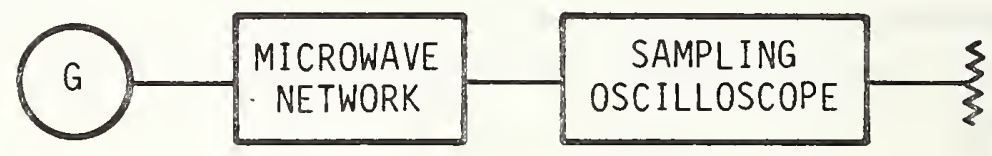

b. TDT set Up.

Figure 3.1. Time Domain Measurements. 


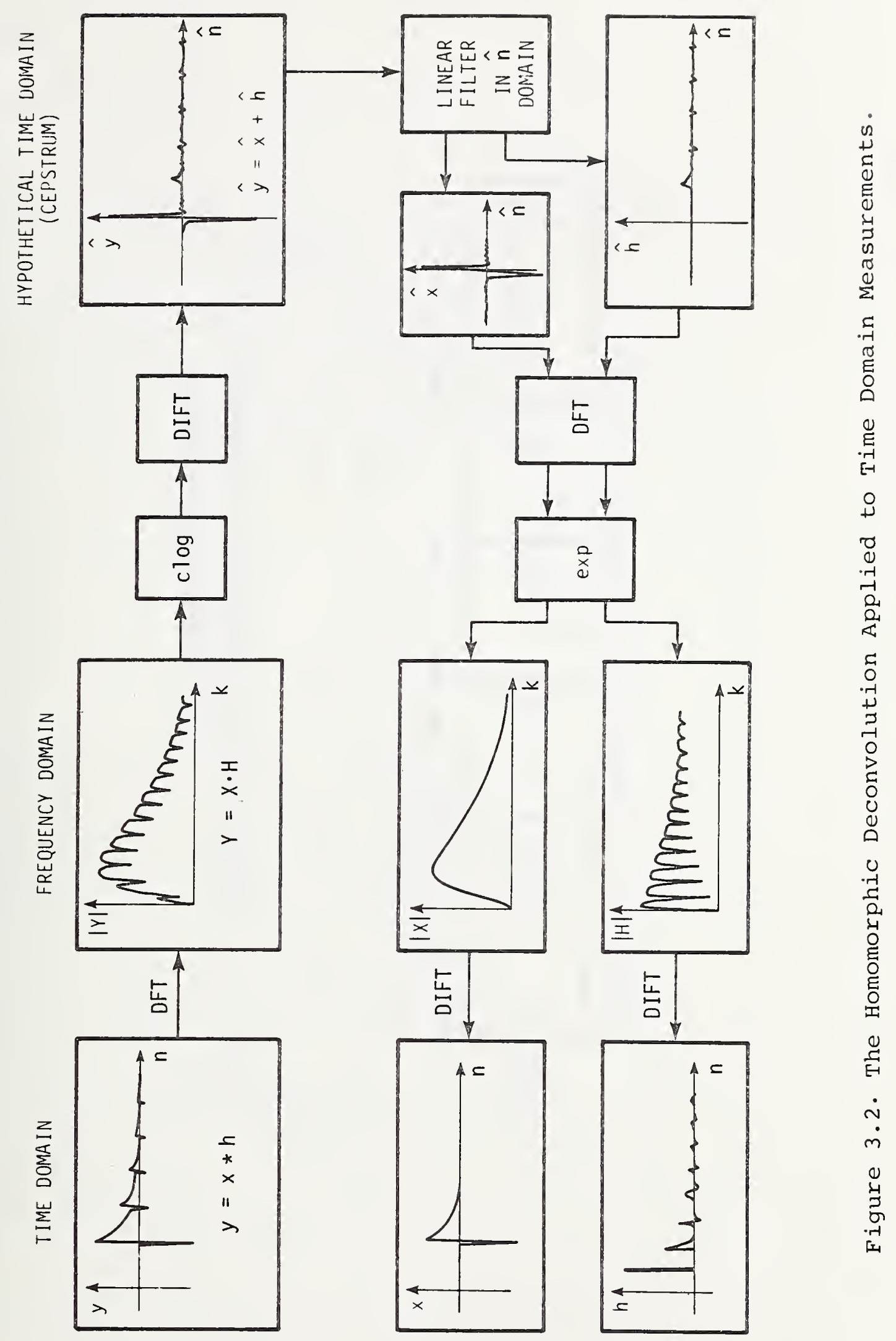




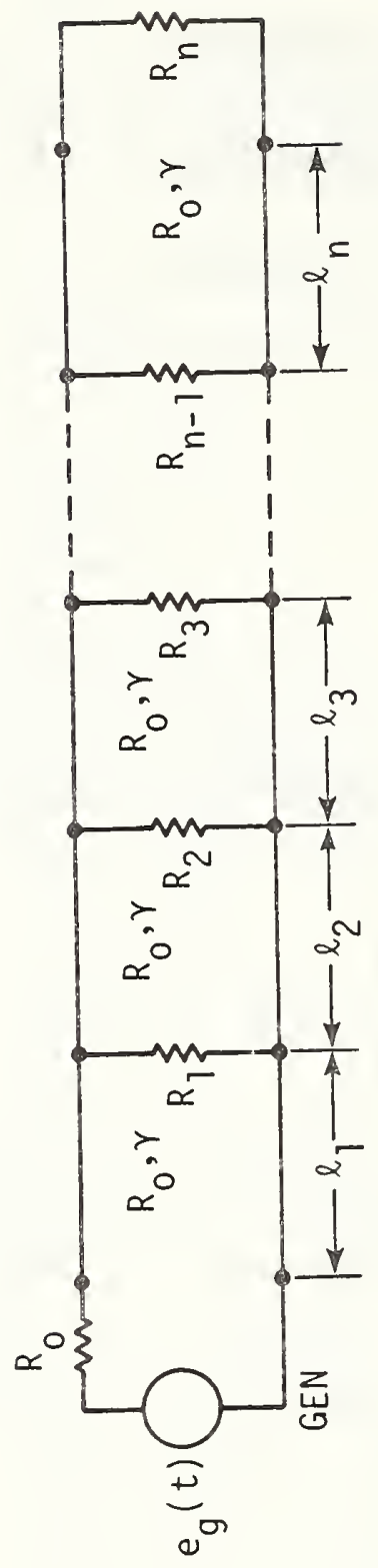

$\begin{array}{cc}0 & 0 \\ 0 & 0 \\ -1 & -1 \\ 0 & -1 \\ 0 & -1 \\ 0 & 1 \\ 0 & 0 \\ 1 & 0 \\ 0 & 0\end{array}$

in

U)

- $C$

O)

(1) त

岁

.

$\dot{m}$

(1)

รู

[4 


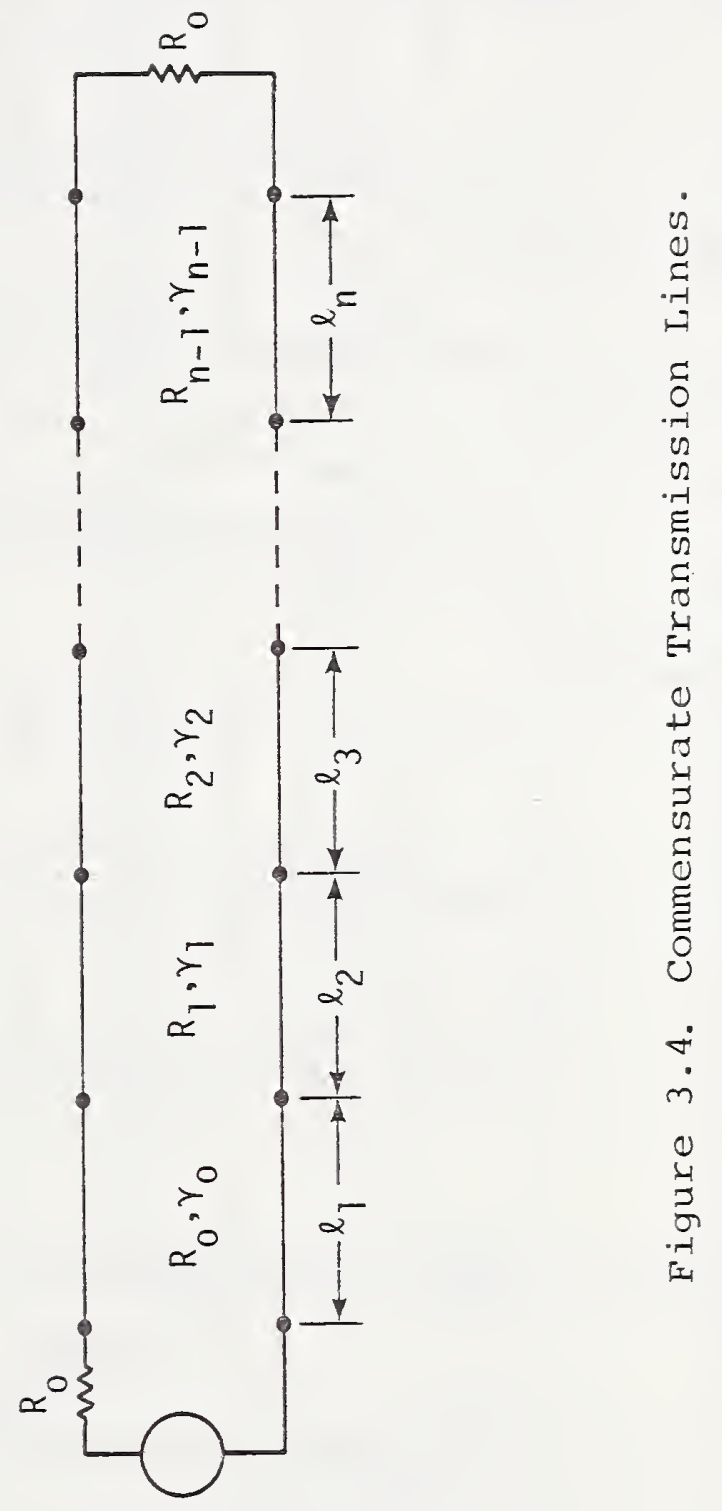

$-33-$ 
a. NETWORK

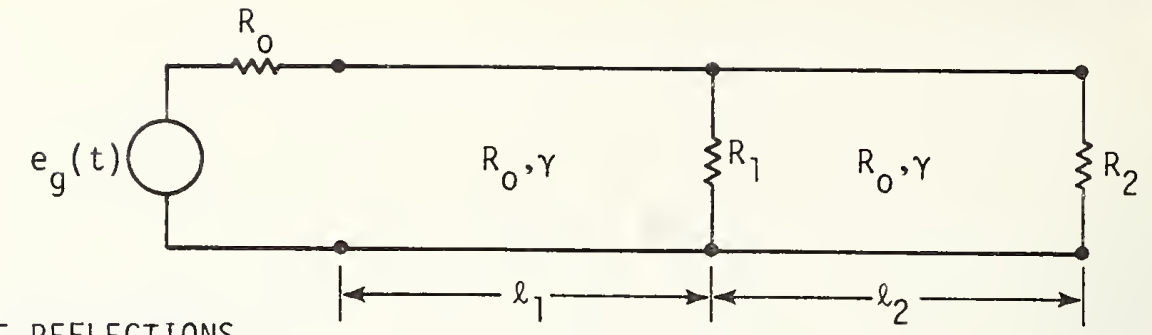

b. MULTIPLE REFLECTIONS

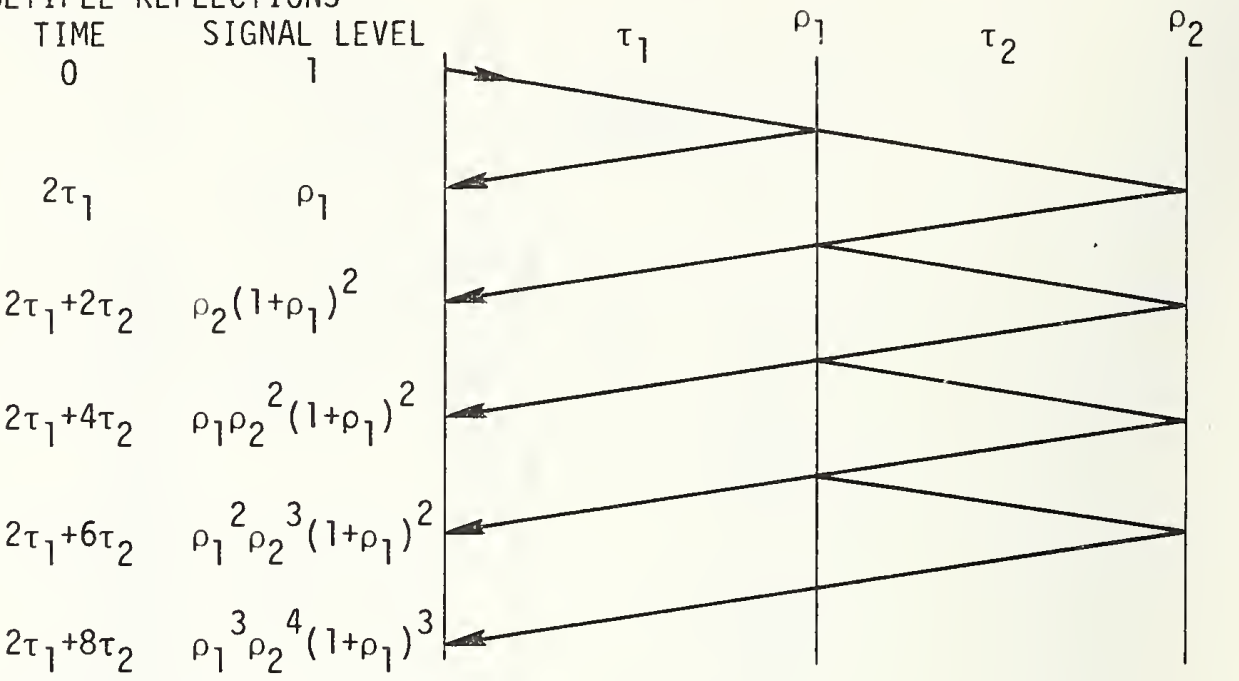

c. IMPULSE RESPONSE

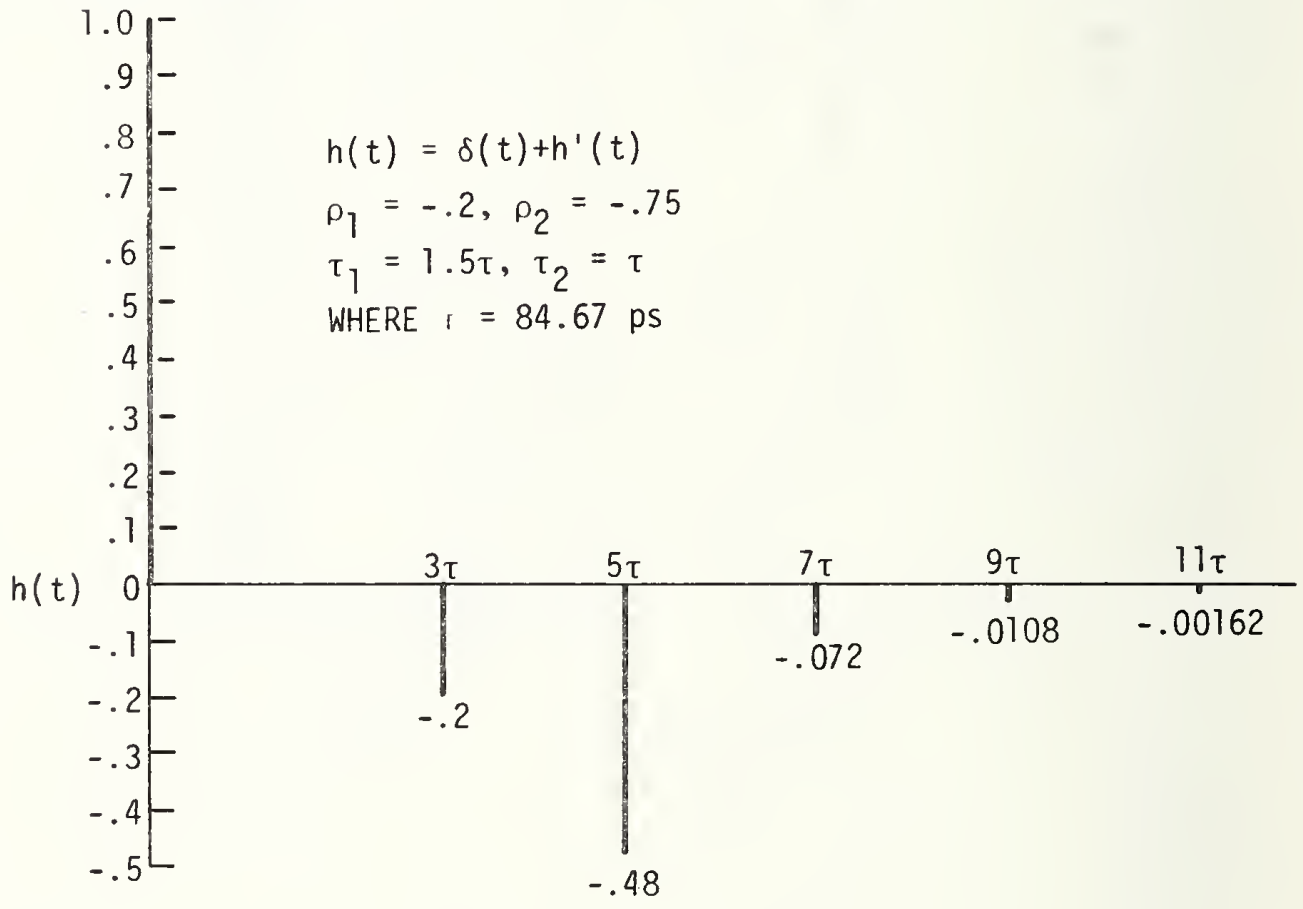

Figure 3.5. Case of Two Reflections. 


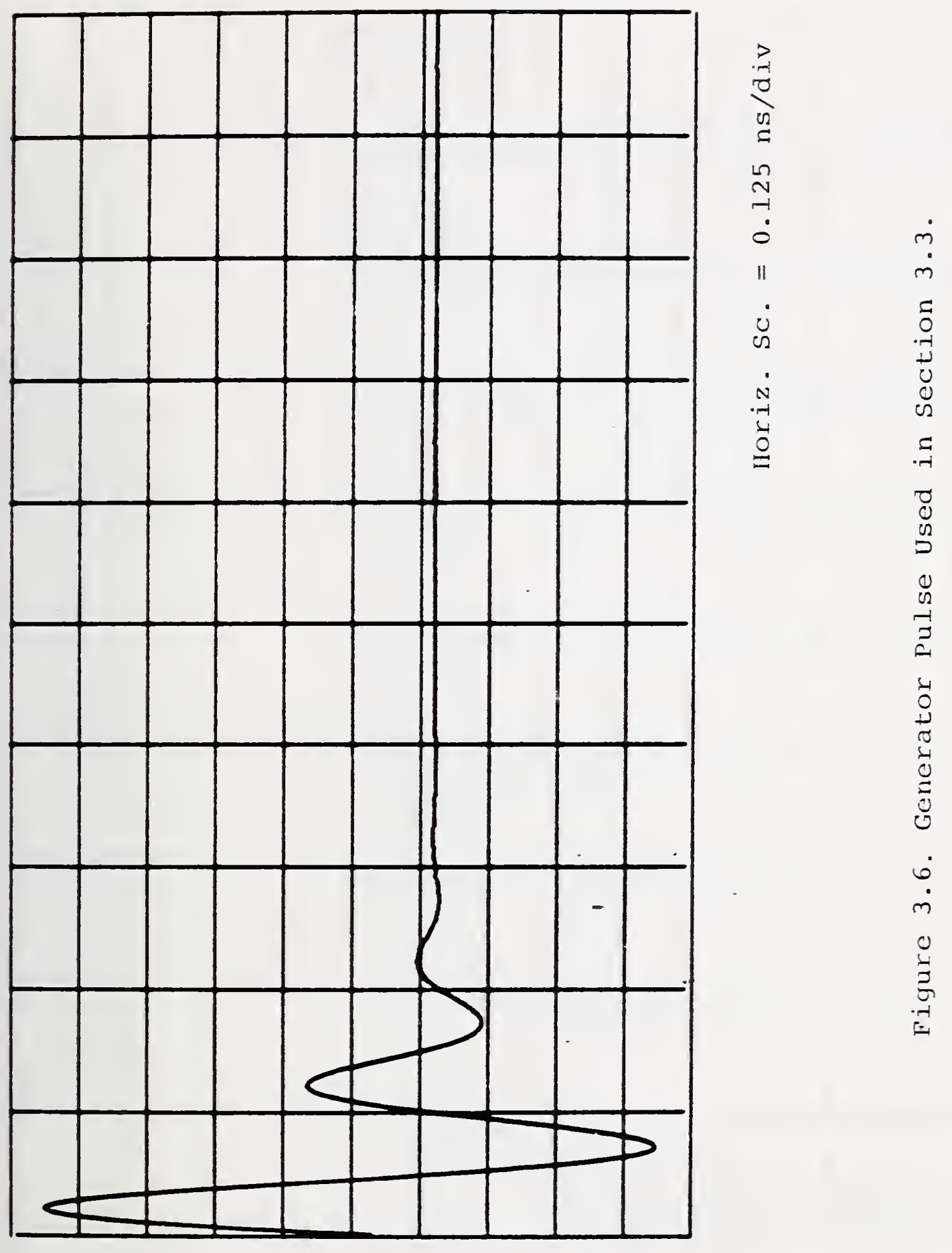




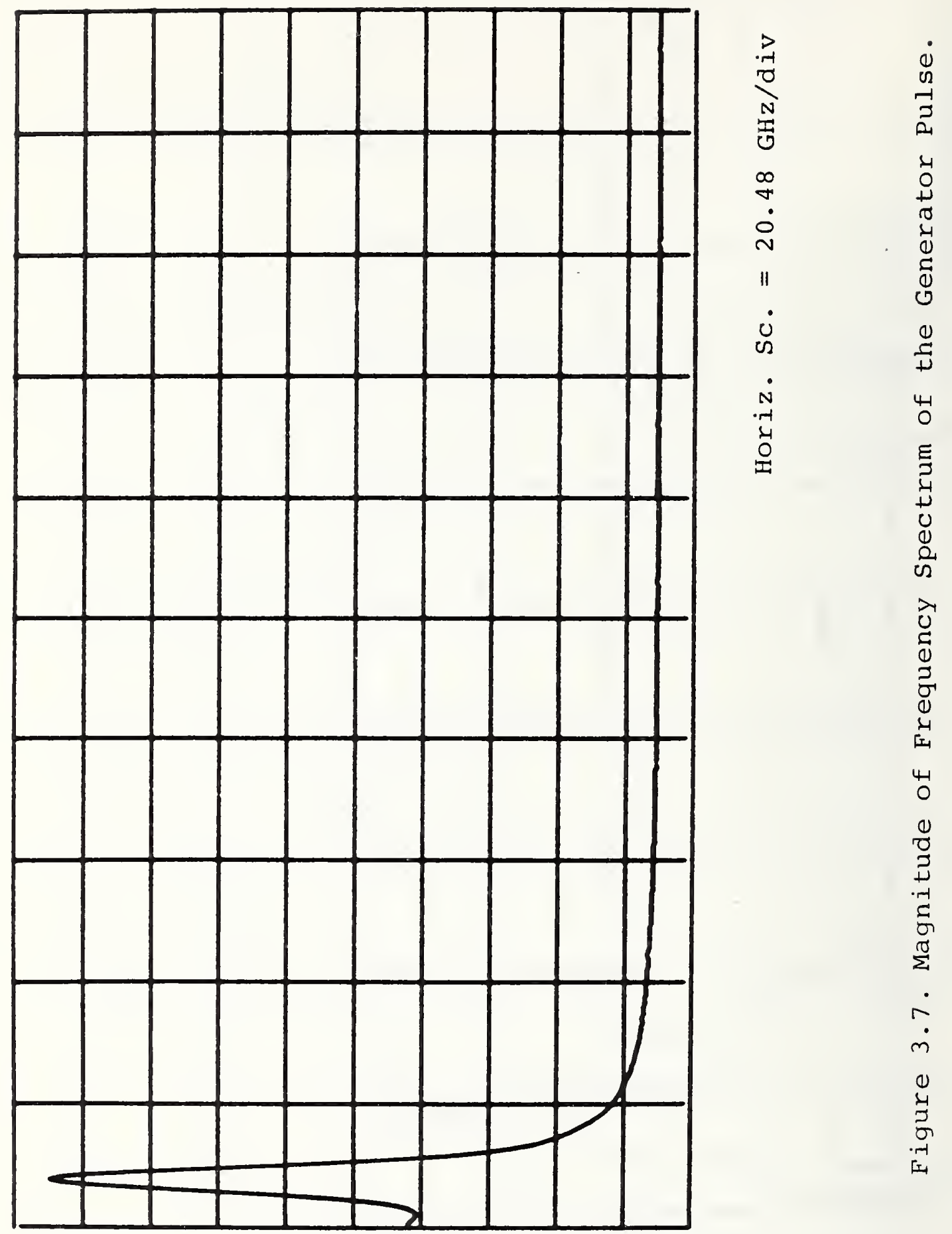




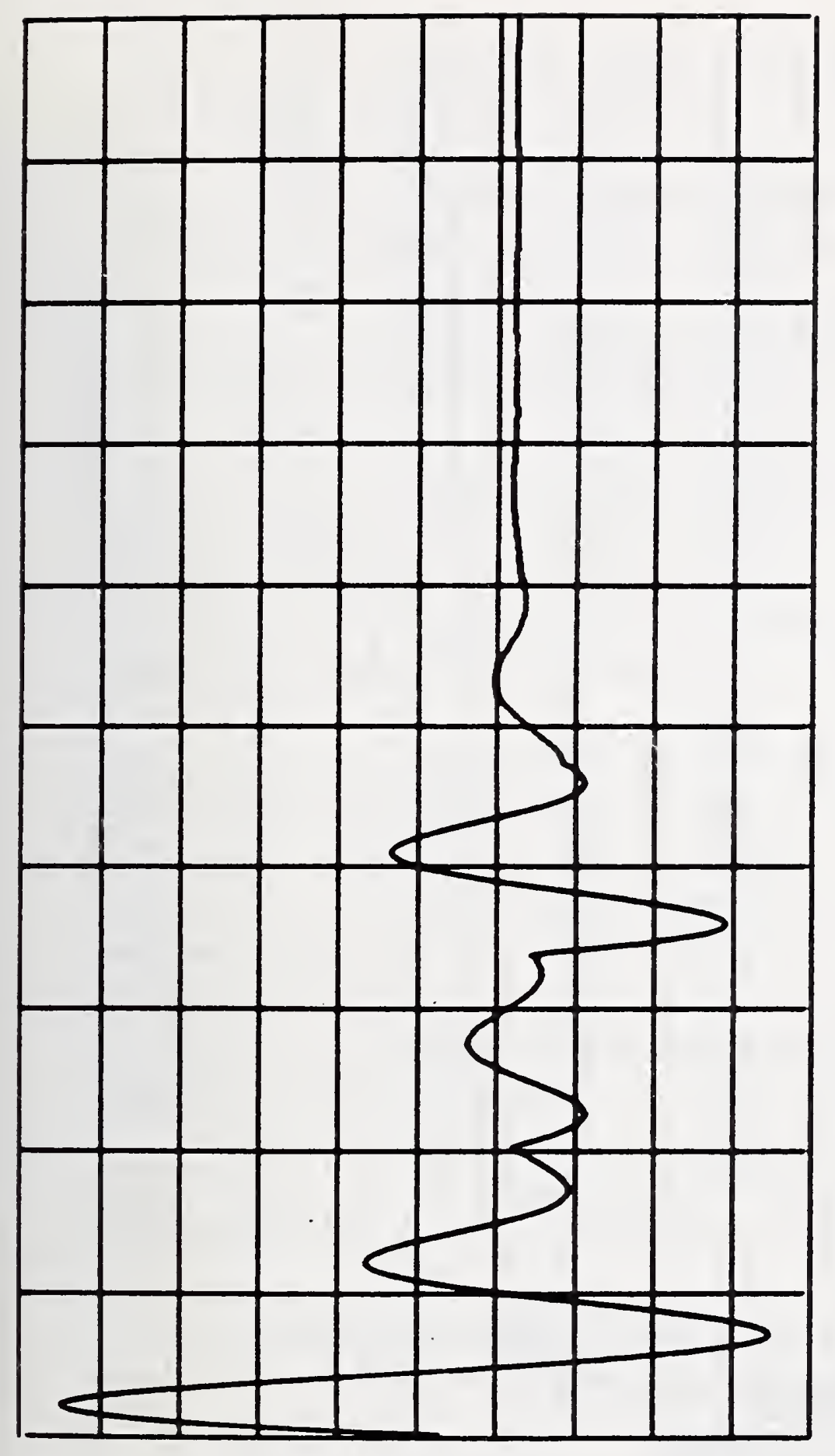

0ี

点

ก 0

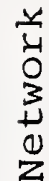

$\stackrel{0}{+1}$

4

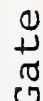

تِّم

$+$

$E$
4
0
4
0
3
0
3
0
0
0
0
0
02
0
0

$\infty$

$\dot{m}$

鳬 

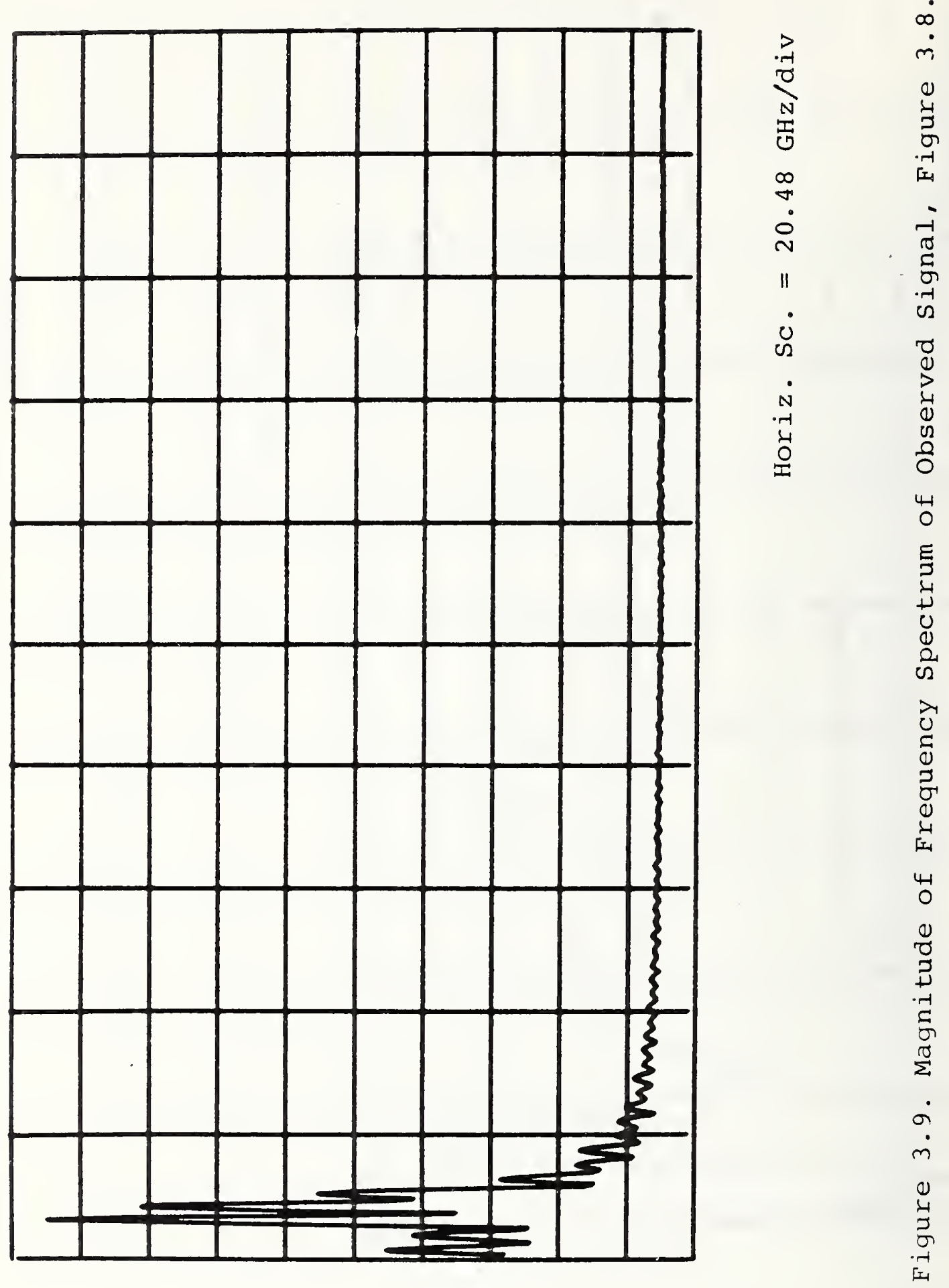


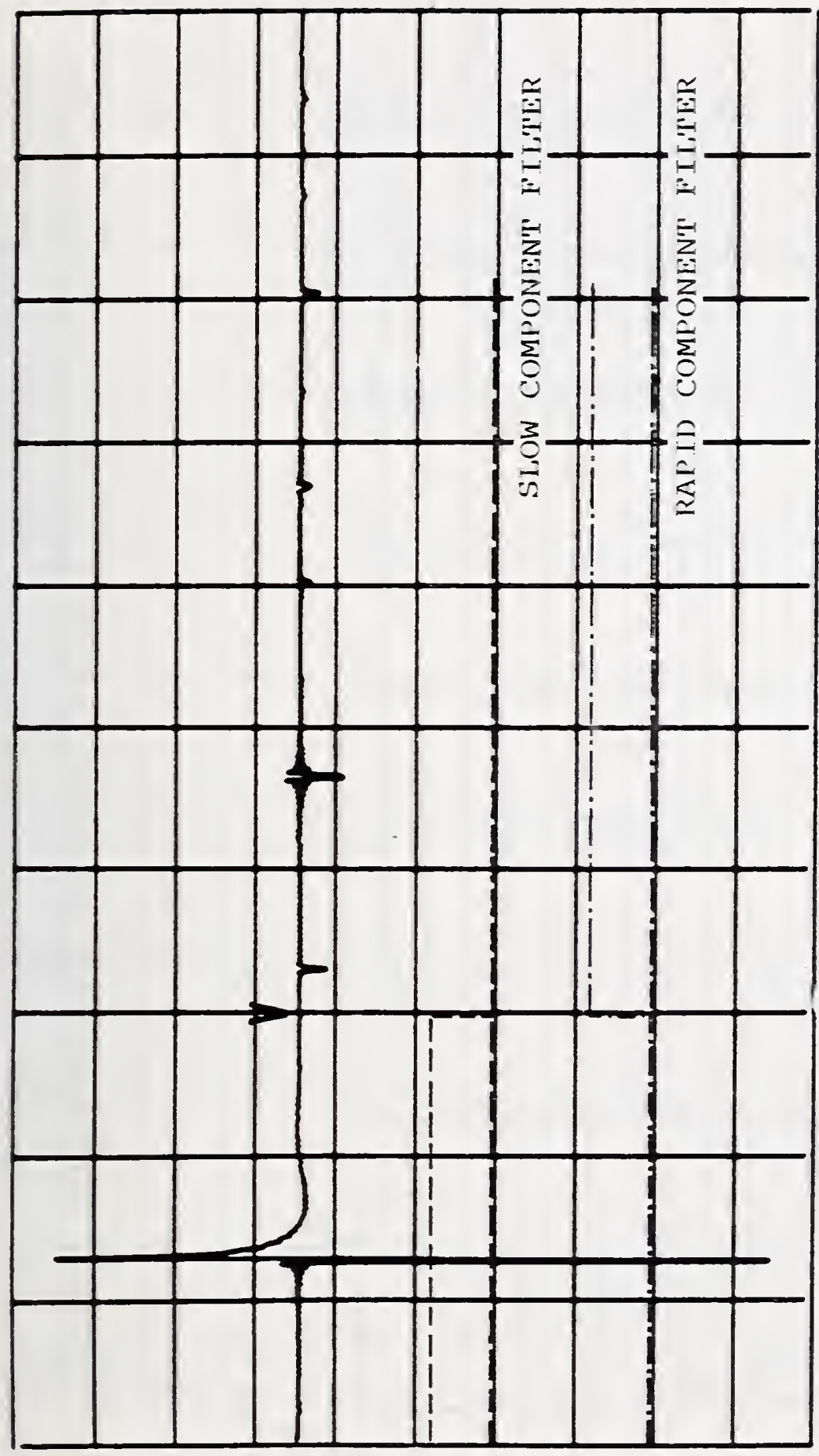

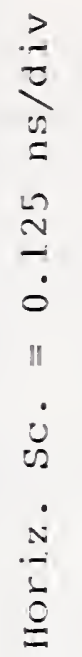

ב⿱一兀)

$\infty$

0
$\vdots$
0
0
0
0
0

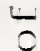

$\Xi$
$\vdots$
0
0
0

-

三 


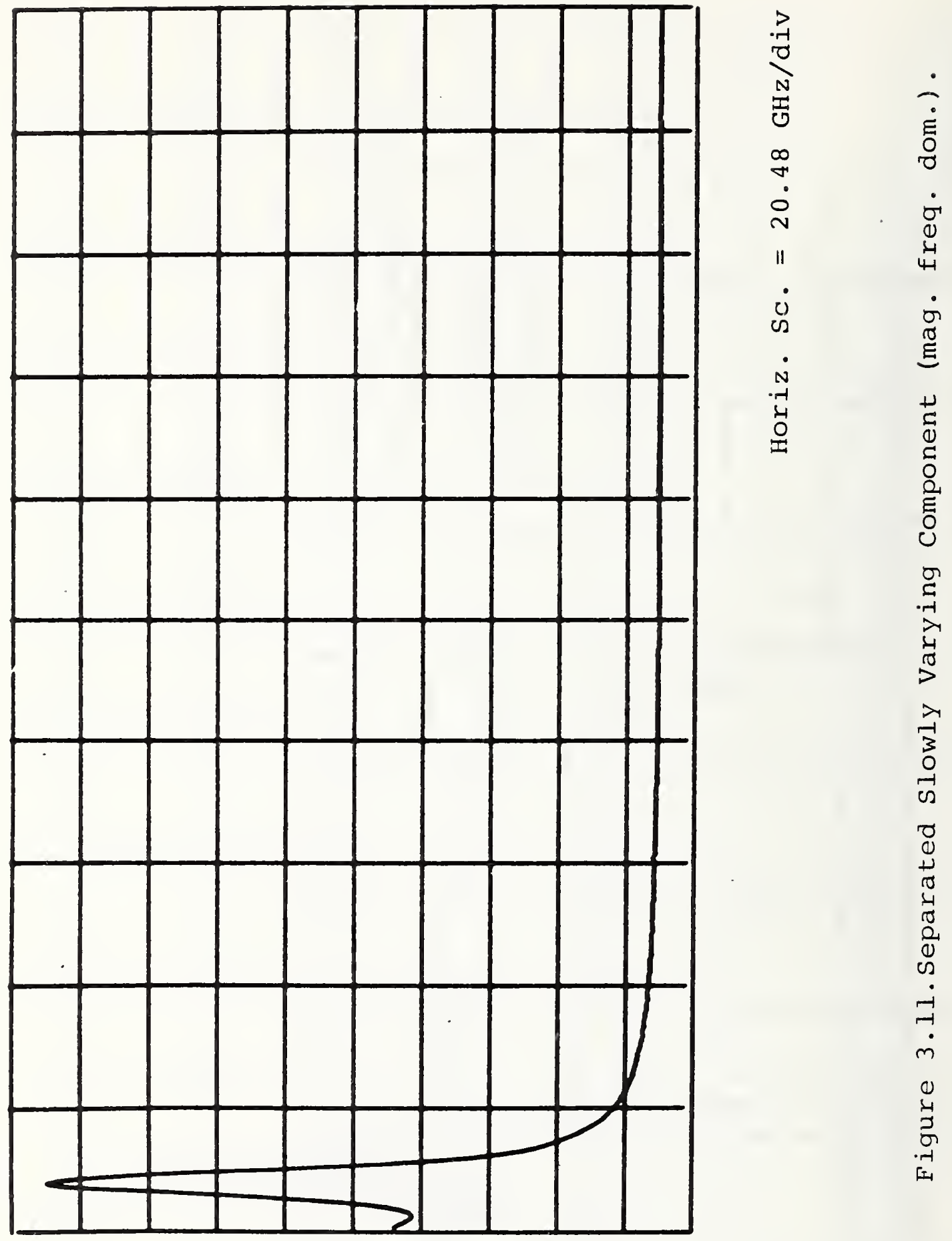




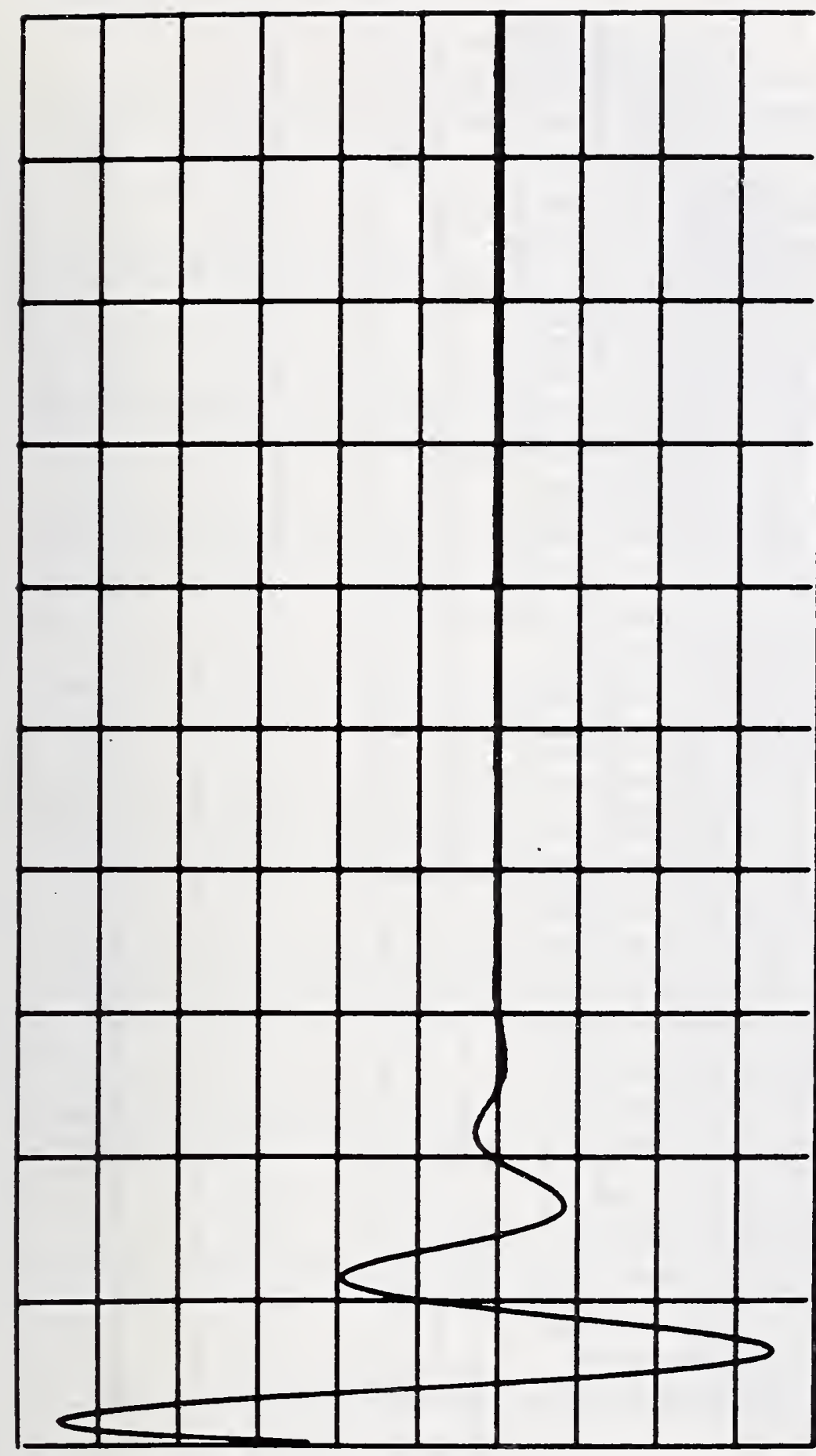

2

กับ

ํํำ

II

ن

$\stackrel{0}{\Xi}$

N

告

ह્.

3
3
0
0
0

0
0
0
0
0
0
0
0

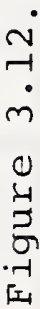




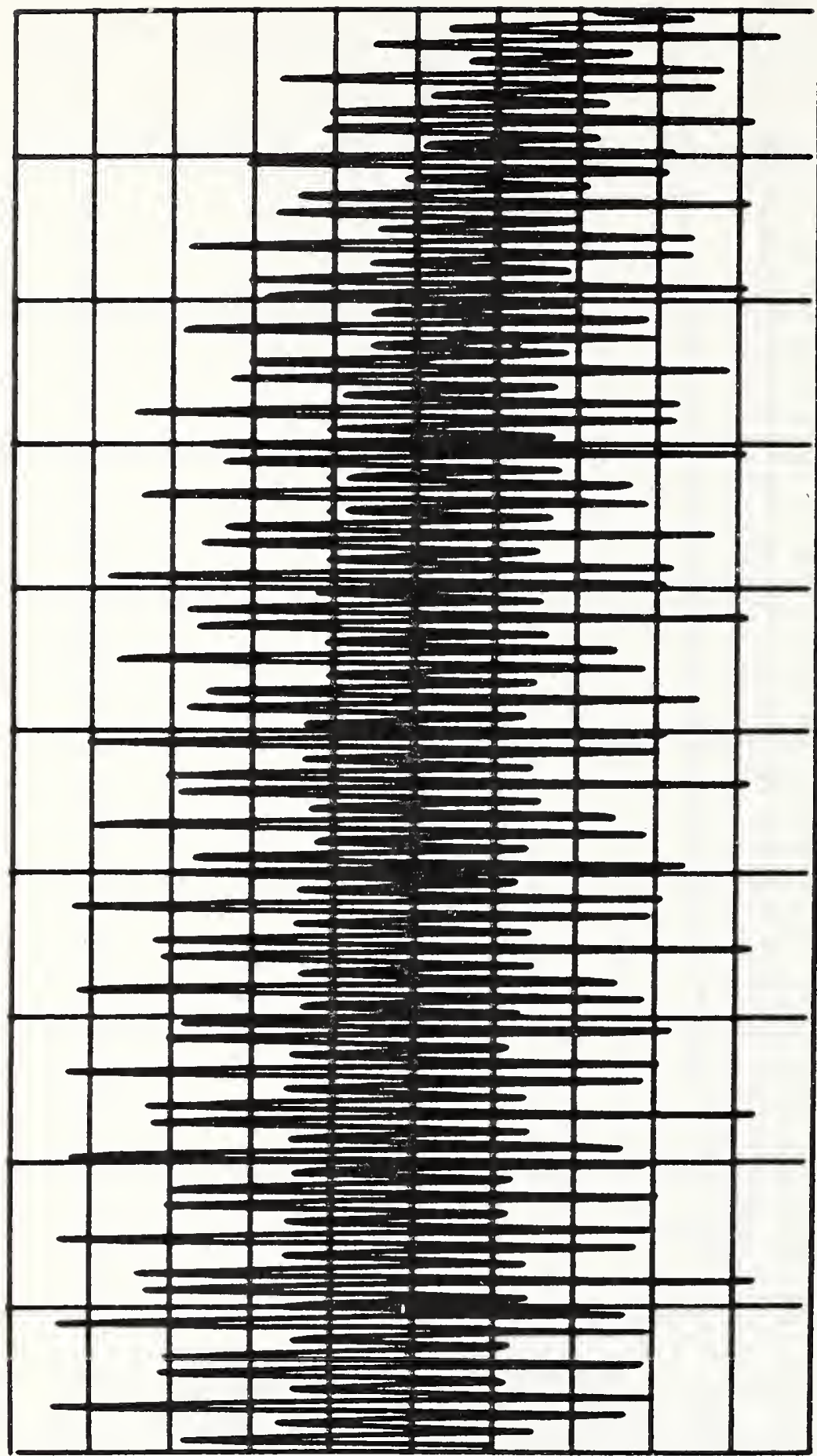

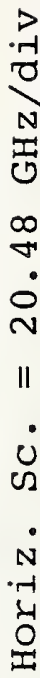

๕̊

$\dot{\sigma}$ 


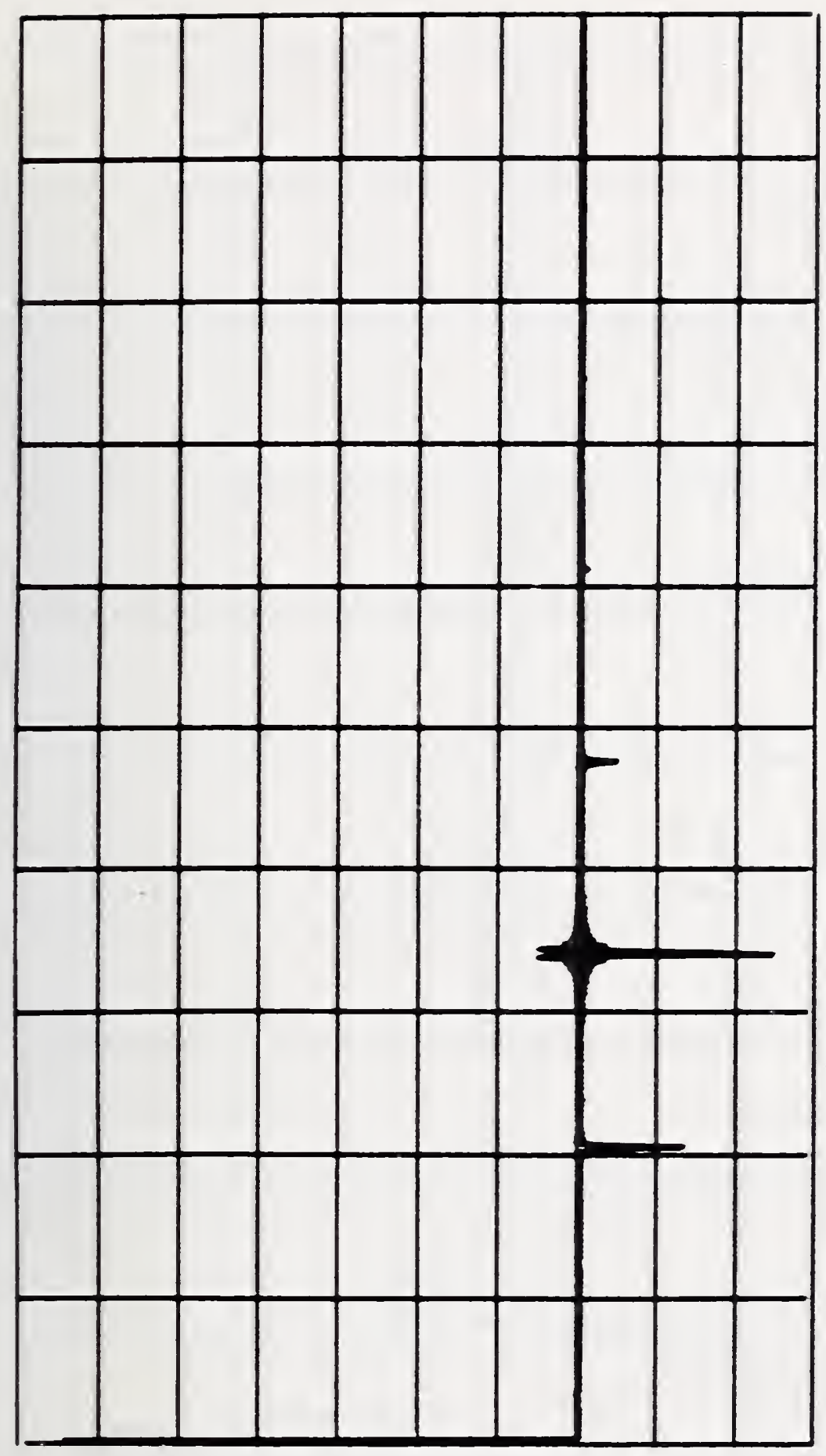

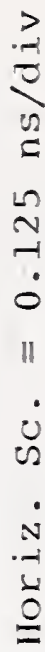

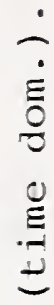

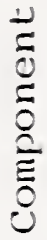

כ)

$\frac{7}{\frac{1}{2}}$

0
0
$\pi$
5
0
0
0
0

$\Xi$
$\dot{\Xi}$
$\vdots$
$\vdots$
$\Xi$ 


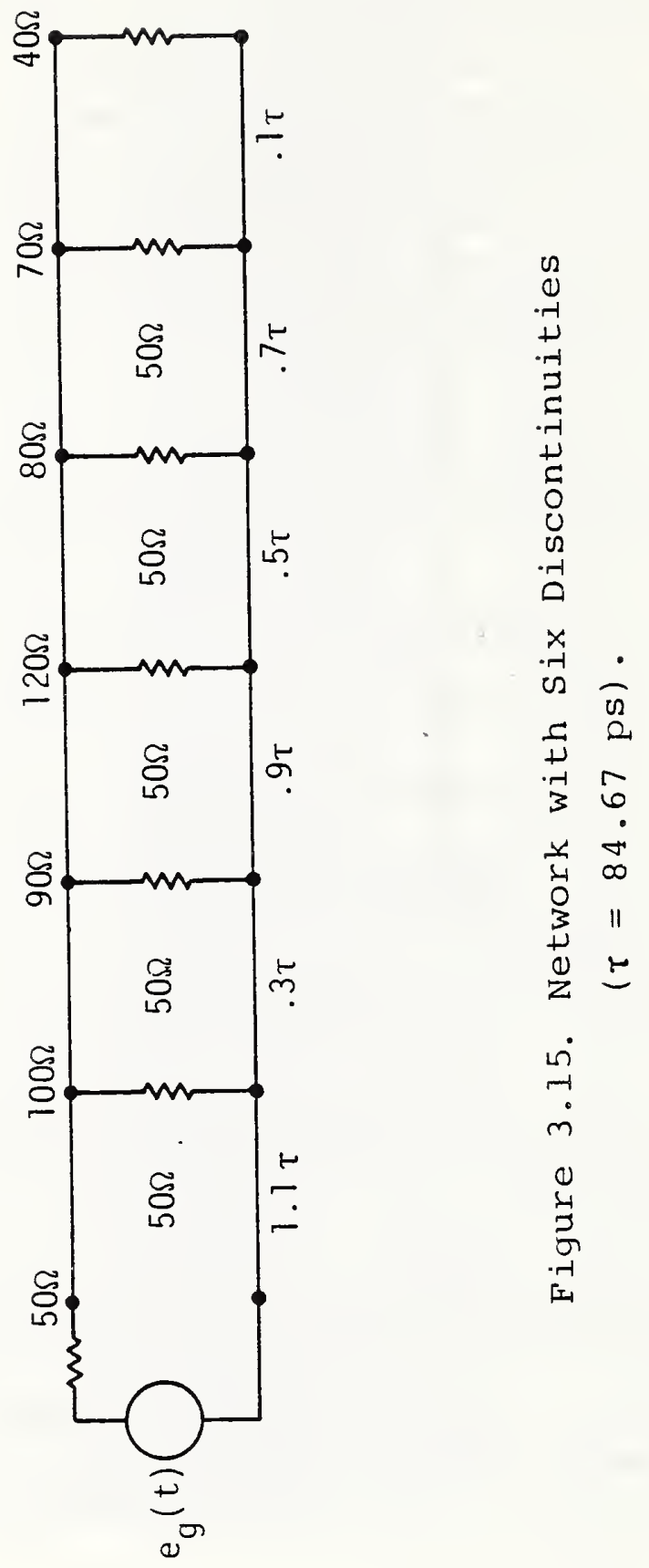



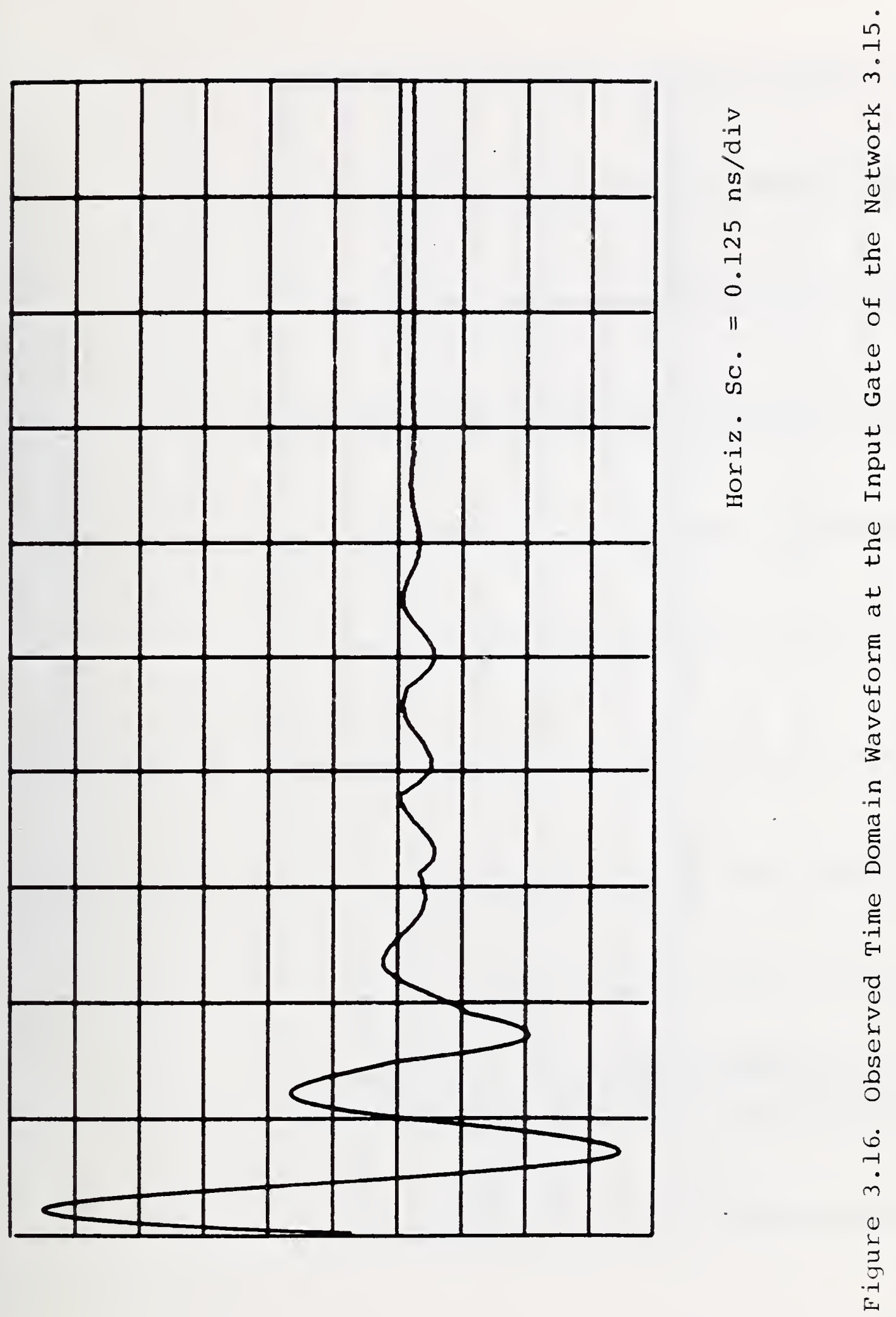


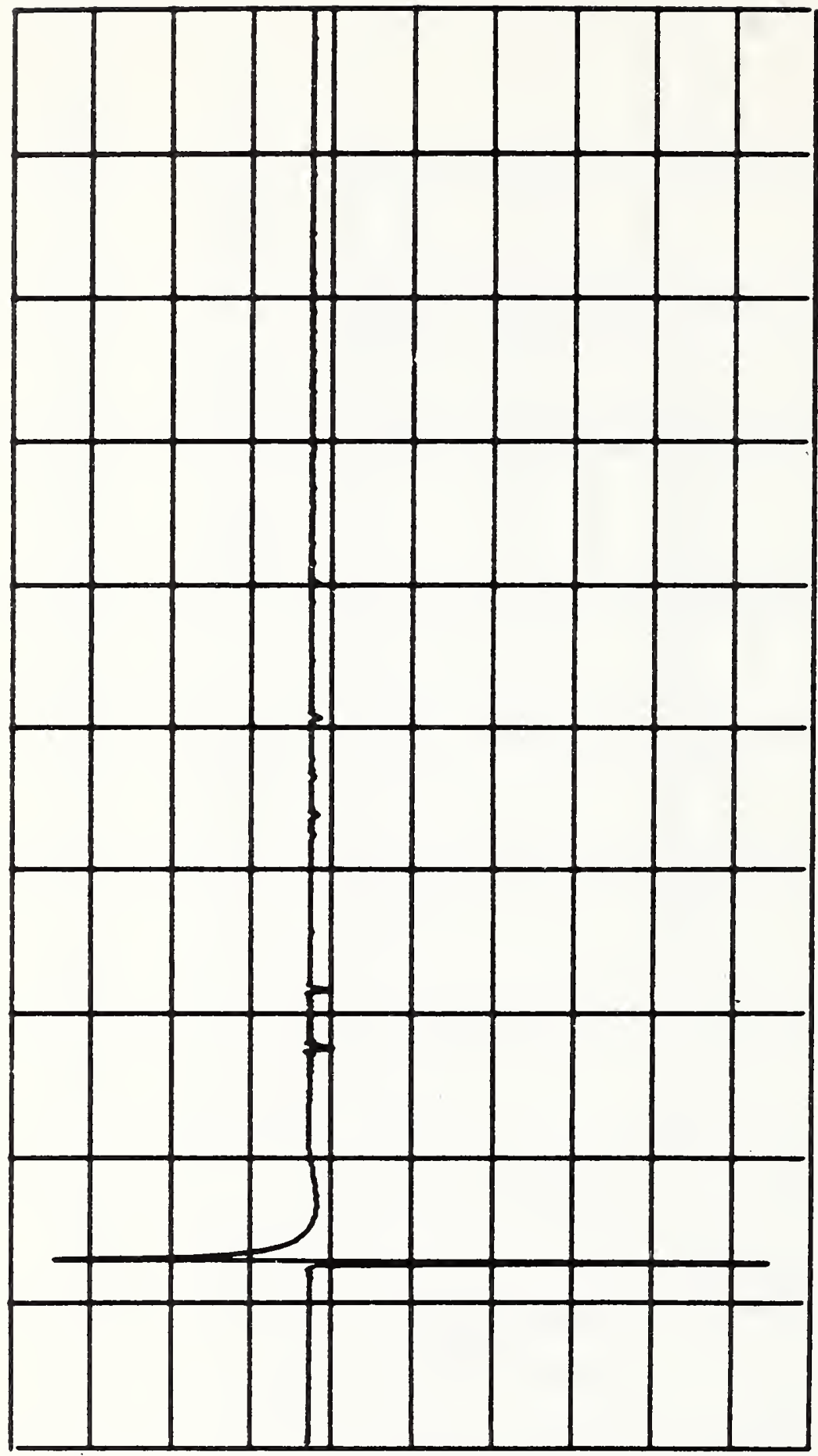

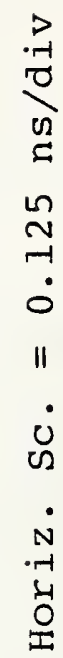

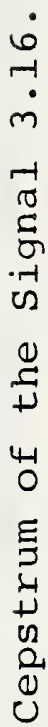

تं 


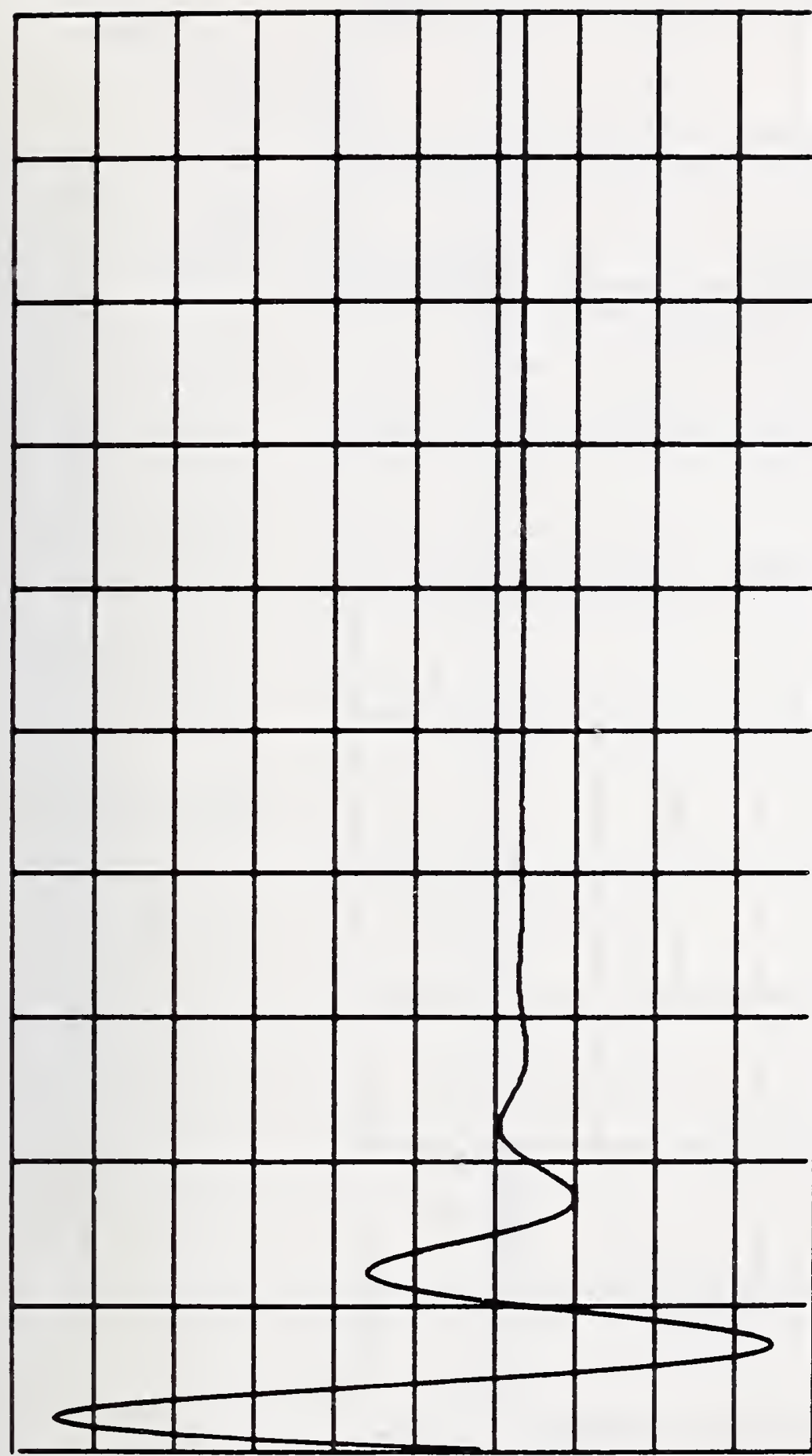

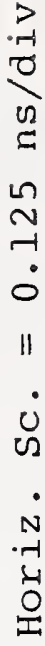

$\stackrel{\bullet}{\stackrel{\bullet}{0}}$

岂

हू
0
0
0
0
0
0

E్

$\lambda$
-1
3
0
-1
0

0
0
0
0
0
0
0
0
0
0

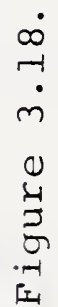




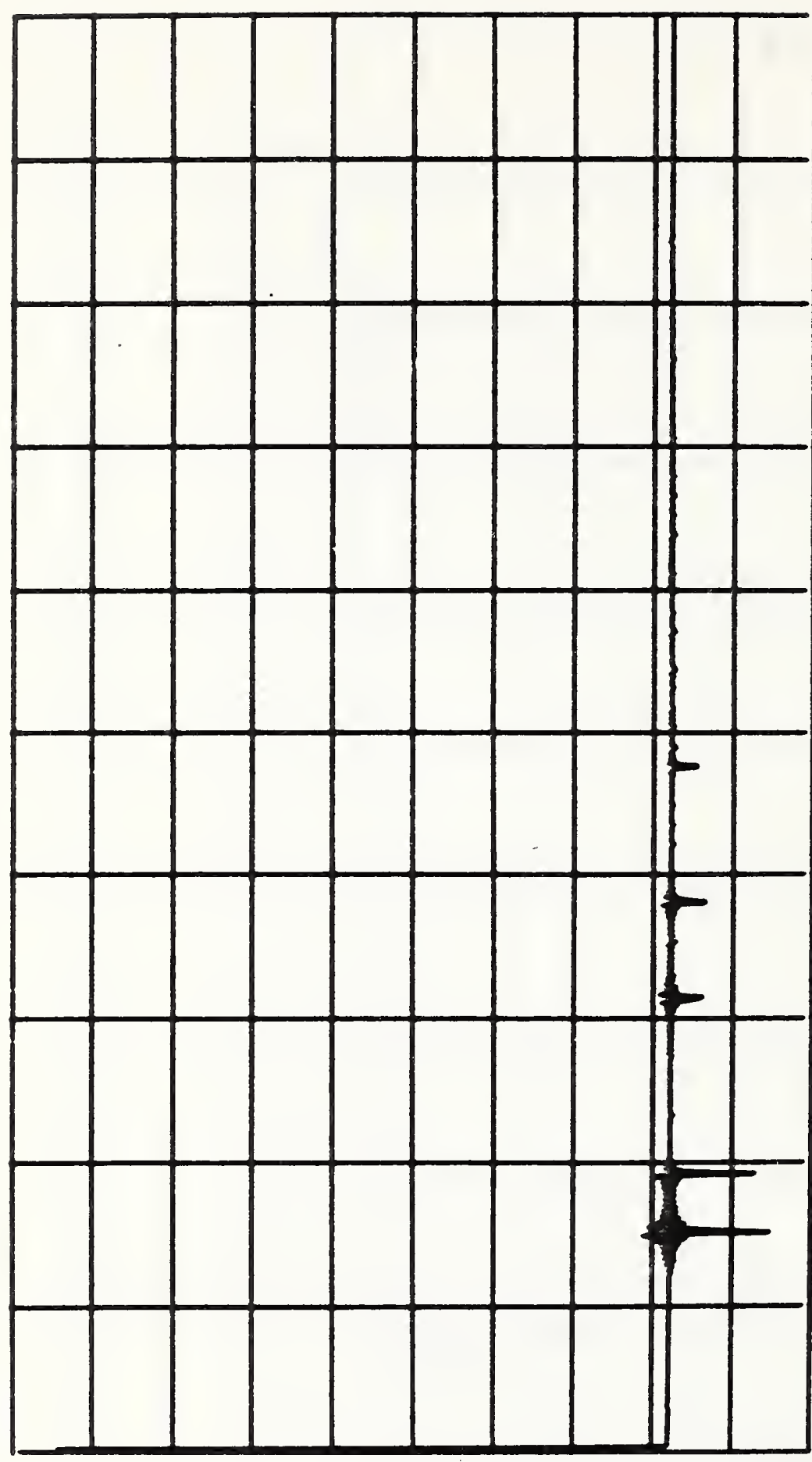

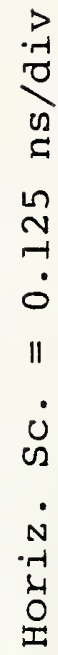

$\stackrel{2}{\dot{0}}$

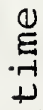

Ů

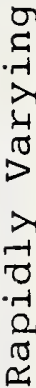

0
0
+1
0
0
0
0
0
0

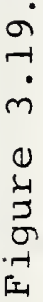


a. NETWORK

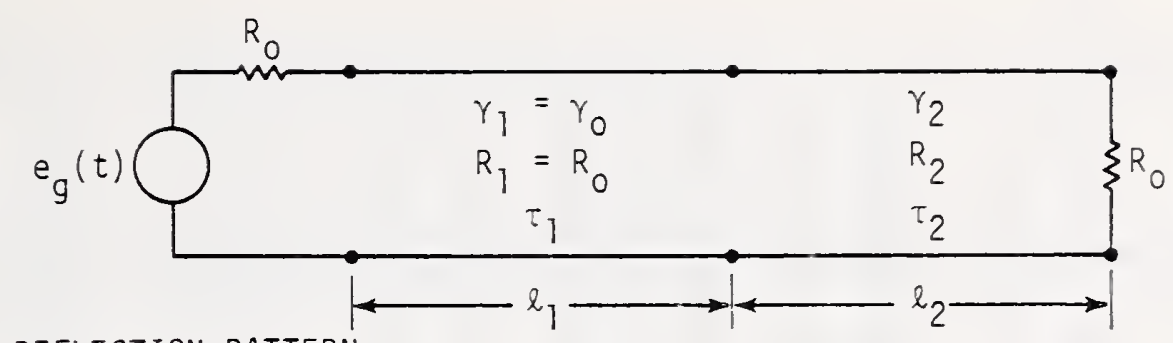

b. MULTIPLE REFLECTION PATTERN

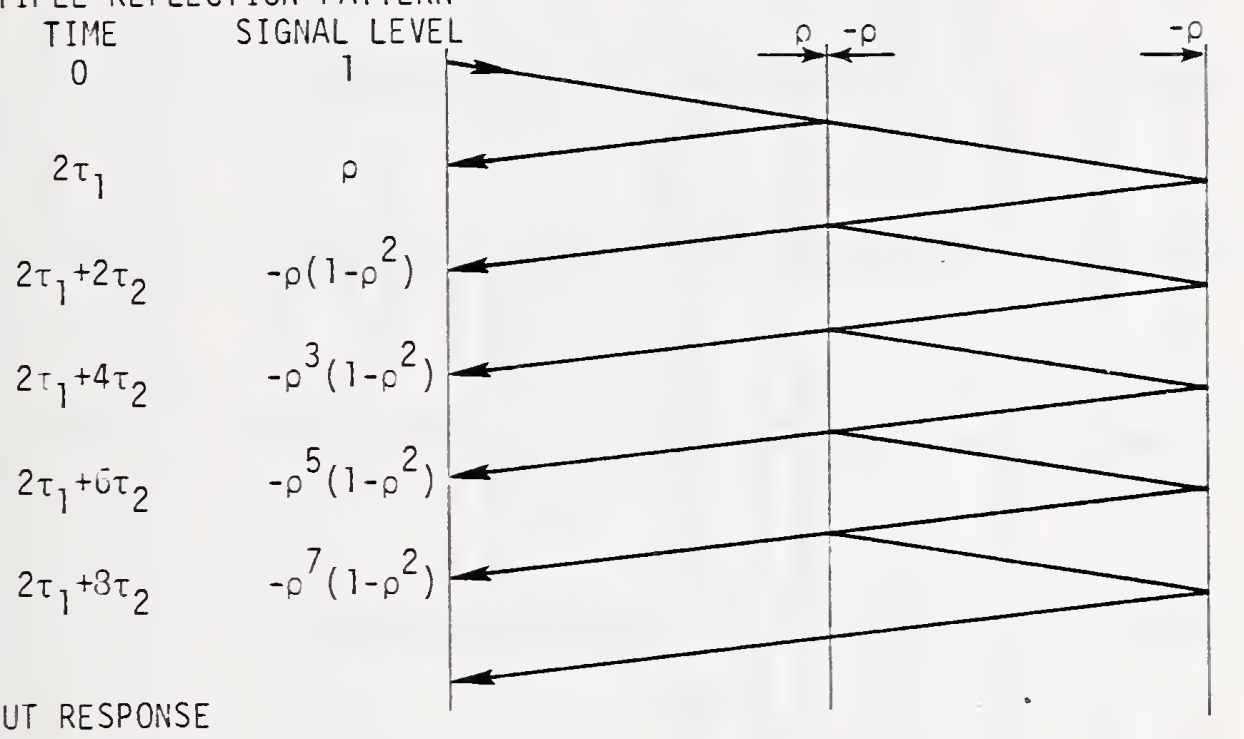

c. INPUT RESPONSE

$$
\begin{aligned}
& h(t)=\delta(t)+h^{\prime}(t) \\
& \rho=1 / 3 \\
& \tau_{1}=1.5 \tau, \\
& \tau_{2}=1.2 \tau \\
& \text { WHERE } T=84.6 \mathrm{ps}
\end{aligned}
$$

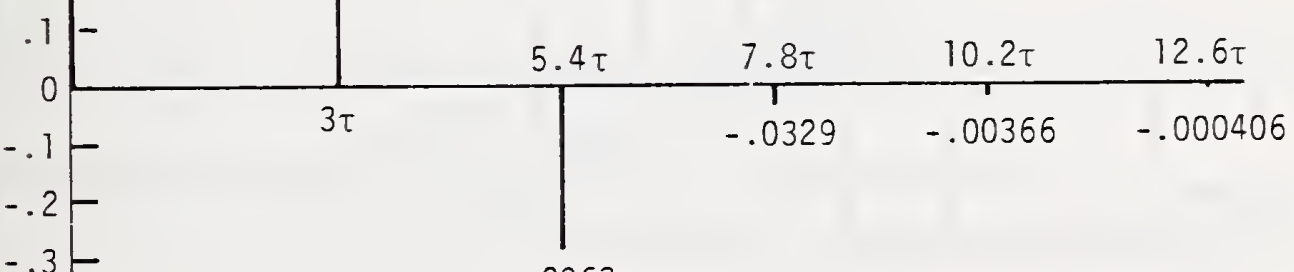

$-.3-$

$-.4-$

$-.5 L$

Figure 3.20. Case of Two Commensurate Transmission Iines with Two Discontinuities. 


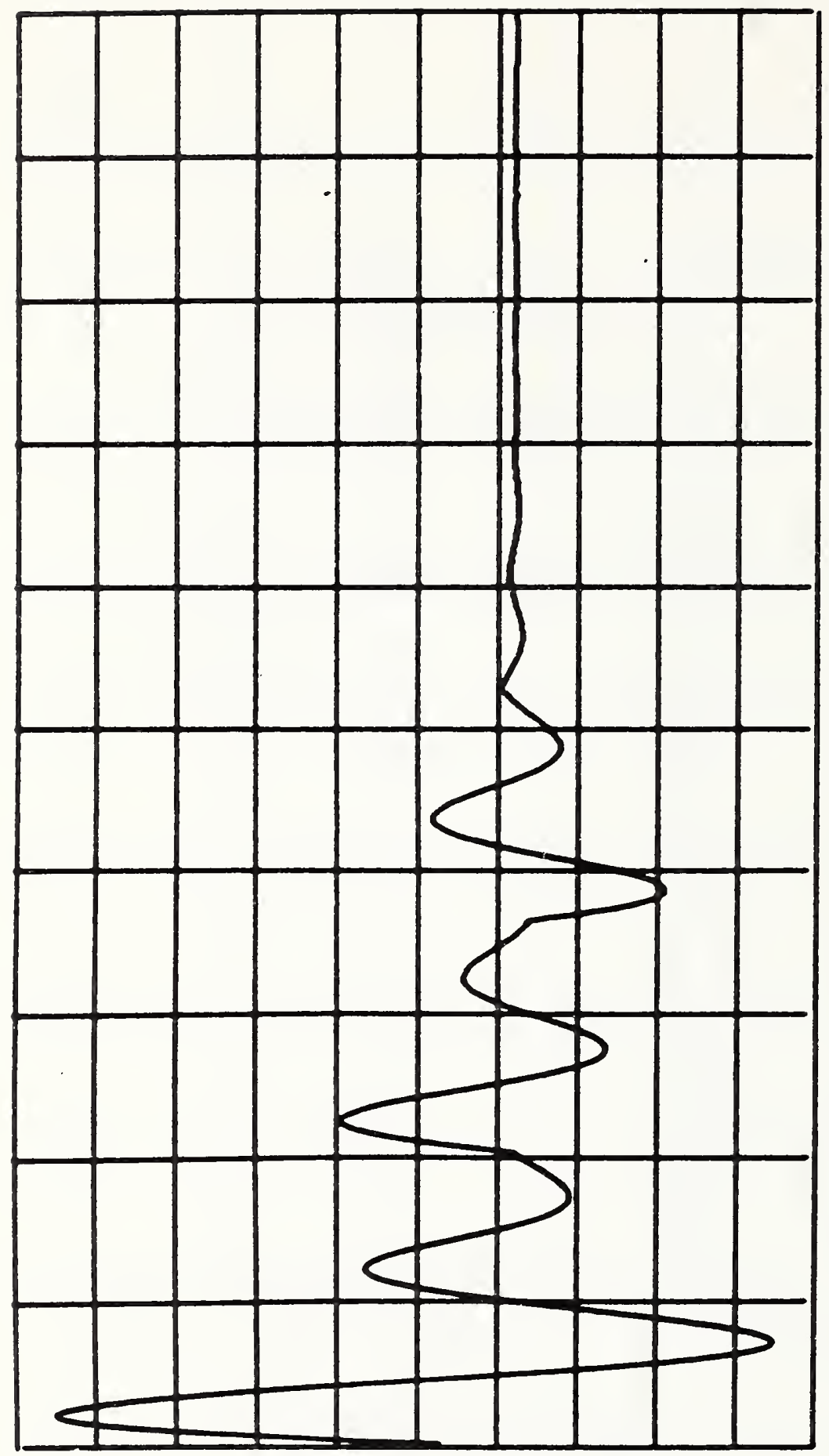

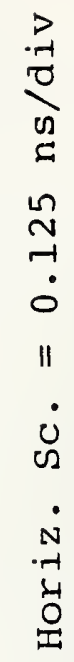

m

0

हु

[

4

步

3

(1)

它

4

0
+
0

لِ

$+$

4

2

3

0
0
2
0
0
0
0
0

-

r

$m$

0
.1
2
0
-1
I. 


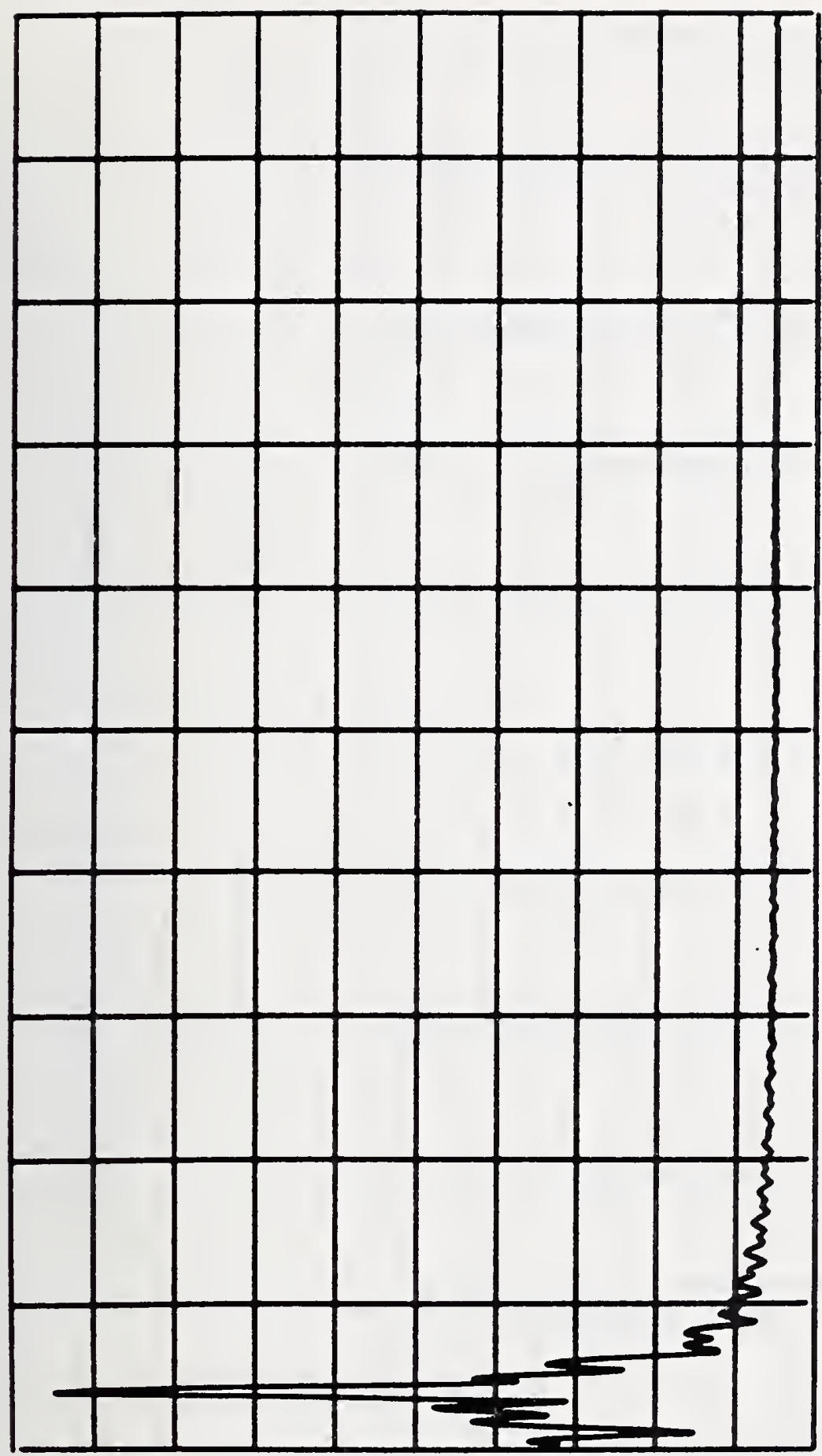

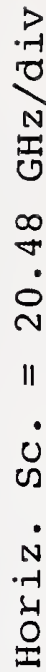

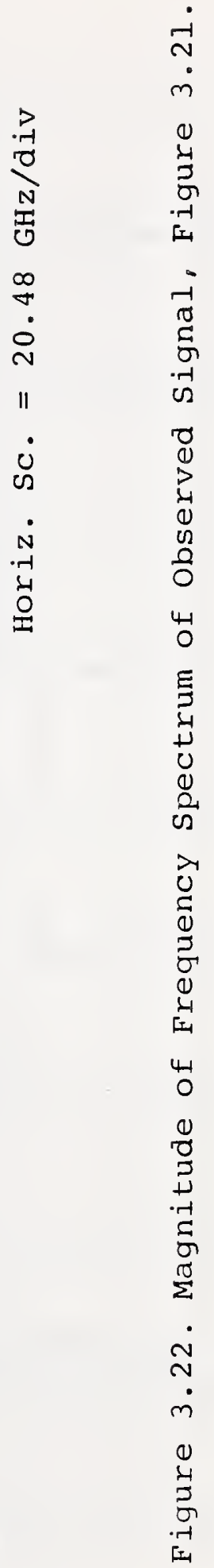




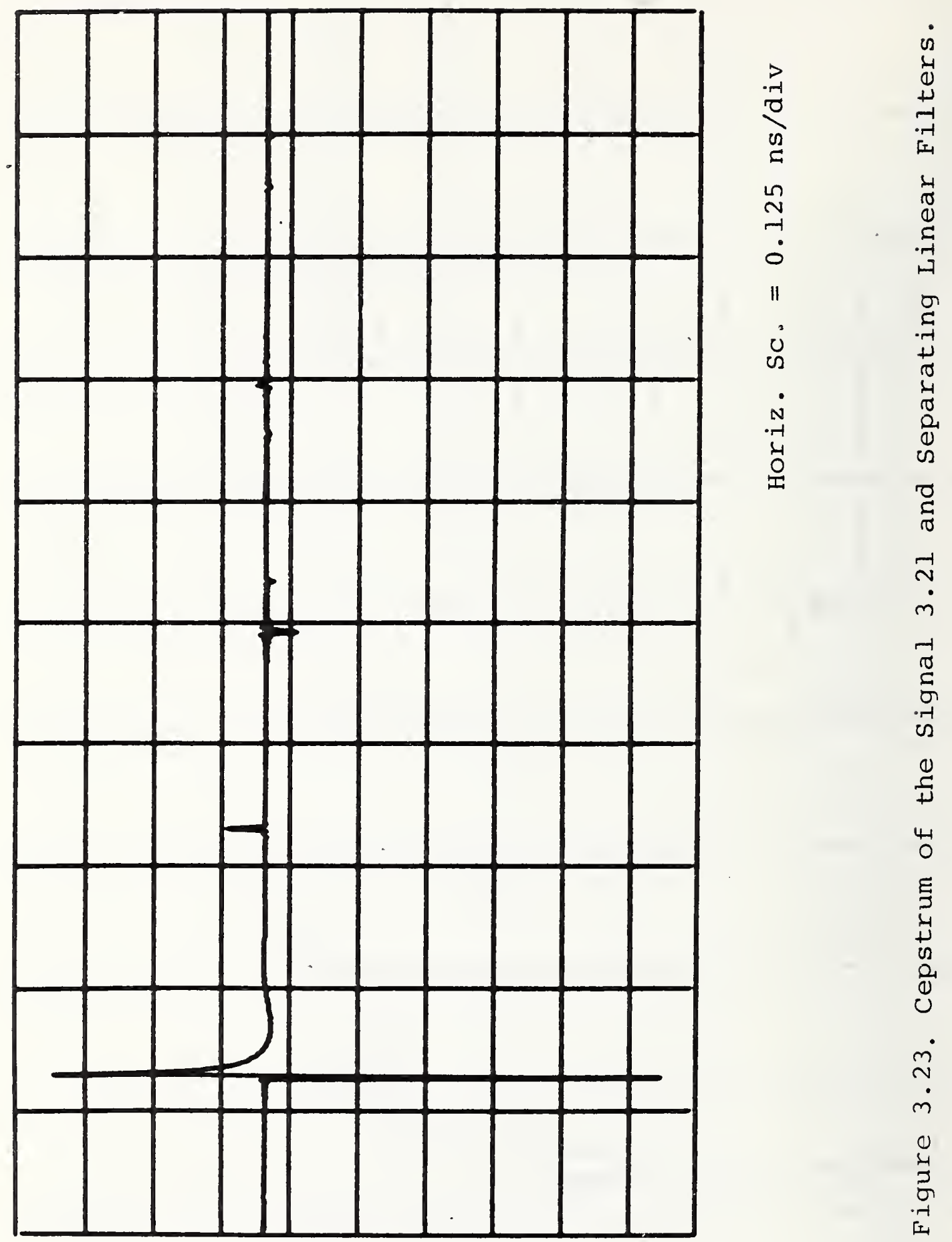




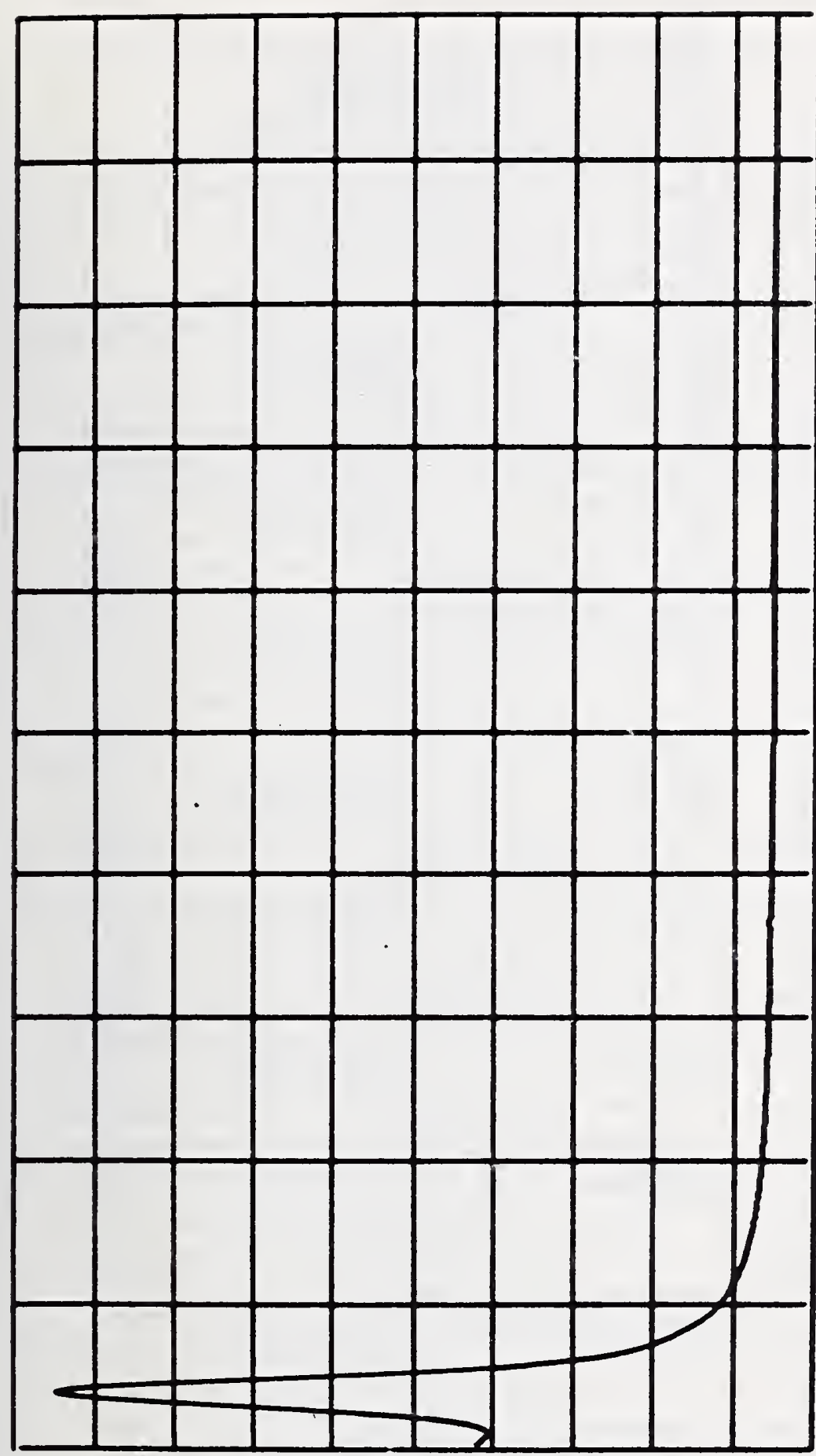

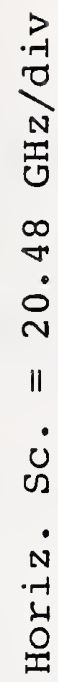

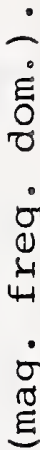

ฮั)

त्न

-1
3
0
0
01

రิ

$+$

b

$\frac{1}{0}$

os

ัำ

$\dot{m}$

崩 


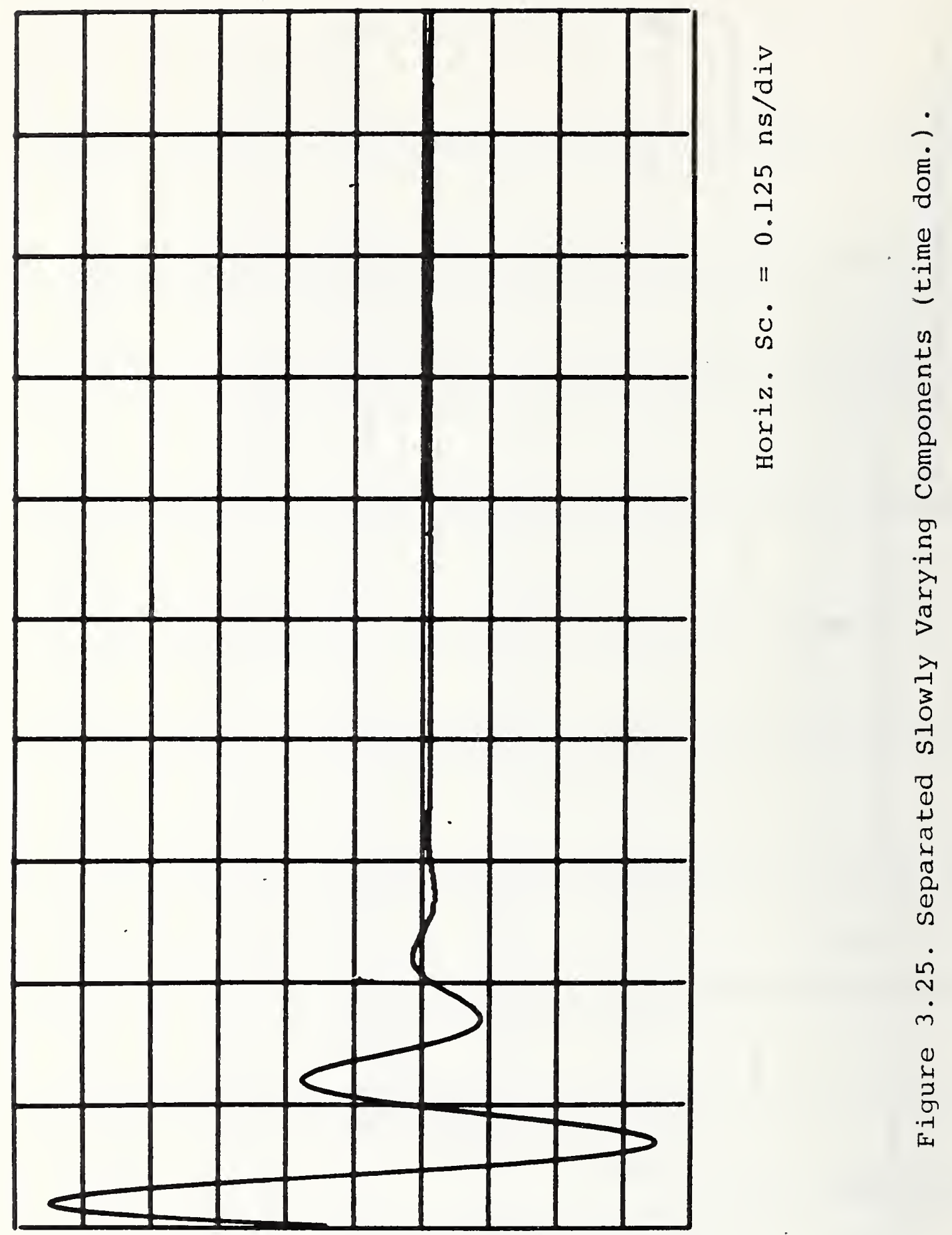




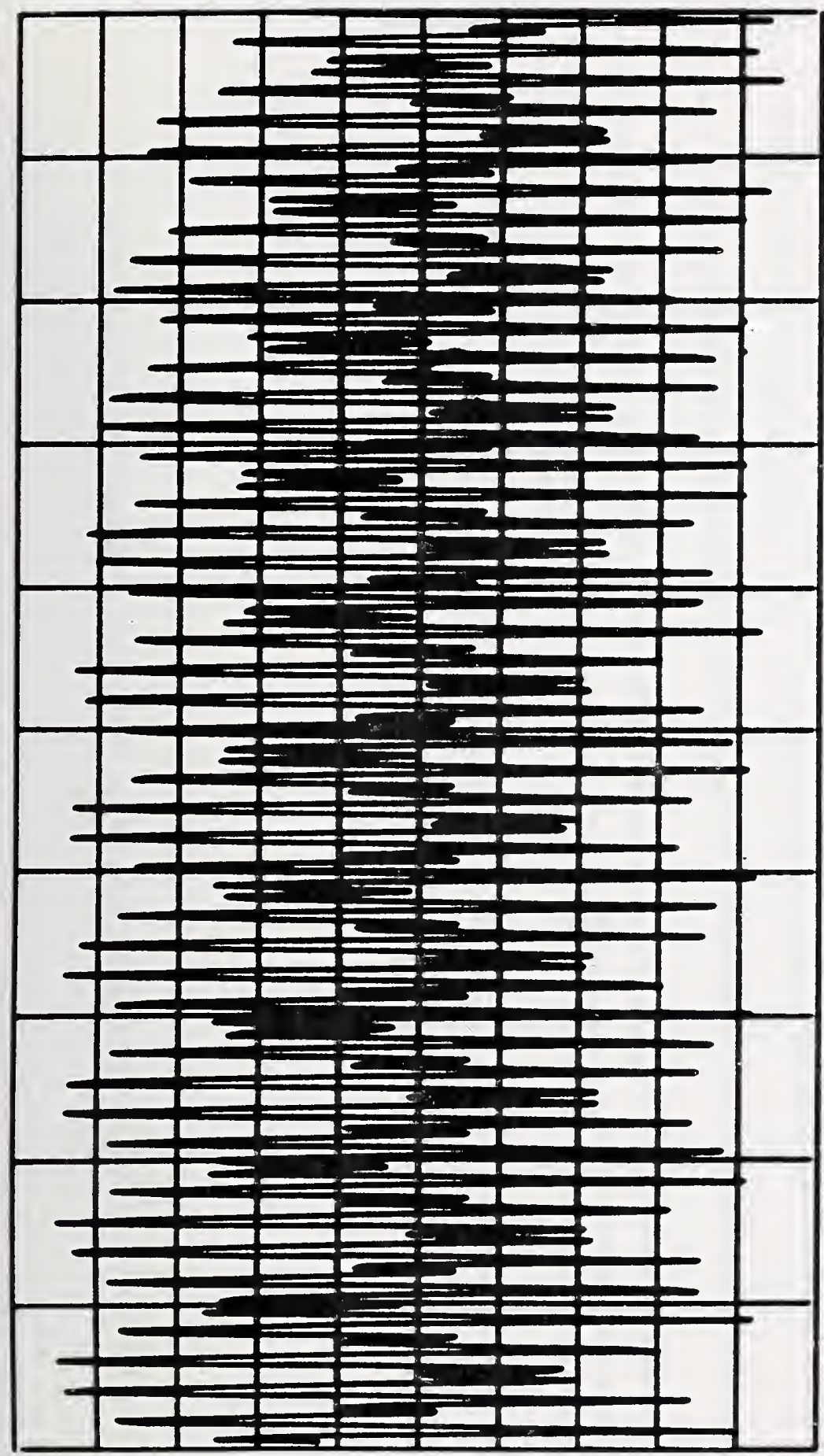

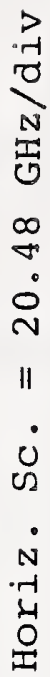

ن 


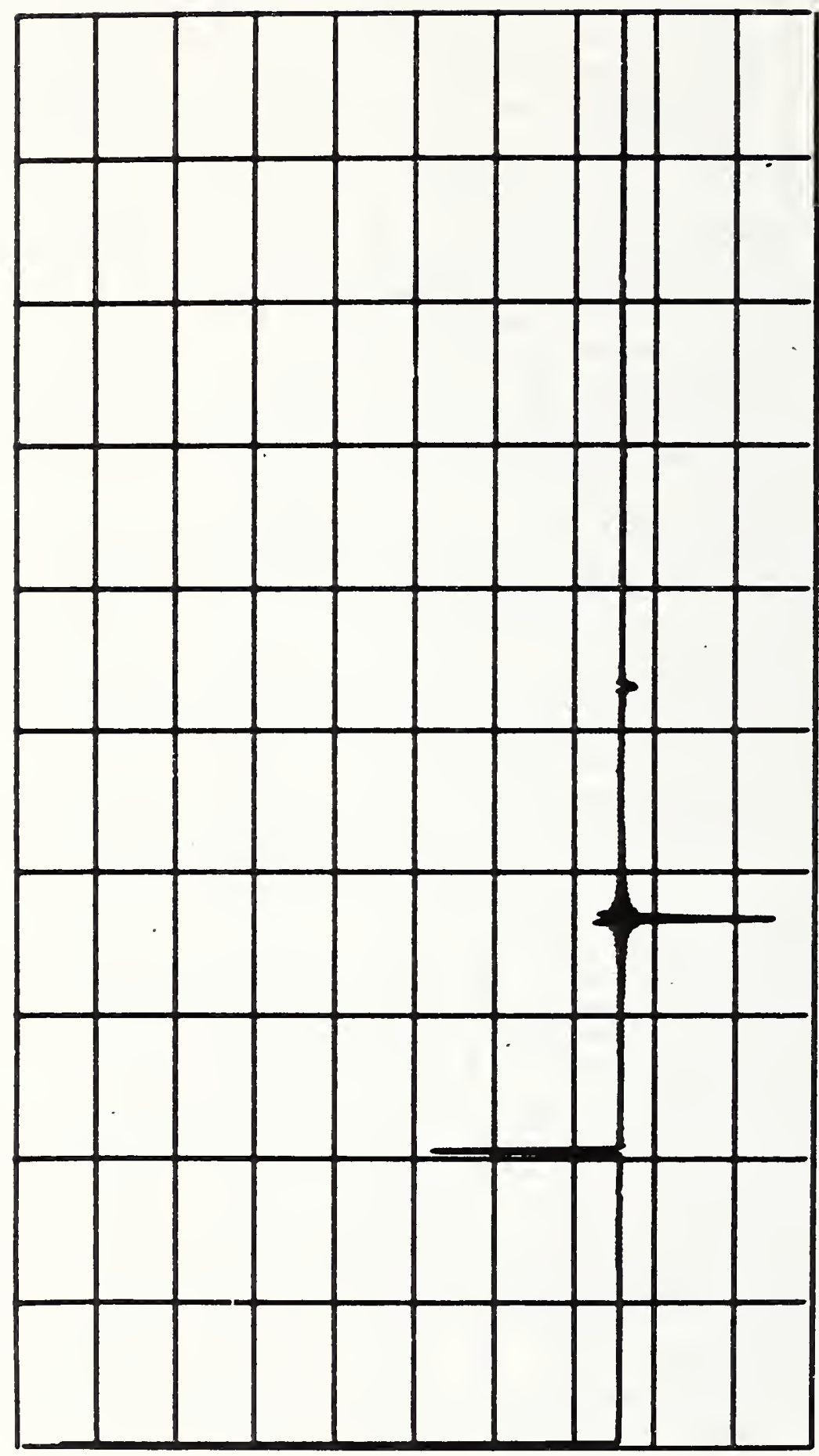

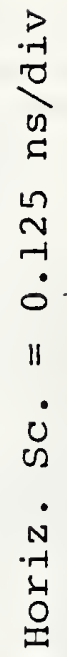

룽

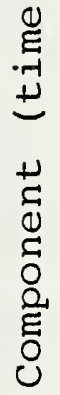

包

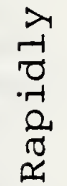

0
0
+1
0
4
0
0
0
$\Omega$

N

?

岌 


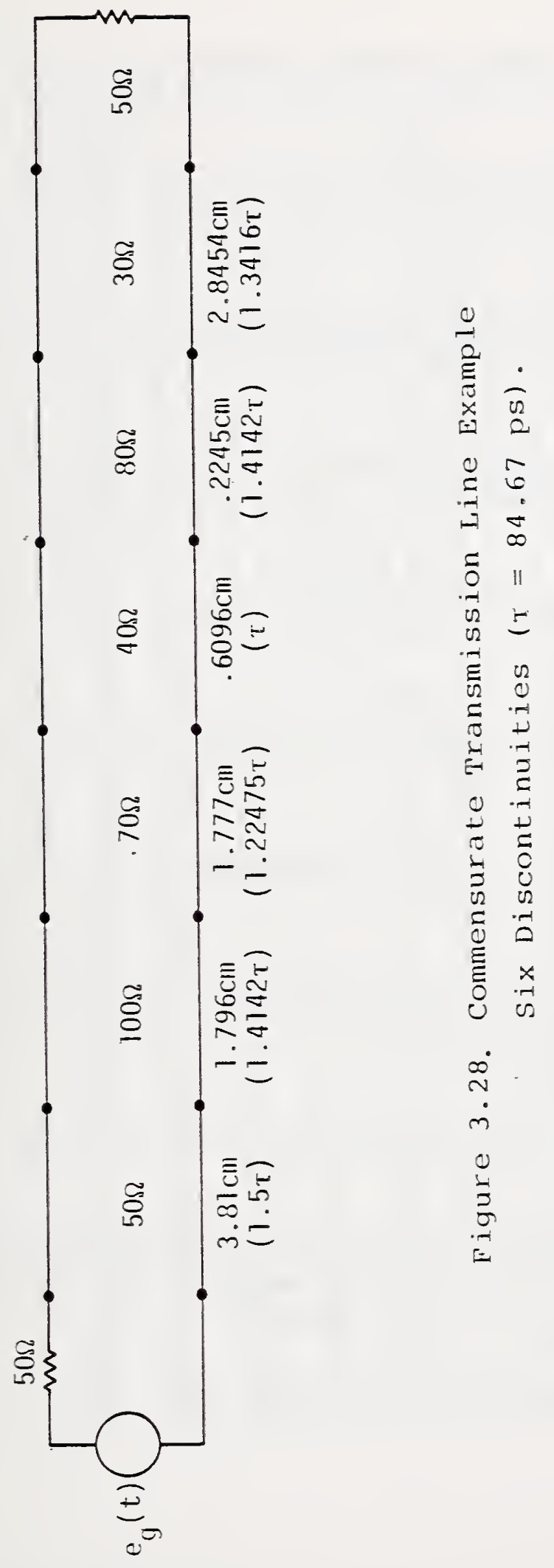




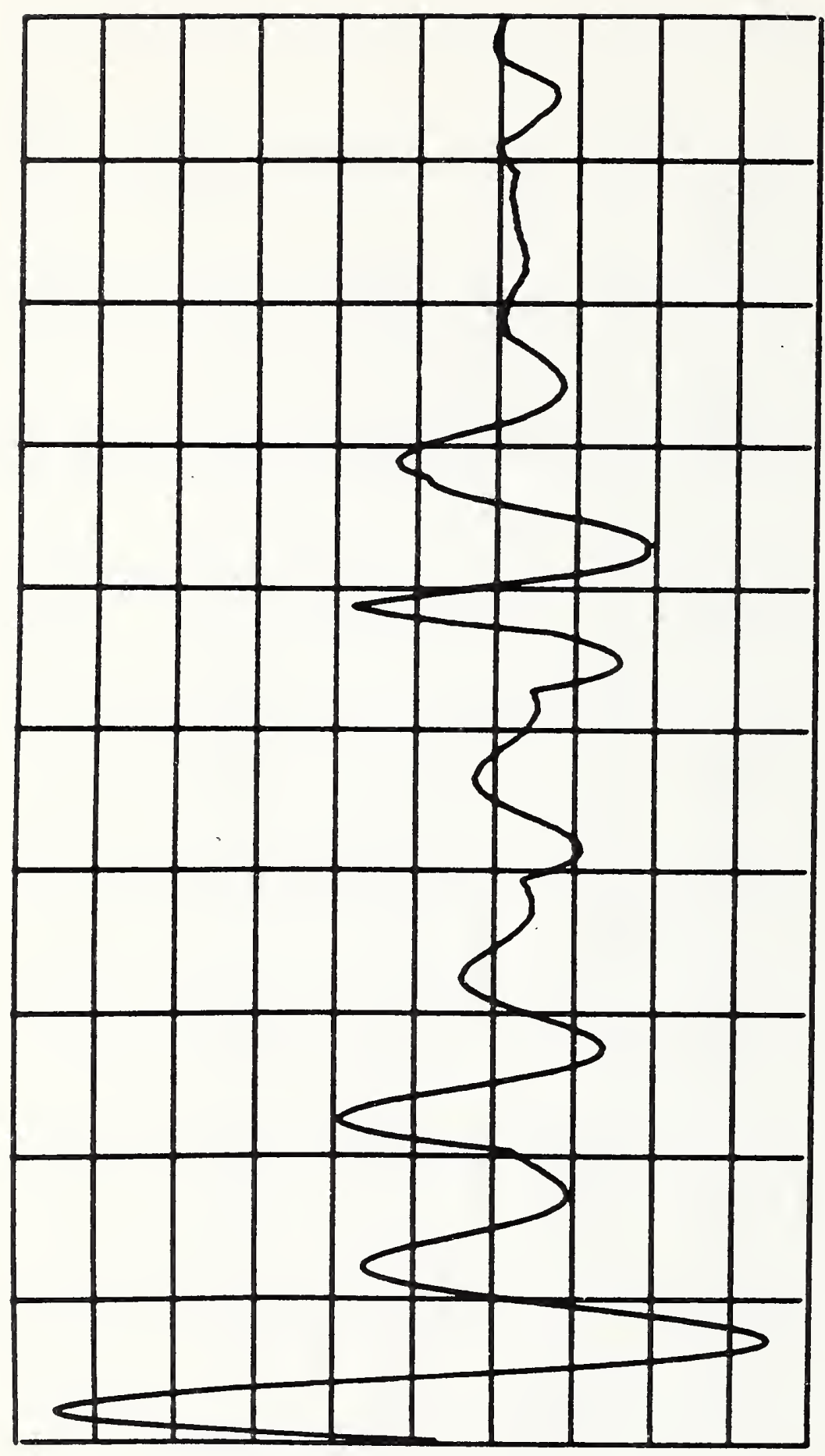

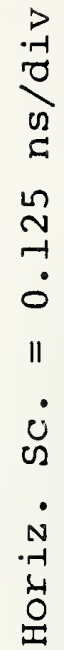

3
0
3
4
1
2

4

(1)

茂

峁

론

t)

E

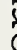

4

2

3

.

害

0
.7
E-
0
0
0
0
0
0
0
0

a

$v$

$m$

㟔 


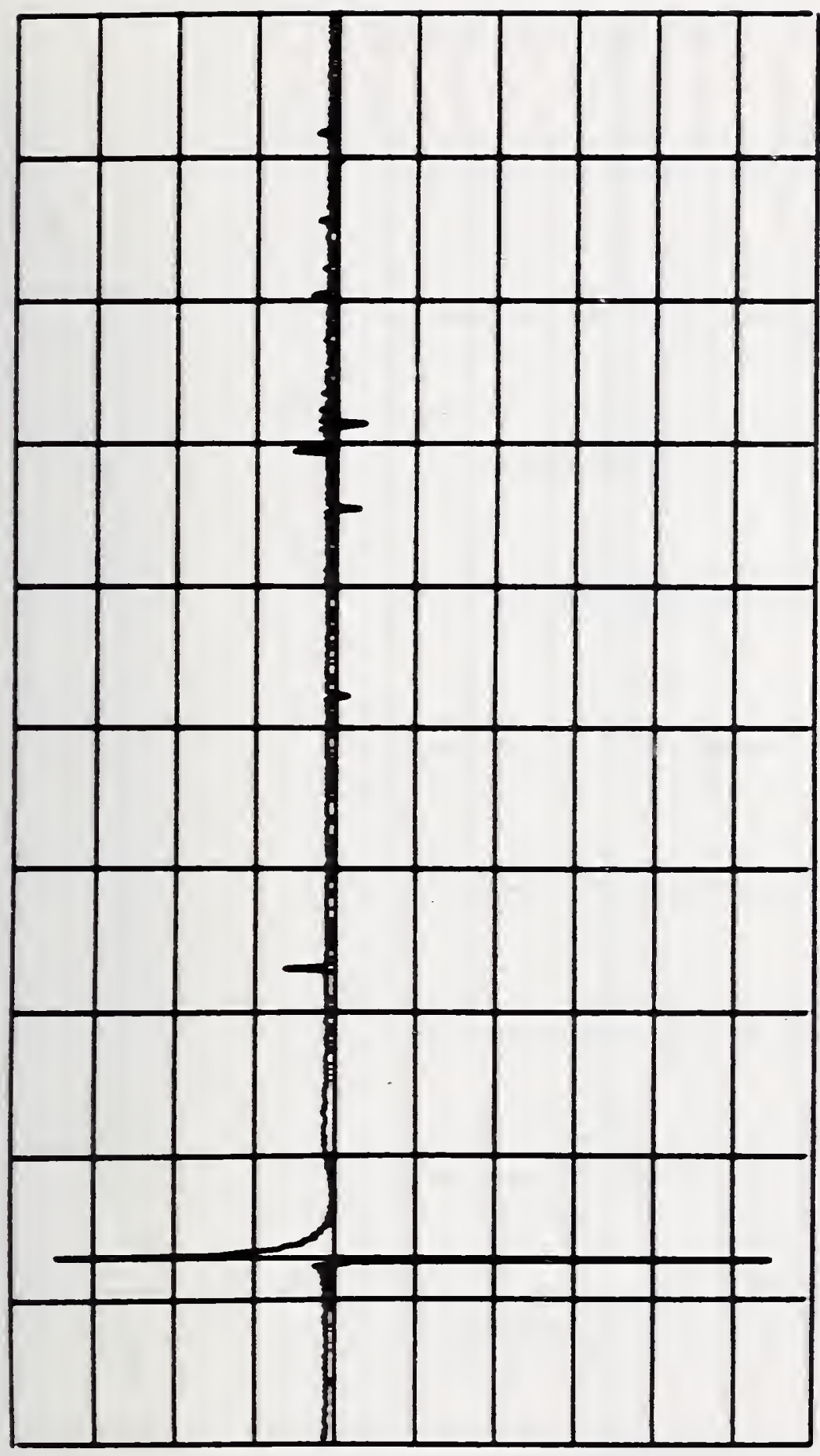

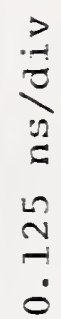

II

ن $\quad \dot{m}$

$\stackrel{-1}{\stackrel{N}{0}}$

ป

0

ह

웅

$\dot{m}$

בְ 


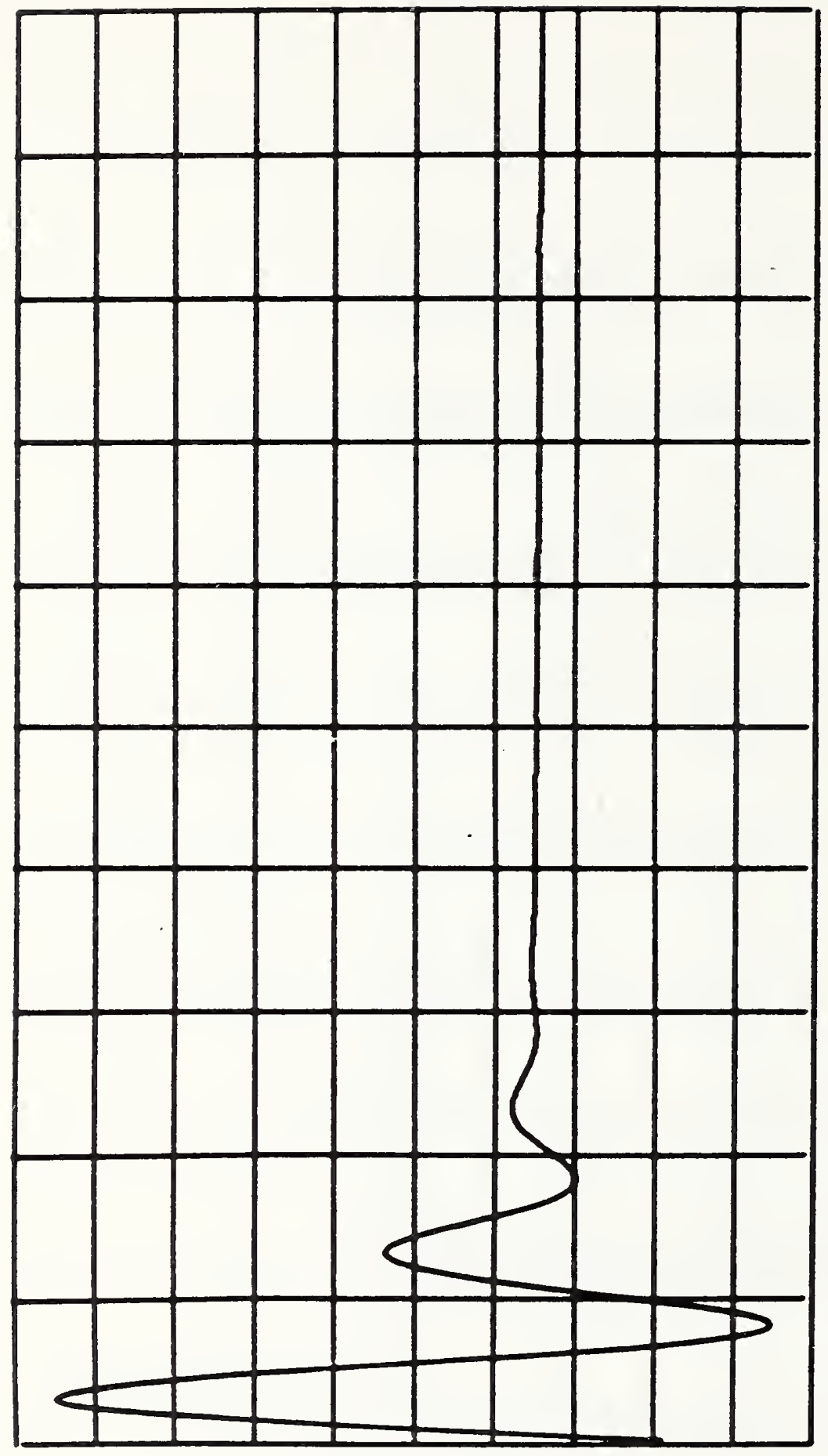

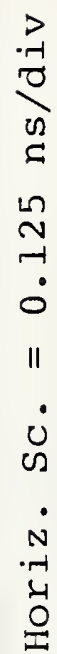

ت

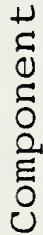



7
3
0
0
0
0
0
0
0
0
0
0
0
0

ले 


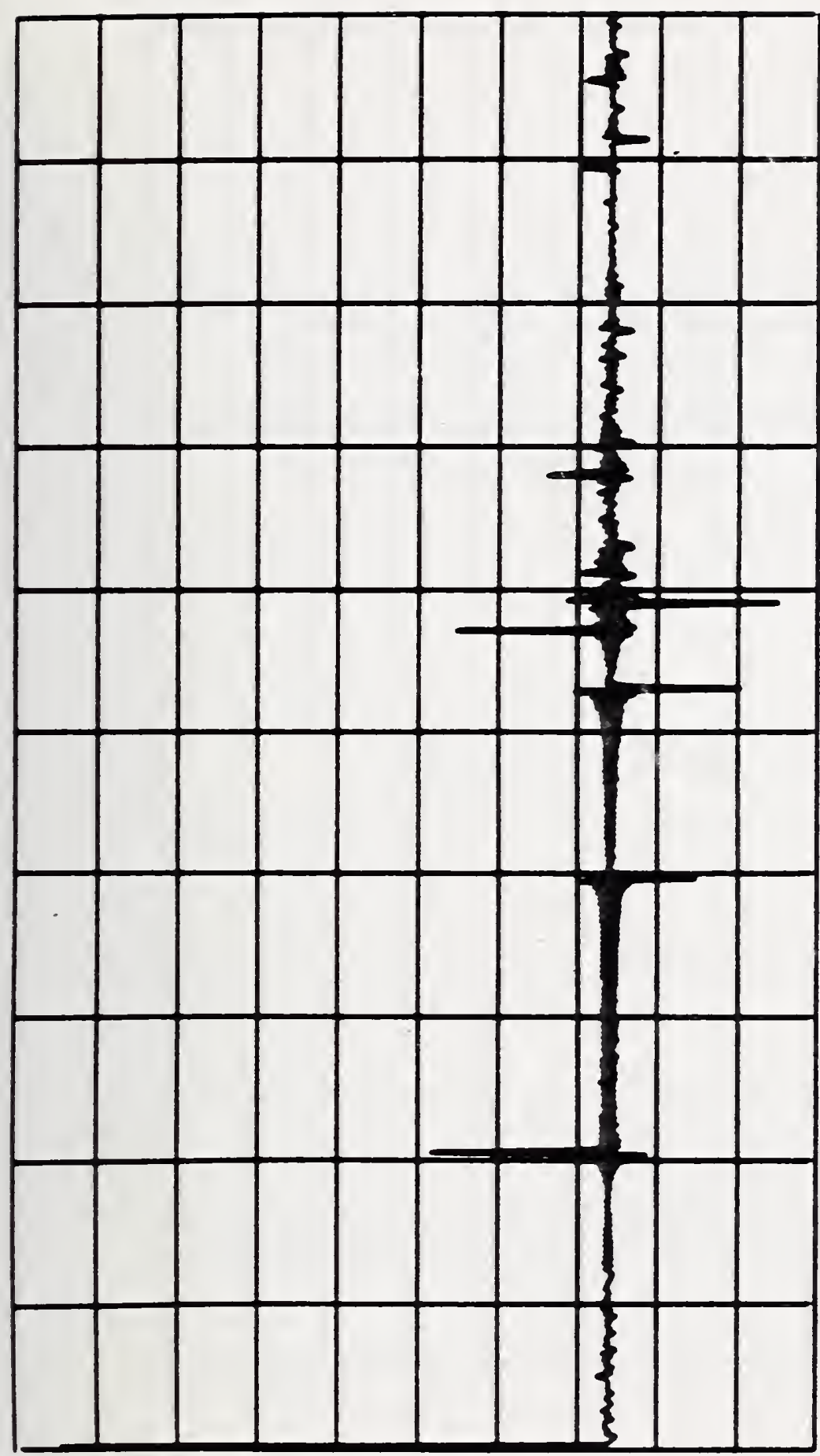

ב

先

굴

0
0
$\pi$
0
0
0
0
0
0

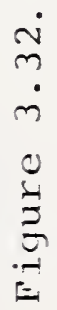




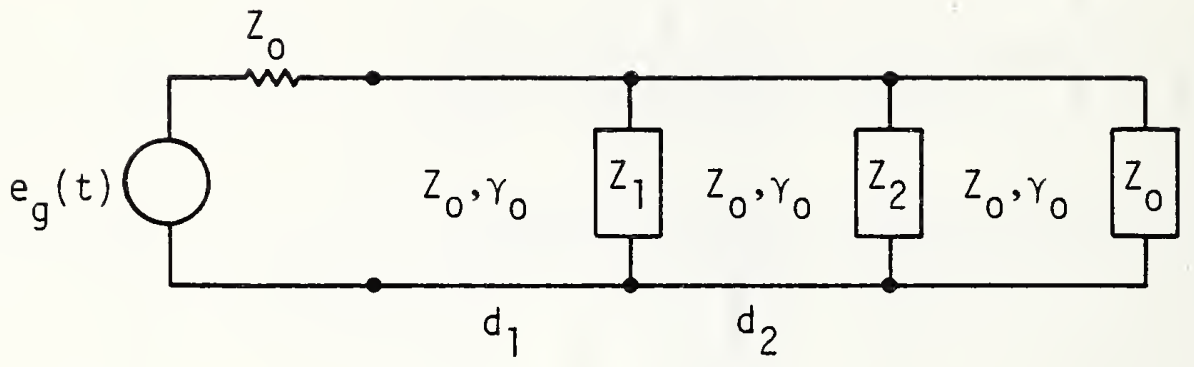

Figure 3.33. TDT Network. 


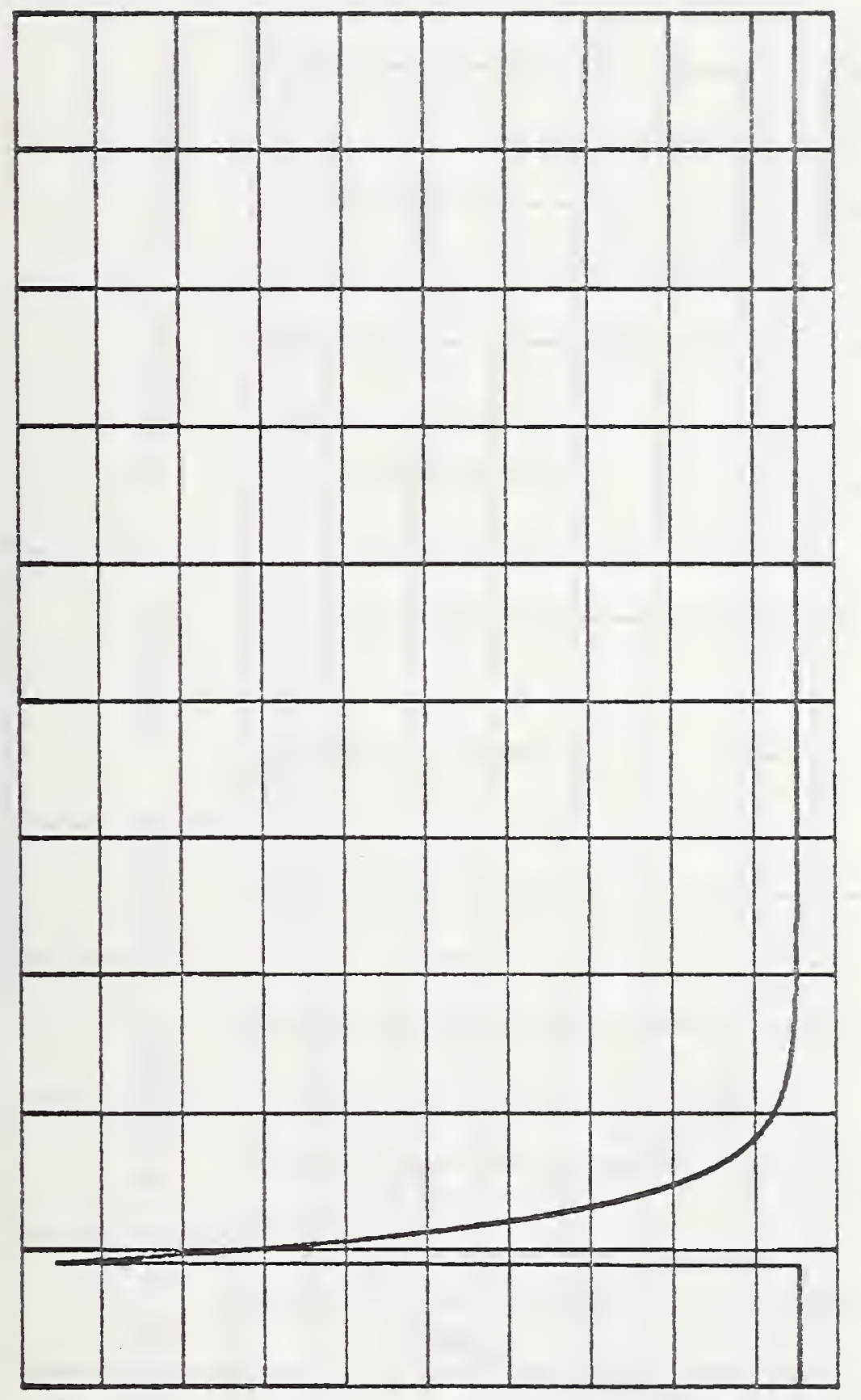

20

I

0

톨

용

.

हू

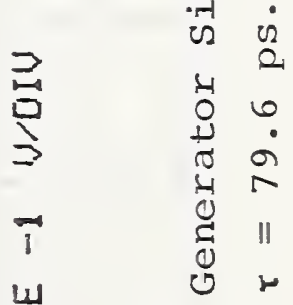

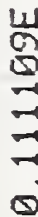

11
0
$\frac{1}{3}$

लं 


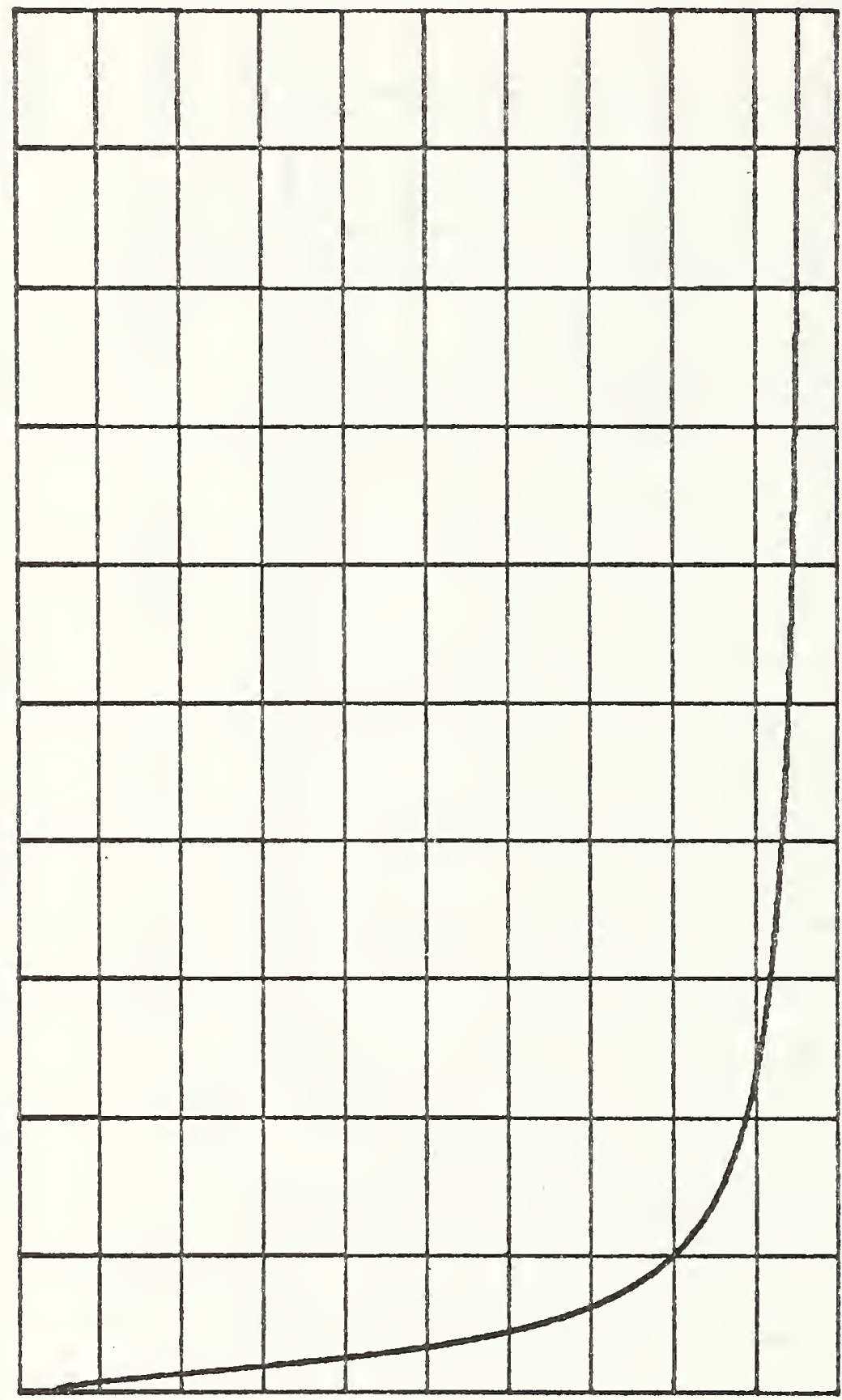

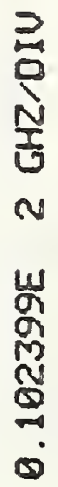

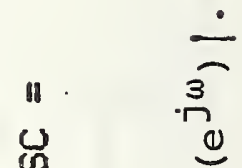

告

웅

वे

II

(1)

皆 


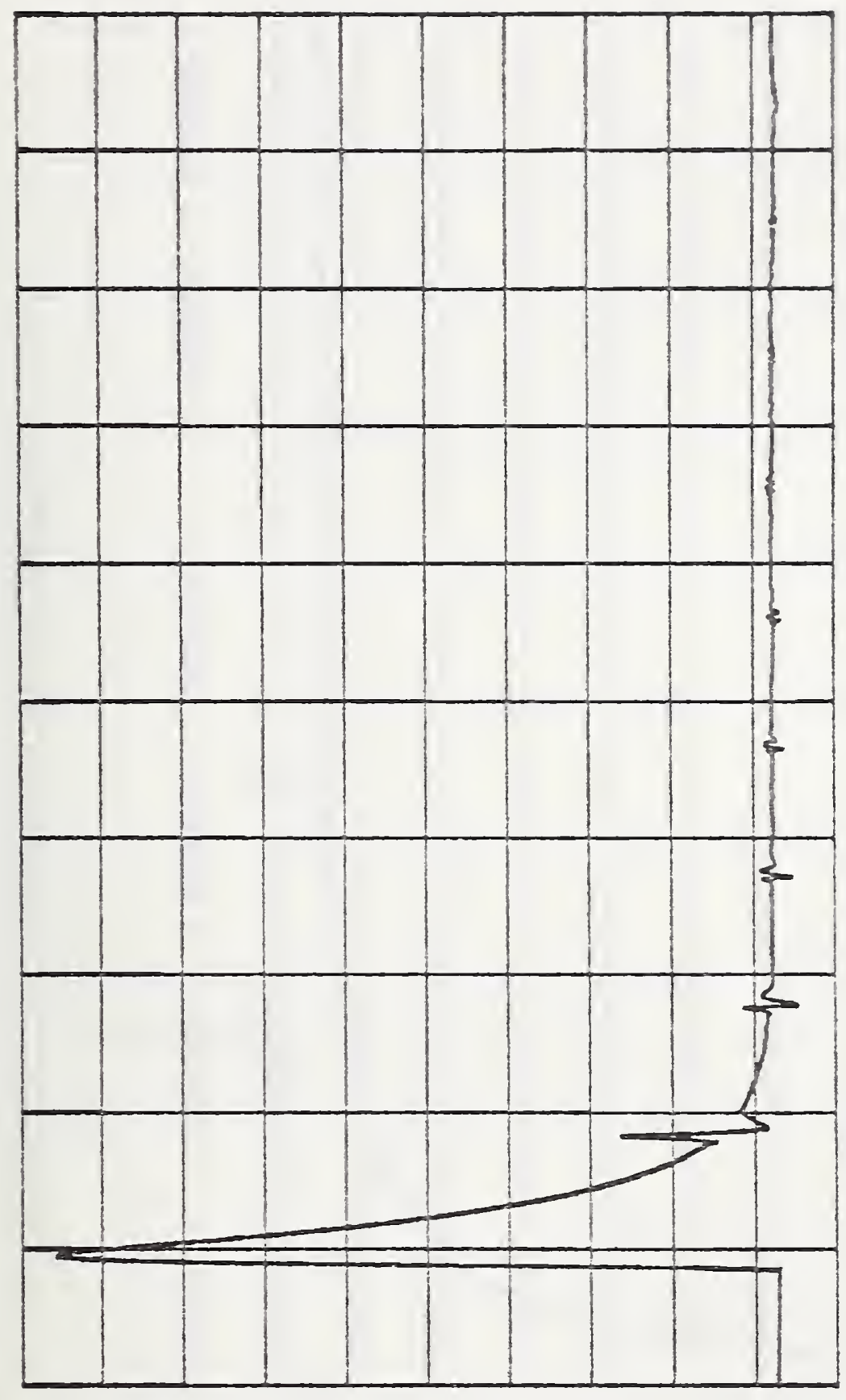

$\frac{2}{2}$

宸 异

ते

हู

II

옹

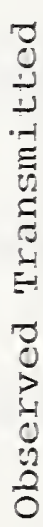

i

w

II

()

皆 


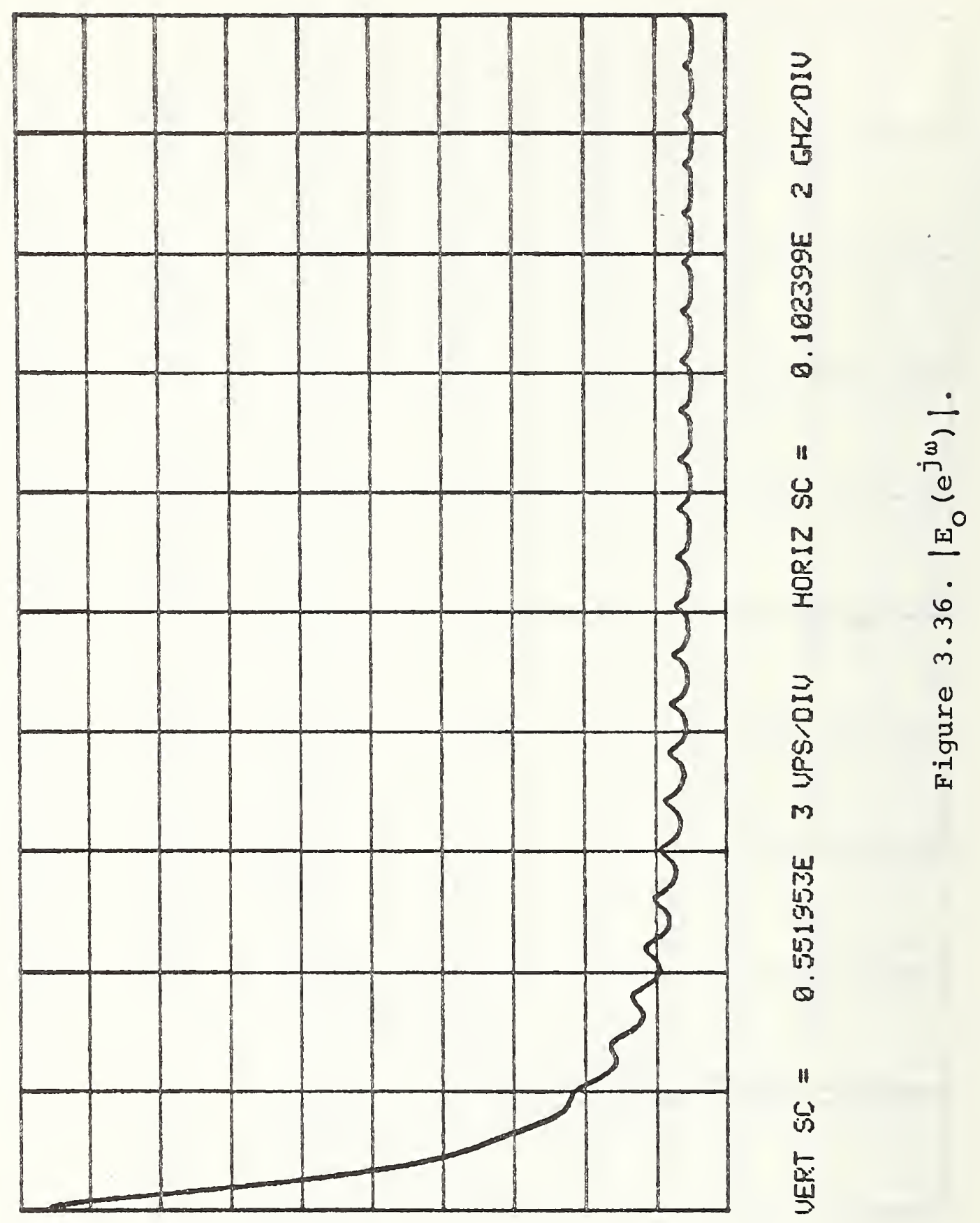




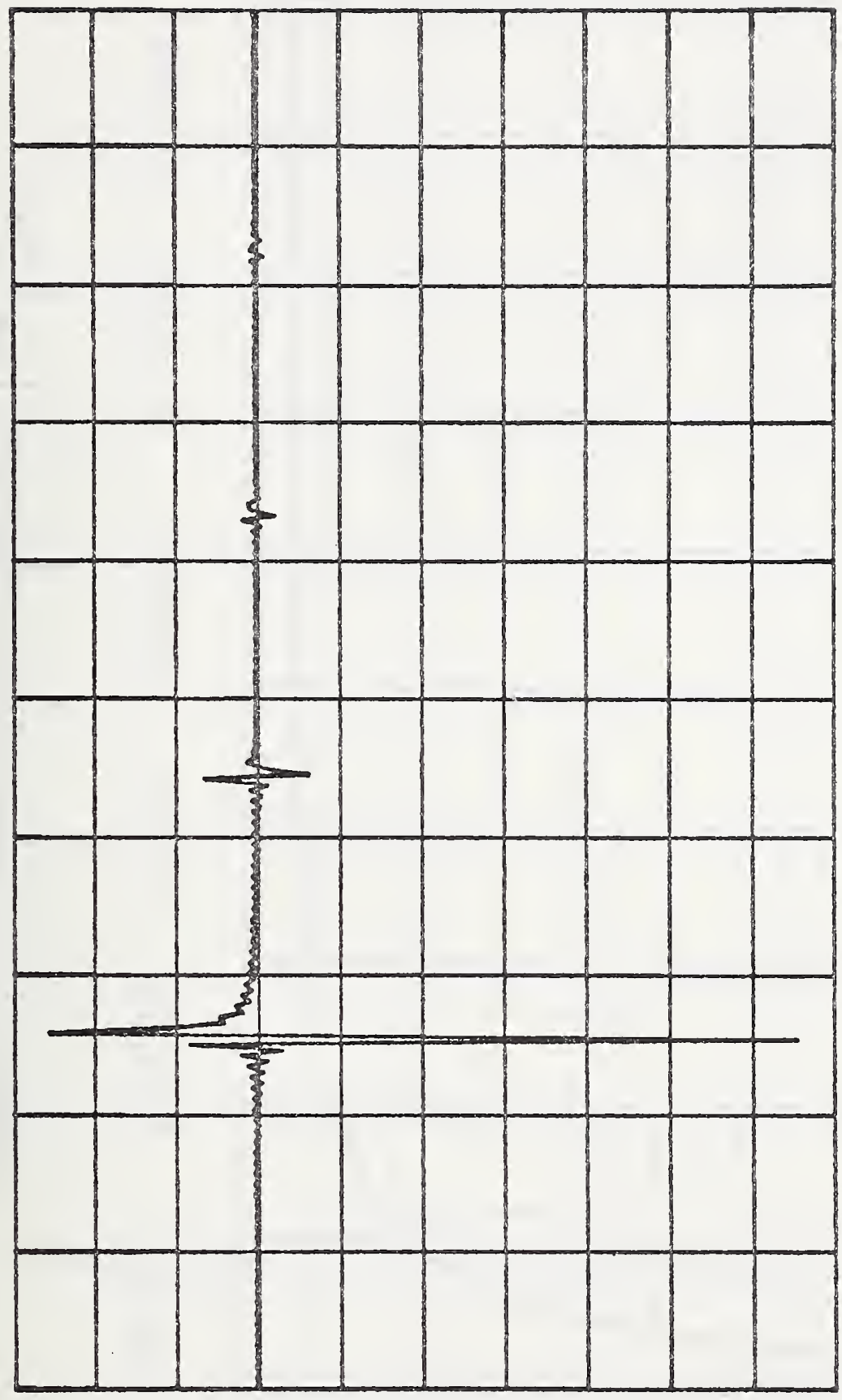

2
0
0
0

4
0
0
0
0

¿

$<0^{\circ}$

0
0
0
0

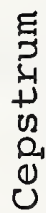

㝴

in

w

M

0

0

II

(5)

$\frac{5}{2}$ 

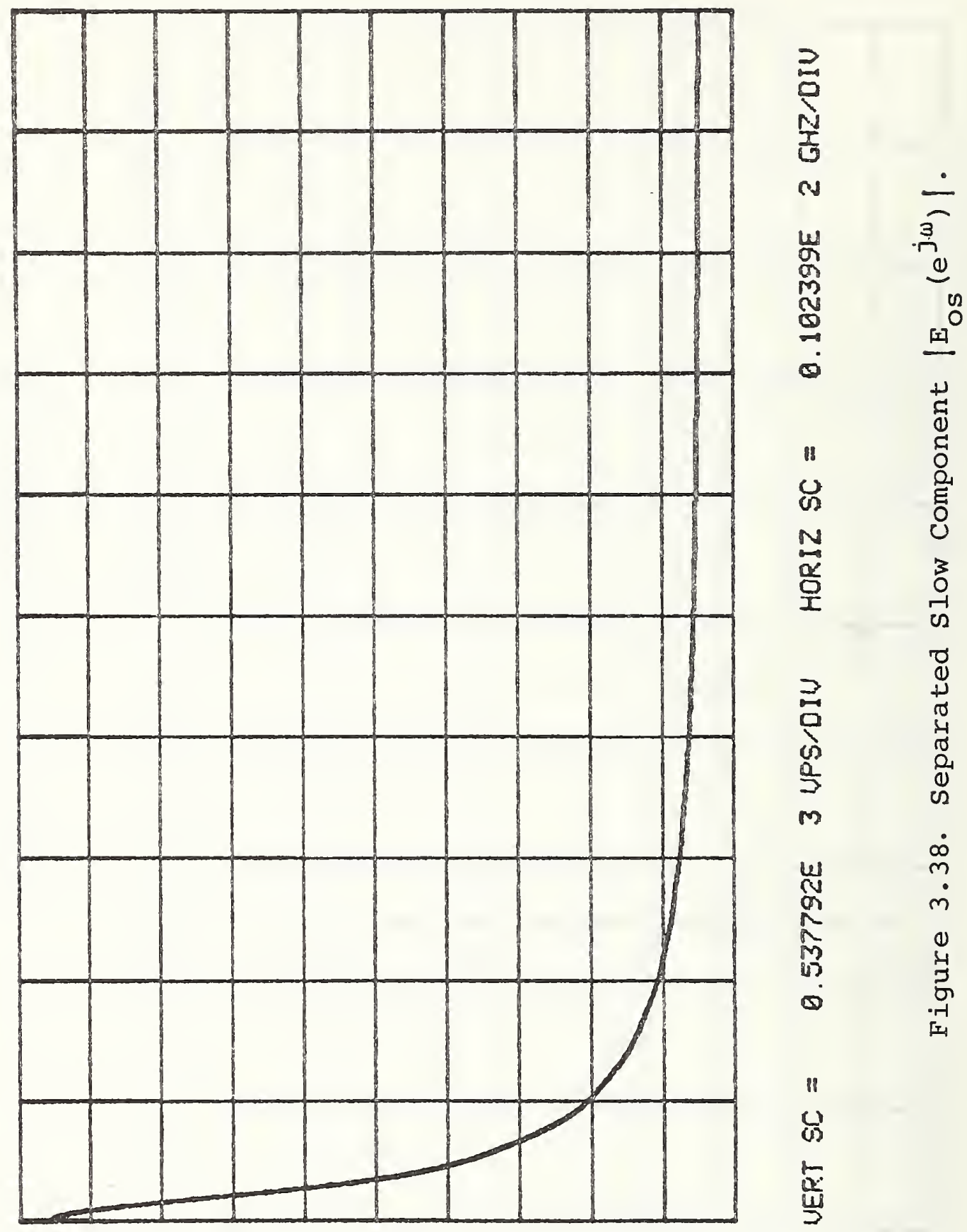


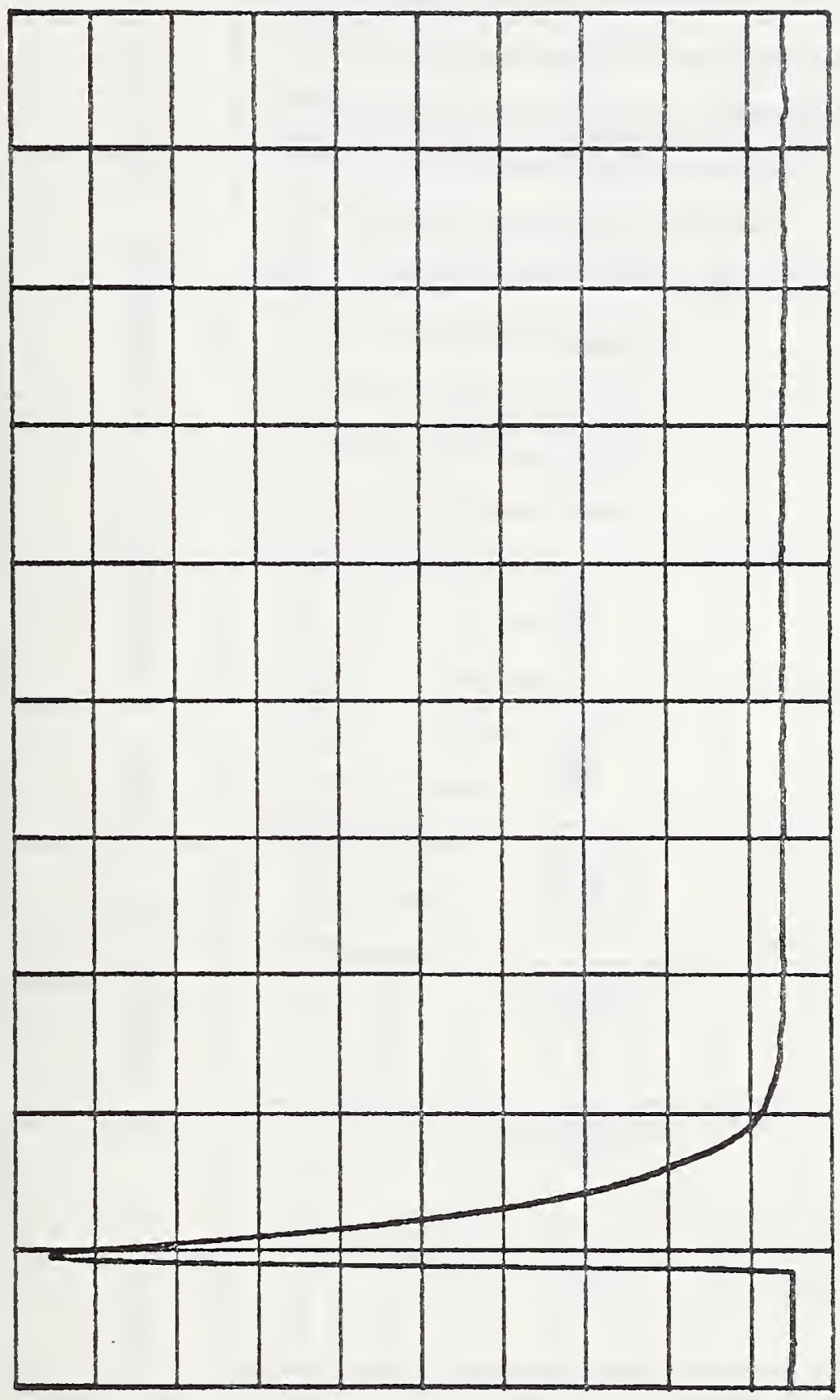

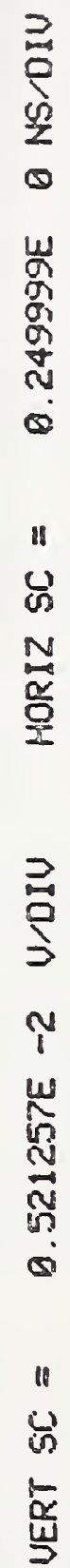

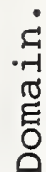

:

.

+1
0
0
0
0
0
0
0
0

3

$\begin{array}{ll}2 & 0 \\ 0 & 0 \\ 1 & 0 \\ 1 & 0 \\ 1 & 0 \\ 0 & 0 \\ 0 & 0\end{array}$

กิ 


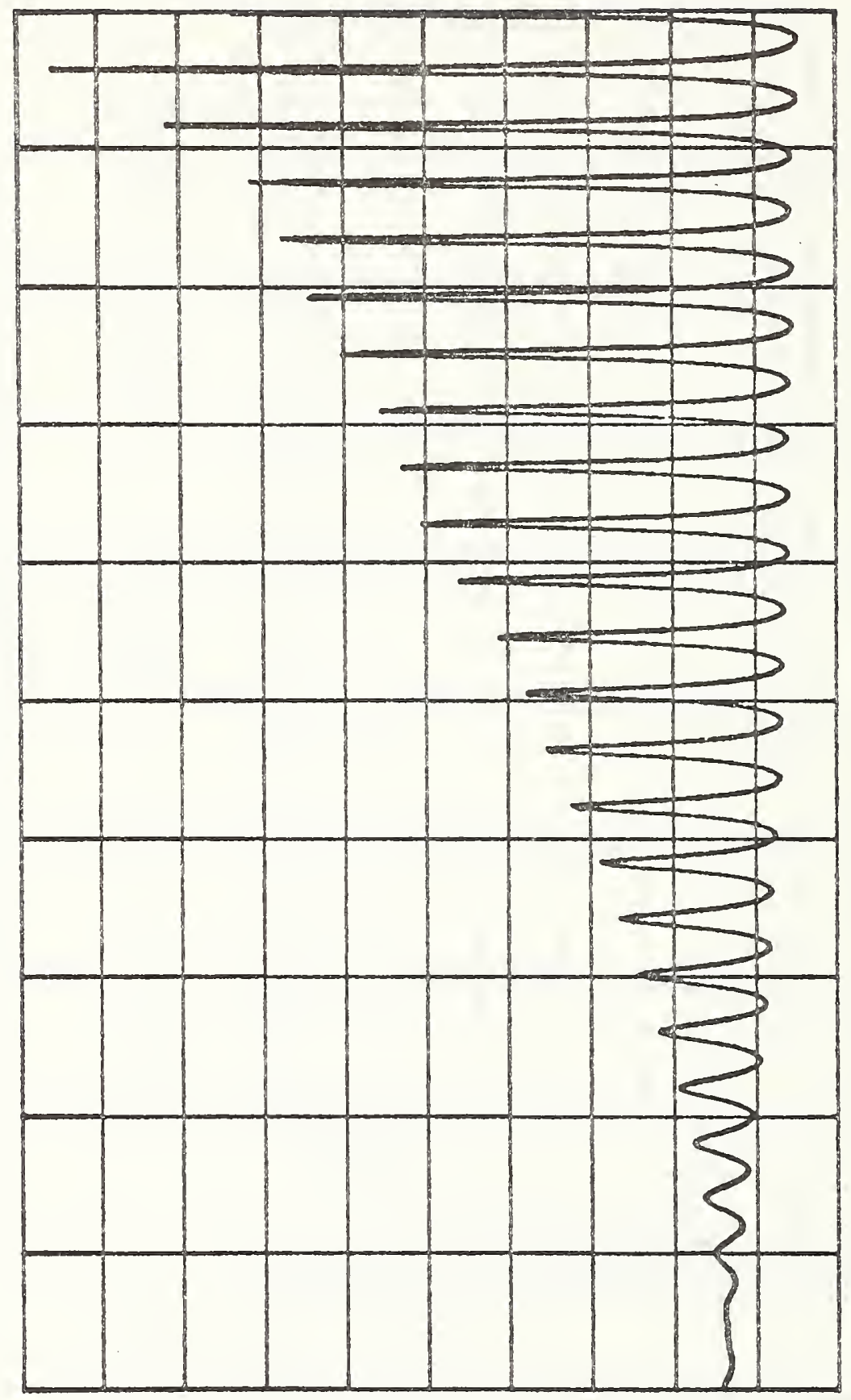

$\frac{2}{1}$

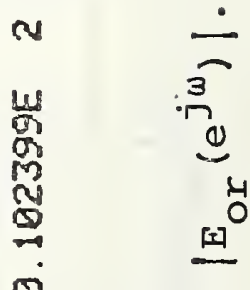

$\infty$

U
N
음

¿્ટ

r.

2

0
0
0
0
0
0
0
0

$\sim$

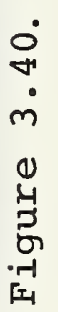

18

ज)

岕 


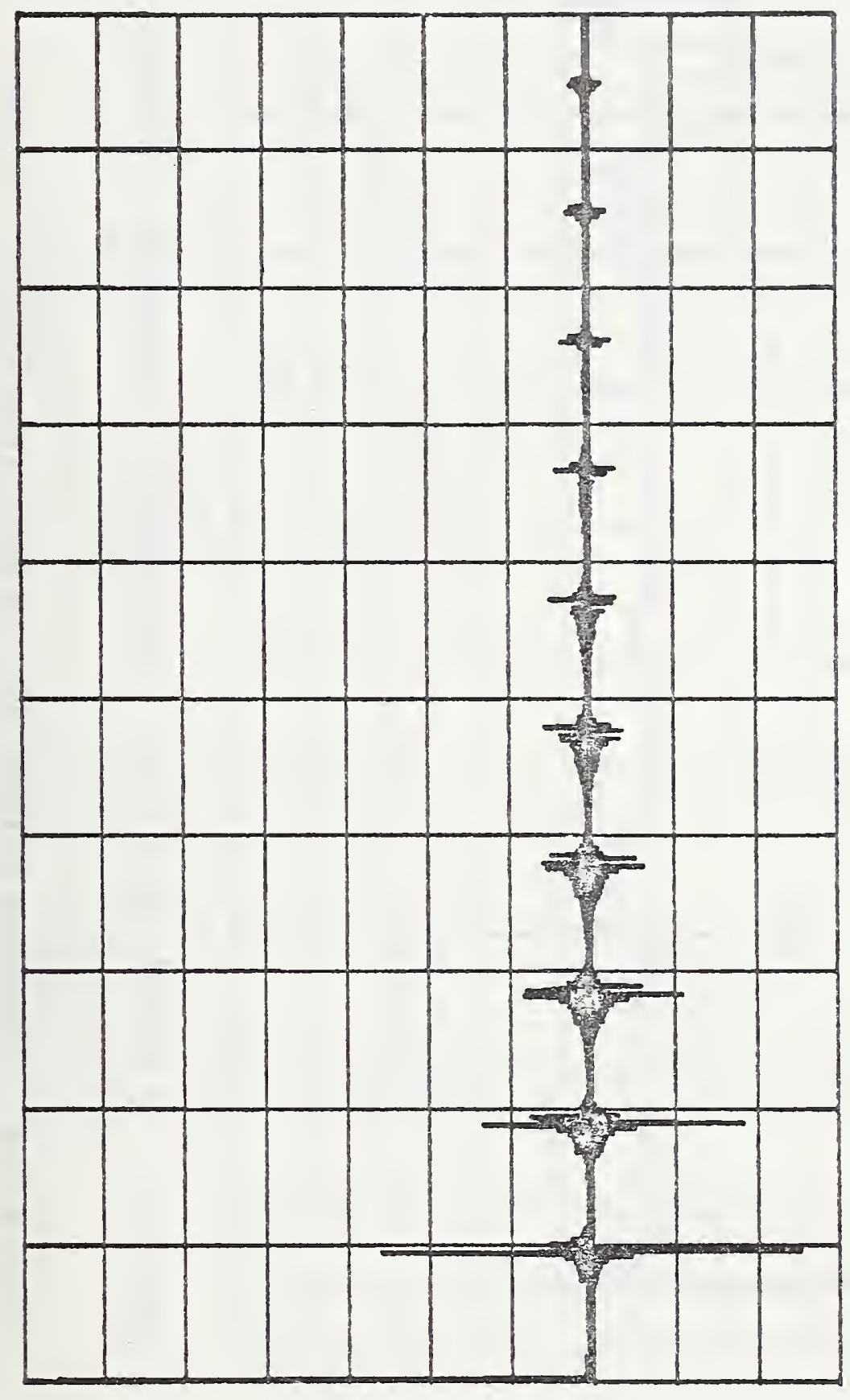

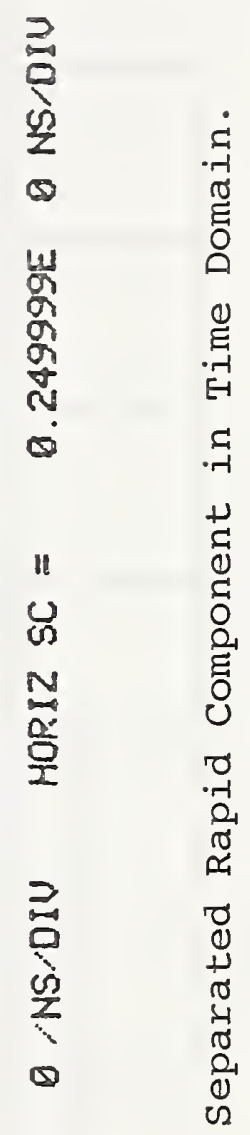

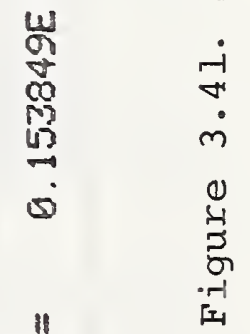

ơ

占 


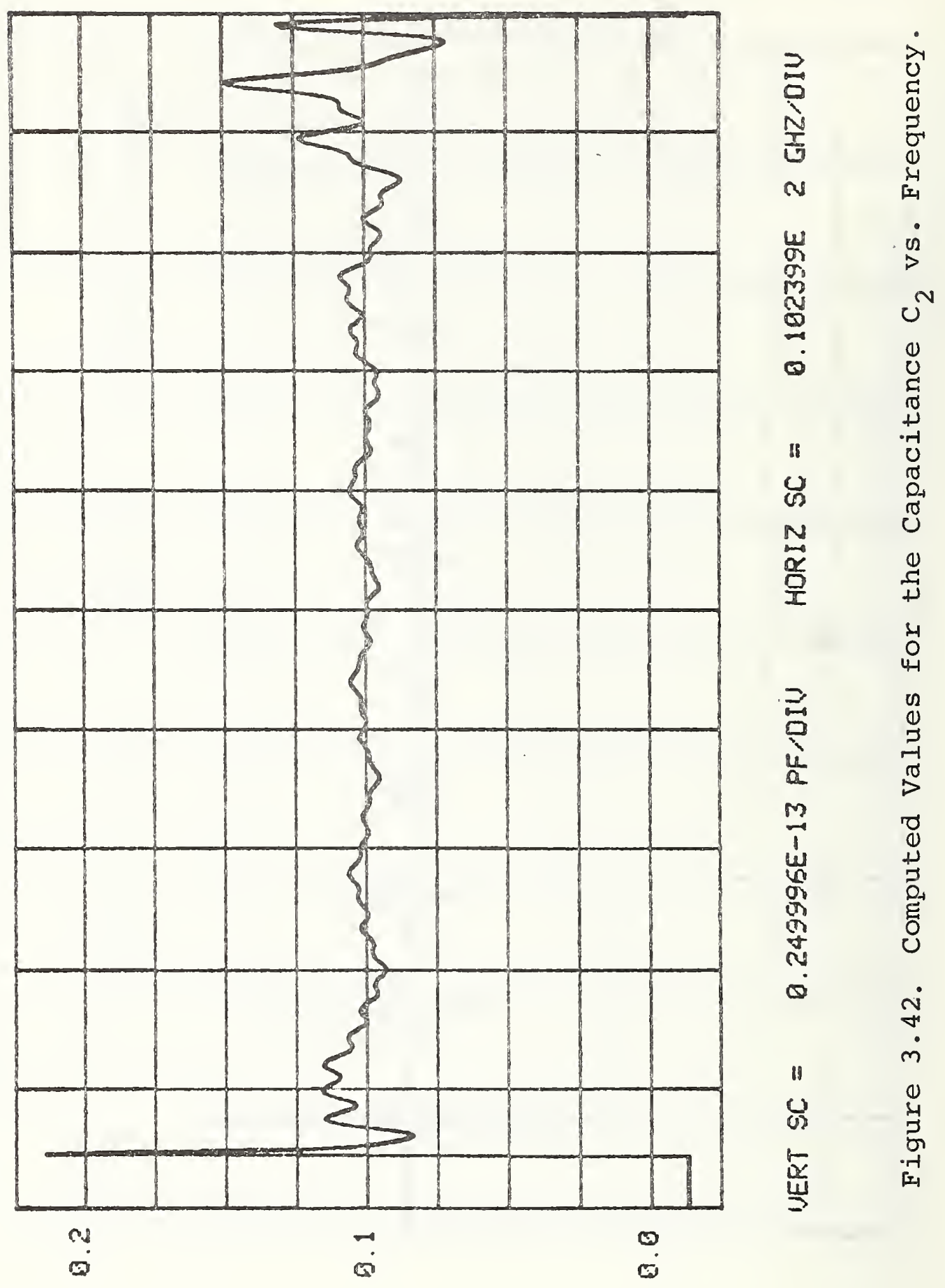



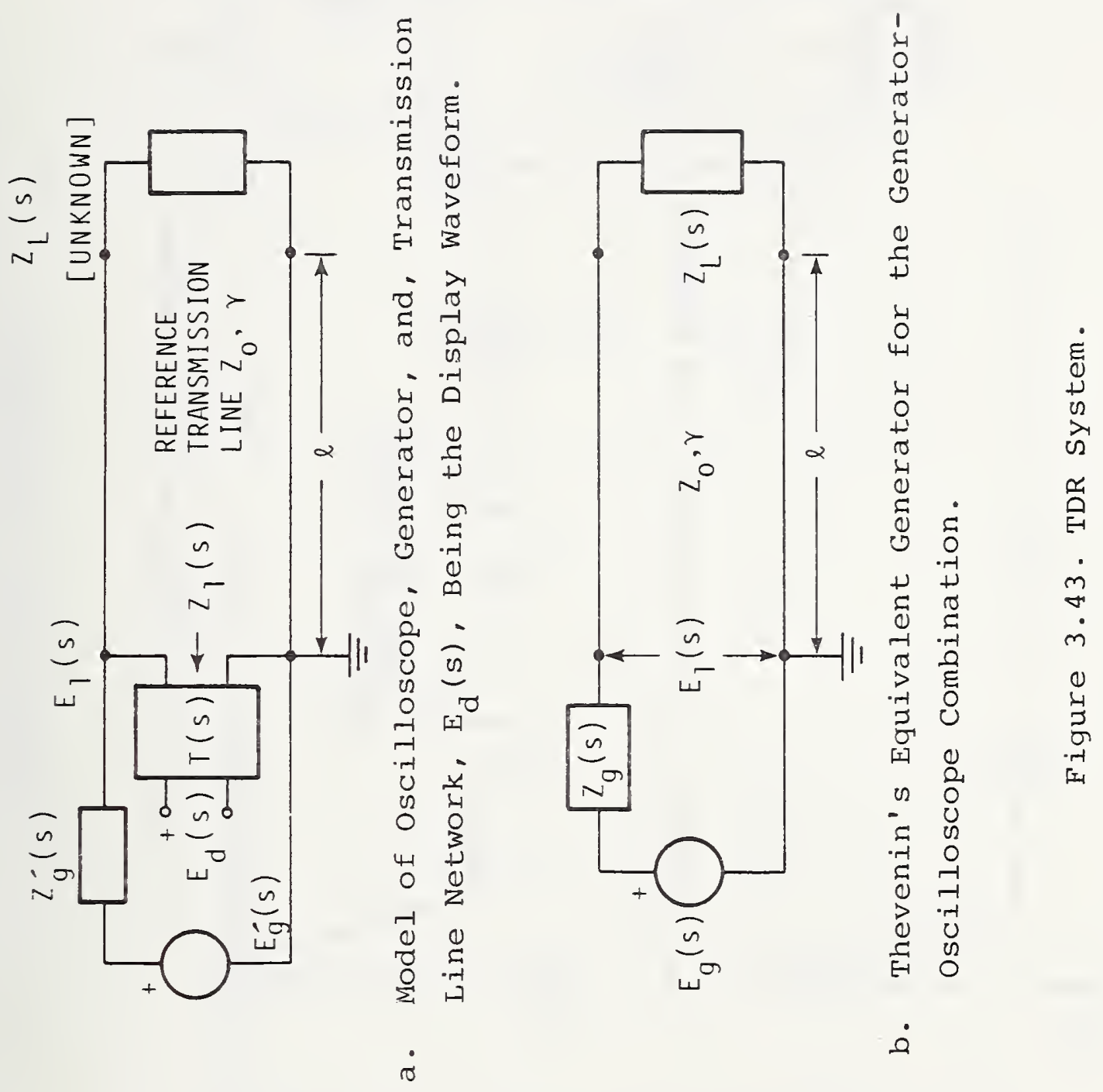

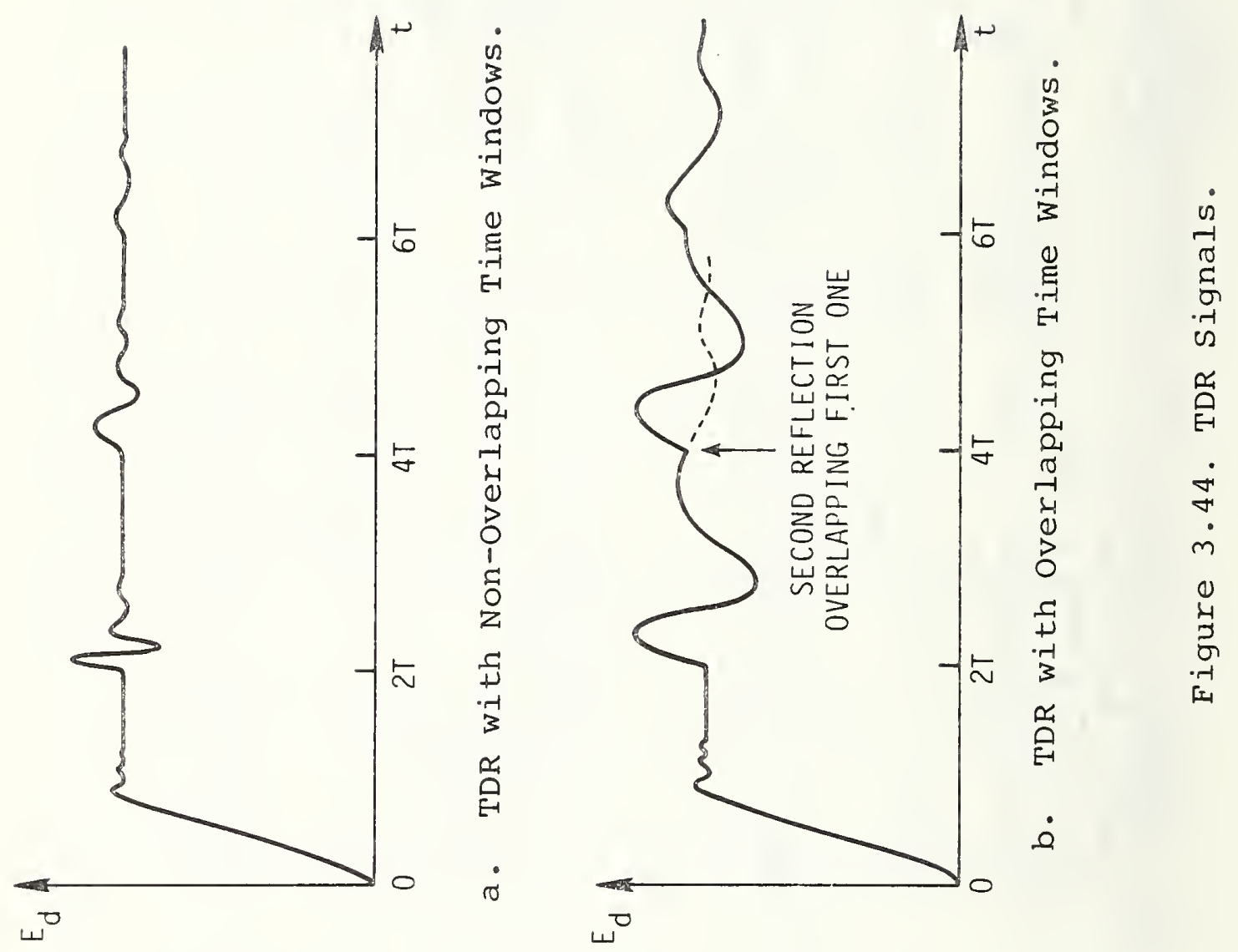


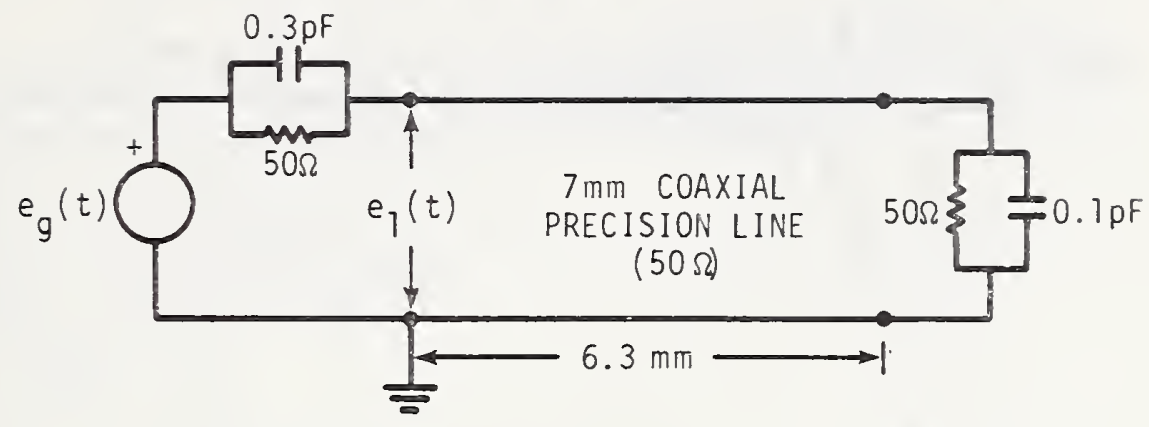

Figure 3.45 Network.

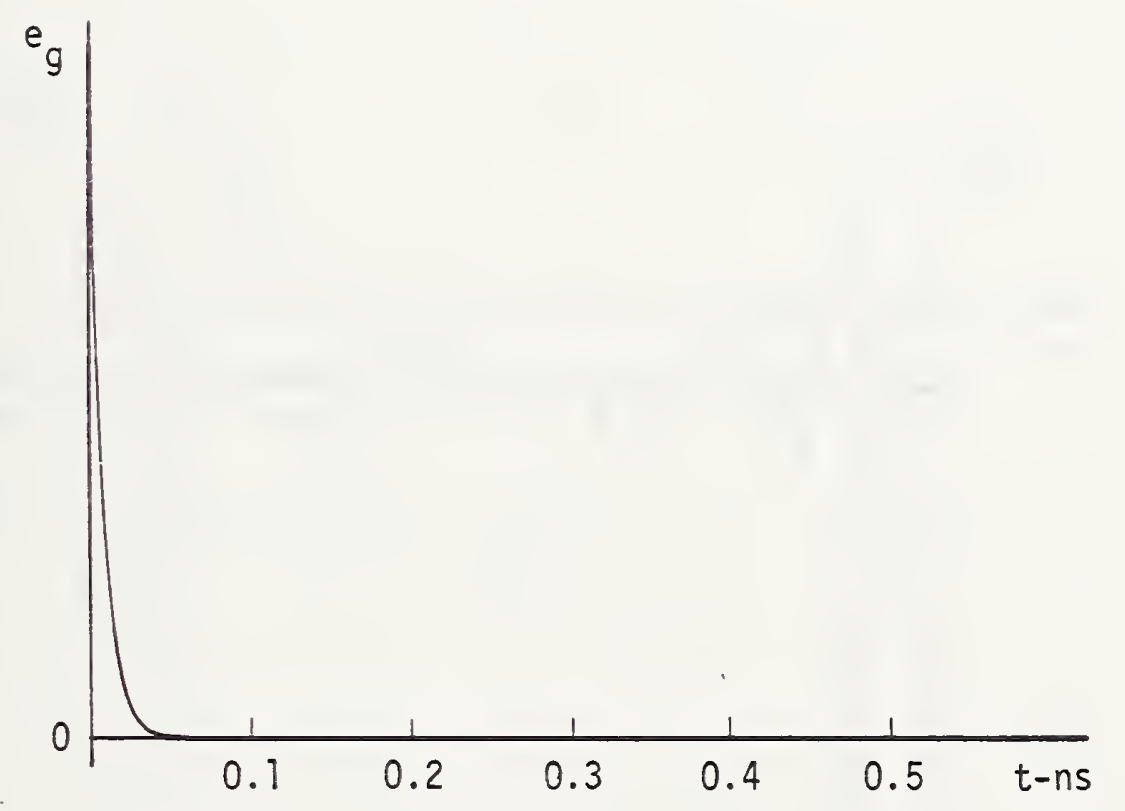

Figure 3.46. $\mathrm{e}_{\mathrm{g}}(\mathrm{t})=\mathrm{Ae}-\mathrm{t/ \tau} ; \tau=7.96 \mathrm{ps}$. 


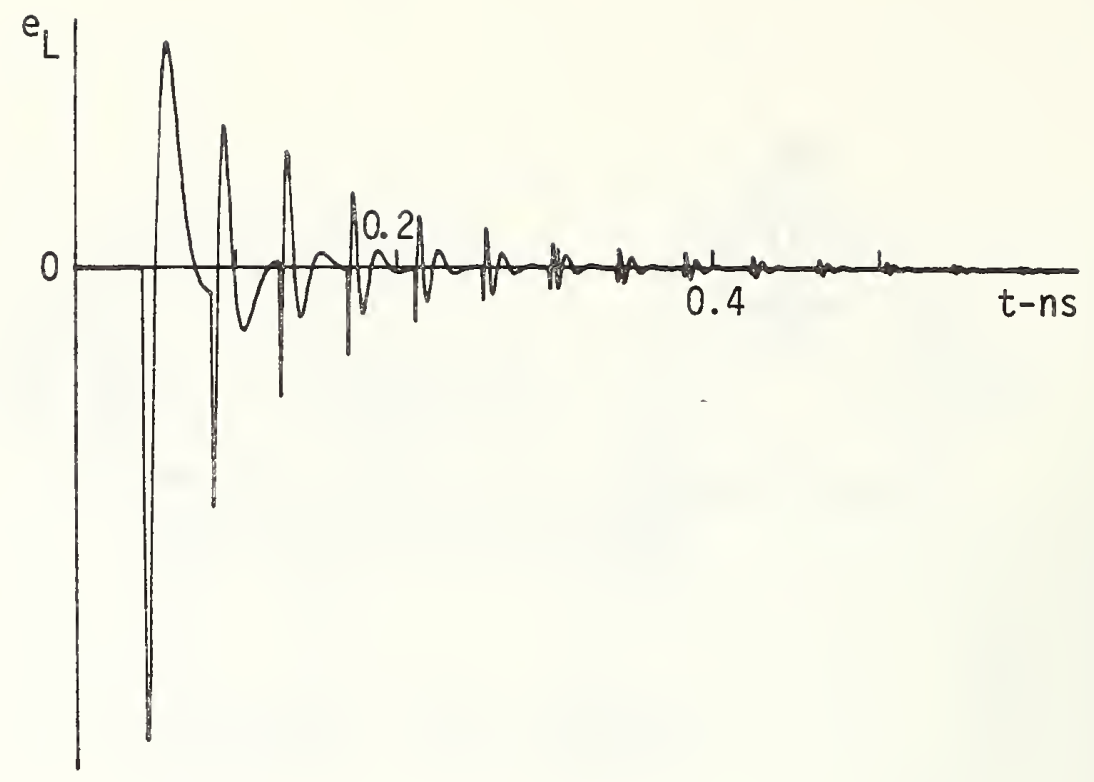

Figure 3.47. $e_{L}(t)=e_{d L}(t)-e_{d M}(t)$.

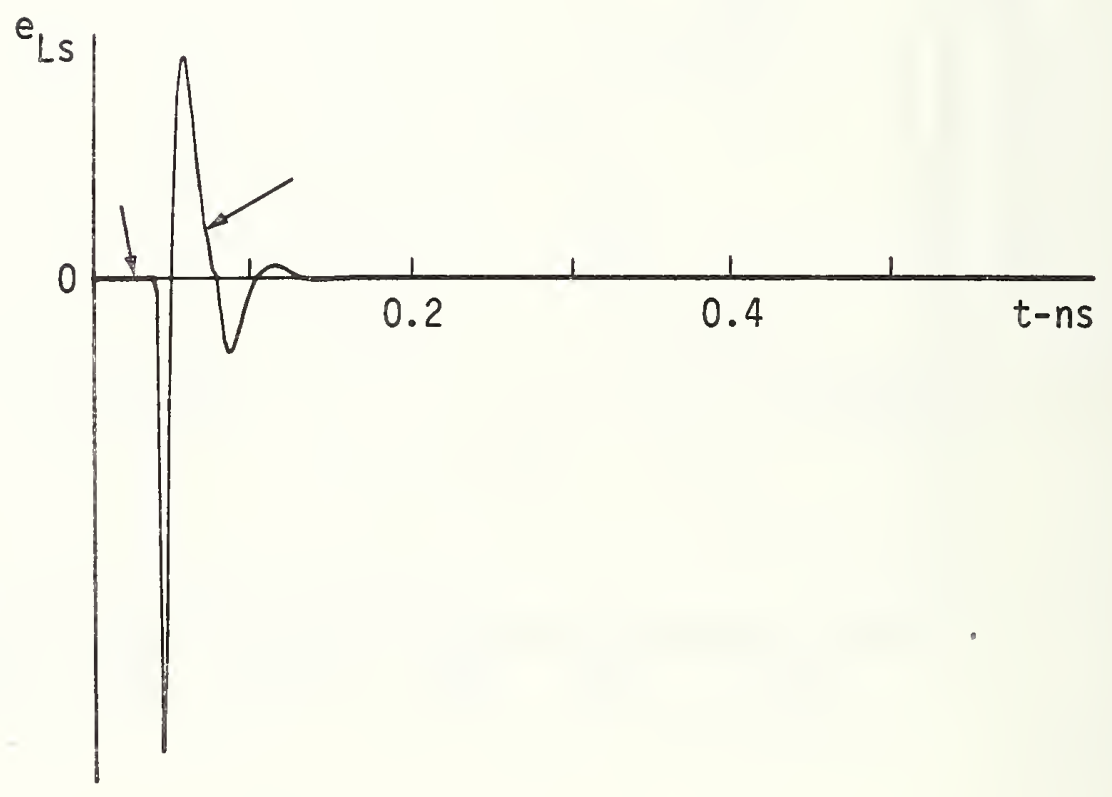

Figure 3.48. $e_{L s}(t)$, Arrows Indicate Error Oscillations.

$-76-$ 


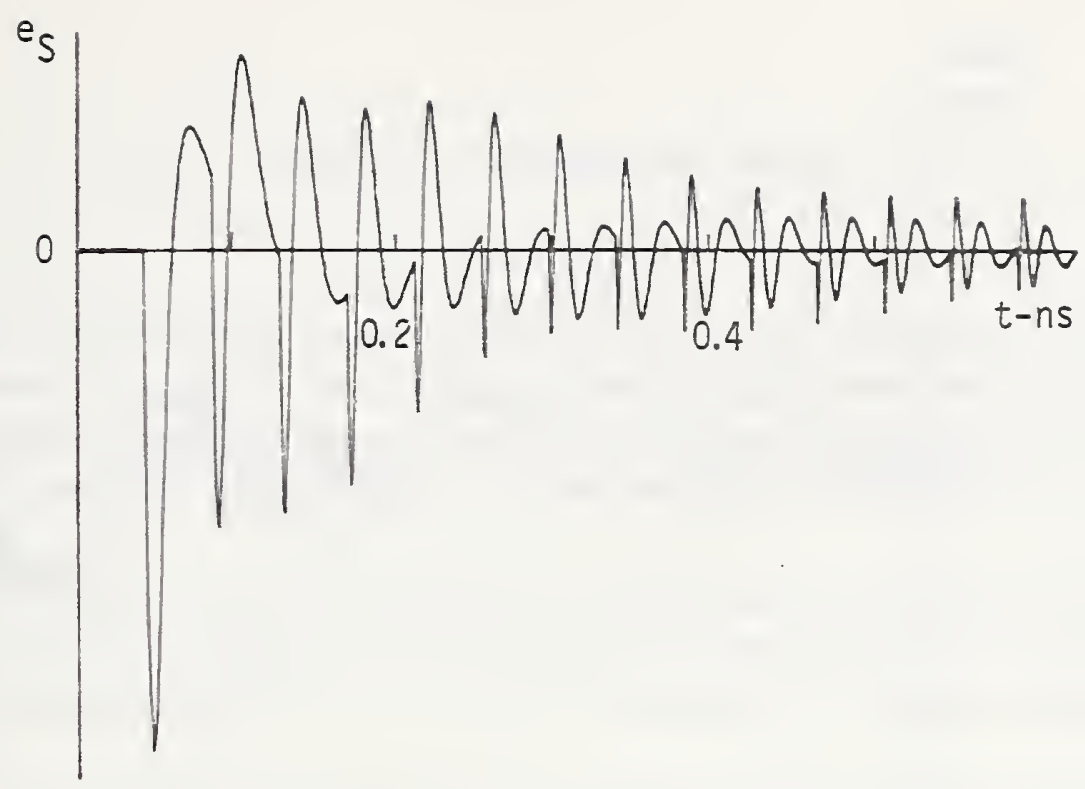

Figure 3.49. $e_{S}(t)=e_{\mathrm{dS}}(t)-e_{\mathrm{dM}}(t)$.

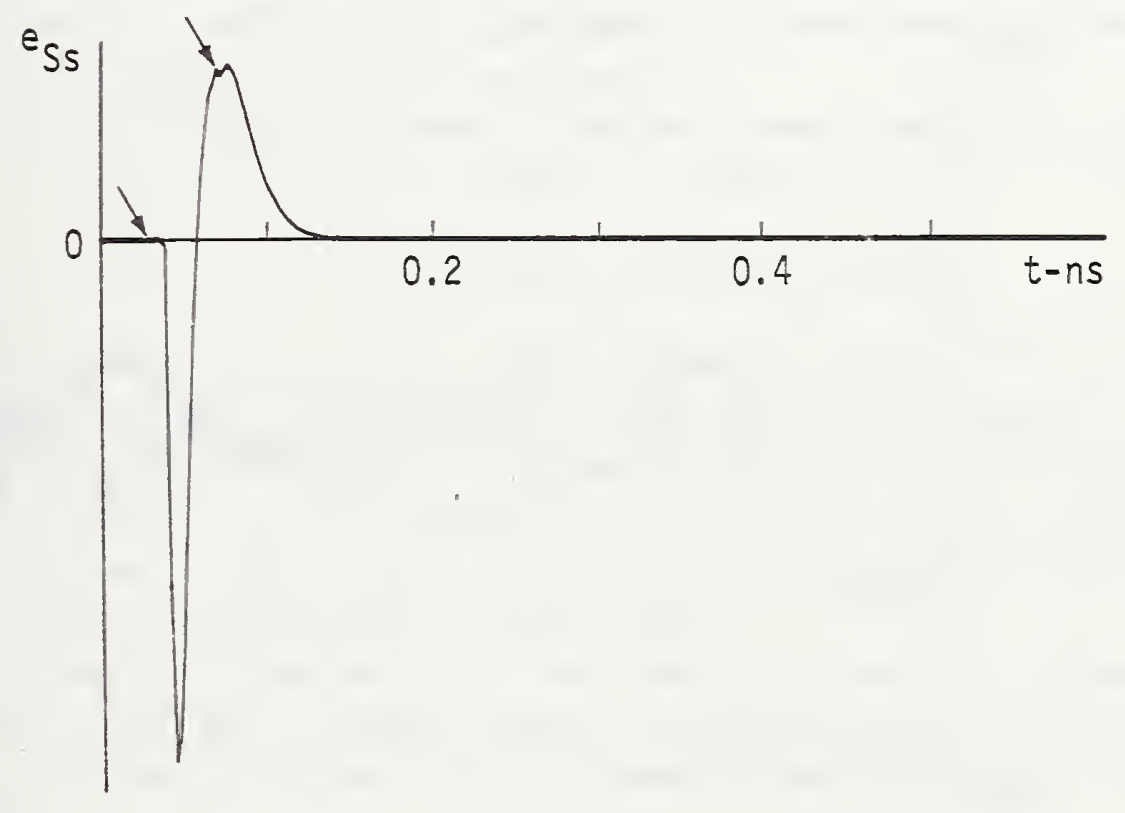

Figure 3.50. e Ss $(t)$, Arrows Indicate Error Oscillations. 


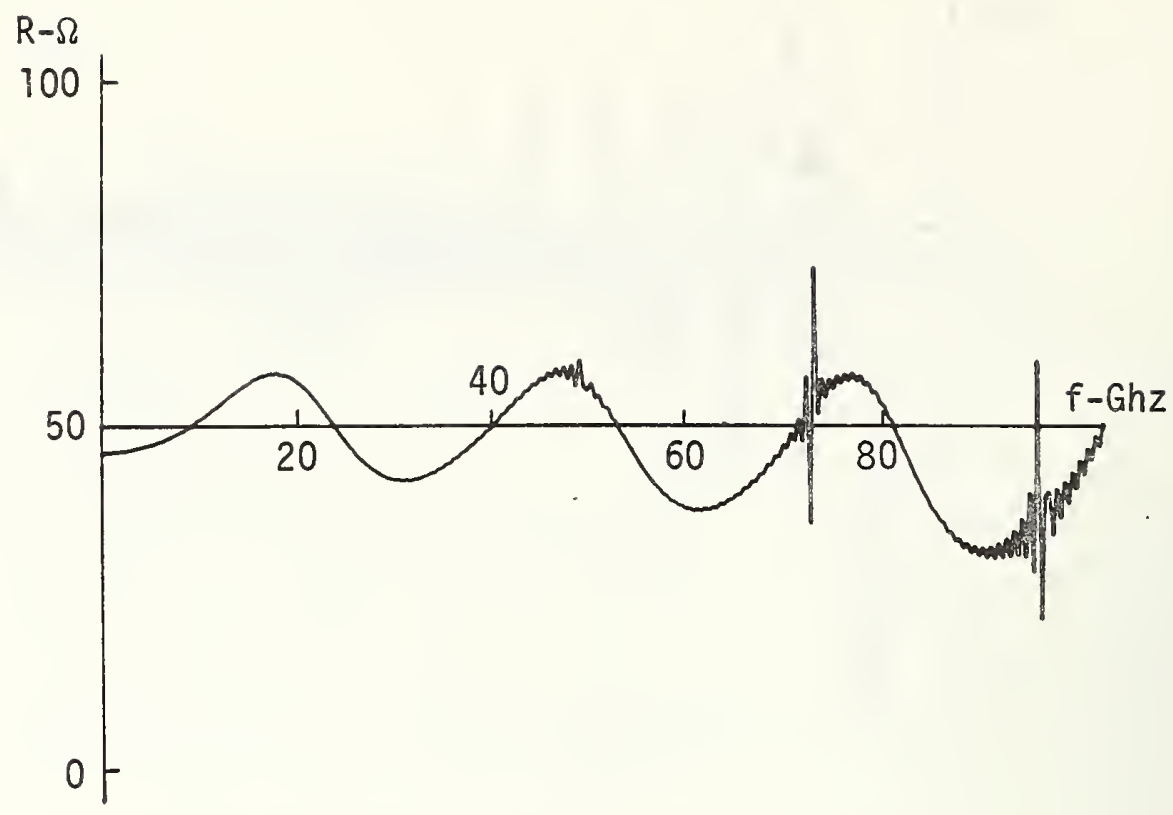

Figure 3.51. Load Resistance.

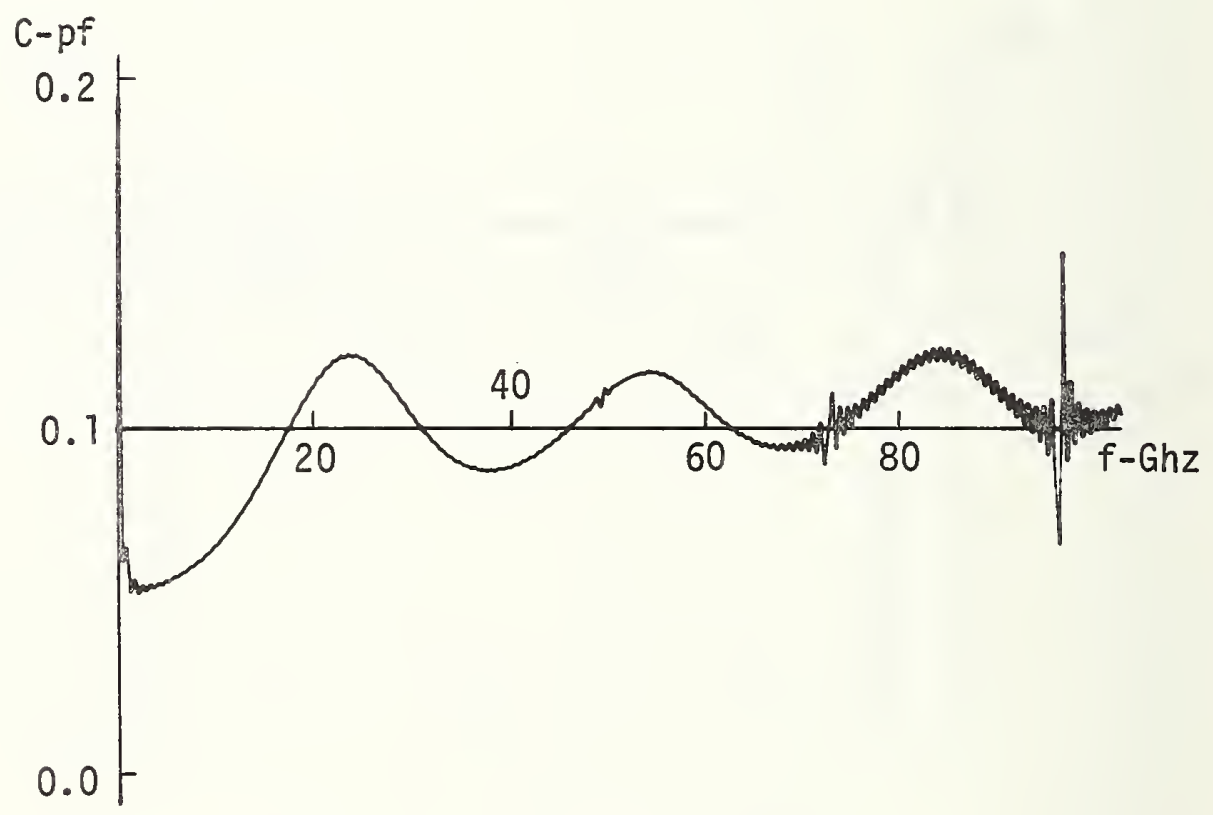

Figure 3.52. Load Capacitance. 


\section{TIME DOMAIN DIELECTRIC SPECTROSCOPY}

\subsection{Introduction}

In chapter 3 the homomorphic deconvolution transform was successful in solving various time domain network analysis problems, and more specifically, time domain reflectometry and transmission problems. Network parameters of interest were identified, and more importantly, the time domain overlap of multiple reflections did not put any limitations or restrictions on the method.

In the present chapter another application of time domain measurements will be considered. Here, the homomorphic deconvolution transform will be applied to time domain dielectric spectroscopy (TDDS); i.e., the study of dielectric relaxation phenomenon by time domain methods.

TDDS is in fact a TDR problem, but with the emphasis being turned to the dielectric material filling the transmission line in order to evaluate the frequency dependent complex dielectric constant (relative permittivity) $k(\omega)$.

As was indicated in chapter 3, the conventional TDR method is based upon and limited by time windowing to select the relevant or desired waveform epoch. Thus, for the conventional TDR method to be used, the dielectric sample has to be either relatively infinitesimal or relatively infinite in length to obtain nonoverlapping time windows. The homomorphic deconvolution method will be applied in the present chapter to remove these restrictions and allow the use of finite size samples.

The method will be applied to a model typical of dielectric materials having polarization absorptions over the frequency range from $1 \mathrm{kHz}$ upward to $100 \mathrm{GHz}$.

\subsection{Theory of Time Domain Dielectric Spectroscopy}

The typical experimental setup for a TDDS measurement [12-18] is shown in figure 4.1. The dielectric material to be characterized is formed into the shape required to fill a coaxial sample holder of finite length d corresponding to an empty precision line having a characteristic impedance $z_{0}(\omega)$ and a propagation constant $\gamma(\omega)$. A time domain waveform $e_{1}(t)$ is to be recorded. This waveform consists of the multiple reflections from both dielectric-air interfaces as well as the incident wave.

Because the physical dimensions of the two transmission lines are the same, the characteristic impedance $z_{d}(\omega)$ and the complex propagation constant $\gamma_{d}(\omega)$ of the dielectric filled transmission line are related to the corresponding quantities of the uncelled air line as follows:

$$
z_{d}(\omega)=z_{0}(\omega) / \sqrt{k(\omega)}
$$

and

$$
\gamma_{d}(\omega)=\gamma_{0}(\omega) \cdot \sqrt{k(\omega)}
$$


where $k(\omega)$ is the complex dielectric constant of the sample. The reflection coefficient at the air-dielectric interface on the line $\rho(\omega)$ is given by

$$
\rho(\omega)=\left[Z_{d}(\omega)-Z_{o}(\omega)\right] /\left[Z_{d}(\omega)+Z_{o}(\omega)\right]
$$

which upon substituting eq. (4.1) takes the form

$$
\rho(\omega)=[1-\sqrt{k(\omega)}] /[1+\sqrt{k(\omega)}] .
$$

The form of eq. (4.4) can be solved for $k(\omega)$ as follows:

$$
\sqrt{\mathrm{k}(\omega)}=(1-\rho(\omega)) /(1+\rho(\omega))
$$

or

$$
k(\omega)=[(1-\rho(\omega)) /(1+\rho(\omega))]^{2} .
$$

This equation, eq. (4.5), summarizes the concept of using the TDR method in dielectric characteristics by experimentally observing the reflected wave produced by the reflection coefficient $\rho(\omega)$. The deduced reflection coefficient $\rho(\omega)$ is then substituted in eq. (4.5) to yield the complex dielectric constant $k(\omega)$.

In the conventional TDDS method [12-18] the first reflection coming back from the airdielectric interface determines the measurement's time window; i.e., the reflected waveform is observed for the time interval containing the first reflection. This first reflection has a Fourier transform which is the product of the Fourier transforms of the incident signal $e_{g}(t) / 2$ and the reflection coefficient. The separability of the time window and the knowledge of $e_{g}(t)$ allow the calibration of $\rho(\omega)$ and ultimately $k(\omega)$.

As mentioned in the introduction section, the separability of the first reflection requires either an infinitesimal or an infinite sample length. The requirement of having a very long sample is not generally practical. On the other hand an infinitesimal sample would not alter $e_{g}(t)$ to any great degree, thereby not imparting to the signal waveform much information about the dielectric. A finite sample of practical size will produce overlapping multiple reflections thus invalidating the conventional (windowing) method. In such cases, the homomorphic deconvolution transform will succeed in removing (deconvoluting) the effects of multiple reflections allowing the determination of $\rho(\omega)$.

The frequency domain form $E_{1}\left(e^{j \omega}\right)$ of the recorded waveform $e_{1}(t)$ is given by

$$
E_{1}\left(e^{j \omega}\right)=\frac{E_{g}\left(e^{j \omega}\right)}{2} \rho(\omega) \exp \left(-2 \gamma_{0}(\omega) \ell\right) \cdot \frac{1-\exp \left(-2 \gamma_{d}(\omega) d\right)}{1-\rho^{2}(\omega) \exp \left(-2 \gamma_{d}(\omega) d\right)} \text {. }
$$

By virtue of eqs. (4.2) and (4.4), the above eq. (4.6) has only one unknown $k(\omega)$ provided that $E_{g}\left(e^{j \omega}\right), Z_{o}(\omega)$, and $\gamma_{0}(\omega)$ are known. However, such an equation can not be solved, as it is, to yield this unknown. Some research workers [12] have gone through approximations to simplify this equation; but the use of the homomorphic deconvolution transform on eq. (4.6), as it is, will yield the required information needless of any assumptions or approximations. 
4.3 The Homomorphic Deconvolution as Applied to TDDS Problems

The homomorphic deconvolution transform that was first developed in chapter 2, and then used with TDR networks in chapter 3, separates a convolution into its two components. These two components are the slowly and rapidly varying frequency domain components. By examining eq. (4.6) the slowly and rapidly varying components can be identified as

$$
E_{1 s}\left(e^{j \omega}\right)=\frac{E_{g}\left(e^{j \omega}\right)}{2} \exp \left(-2 \gamma_{0}(\omega) \ell\right) \rho(\omega),
$$

and

$$
E_{1 r}\left(e^{j \omega}\right)=\frac{1-\exp \left(-2 \gamma_{d}(\omega) d\right)}{1-\rho^{2}(\omega) \exp \left(-2 \gamma_{d}(\omega) d\right)}
$$

respectively. In these two equations, eq. (4.7) is a simple one that can be solved in $\rho(\omega)$, and consequently $k(\omega)$, while eq. (4.8) still has the same complications that originally existed in eq. $(4.6)$.

\subsection{Model for Debye Dielectric $[9,11]$}

One well known class of dielectric materials will be considered in the application presented in this chapter, the single relaxation time dielectric known as the Debye dielectric. The complex dielectric constant $k(\omega)$ of a Debye dielectric is given by

$$
k(\omega)=k_{\infty}+\frac{k_{0}-k_{\infty}}{1+j \omega \tau}
$$

where $k_{0}=k(\omega=0), k_{\infty}=k(\omega=\infty)$, and $\tau$ is the dielectric relaxation time. This equation is commonly put in the form

$$
k(\omega)=k^{\prime}(\omega)-j k^{\prime \prime}(\omega)
$$

where

$$
k^{\prime}(\omega)=\left(k_{0}+\omega^{2} \tau^{2} k_{\infty}\right) /\left(1+\omega^{2} \tau^{2}\right)
$$

and

$$
k^{\prime \prime}(\omega)=\omega \tau\left(k_{0}-k_{\infty}\right) /\left(1+\omega^{2} \tau^{2}\right) .
$$

The plot $k^{\prime}(\omega)$ vs. $k^{\prime}(\omega)$ is a semicircle of radius $r$ and centered at the point $(c, 0)$ where

$$
r=\left(k_{0}-k_{\infty}\right) / 2
$$

and

$$
c=\left(k_{0}+k_{\infty}\right) / 2
$$

the point $(c, r)$ is the point at which $\omega=\omega_{0}$, where

$$
\omega_{0} \tau=1 \text {. }
$$


The semicircle is shown in figure 4.2; this semicircle is commonly called the Debye semicircle or the Cole-Cole plot [10-11].

It is important to mention that the complex dielectric constant parameters $k_{0}, k_{\infty}$, and $\tau$ can be determined once the dielectric semicircle is obtained. This is achieved by virtue of the eqs. $(4.13),(4.14)$, and $(4.15)$ to be

$$
\begin{aligned}
& k_{0}=c+r, \\
& k_{\infty}=c-r,
\end{aligned}
$$

and

$$
\tau=1 / \omega_{0} \cdot
$$

Also the circular geometry of the graph provides a means of consistently smoothing experimental data for $k^{\prime}(\omega)$ and $k^{\prime \prime}(\omega)$.

Finally, the point should be made that the TDDS methods developed here and not restricted to any particular dielectric model. The Debye model has been chosen because it is a well known model for the dilute mixture of polar molecules in nonpolar solvents. As such the model will clearly demonstrate the utility of the deconvolution method in obtaining the dielectric parameters, $k^{\prime}(\omega)$ and $k^{\prime \prime}(\omega)$.

In general, a dielectric material may contain a distribution of relaxation times comprising its relaxation process. The relative dielectric constant may be viewed as a sum of relaxation processes each having a distribution of relaxation times [19].

$$
k(\omega)=k_{\infty}+\int_{0}^{\infty} \frac{g(\tau)}{1+j \omega \tau} d \tau
$$

where $g(\tau)$ is the relaxation time distribution function.

\subsection{Application of Homomorphic Deconvolution in TDDS [20]}

A computer program was written to simulate the TDDS setup of figure 4.1. The transmission line characteristic impedance was $50 \Omega$ and the dielectric sample length was one cm. The dielectric parameters were chosen to by typical of actual dielectrics satisfying the Debye model. The relative dielectric constant employed was

$$
k(\omega)=4.8+\frac{24.5-4.8}{1+j 2.0 \times 10^{-12} \omega}
$$

in which $k_{0}=24.5, k_{\infty}=4.8$, and $\tau=2 \mathrm{ps}$. The time domain waveform of the generator $e_{g}(t)$ is the same one used in section 3.4 .2 of chapter 3 ; it is given in figure 3.46 . The homomorphic deconvolution transform was applied to the computed signal $E_{1}\left(e^{j \omega}\right)$ to separate both its slowly and rapidly varying components. The slowly varying component together with eq. (4.7) were used to calculate the reflection coefficient $\rho(\omega)$. The resultant values of $\rho(\omega)$ were substituted in eq. (4.5) to yield the desired $k(\omega)$. Figures 4.4, 4.5 , and 4.6 show the results of the above mentioned steps. Figure 4.4 gives the magnitude of $E_{1}\left(e^{j \omega}\right)$; figure 4.5 gives the magnitude of the separated slowly varying component while 
figure 4.6 shows the Cole-Cole plot of the calculated complex dielectric constant components (Argand diagram). A semicircle was fitted [21] to the computed plot and also is given in the figure. The cemicircle parameters were $r=9.80255, c=14.60045$, and $\omega_{0}=0.50071 \mathrm{x}$ $10^{12} \mathrm{rad} / \mathrm{sec}$.

Upon substituting these values in eqs. (4.16), (4.17), and (4.18), the dielectric parameters are evaluated to be

$$
\begin{aligned}
& k_{0}=24.403, \\
& k_{\infty}=4.7979,
\end{aligned}
$$

and

$$
\tau=1.997 \mathrm{ps},
$$

which are within $0.3 \%$ error of their originally given values.

\subsection{Summary and Conclusions}

In this chapter, the theory of the time domain dielectric spectroscopy measurements was presented. Conventionally this method of measurement works only for non-overlapping reflections; i.e., reflections occurring in separate time domain windows. If the reflections overlap, only approximate solutions may be applied to the frequency domain form of the signal.

By applying the homomorphic deconvolution transformation to the TDDS waveform, a new method was developed which was not limited by overlapping reflections. The new method was applied to the well known Debye dielectric model. The method eliminated the overlapping reflections and determined the dielectric parameters to within $0.3 \%$ error of their mode 1 values.

The new method is a general one and has a great utility for time domain measurements on dielectrics since it does not include any restrictions on the dielectric material parameters or require the assumption of a prior physical model. Furthermore, the new method is not restricted to time domain spectroscopy of only dielectric materials but has utility for time domain spectroscopy in general such as for magnetic and other phenomena. 


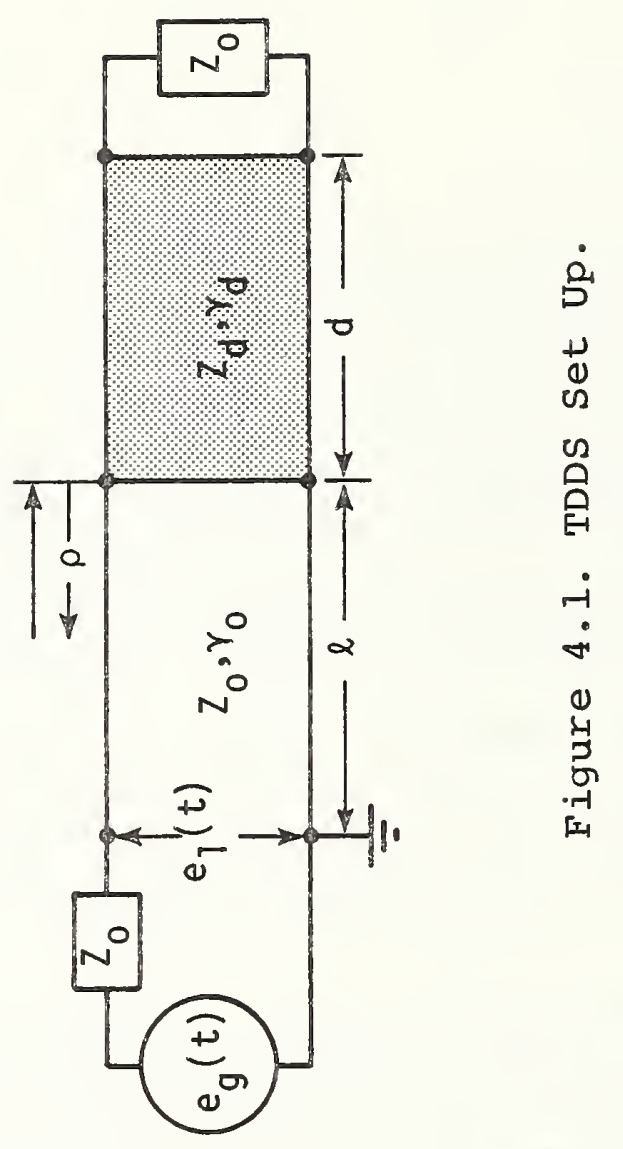




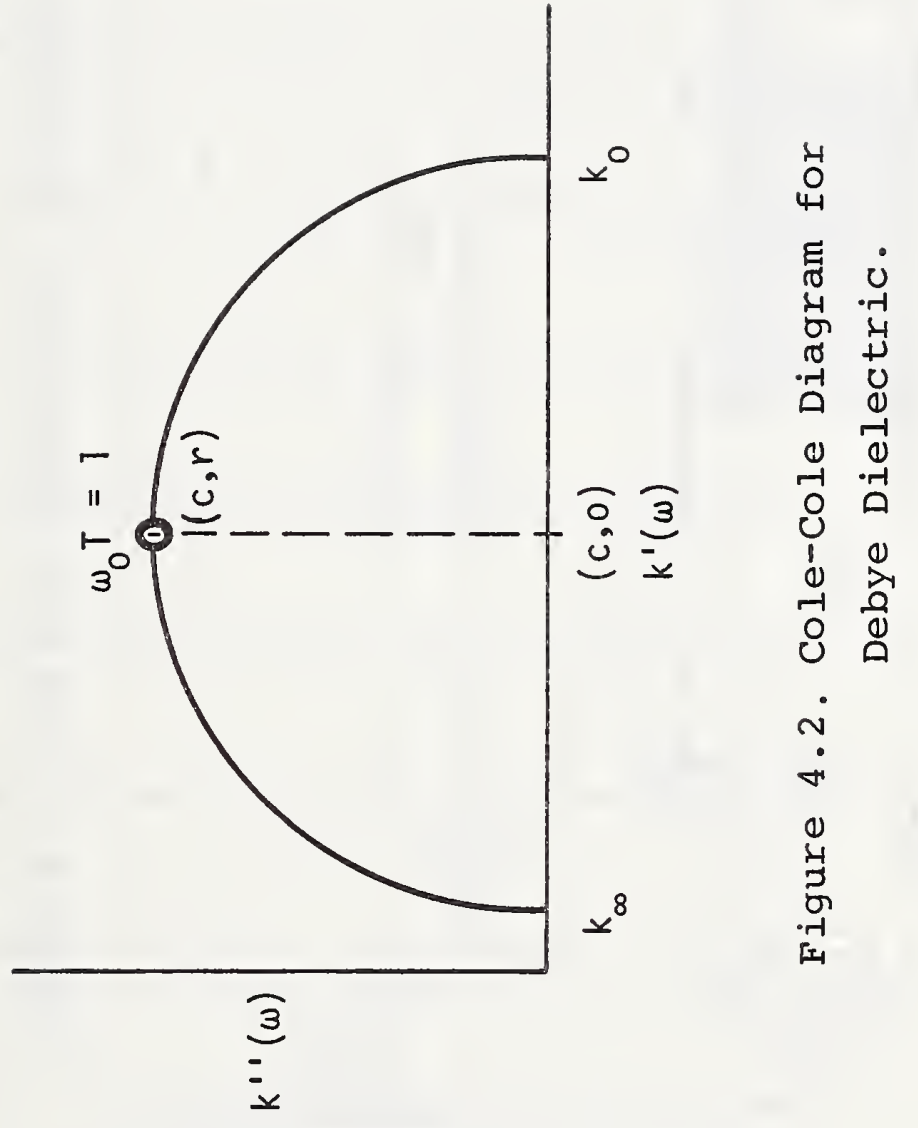



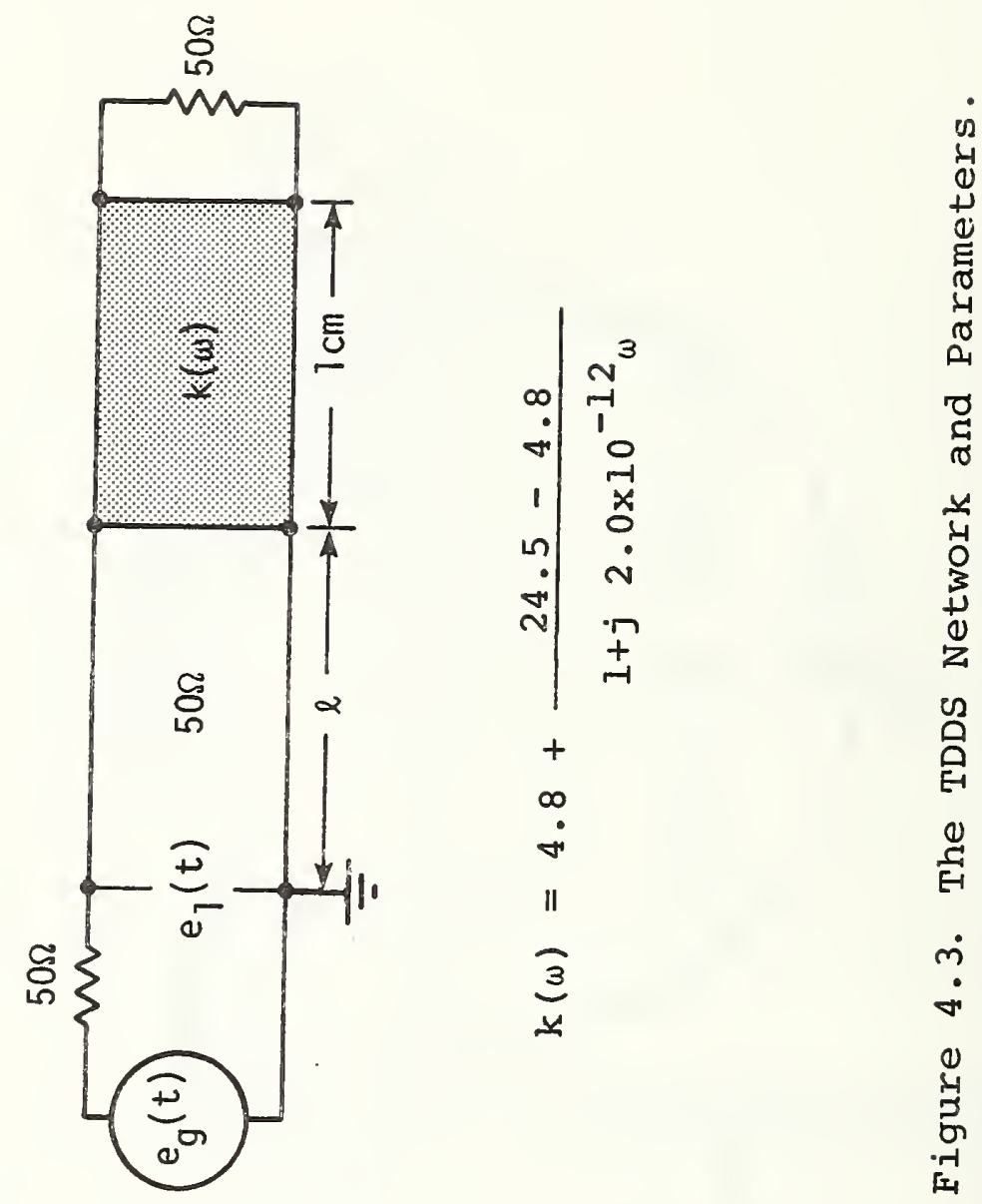


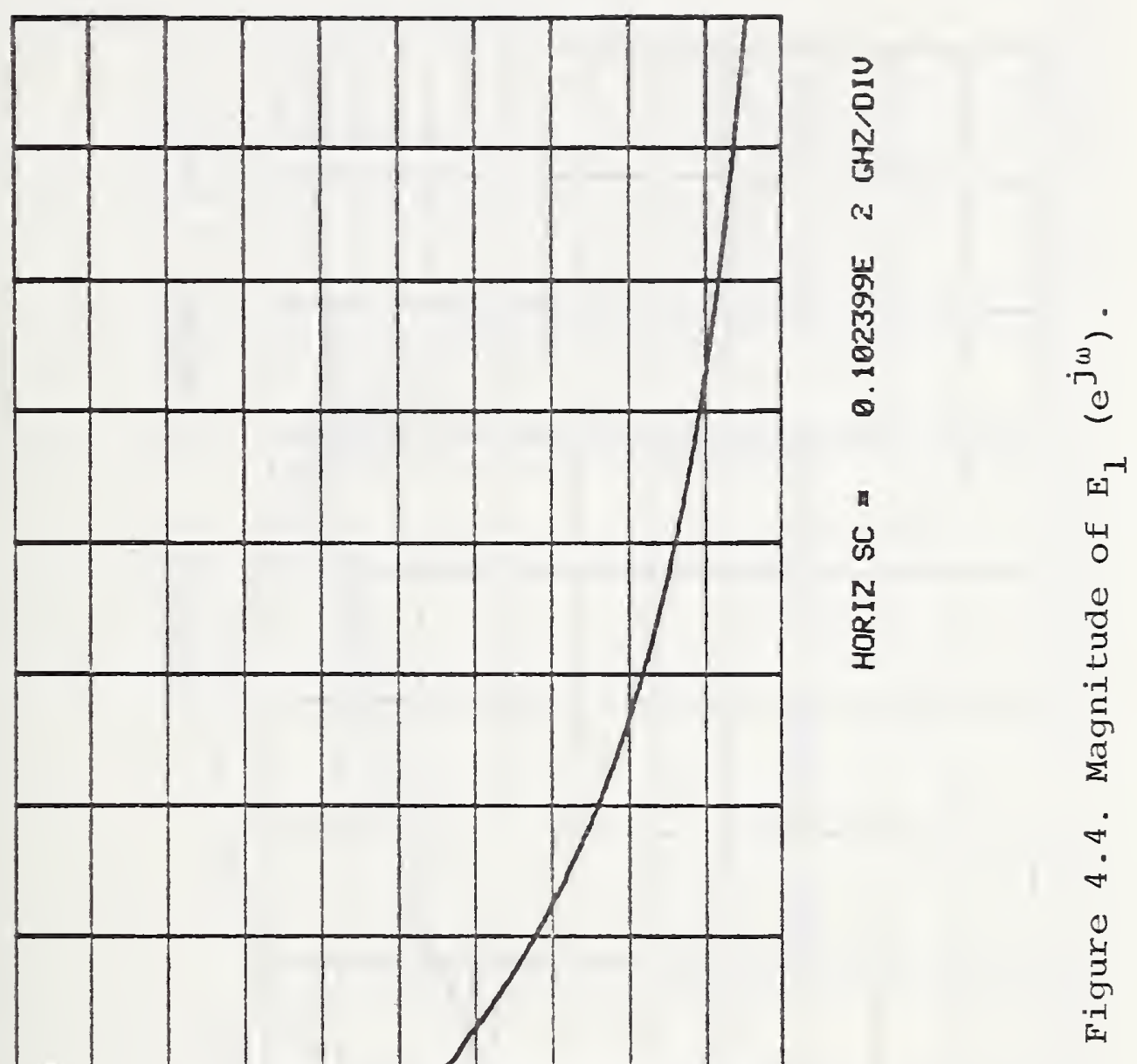


$\stackrel{3}{3}$
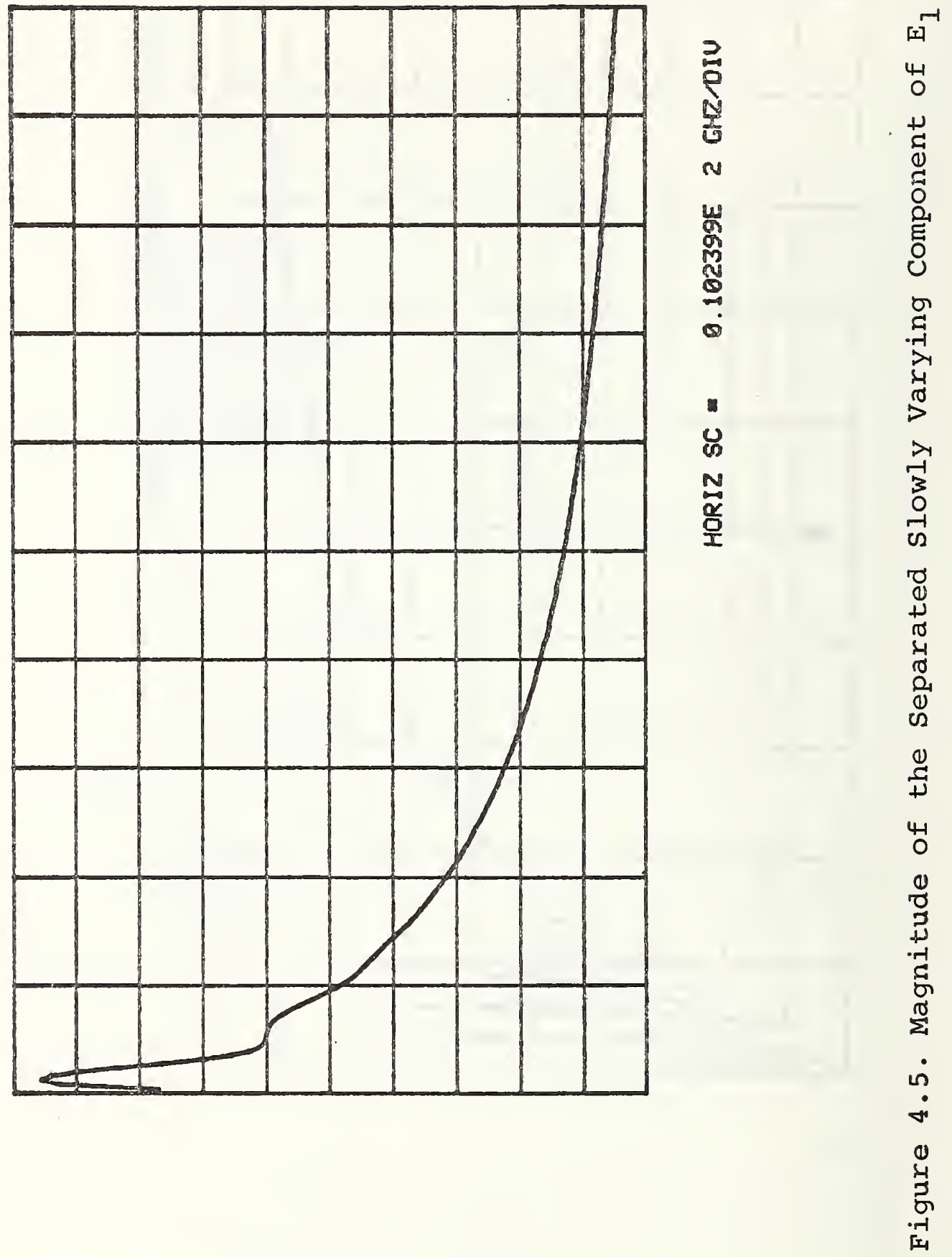


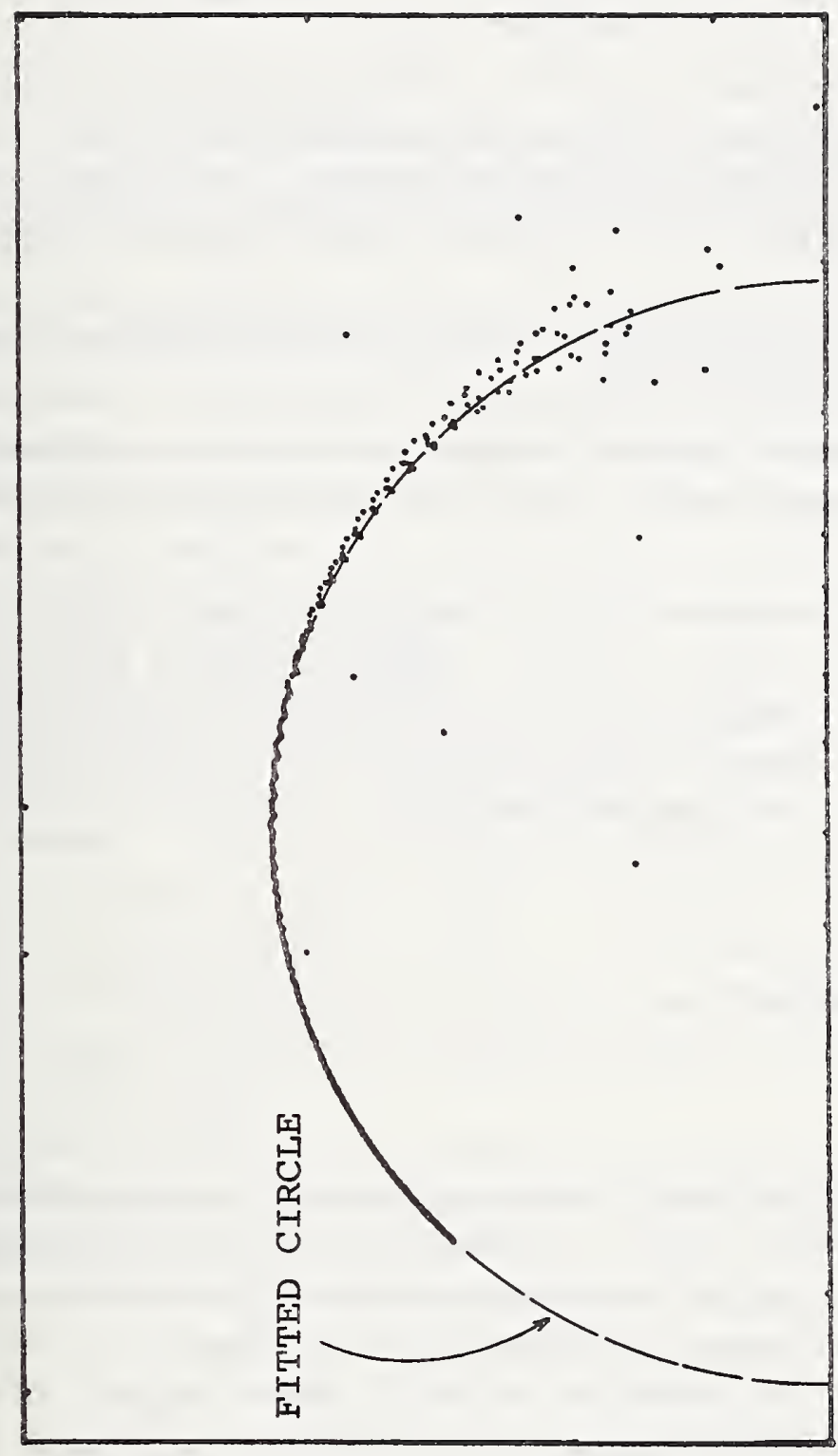

ल)

01

近

$\frac{1}{n}$

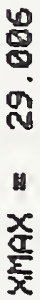

"ृ

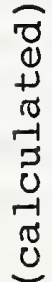

"

+
0
-1
01
0
-1
0
0
0
0
0
0

$\stackrel{\nabla}{\infty}$

$\dot{0}$

$\sum$

•" 
5. THE APPLICATION OF THE HOMOMORPHIC DECONVOLUTION TRANSFORM TO THE REMOVAL OF SCATTERING COMPONENTS FROM ANTENNA TEST RANGE DATA

\subsection{Introduction}

Time domain analysis of electromagnetic radiation problems has become increasingly more popular in the recent years because the time domain method provides physical insight into the behaviour of antenna structures excited by pulsed sources. Time domain measurements do offer significant advantages over the classical steady-state frequency domain measurement; they are as follows:

a. There is no need for an anechoic chamber to absorb unwanted reflections because time-windowing can be shut-out reflections.

b. There is no need for repeated magnitude and phase measurements at different frequencies because the time domain waveform contains the complex spectral information in a single entity.

Frequency domain information can be obtained from the measured time domain waveform by simply computing the corresponding Fourler transform. However, a recently applied method [22, 23 and 24] based upon Prony's algorithm [25] can also be used to deduce the required frequency domain information. The method is based on the fact that the time-dependent function can be expressed as the sum of complex exponentials

$$
f(t)=\sum_{i} A_{i} e^{\alpha_{i} t}
$$

for which the frequency domain form is

$$
F(s)=\sum_{i} \frac{A_{i}}{s-\alpha_{i}}
$$

where $\alpha_{i}$ are the complex poles of $F(s)$ and $A_{i}$ are the corresponding complex amplitudes or residues. By fitting the time domain data to the form of eq. (5.1) and determining the location of the singularities $\alpha_{i}$ 's and the corresponding residues $A_{i}$ 's, the system under measurement is fully characterized according to classical circuit theory.

This method has the advantage of yielding an analytical spectral response for the experimental system rather than the discrete form that the numerically implemented Fourier transform yields. Also, it is capable of extrapolating the measured transient response for both early and late times.

It is clear that the above discussion favors time domain measurements for pulsed electromagnetic radiating systems. Also, it is clear that it should be possible to windowout any unwanted reflections and scattering components. However, for cases where these undesired reflections or scattering components do overlap on the useful part of the time domain signal, the homomorphic deconvolution should enable the separation and the rejection of the unwanted components. 
The present chapter will demonstrate the power of the homomorphic deconvolution transform in separating and removing unwanted reflections and scattering components in a pulsed electromagnetic radiating system. The transform will be applied to radiation from a wideband horn antenna pulsed by baseband signal having frequency components out to beyond $6 \mathrm{GHz}$.

\subsection{The Homomorphic Deconvolution as Applied to Antenna System}

The homomorphic deconvolution transform was developed earlier in chapter 2, and was used in chapters 3 and 4 for the separation of multiple reflections due to different discontinuities on a transmission line. In these cases point or discrete discontinuities generated reflections which transformed to clusters (discrete well-separated pulses) in the cepstrum-domain. The localized clusters were then separated by filtering.

In radiation systems, the situation is different. In addition to the point reflections there exist plane reflections, which can be called a continuium of multipaths. Also, there exist scattering components due to the interfaces between the antenna aperture and the radiation medium, and also those due to different nearby scattering objects. Figure 5.1 illustrates some of these phenomena.

Consequently, it is not expected that definite or point located clusters will appear in the cepstrum-domain of such systems; rather, it is expected that a distribution of contributions will appear in the cepstrum-domain. Figure 5.2 shows a typical cepstrum for antenna systems.* This, of course, makes the separation of unwanted components more difficult than the cases of chapters 3 and 4 . Accordingly, the degree of filtering must be determined by a trial and error procedure. If too much of the cepstrum spread components is removed, it may include parts of the useful signal information. On the other hand, not removing enough of the unwanted components may result in a remainder which overlaps the useful time domain signal.

The separation in the cesptrum domain then should be done on a successive trial basis using different filters; the results should be examined in the light of the physical system to decide upon the best filtering process.

The next section of the present chapter will be devoted to the description of the antenna system to be used in the forthcoming application.

\subsection{The Antenna System}

The experimental setup that was used is shown in figure 5.3. It consists of two wideband antenna horns set as a transmitting receiving pair, an impulse generator feeding the transmitting horn, and a wideband sampling oscilloscope connected at the output port of the receiving antenna to display the received waveforms. A layer of absorbing material was placed such that it would prevent reflections from the floor. When reflections were desired, the absorbing material was replaced by a large metallic sheet.

*Figure 5.2 may be compared with figure 3.10 to show cepstrum spread as compared to clusters. 
The antenna horns [26] are Identical in their physical structure which is shown in figure 5.4. The horn is designed in a symmetrical fashion around a center ground plane. The antenna presents a 50 ohm driving point impedance which minimizes reflections in the 50 ohm transmission lines associated with the transmitter (impulse generator) and receiver (oscilloscope).

The impulse generator has a $50 \mathrm{ohm}$ source impedance and generates the pulse shown in figure 5.5. The oscilloscope sampling head terminates the receiving transmission line in $50 \mathrm{ohm}$.

\subsection{The Application}

The output signal of the receiving horn of the setup shown in figure 5.3, with the absorbing material in place, was acquired and is shown in figure 5.6. The figure shows some reflection and scattering components not overlapping the main signal. Therefore, ordinary time windowing can be used to remove these unwanted components. However, the homomorphic deconvolution transform will be applied for the purpose of demonstrating the effect of the separating filter on the separated output signal.

Applying the homomorphic deconvolution transform on this signal, the cepstrum of figure 5.7 is obtained, which, as was discussed earlier, contains a spread of contributions rather than discrete clusters. The separation process was tried twice on this cepstrum using different filters having the half-widths $A$ and $B$, respectively, as indicated in figure 5.7 (the filters were symmetric about the cepstrum origin). The result of the first separation at position $A$ is given in figure 5.8, which may be compared with figure 5.9 where the reflections were time-windowed out at the indicated point on the figure. The two results very closely match, while the separation at $B$, shown in figure 5.10 , matches the time-windowed signal of figure 5.11. From these figures it can be concluded that the narrower the window in the cepstrum the narrower the window in the corresponding time domain. Also, a too narrow window in the cepstrum will start distorting the main signal as is clear from separation $B$ where some distortion showed up just preceding the main signal, figure $5.10 \mathrm{~b}$.

After experimenting with the above separation trials, it is interesting now to try a case where the reflections overlap the main signal. This was achieved by introducing a large metallic plate in place of the absorbing material in the setup of figure 5.3. The observed waveform is given in figure 5.12 where it is possible to see the reflections from the metallic plate overlapping the main signal. The corresponding cepstrum, obtained by the application of the homomorphic transform, is shown in figure 5.13. Two separations were used again at the windows indicated in the figure. The results of the two separations are given in figures 5.14 and 5.15. The two separation results are slightly different from each other and from the clean signal of either figure 5.9 or 5.11 , but it is important to notice that the introduced overlapping reflections (by the metallic plate) were successfully removed through the use of the homomorphic deconvolution transform. 


\subsection{Summary and Conclusions}

The present chapter began with an introduction about the role that the time domain measurement techniques can play in solving electromagnetic radiation problems. Then the distributed nature of the expected cepstrum in such problems was discussed and compared with previously studied discrete cases. Two examples of applying the homomorphic deconvolution transform to an antenna system were presented, and the transform proved to be very successful in the removal of the unwanted reflections and scatcering components either overlapping or not overlapping the main signal containing the desired signal information. 

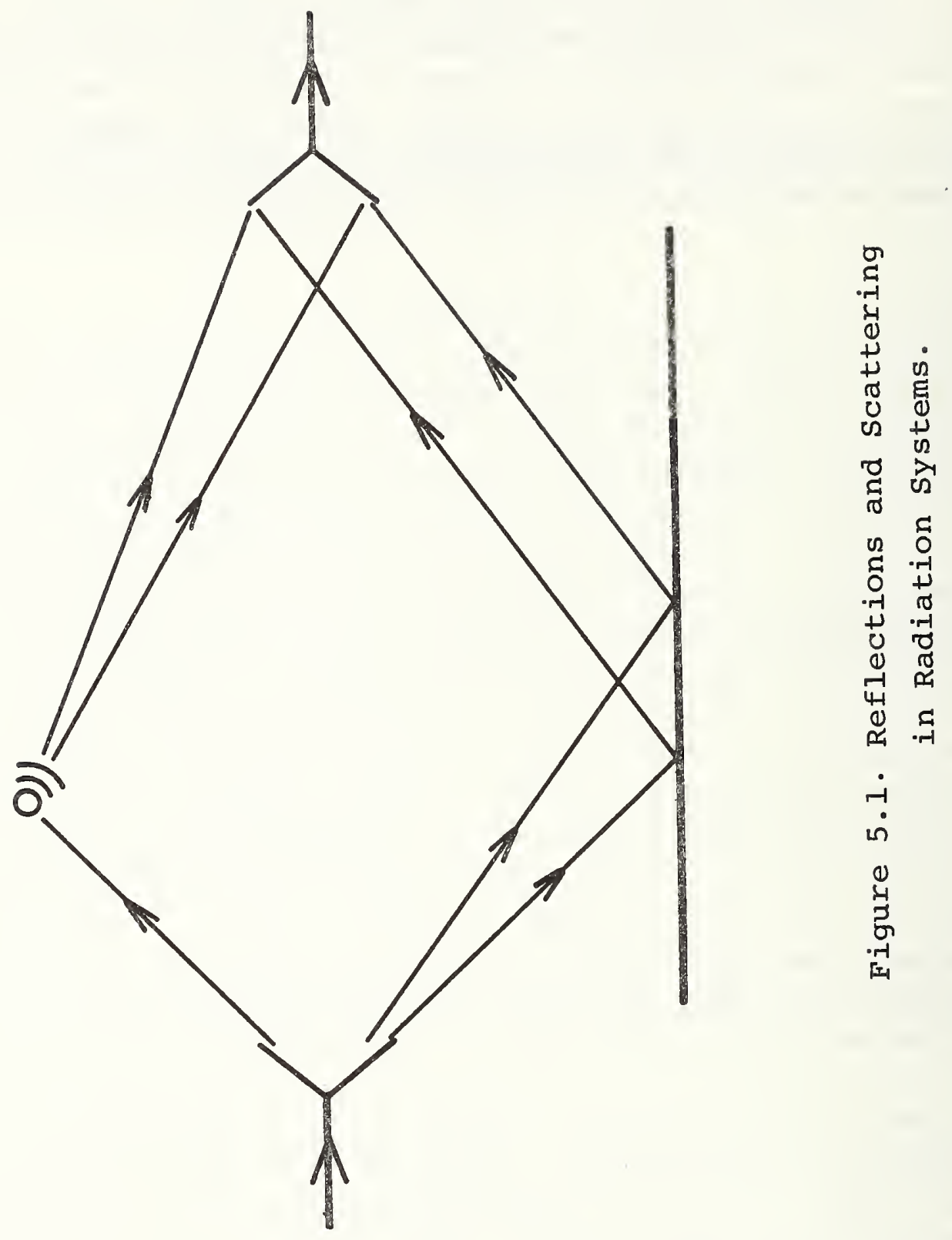

$-94-$ 


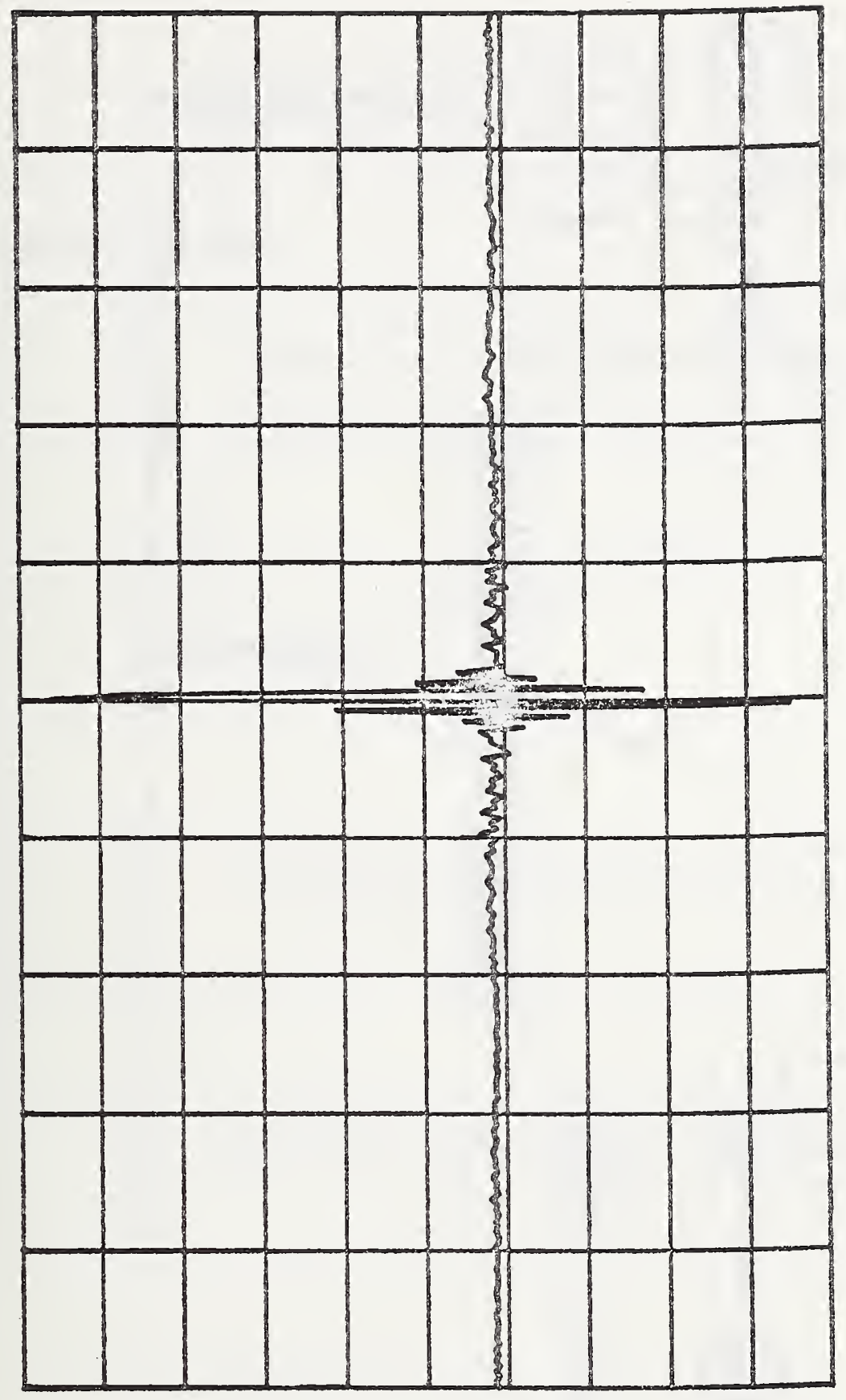

ป 


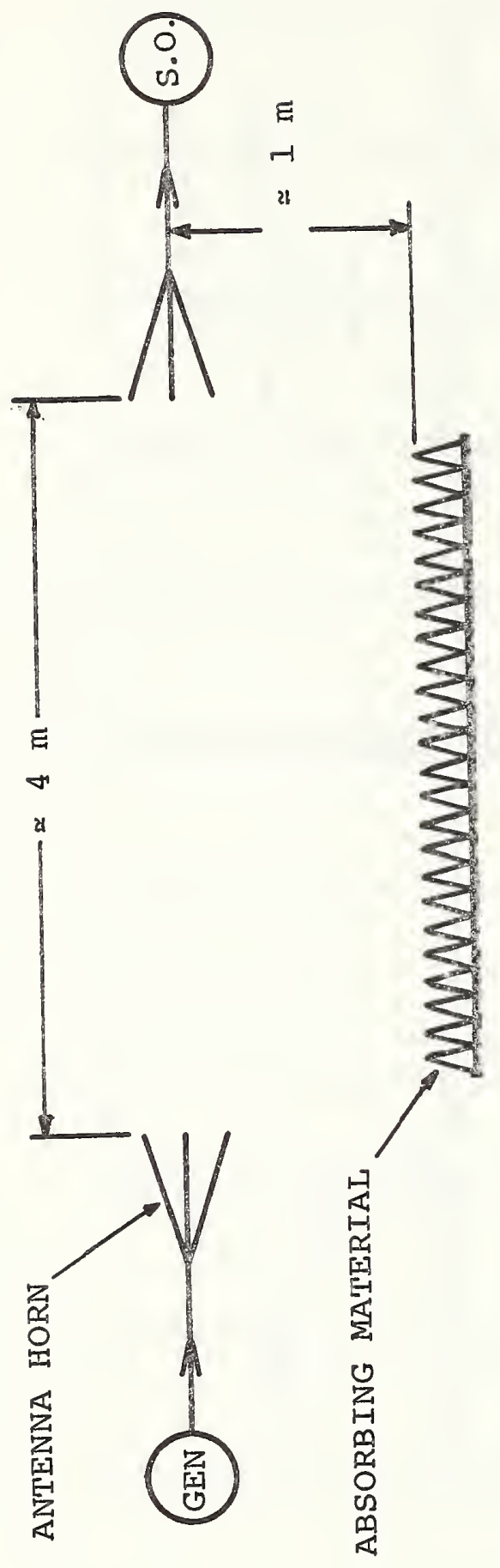

$\stackrel{0}{b}$

1
0

年

हु

is

बू

菖

音

m

岌 
DIELECTRIC FILLING

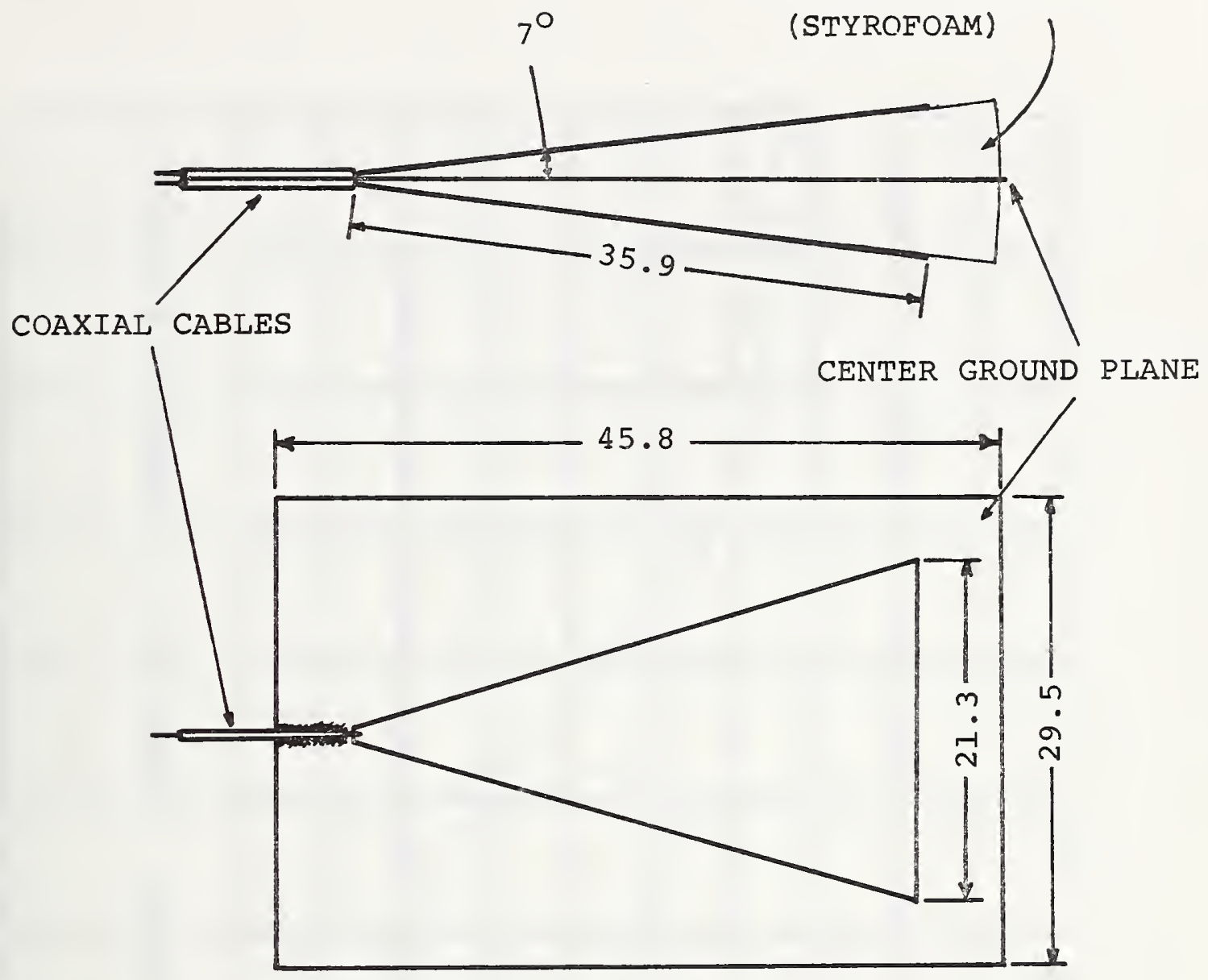

Figure 5.4. The Wideband Antenna Horn. (all dimension in $\mathrm{cm}$ ). 


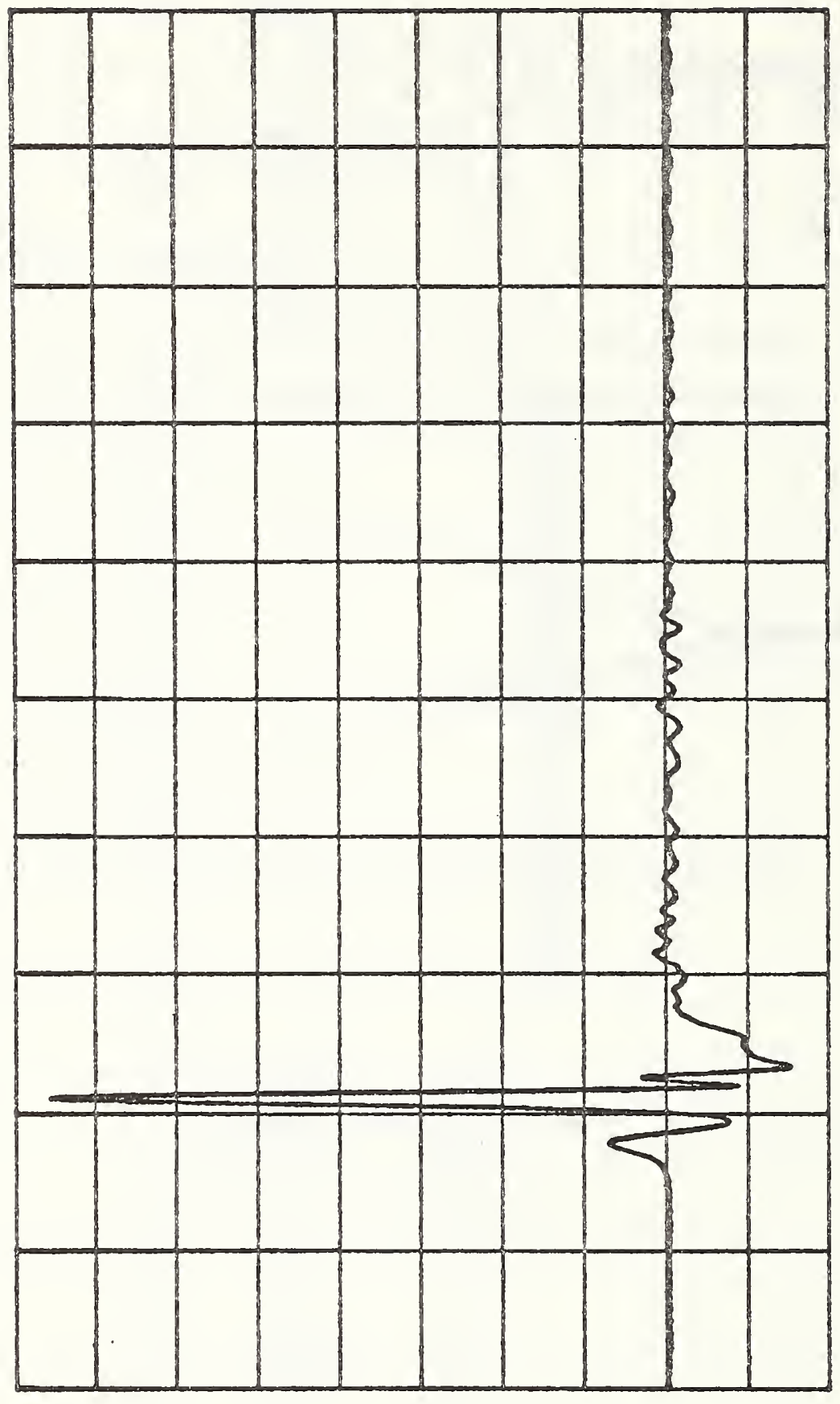

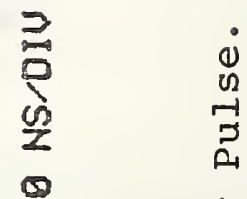

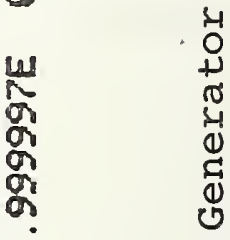

0

岁

$\frac{1}{N}$

$4-1$

ह્મ

$\stackrel{w}{\sim}$

in

.

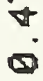

กั

เก

范

䓀 

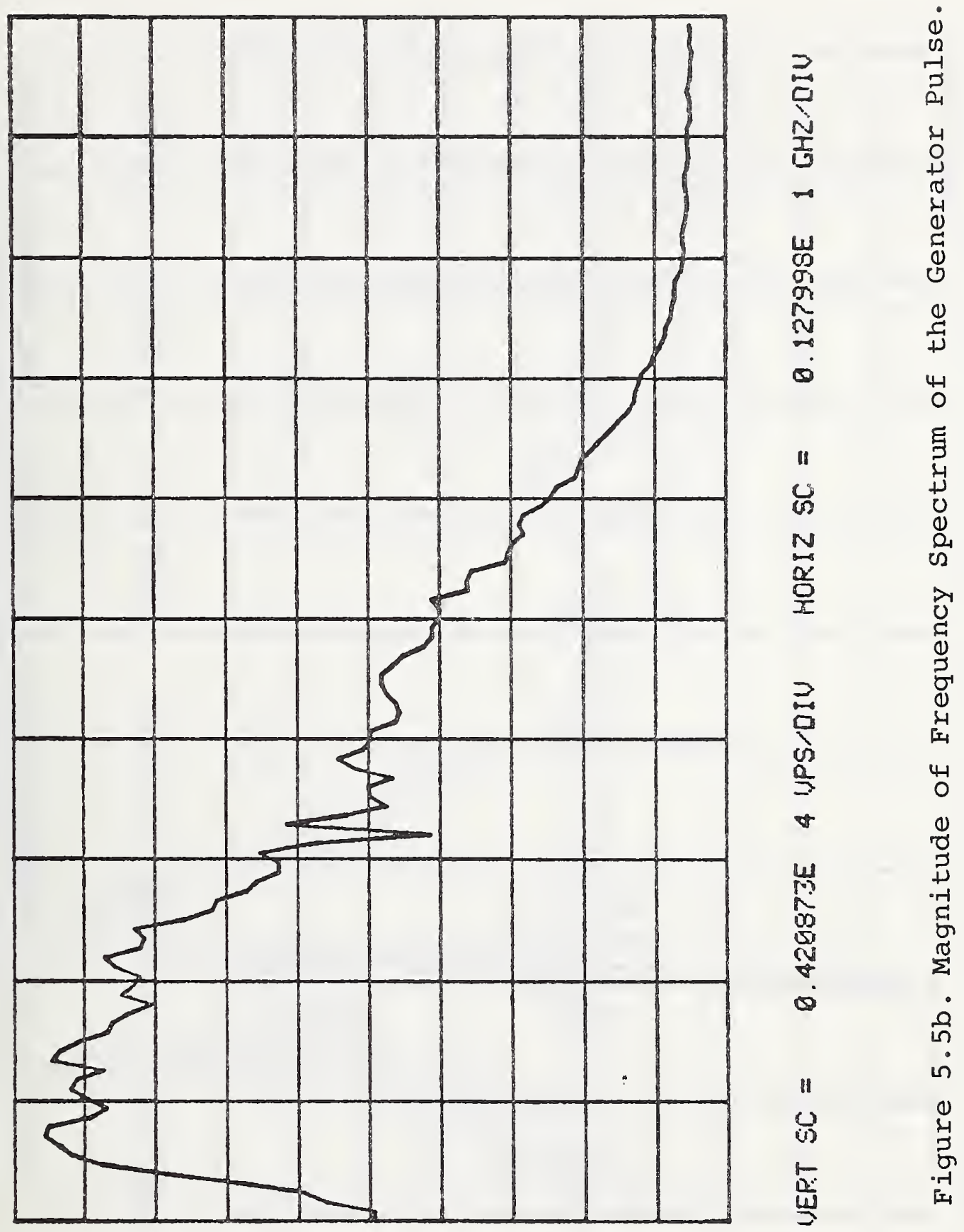


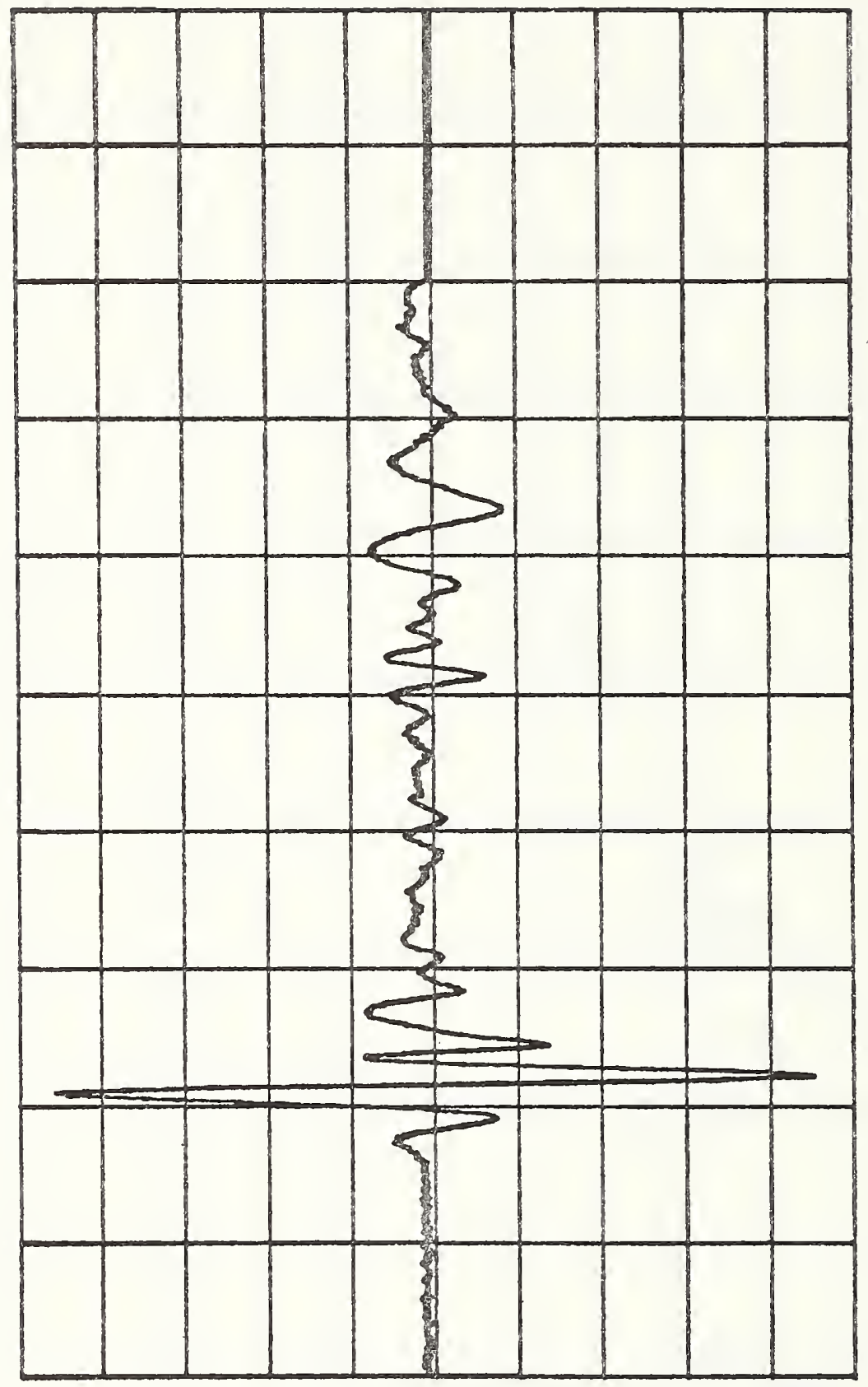

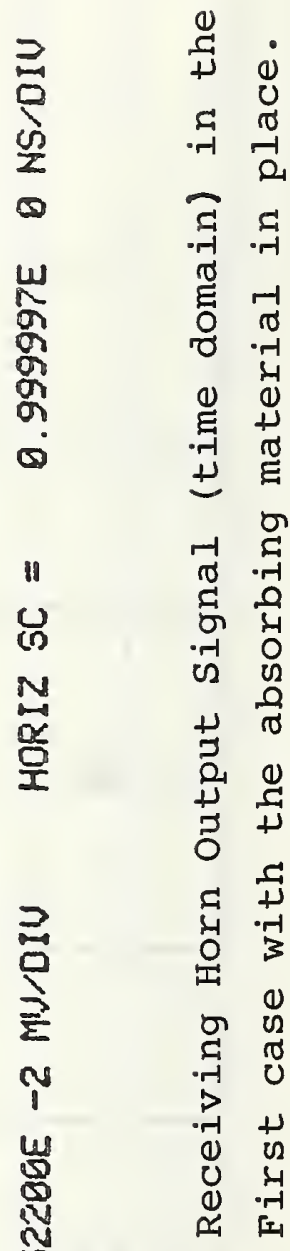

尔

ம்

11
0
$\frac{5}{3}$

豙 


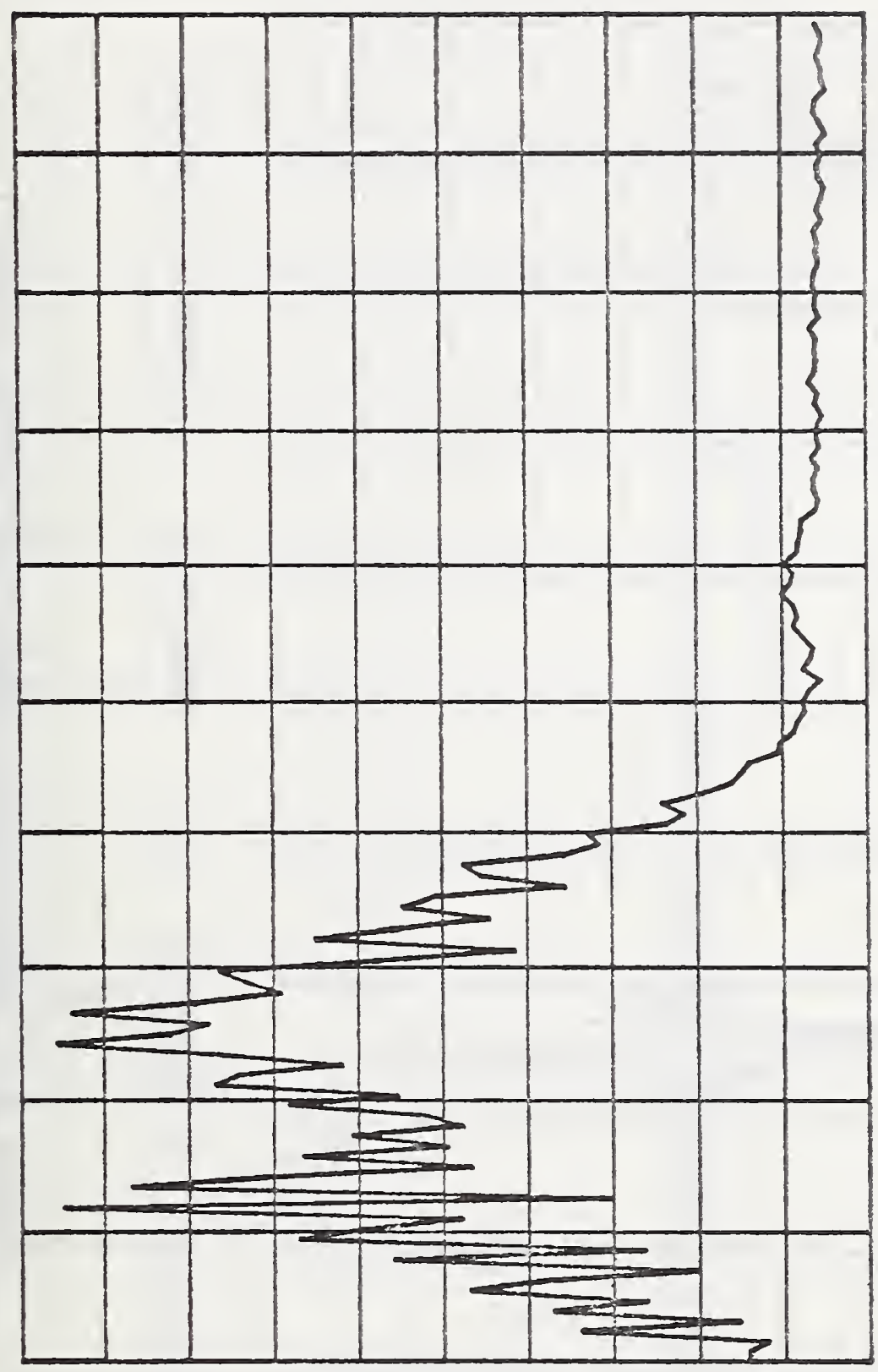

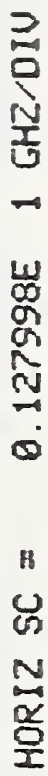

I

0

:

$\dot{0}$

2
0
0
0
0
0

त⿱

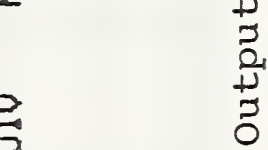

药

논

品

0
.1
-1
3
-1
0
0
0
0
01

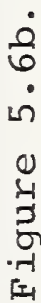




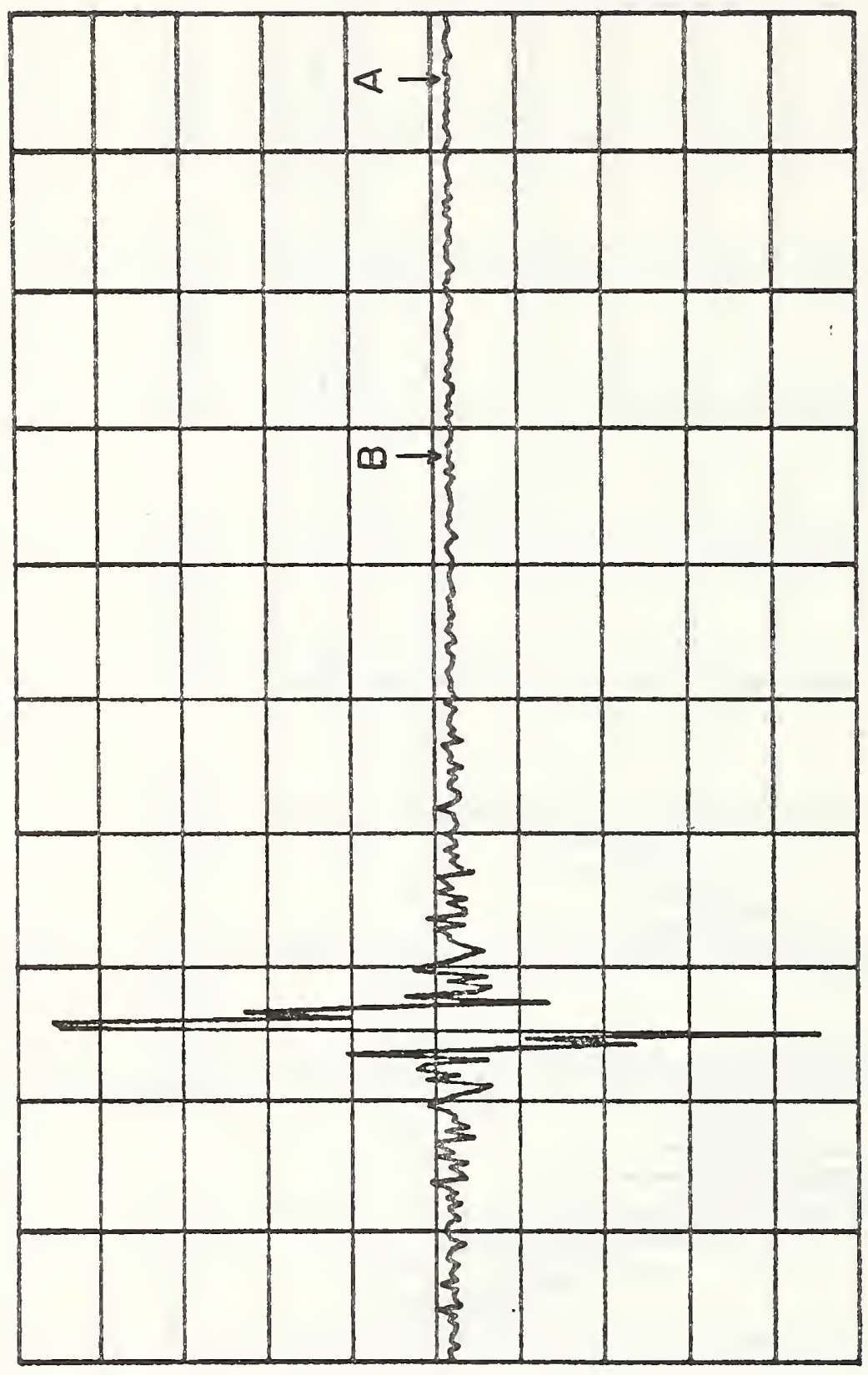

2
0
0
0
$w$
$\omega$
ज
\%
$\infty$
$\infty$

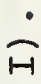

0
0
8
0

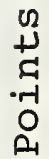

N

1
0
.1
0
0
0
0
0
0
0
0

ర్

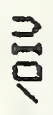

E⿱

虫

مि

क

눈 


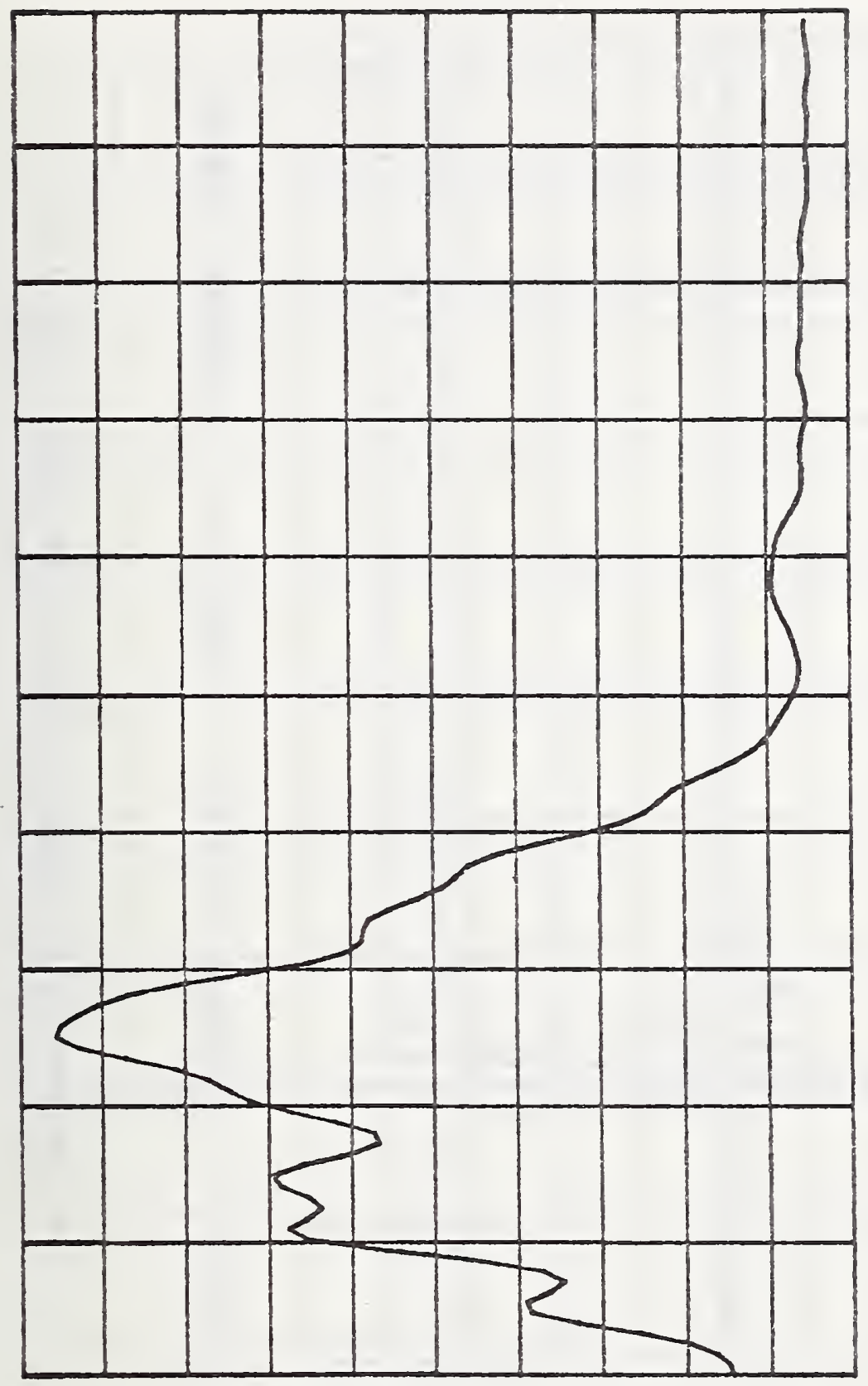

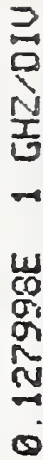

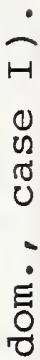

$n$
0
0
0
0
0
0

نั

它

$\begin{array}{ll}3 & 0 \\ 0 & 0 \\ 0 & +1 \\ 0 & 0 \\ 0 & 0 \\ 2 & 0 \\ 0 & 0 \\ 0 & 0\end{array}$

\begin{tabular}{l}
$w$ \\
5 \\
\multirow{5}{*}{} \\
6 \\
0 \\
0
\end{tabular}

II

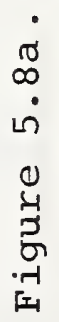

क)

珤 


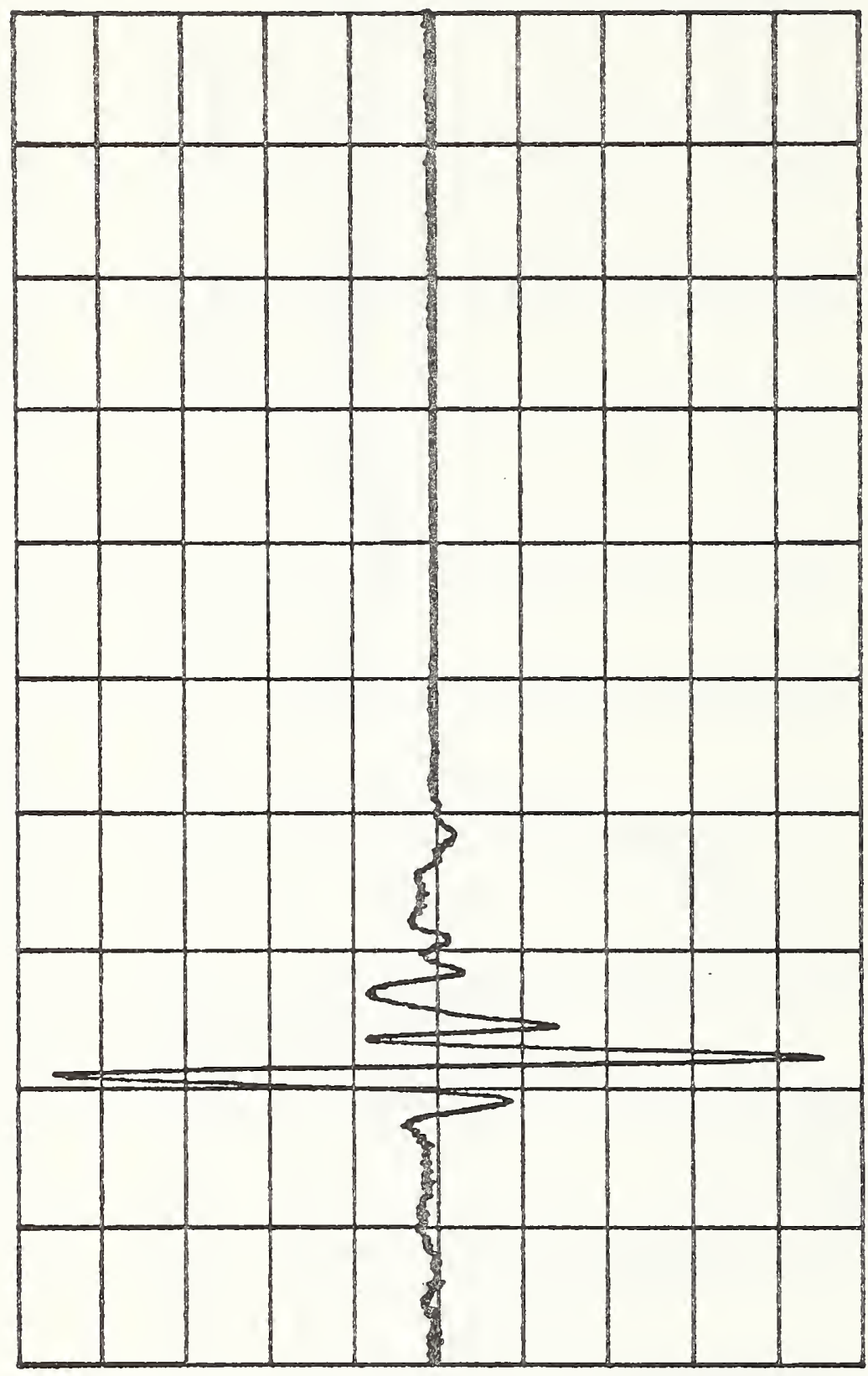

尔

F'

$\frac{0}{0}$

岂

4

동

$=$

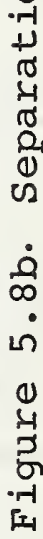

(1)

(5)

$\frac{0}{3}$ 


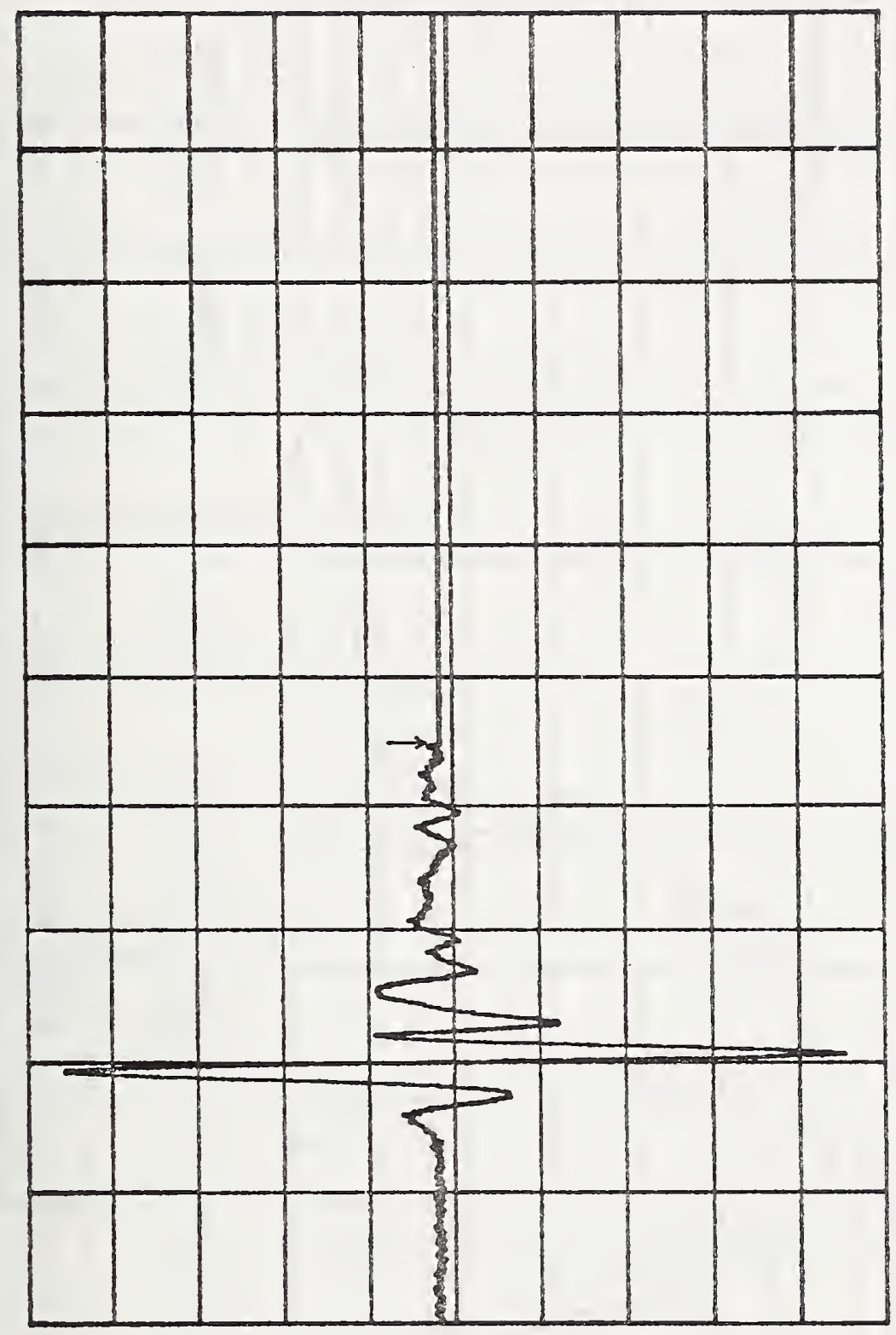

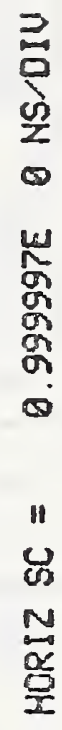

$\widehat{H}$

$\begin{array}{ll}2 & 0 \\ 0 & 0 \\ 0 & 0\end{array}$

-

غ્

r

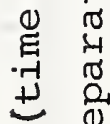

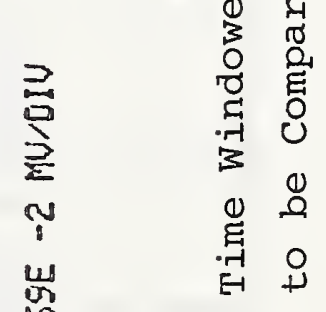

要

बं

告

- 13

तु

का मे

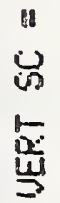

岌 


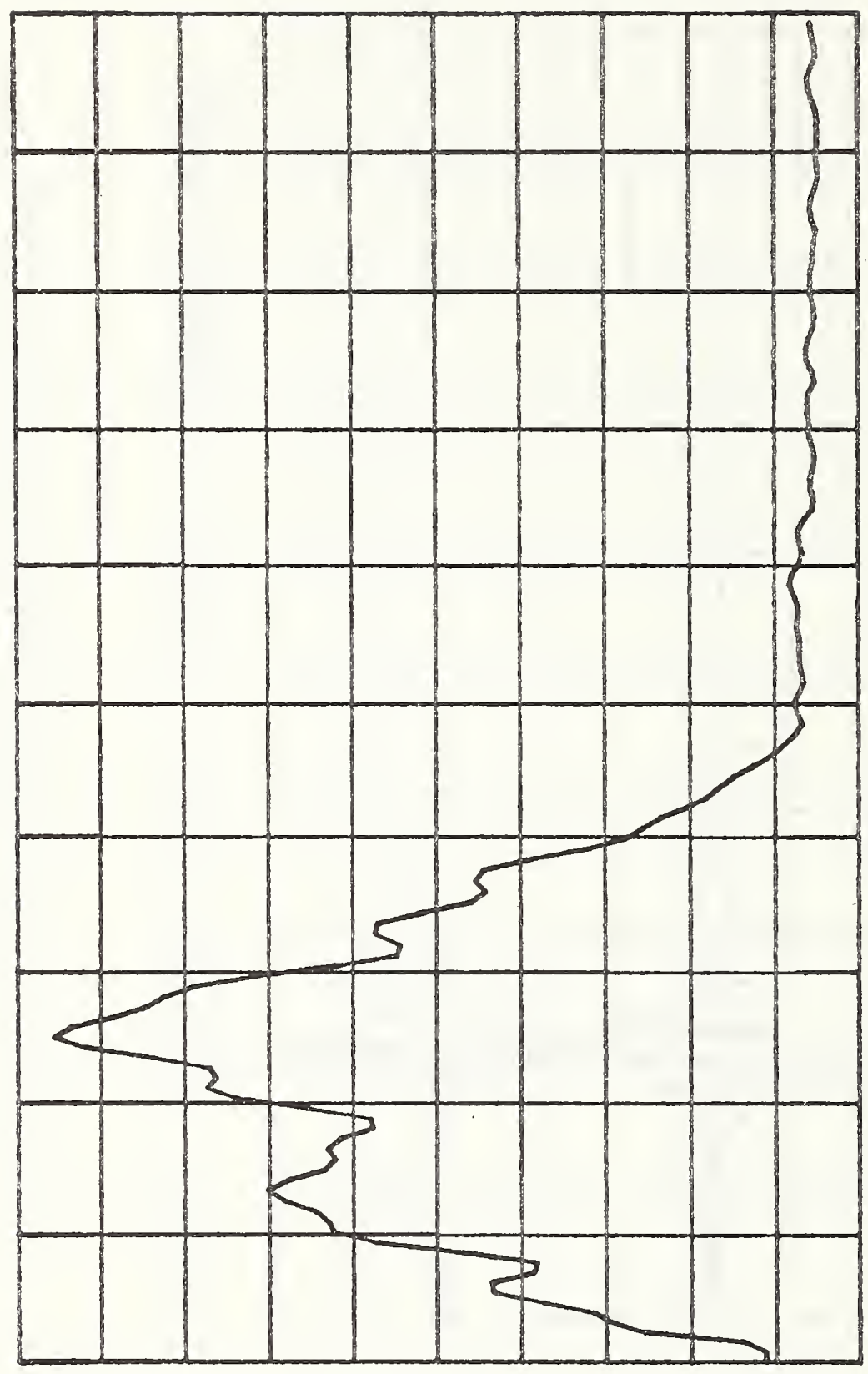

$\sum_{-\infty}^{2} \quad$ न1

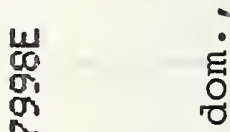

$\stackrel{N}{\infty}$

-1
0
0
0
0
0

ச்

先

4

ठே.

os

है ए

$\underset{2}{\alpha}$

苗

is 3

$\frac{0}{3}$

ठర

20

足

$\infty$

m

in

远

$\infty$

ตุ

"⿺辶冋

嵌 


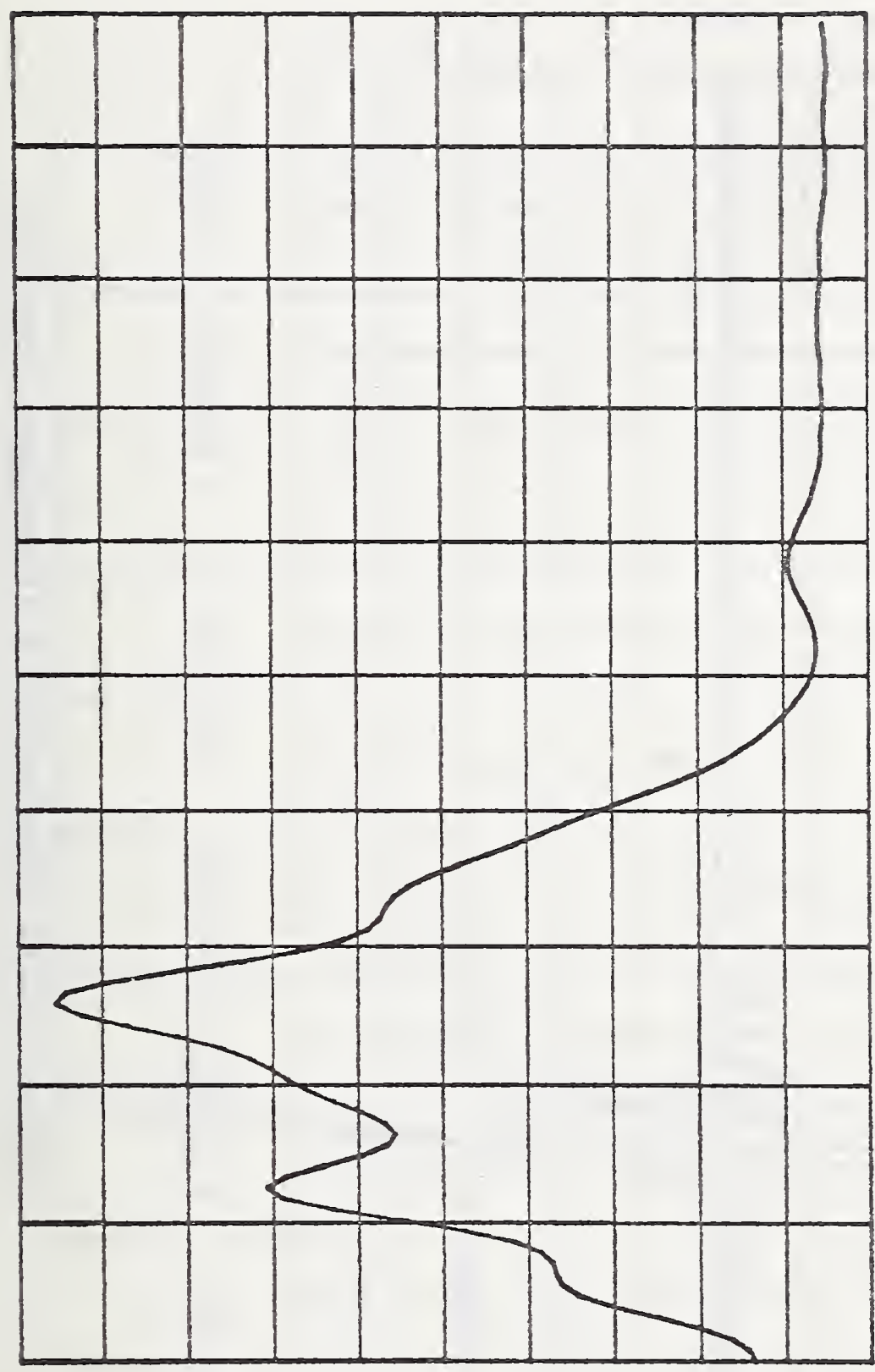

몬

0
1
0
0
0
0

N.

을

है

$\dot{8}$

官

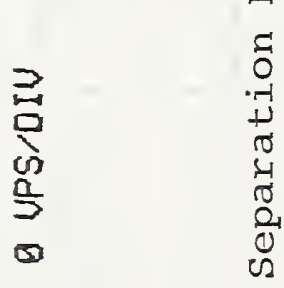

$\infty$

$\omega$
ñ
0
0
$\omega$
0

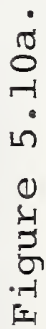

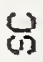

$\frac{5}{2}$ 


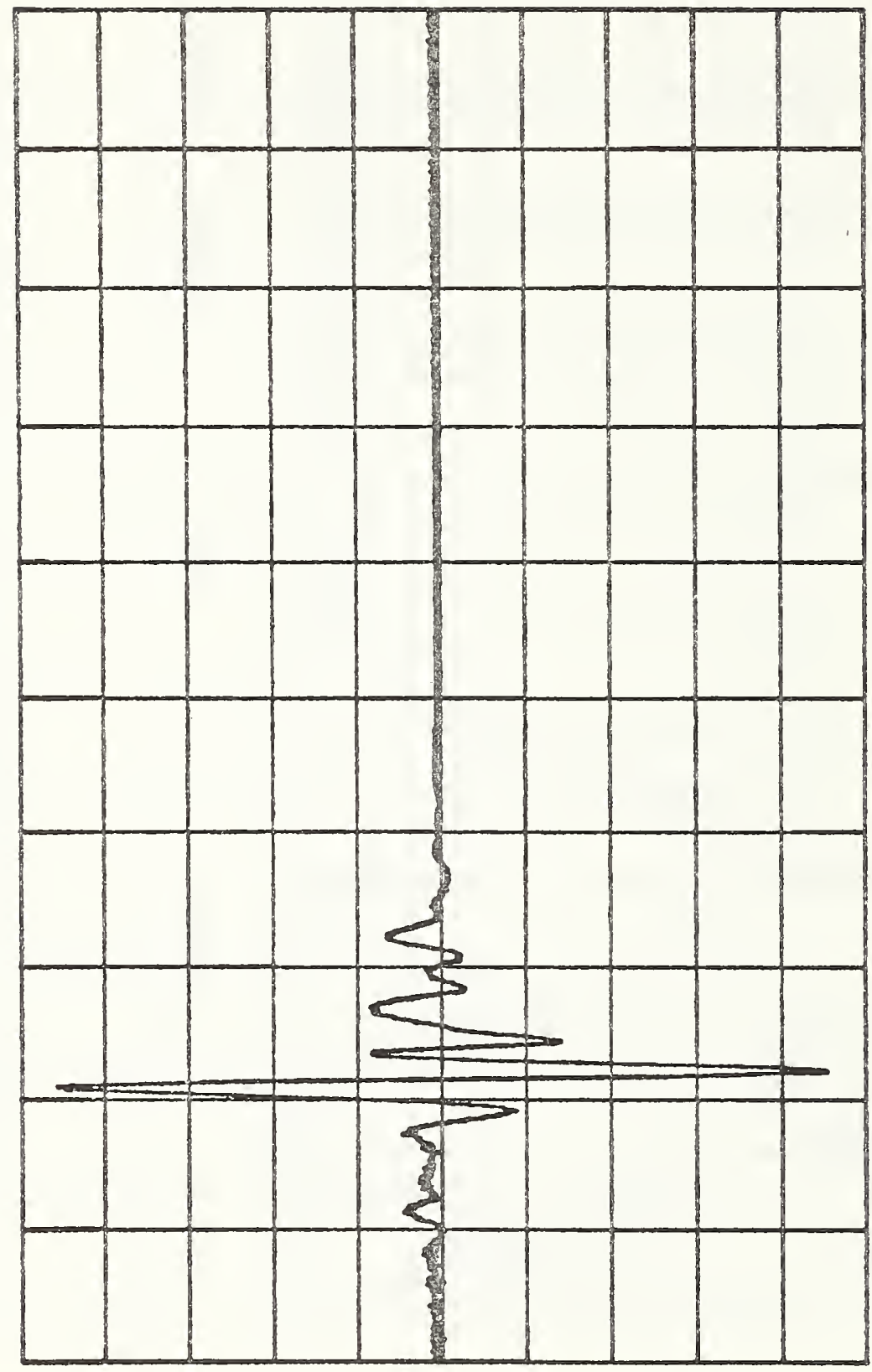

3
0
0
0
0
$w$
0
0
0
0
0

$\dot{1}$
0
0
0
0
$\vdots$
$\dot{E}$
0
0

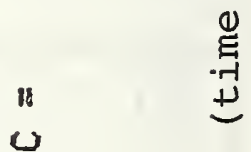

$\omega$

$\frac{N}{2}$

$\infty$

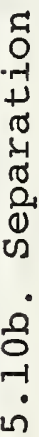

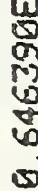

站

感 


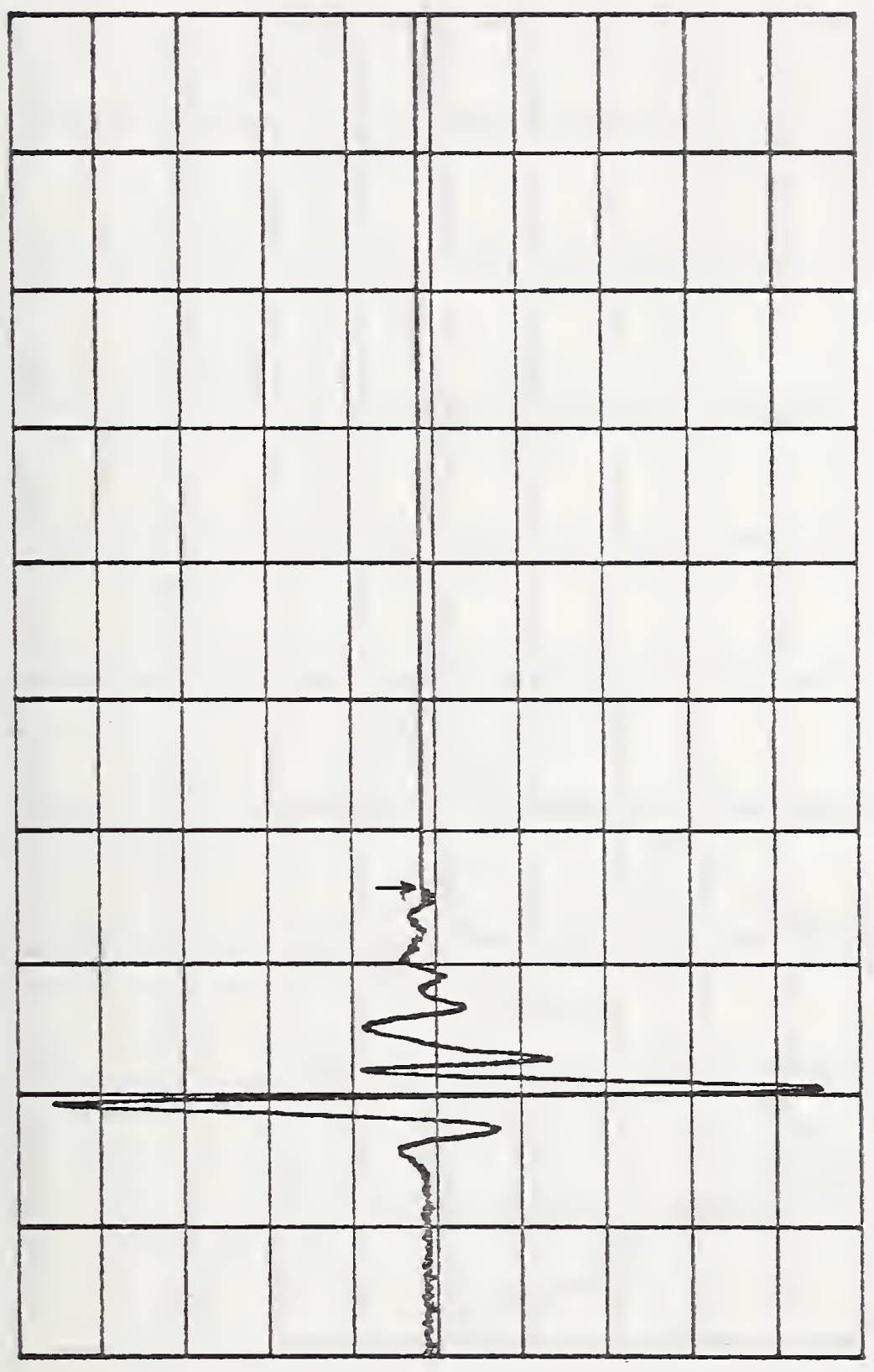

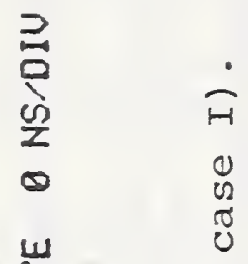

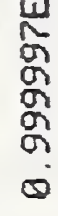

- $\dot{0}$

1 䓠

जs

웅

त्

$\begin{array}{cc}0 & 0 \\ 0 & 0 \\ 0 & 4 \\ 3 & 0 \\ 0 & 0 \\ 0 & 0 \\ 0 & 0 \\ -1 & 0 \\ 3 & 0 \\ 0 & 0 \\ 0 & 0 \\ -7 & 0 \\ -1 & 0\end{array}$

ก

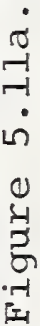

जे हु

皆 

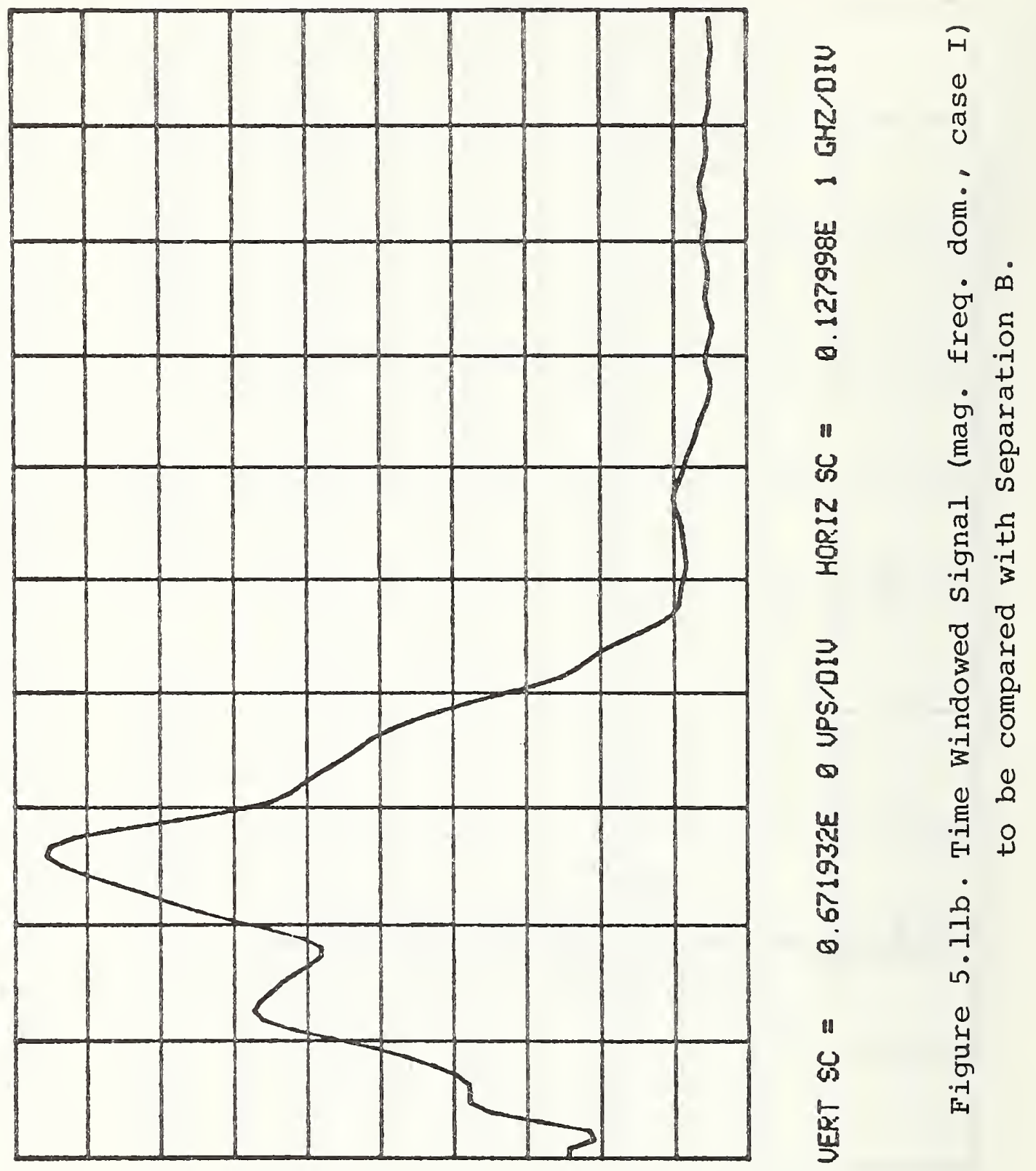


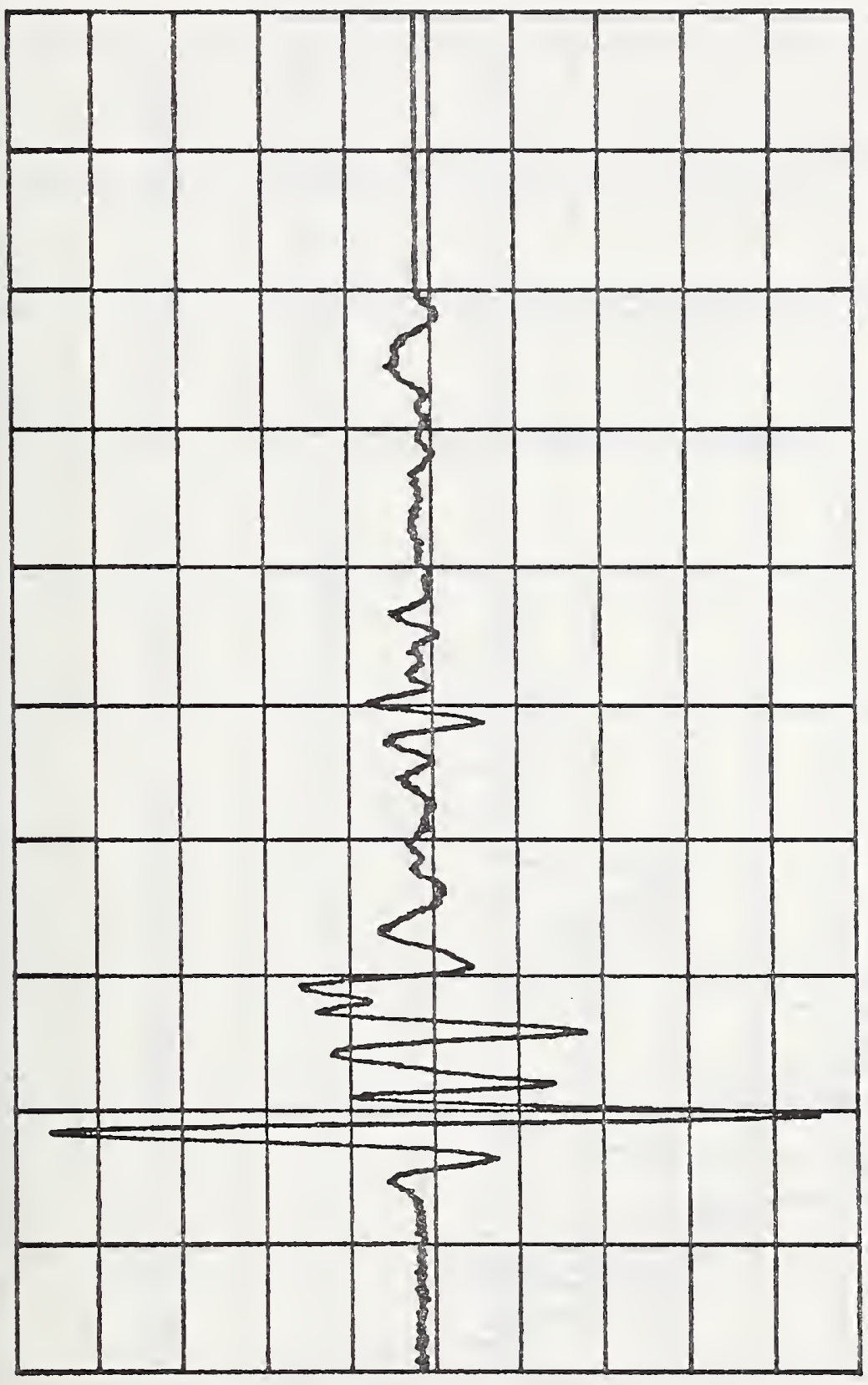

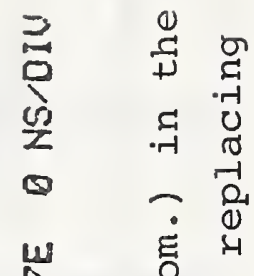

䋛 हु

on

व

के

(1) ठ ठ तु

क ज है

$N \quad+0$ न

잉 苟

-

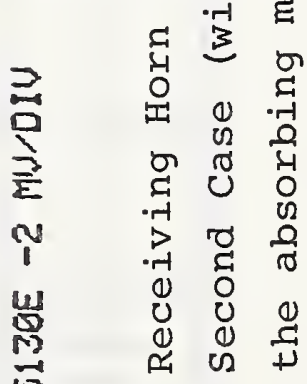

47.

๑) ণั

$\infty$.

เก

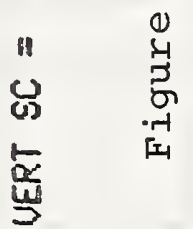



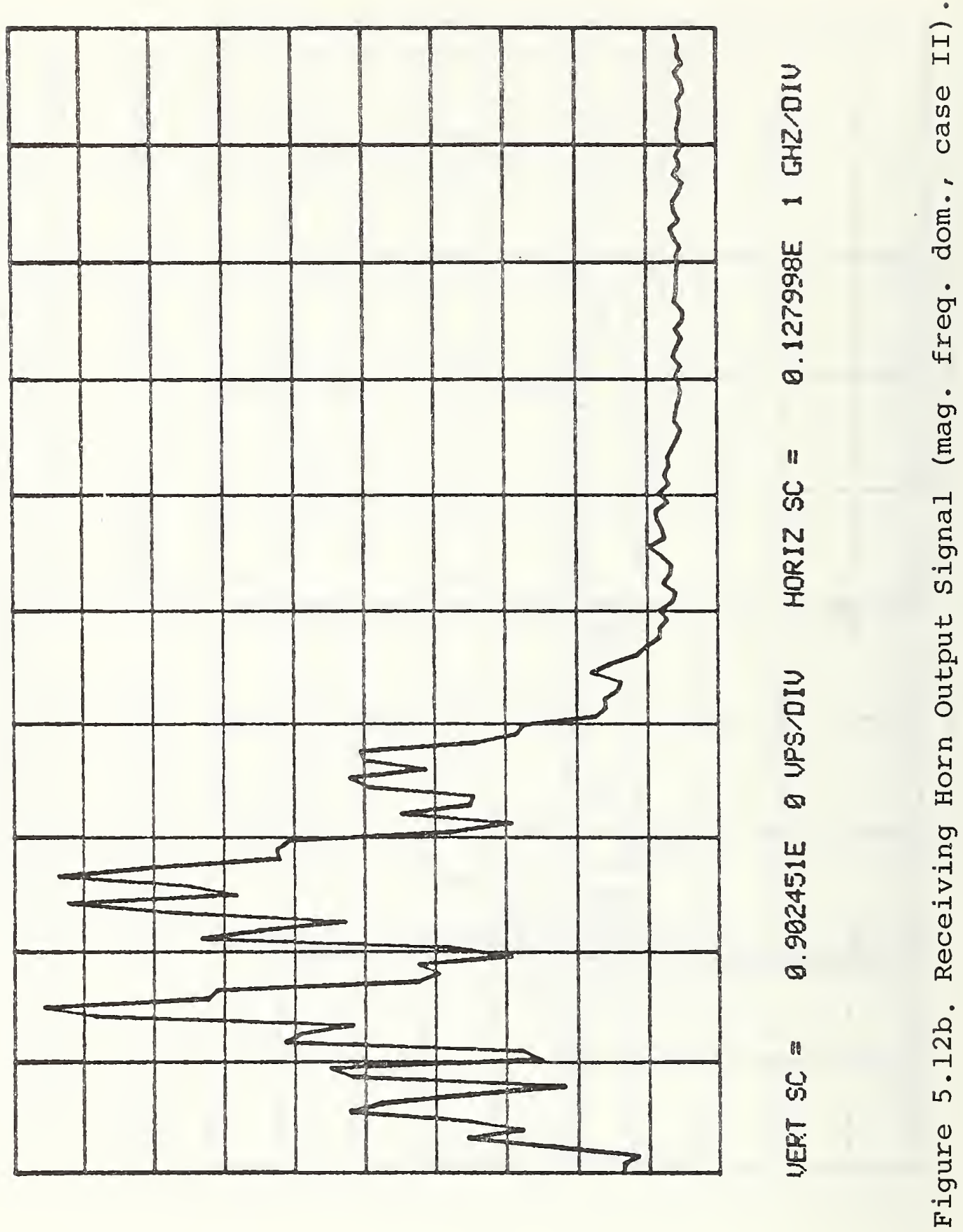


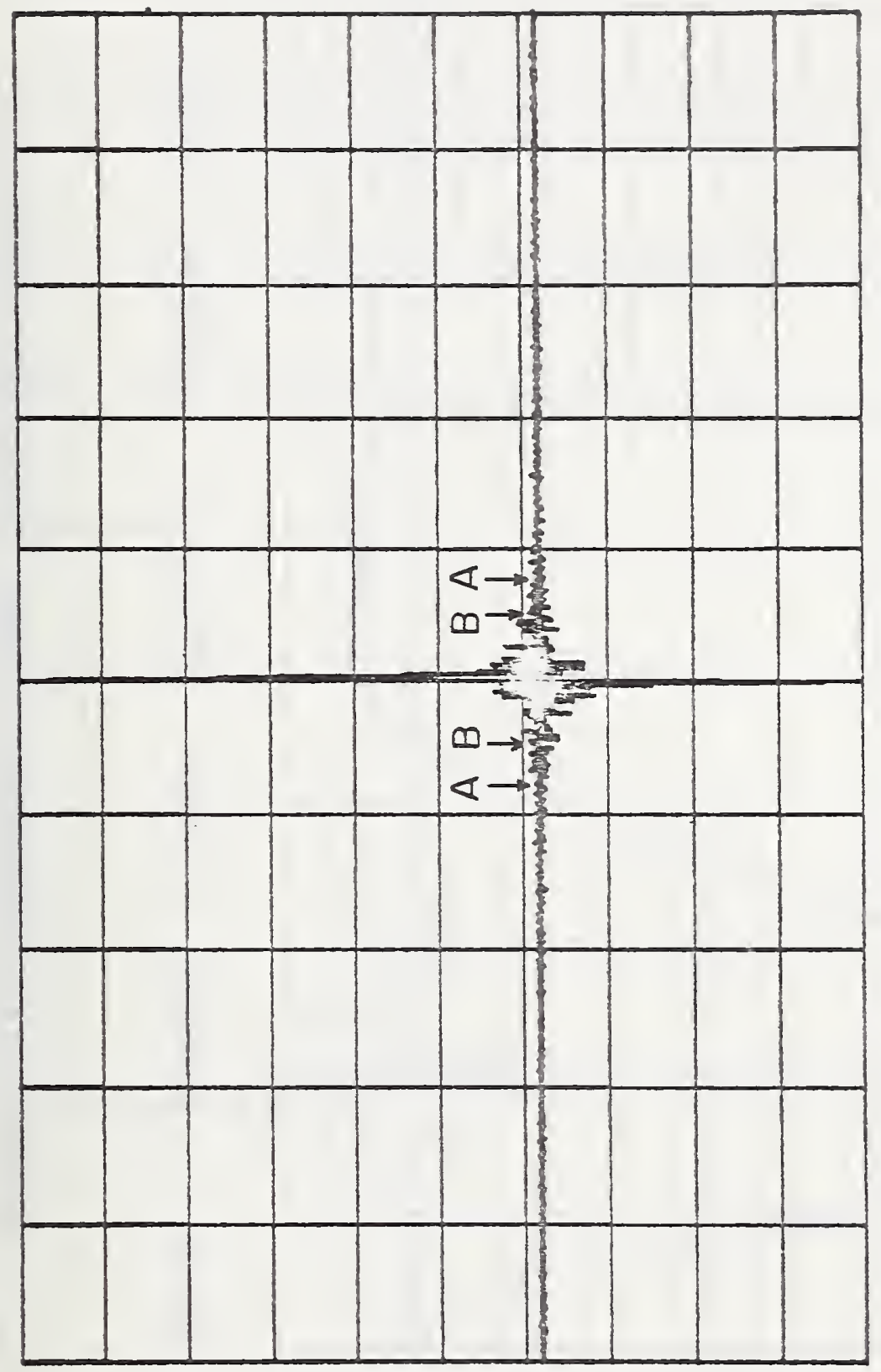

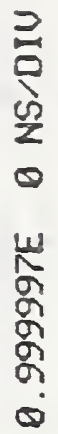

$\stackrel{\circ}{H}$

0
0
0
0

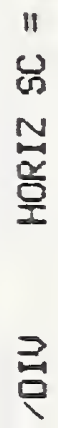

1

ธี

롤

1

ํㅗㄴ

II

(1)

悹 


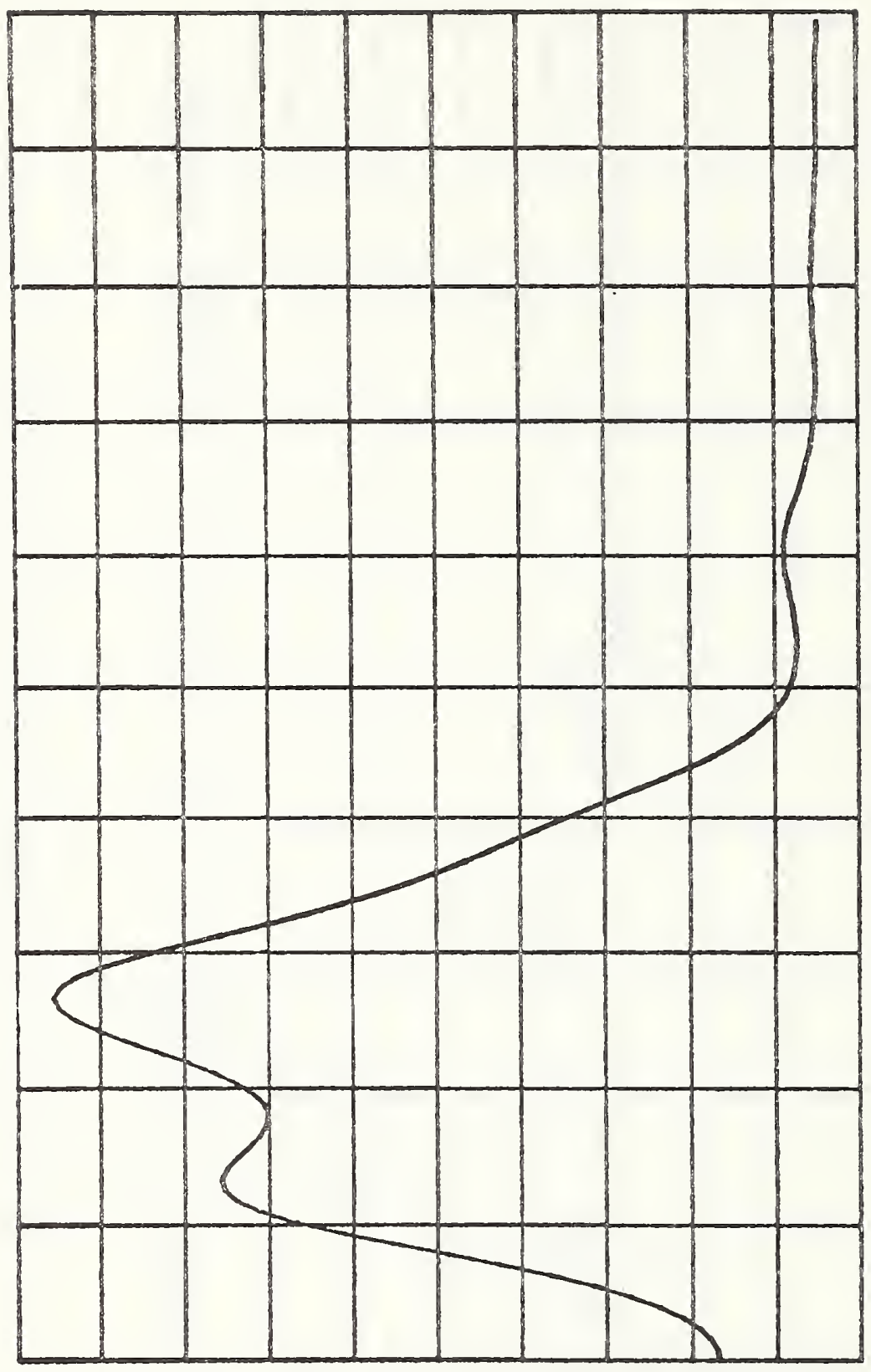

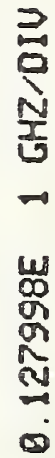

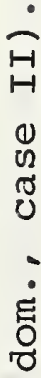

क
$\frac{0}{0}$
$\frac{\alpha}{x}$

0
0
4
4

它

0
0
0
0

ב્1

0
0
0
0
0

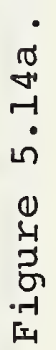

co

$\frac{5}{2}$ 


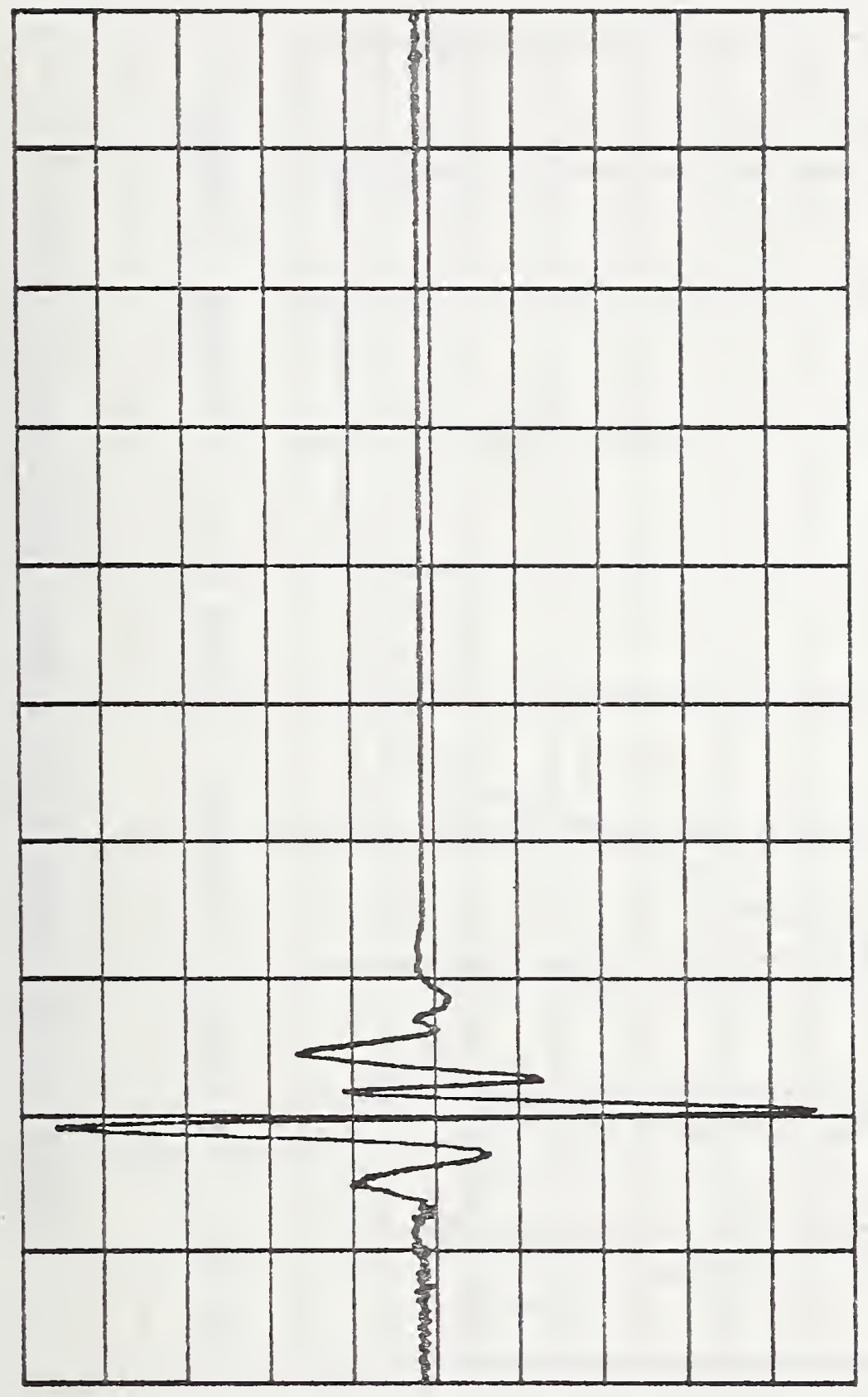

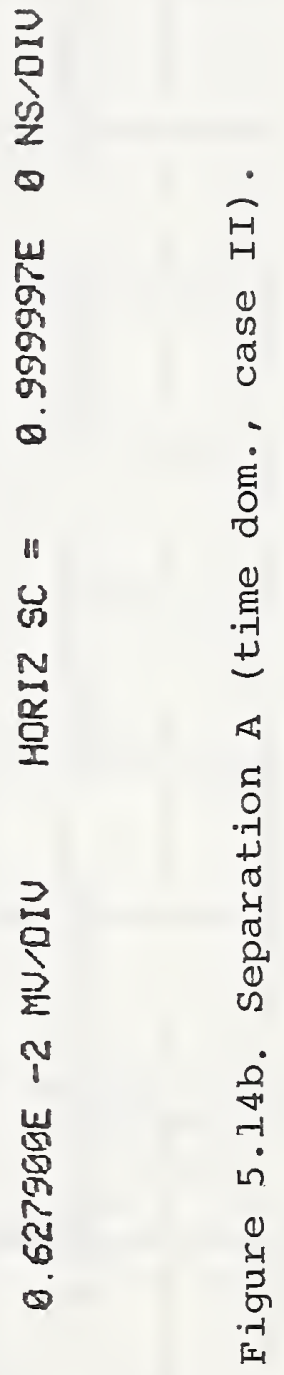

峁 

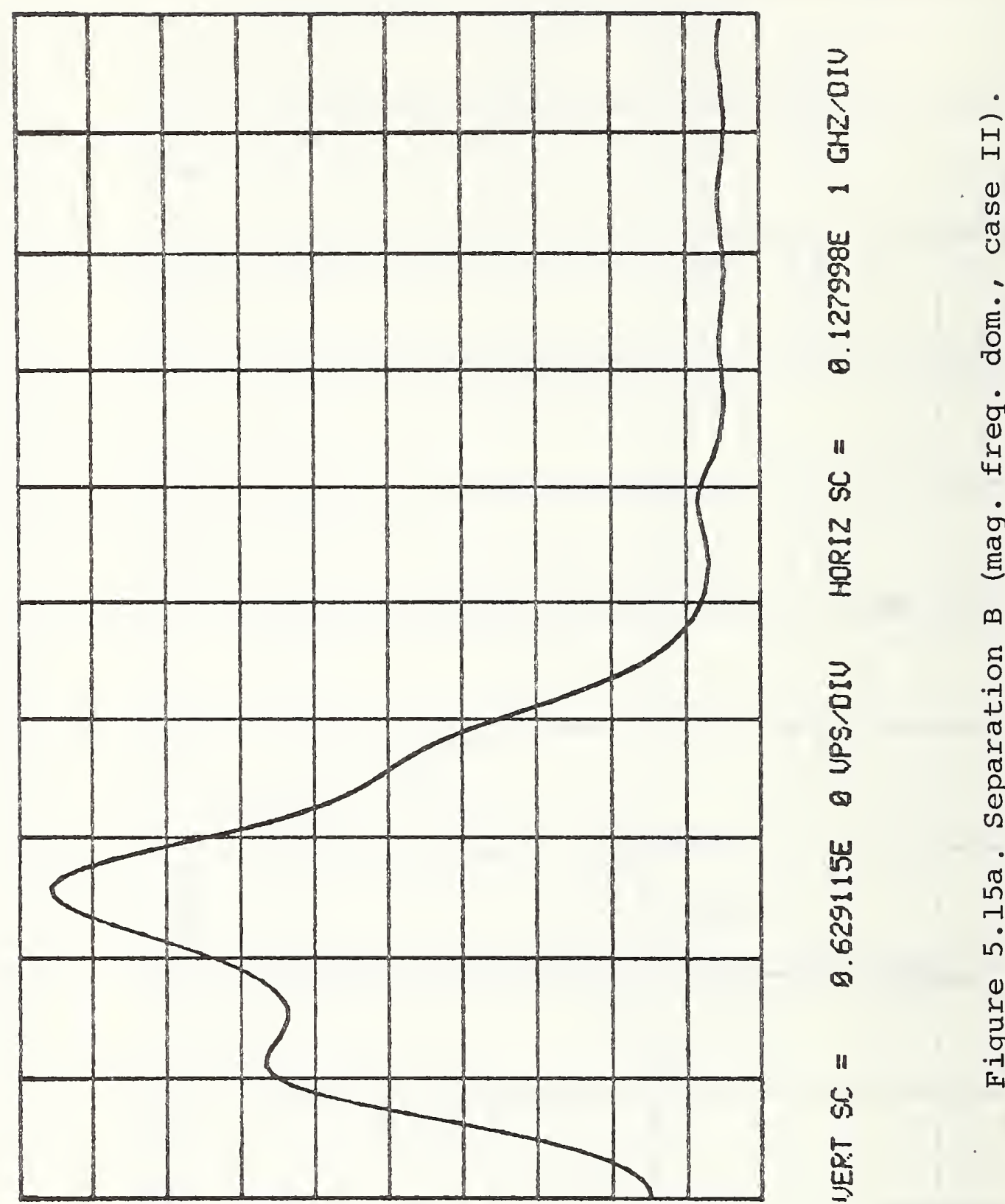

$\|$
0
0
$\frac{0}{2}$

نं

䆬

$m$

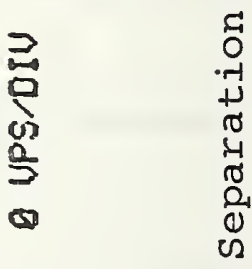

$\stackrel{w}{\stackrel{\omega}{D}}$

मी

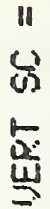




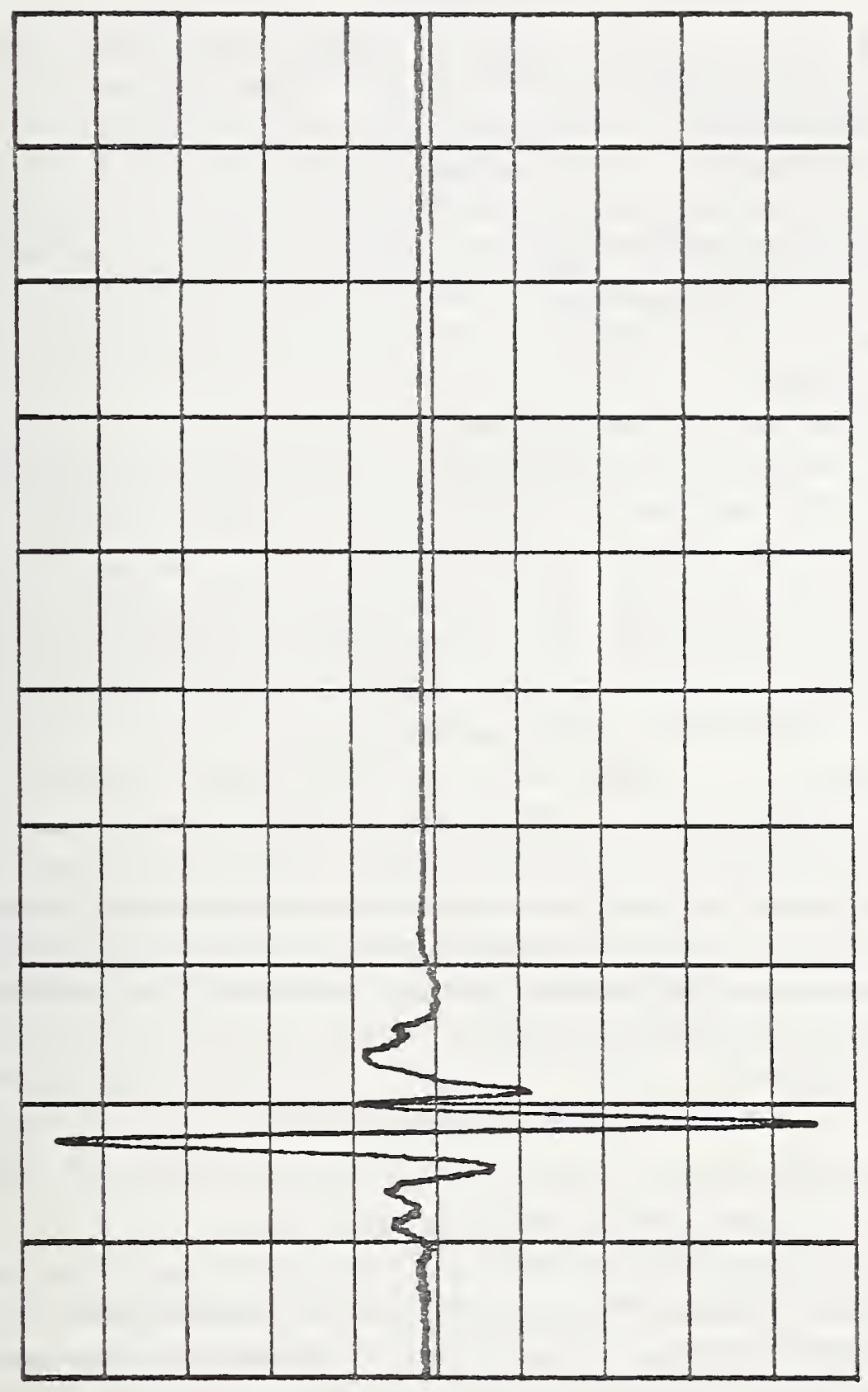

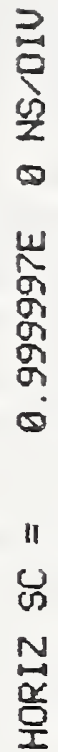

$\stackrel{-}{H}$

0
0
0
0

:

"

$m$

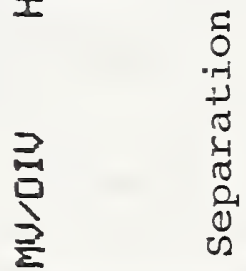

Y

盛

กำ

11
㟧 


\subsection{Introduction}

In chapters 3 and 4 it was noted that a given oscilloscope recording a time domain signal always presents a recorded waveform different from the signal waveform, which is due to the limited oscilloscope bandwidth. The recorded signal is the convolution of the signal waveform with that of the oscilloscope impulse response, figure 6.1. Also, it was shown in these chapters that the oscilloscope bandwidth limitation did not prevent the application of the homomorphic transformation to solve problems in which the oscilloscope transfer function was eliminated by insertion (ratio) measurements. However, if it is necessary to determine the true waveform at the input of the oscilloscope, then a model for the transfer properties of the oscilloscope is required.

In time domain spectroscopy, the overall bandwidth is determined by the product of the bandwidths of the signal source and the oscilloscope. In order to analyze the relative bandwidth contributions, it is necessary to determine a model for the oscilloscope.

For the picosecond time domain signals which are being dealt with in this work, the wideband sampling oscilloscope is used for observing and recording such signals. The one kind of sampling head that is most commonly used in time domain reflectometry measurements is the feed-through sampling-head because of the convenience of usage in observing incident and reflected time domain signals on the signal line, figure 6.2.

The aim of this chapter is to develop a network model based upon the physical properties of the sampling-head. Nominally, the manufacturer claims a 28 picosecond ( $10 \%$ to $90 \%$ ) transition time. The network model is to be used to predict both the oscilloscope's time and frequency domain responses. The predicted model responses are to be used to deconvolve the observed signals in order to deduce the true waveform at the oscilloscope input port. Previous workers have attempted to use heuristically based mathematical models which considered only an idealized sampling process [27].

\subsection{Theory and Construction of Sampler}

Previous publications $[28,29]$ went in depth into the theory and construction of the feed-through samplers; only a brief coverage will be presented here.

Figure 6.3 shows the balanced two-diode sampling circuit; this circuit is the basic structure in the feed-through sampler. The circuit works in the following manner. When the signal on the feed-through line is zero, application of a balanced strobing (sampling command) pulse on the two diodes will result in equal conduction in the diodes; consequently, an equal charge storage results on the sampling capacitors $\mathrm{C}_{\mathrm{S}}$ which in turn yields a zero output. But if the signal on the line has a nonzero value, nonequal diode conduction will result in a nonequal charge storage on the sampling capacitors and an output different from zero. The output polarity and magnitude are related to the input polarity and magnitude. The function of the feedback is to readjust the diodes' reverse bias after each sampling such that the next sampling process only detects the change (the difference) in the input 
signal. The feedback serves two purposes: (1) it increases the dynamic range for the allowable input voltage to the sampler, and (2) it maintains the operating region of the diode almost constant during each successive sample.

Since the output of the sampling circuit is a measure of the differences or level changes in the input signal, an integrating circuit is needed to give the required measure of the input signal. Figure 6.4 shows the dynamics of the sampling network assuming a ramp input signal and a triangular sampling pulse.

The detailed view of the physical realization of the two-diode feed-through sampler is shown in figure 6.5. In the figure it is seen that the feed-through transmission line is tapered or stepped-down on both the input and output sides; this is successively done through a three section compensated stepped-taper maintaining a 50 ohm impedance along the line (details shown in figure 6.8). The sampling diodes are put in a perpendicular fashion to the transmission line touching the inner conductor from both sides. In this region the inner conductor is a thin curved piece of metal that joins the two tapered sections together. The outer conductor forms a biconical cavity, which acts as a shorted biconical transmission line which shapes the strobe pulse, as shown in figure 6.6. The biconical cavity is dielectric filled to mask the packaging capacitance of the sampling diodes to lessen the effect of the diode on the $50 \mathrm{ohm}$ characteristic impedance throughout the cavity.

The sampling capacitors, 2 pf each, are built in the sampling diodes; figure 6.7 shows the details of a diode pack.

\subsection{Modeling of Physical Structure}

Precise measurements were performed on the physical dimensions of a disassembled sampling-head; the results are given in figure 6.8. Each of the step line discontinuities can be modeled by an equivalent capacitor [30,31]; the value of this capacitance is a function of the inner and outer diameters of the discontinuity. The discontinuity dimensions and capacitances are tabulated in Table 6.1. Figure 6.9 shows the equivalent circuit for the tapered structures complete with the characteristic impedances of line portions in between.

\subsection{Biconical Cavity}

The details of the cavity have been shown in figures $6.5,6.6$, and 6.8 . The cavity parameters of interest are (1) the characteristic impedance as seen by the signal along the feed-through line, (2) the characteristic impedance of the biconical transmission line as seen by the strobing pulse, (3) the travel time out and back along the shorted biconical line which determines the shape and duration of the sampling pulse applied to the two-diode sampling gate, figure 6.6, and (4) the characteristic impedance of the wire line carrying the strobing pulse down to the cavity. 
Table 6.1 .

\begin{tabular}{|c|c|c|c|c|c|}
\hline \multicolumn{2}{|c|}{ Discontinuity } & \multirow{2}{*}{$\frac{\text { Dimensions }}{c}$} & \multirow{2}{*}{$\frac{(\mathrm{mm})}{\mathrm{d}}$} & \multicolumn{2}{|c|}{ Capacitances ( $p f)$} \\
\hline$a$ & $b$ & & & $\mathrm{C}_{\text {in }}$ & $\mathrm{C}_{\text {out }}$ \\
\hline 6.9977 & 3.0429 & 4.9835 & 2.1636 & .00607 & .00884 \\
\hline 4.9835 & 2.1636 & 2.8575 & 1.2413 & .00803 & .01016 \\
\hline 2.8575 & 1.2412 & 2.0320 & 0.8890 & .00246 & .00349 \\
\hline 2.0320 & 0.8890 & & 0.6604 & .00170 & \\
\hline 2.0447 & 0.8814 & & 0.6604 & .00170 & \\
\hline 2.8448 & 1.2446 & 2.0447 & 0.8814 & .00246 & .00349 \\
\hline 4.9962 & 2.1654 & 2.8448 & 1.2446 & .00803 & .01016 \\
\hline 7.0104 & 3.0218 & 4.9962 & 2.1654 & .00607 & .00884 \\
\hline 7.0104 & 3.0218 & & 2.0244 & .00780 & \\
\hline 7.0104 & 3.0231 & & 2.0244 & .00780 & \\
\hline
\end{tabular}
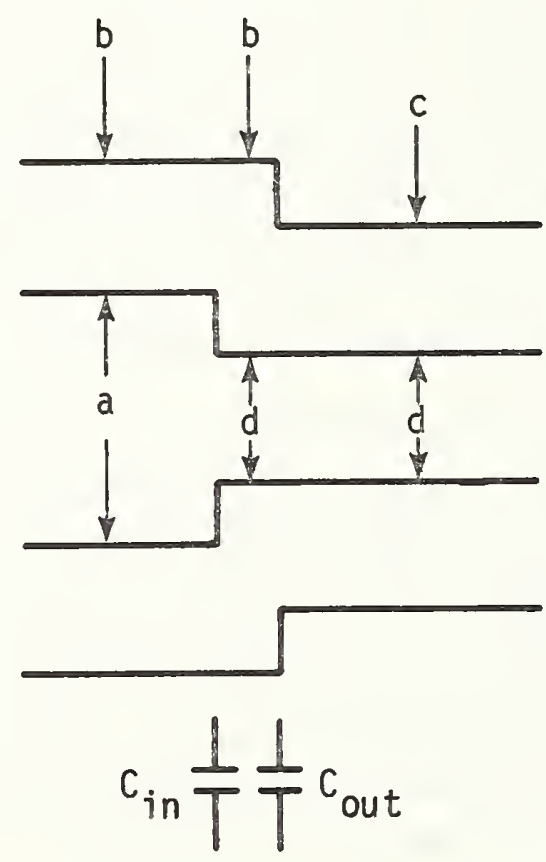
To find (1), (2), and (3) above, two TDR measurements were performed on the cavity with and without the dielectric filling, with the diodes removed in both cases. The results are shown in figure 6.10. Figure 6.10a clearly shows that the feed-through line is within \pm 1 ohm from the 50 ohm characteristic impedance; i.e., the reflected voltage is no greater than \pm 5 millivolts. Also, it is seen that there is an inductive effect in the central portion of the cavity due to the metallic curved strip that connects the tips of the two inner conductor tapered sections. From figure $6.10 \mathrm{~b}$, it is found that the absence of the dielectric filling caused a change in the impedance level that corresponds to a reflection coefficient of $\rho=0.196$, but since $\rho=(\sqrt{k}-1) /(\sqrt{k}+1)$, where $k$ is the dielectric constant, $k$ is obtained from $\sqrt{k}=(1+p) /(1-p)=1.49$ which gives

$$
k=2.21
$$

The value of the dielectric constant $k$ is to be used to calculate the travel time ' $b c$ ' figure 6.6. From

$$
\tau_{b c}=\sqrt{k} d_{b c} / c
$$

$d_{b c}$ is the corresponding travel distance which is equal to 4.48 mos aiven in figure 6.8 , and $c$ is the speed of light; therefore

$$
\tau_{b c}=1.49 \times 4.48 /\left(3 \times 10^{11}\right)=22.2 \text { ps. }
$$

The third cavity parameter to be determined is the characteristic impedance $\mathrm{z}_{\mathrm{bc}}$ of the "shorted" biconical line which can be calculated using the relation [32],

$$
z_{b c}=(120 \text { in } \cot (\alpha / 2)) / \sqrt{k} \text { ohms }
$$

where $\alpha$ is the bicone half angle which is equal to $38^{\circ} 22^{\prime}$ as given in figure 6.8 . The computed value of $z_{b c}$ is

$z_{b c}=85.2$ ohms.

Turning now to the wire line which carries the strobing pulse to the biconical cavity, the characteristic impedance $Z_{w}$ of the wire line is to be estimated. The inner conductor of the line has an outer diameter $r_{1}=0.127 \mathrm{~mm}$, coated with varnish to a diameter $r_{2}=0.33 \mathrm{~mm}$, while the inner diameter of the outside conductor is $r_{3}=0.432 \mathrm{~mm}$. An estimate for the value of the dielectric constant $k_{v}$ of the used varnish is $k_{v}=2$. The impedance $Z_{w}$ can be written as the sum of the two impedances $Z_{w 1}$ and $Z_{w 2}$.

$$
\begin{aligned}
z_{w} & =z_{w 1}+z_{w 2}=\frac{60}{\sqrt{k}} \ln \frac{r_{2}}{r_{1}}+60 \text { \&n } \frac{r_{3}}{r_{2}} \\
& \simeq \frac{60}{\sqrt{2}} \ln \frac{0.33}{0.127}+60 \ln \frac{0.43}{0.33} \\
& =57 \text { ohms. }
\end{aligned}
$$




\subsection{Sampling Diodes}

The sampling diodes that are being used in the feed-through sampler are hot carrier (Schottky) diodes; the electrical model of such diodes [33,34,35] is shown in figure 6.11, in which

$$
\begin{aligned}
& I_{s} \equiv \text { the diode (reverse) saturation current, } \\
& R_{j} \equiv \text { the junction's dynamic resistance, } \\
& C_{j} \equiv \text { the junction capacitance, } \\
& R_{s} \equiv \text { the spreading and contact resistance, } \\
& L_{d} \equiv \text { the lead inductance, and } \\
& C_{p} \equiv \text { the package capacitance, }
\end{aligned}
$$

where

$$
R_{j}=1 /\left(\frac{\partial I}{\partial V_{j}}\right)
$$

and

$$
I_{j}=I_{s}\left(\exp \left(V_{j} /\left(\frac{k T}{e}\right)\right)-1\right) .
$$

In the following, the measurements required for the evaluation of the above diode parameters are divided into two types: (1) static to evaluate $I_{S}$ and $R_{S}$ and (2) dynamic to evaluate $C_{p}, L_{d}$, and $C_{j}$.

\subsubsection{Static measurements ( $\mathrm{I}_{\mathrm{S}}$ and $\mathrm{R}_{\mathrm{S}}$ )}

The circuit of figure 6.12 was used to measure the diode's dc characteristics by taking values of the $d c$ current $I_{d}$ for various values of $d c$ diode voltage $V_{d}$; the measured values are plotted in figure 6.13. In this dc measurement, the diode currents and voltages are

$$
\begin{aligned}
& I_{j}=I_{d}, \\
& V_{j}=V_{d}-I_{j} R_{s} .
\end{aligned}
$$

In the forward bias case where $I_{j}>I_{s}$, the diode $I-V$ characteristic, eq. $(6.8)$, can be approximated as

$$
I_{j}=I_{s} \exp \left(V_{j} / \phi\right)
$$

or

$$
\ln I_{j}=\ln I_{s}+v_{j} / \phi ; \quad I_{j}>I_{s}
$$

where

$$
\phi=\mathrm{kT} / \mathrm{e} .
$$

Substituting eq. (6.9) in eq. (6.12) gives

$$
\ln I_{d}=\ln I_{s}+\left(V_{d}-I_{d} R_{s}\right) / \phi ; \quad I_{d} \gg I_{s} .
$$


The experimental data of figure 6.13 were fitted to eq. (6.13) above yielding the following values for $R_{s}$ and $I_{s}$ :

$$
\begin{aligned}
& R_{s}=19 \text { ohms } \\
& I_{s}=2.76 \times 10^{-11} \text { amps. }
\end{aligned}
$$

\subsubsection{Dynamic measurements $\left(C_{p}, L_{d}\right.$, and $\left.C_{j}\right)$}

To determine the sampling diodes' dynamic parameters $\left(C_{p}, L_{d}\right.$, and $C_{j}$ ), the TDR technique was used; the setup for the experiment is shown in figure 6.14. The test signal was the tunnel diode pulse given in figure 6.15. Because of the limited resolution due to the nonzero risetime of the test signal, the performed TDR measurements were not able to directly resolve the contributions of the sampling diodes from those of the central conductor inductance $L_{c}$; i.e., the TDR views the sampling diodes and the central inductance as if they were at the same physical location. This lumping can be tolerated (and later resolved) since the metallic strip extends for about $1 \mathrm{~mm}$ on both sides of the diodes in a symmetrical fashion. This symetry allows $L_{c}$ to be divided equally into two equal parts, $L_{L}$ and $L_{R}$ on the left and right of the diodes, respectively, figure 6.16 .

To overcome the TDR lumping of $\mathrm{L}_{c}$ and the diodes' contributions, two TDR measurements were performed; one with both diodes in place, and the other one with only one of the diodes in place, figures $6.17 \mathrm{a}$ and $6.17 \mathrm{~b}$. Denoting the maximum voltage drop-peaks as $e_{1}$ and $e_{2}$, respectively, the TDR voltages are

$$
\begin{aligned}
& e_{1}=2 e_{d}+e_{L} \\
& e_{2}=e_{d}+e_{L}
\end{aligned}
$$

where $e_{L}$ and $e_{d}$ are the contributions in the TDR signal corresponding to $L_{c}$ and diode, respectively. Solving eqs. $(6.16)$ and $(6.17)$ for $e_{L}$ and $e_{d}$ yields

$$
\begin{aligned}
& e_{L}=2 e_{2}-e_{1} \\
& e_{d}=e_{1}-e_{2} .
\end{aligned}
$$

Substituting for $e_{1}$ and $e_{2}$ from figure 6.17 gives

$$
\begin{aligned}
& e_{L}=-9.9 \mathrm{mV} \text { (drop-peak) } \equiv 9.9 \mathrm{mV} \text { peak } \\
& e_{d}=19.7 \mathrm{mV} .
\end{aligned}
$$

The value of eq. $(6.20)$ can be used to evaluate the inductance $L_{c}$ using the relation [36]

$$
L=\frac{2 z_{0}}{m} e_{L}
$$

where $m$ is the maximum slope of the test pulse, figure 6.15. Therefore,

$$
\mathrm{L}_{c}=\frac{2 \times 50}{6.58} \times 9.9 \times 10^{-3}=150 \mathrm{ph}
$$


and

$$
L_{L}=L_{R}=75 \mathrm{ph} \text {. }
$$

To measure the package capacitance $C_{p}$ of the diode, a third TDR measurement, figure $6.17 \mathrm{c}$, was done with one working diode and an open diode; the open diode contributes only $C_{p}$. Denoting the voltage drop-peak in this measurement $e_{3}$, the TDR voltage relation is

$$
e_{3}=e_{d}+e_{p}+e_{L}
$$

where $e_{p}$ is the contribution of the open diode, or in other words, the $c_{p}$ contribution. Consequently,

$$
e_{p}=e_{3}-e_{d}-e_{L}
$$

Upon substituting for $e_{3}$ from figure 6.17c, and for $e_{L}$ and $e_{d}$ using eqs. (6.20) and (6.21), the value of $e_{p}$ is given by

$$
e_{p}=20.8-19.7+9.9=11 \mathrm{mV} \text {. }
$$

$C_{p}$ can then be calculated using [36]

$$
\mathrm{c}=\frac{2}{\mathrm{mZ}} \mathrm{e}_{\mathrm{c}}
$$

which yields

$$
C_{p}=\frac{2}{6.58 \times 50} \times 11=.067 \mathrm{pf}
$$

For the order of magnitudes involved in the present TDR measurement, computations showed that the effect of diode inductance $L_{d}$ on the voltage drop-peaks is minor; i.e., such measurements are not able to yield the value $L_{d}$. However, previous measurements [37] that were done on the same particular sampler gave a complex value $14+j 5$ ohms at $10 \mathrm{GHz}$ for the impedance $\mathrm{z}_{\mathrm{s}} / 2$ of the sampling gate with a diode biasing forward current of $5 \mathrm{ma}$. This information can be used to calculate the value of $\mathrm{L}_{\mathrm{d}}$ as follows: the measured impedance at the sampling gate is in fact the impedance of the parallel combination of the two sampling diodes, each one in series with its sampling capacitor $\mathrm{C}_{S}$; this combination is shown in figure 6.18. At 5 ma diode forward current, the junction resistance is

$$
\begin{aligned}
& R_{j}=1 /\left(\frac{\partial I}{\partial V_{j}}\right)=\phi / I_{j}=5.2 \Omega \\
& R_{j} / 2=2.6 \Omega .
\end{aligned}
$$

Since $\mathrm{C}_{j}$ is expected to be lower than $0.1 \mathrm{pf}$, the reactance of $2 \mathrm{C}_{j}$ at $10 \mathrm{GHz}$ is higher than $80 \Omega$, which is much greater than $R_{j} / 2$; the reactance of $2 C_{j}$ can be neglected in this 
particular case. Hence, the impedances can be expressed as

$$
\frac{z_{s}}{2}=\frac{1}{j \omega 2 C_{s}}+\frac{\left(1 / j \omega 2 C_{p}\right) \cdot\left(R_{s} / 2+R_{j} / 2+j \omega L_{d} / 2\right)}{\left(1 / j \omega 2 C_{p}\right)+\left(R_{s} / 2+R_{j} / 2+j \omega L_{d} / 2\right)} .
$$

Since $C_{p}, R_{s}$, and $R_{j}$ are known, eq. (6.32) above can be solved to give

$L_{\mathrm{d}}=300 \mathrm{ph}$.

Now, the only component that is left to be determined is $\mathrm{C}_{j}$; and since it is known to be a function of the junction voltage, a set of TDR measurements, with both diodes in place, was performed at different biasing voltages. The biasing circuit was that shown in figure 6.19 , and in figure 6.20 only two of the various TDR waveforms are shown; however, all the results are listed in table 6.2. Denoting the voltage drop-peak in these TDR runs by $e(v)$, where $\mathrm{v}$ is the diode bias, the TDR voltage is

$$
e(v)=2 e_{p}+2 e_{j}(v)+e_{L}
$$

from which $e_{j}(v)$, the contribution due to $c_{j}$, can be obtained as

$$
e_{j}(v)=\left(e(v)-e_{L}-2 e_{p}\right) / 2 \text {. }
$$

The corresponding $C_{j}(v)$ is

$$
c_{j}(v)=\frac{2}{m Z_{0}} e_{j}(v) \text {. }
$$

These steps are shown also in table 6.2 , and the calculated $c_{j}(v)$ is plotted in figure 6.21 . By evaluating $C_{j}(v)$, this phase of modeling the sampling diodes is concluded.

In summary the obtained values for the diode model, figure 6.11 , are

$$
\begin{aligned}
& C_{p}=0.067 \mathrm{pf} \\
& L_{d}=300 \mathrm{ph} \\
& R_{s}=19 \Omega \\
& I_{s}=2.76 \times 10^{-11} \mathrm{amp} \\
& C_{j}(v) \text { is given in figure } 6.21 .
\end{aligned}
$$

Also, the value of the central conductor inductances has been determined as

$$
\mathrm{L}_{\mathrm{L}}=\mathrm{L}_{\mathrm{R}}=75 \mathrm{ph} \text {. }
$$

\subsection{The Sampling Pulse}

The experimental setup of figure 6.22 was used to observe the strobe pulse waveform; only the leading edge of the pulse will be considered since it is the only part of the waveform that forms the sampling pulse, figure 6.6. The observed signal is shown in figure 6.23 which shows an amplitude of 9.58 volts and a $10 \%$ to $90 \%$ risetime $\tau_{\mathrm{m}}$ of $53.5 \mathrm{ps}$; both of these values have to be corrected as explained in the following discussion. 
Table 6.2.

\begin{tabular}{llll}
\hline$v v$ & $e(v) m V$ & $e_{j}(v) m V$ & $c_{j}(v) p f$ \\
0.0 & 26.86 & 7.40 & .045 \\
-0.25 & 24.90 & 6.42 & .039 \\
-0.50 & 23.58 & 5.76 & .035 \\
-0.75 & 23.26 & 5.60 & .034 \\
-1.00 & 22.60 & 5.27 & .032 \\
-1.25 & 21.94 & 4.94 & .030 \\
-1.50 & 21.60 & 4.77 & .029 \\
-1.75 & 21.28 & 4.61 & .028 \\
-2.00 & 20.62 & 4.28 & .026 \\
-2.25 & 20.63 & 4.28 & .026 \\
-2.50 & 20.28 & 4.11 & .025 \\
-2.75 & 19.96 & 3.95 & .024 \\
-3.00 & 19.62 & 3.78 & .023 \\
\hline
\end{tabular}


The characteristic impedances, $\mathrm{Z}_{\mathrm{o} 1}, \mathrm{Z}_{\mathrm{o} 2}$, and $\mathrm{Z}_{\mathrm{o} 3}$, of the stripline (on which the strobe pulse was measured), the (wire) microminiature coaxial line connecting the stripline to the biconical cavity, and the biconical line are 50, 57, and 85.2 ohms, respectively, by virtue of eqs. (6.3) and (6.4). These unmatched line impedances cause a voltage amplification factor A given by

$$
A=\left(1+\rho_{1}\right)\left(1+\rho_{2}\right)
$$

where the reflection coefficients $\rho_{1}$ and $\rho_{2}$ are determined as

$$
\begin{aligned}
& \rho_{1}=\frac{z_{o 2}-z_{o 1}}{z_{o 2}+z_{o 1}}=\frac{57-50}{57+50}=.065 \\
& \rho_{2}=\frac{z_{o 3}-z_{o 2}}{z_{o 3}+z_{o 2}}=\frac{85.2-57}{85.2+57}=0.198
\end{aligned}
$$

from which $A$ is calculated as

$$
A=(1+.065) \cdot(1+0.198)=1.276
$$

The amplitude of the observed strobe pulse vs. time has to be multiplied by the factor 1.276 to yield the corresponding amplitude at the input of the biconical shorted line.

The risetime correction has to take into consideration the attenuators, adaptors, and measuring sampling-head responses. Experiments [38] have shown that elements have the following nominal risetimes:

$$
\begin{aligned}
& 20 \text { ps for } 10 \mathrm{~dB} \text { attenuator, } \\
& 18 \text { ps for } 20 \mathrm{~dB} \text { attenuator, } \\
& 10 \text { ps for each adaptor, and } \\
& 20 \text { ps for measuring sampling-head. }
\end{aligned}
$$

Using the risetime square rule for the cascaded setup of figure 6.22, the equivalent risetime $\tau_{\mathrm{e}}$ of this combination is nominally

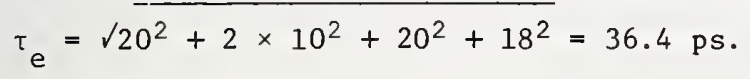

Using the square rule again, the estimated risetime $\tau_{s}$ of the strobe pulse is

$$
\tau_{s}=\sqrt{\tau_{m}^{2}-\tau_{e}^{2}}=\sqrt{53.5^{2}-36.4^{2}}=39.2 \mathrm{ps} .
$$

An assumed response having 36.4 ps risetime could be used to deconvolve the observed strobe pulse to yield an estimate of the leading edge of the actual strobe pulse waveform. Another method, which was found to be as good, is to scale the time variable in the observed waveform by the ratio $\tau_{s} / \tau_{m}$ to yield an estimate for the actual waveform. The two methods gave values which were within $5 \%$ of each other.

The corrected leading edge waveform for the strobe pulse at the input port of the shorted biconical line is given in figure 6.24 . 


\subsection{Structure Step Response}

In sections 6.2 through 6.6 all the sampling-head components were modeled. The complete model (equivalent circuit) would be the same as that shown in figure 6.9 except for the cavity box to be replaced by its model, figure 6.25 .

To study the step response of the sampler, the computer program TDNA given in appendix C is to be used; a voltage step is to be applied to the input port, and the sampled output is to be integrated to yield the step response. As seen from figures 6.9 and 6.25 , the number of elements in the model is quite large; consequently, the amount of computer memory required to consider this model was beyond the capacity of a large computer. The network (model) of figures 6.9 and 6.25 was divided into some independent networks, in which case the step response can be obtained in two steps: the structure step response and the sampling process. Figure 6.26 shows the simplified network considered in the first step. Here the short lines of figure 6.9 and the discontinuity capacitors on each side are replaced by one lumped capacitor, and two lines on both sides were matched in impedance to their neighboring lines in impedance. In this way the number of lines and capacitors are reduced to about one half. Also, in the figure the sampling signal and feedback voltage are ignored; the diode bias is set to a constant negative value $v_{b}=-1.63$ volts, at which the actual sampling diodes normally operate, as was experimentally measured.

The network parameters shown in figure 6.26 were given to the computer as input data for the program TDNA. The obtained time domain signal $v_{8}$ at the node 8 is shown in figure 6.27 .

\subsection{Sampling Process}

The second, and final, step in obtaining the sampling-head step response is to sample the resultant voltage waveform of node 8 using the computer program SMPL given in appendix $C$. The voltage signal $\mathrm{v}_{8}$ is to be applied to node 17 in figure 6.28 , each time the balanced strobing signal is applied to nodes 2 and 16 . The reverse bias of the diodes $V_{b}$ is kept at -1.63 volts, the normal operation value. The difference between the resultant voltage increments on the sampling capacitors $C_{s 1}$ and $C_{s 2}$ is a measure of the output voltage $V_{\text {out }}$. The sampling efficiency $n[27,28]$ is defined as

$$
\eta=\frac{v_{\text {out }}}{v_{\text {in }}} ; v_{\text {in }} \equiv v_{8} \text {. }
$$

As mentioned earlier in section 6.2, the feedback voltage readjusts the diodes' reverse bias after each sample such that the next sampling process only detects the change in the input signal; consequently, at the beginning of the sampling interval, the net diode bias is a constant -1.63 volts. Accordingly, and referring to figure 6.28 , the value of $V_{f b}$ for a new sampling process should be equal to $v_{\text {in }}$ of the previous sampling;

$$
\mathrm{v}_{\mathrm{fb}}=\mathrm{v}_{\mathrm{in}}
$$


Figure 6.29 shows a block diagram of the sampling network and its feedback from the figure, and using eqs. $(6.45)$ and $(6.46)$ it can be written that

$$
\begin{aligned}
V_{f b} & =\beta G V_{\text {out }} \\
& =\beta G \eta V_{\text {in }} \\
& =v_{\text {in }} ;
\end{aligned}
$$

therefore,

$$
\beta G \eta=1
$$

or

$$
B G=\frac{1}{\eta}=\frac{v_{\text {in }}}{v_{\text {out }}} .
$$

To determine the factor BG, a preliminary run for the computer program SMPL was done using a small voltage step at node 17 as an input, and upon calculating its ratio to the output voltage, the obtained values were

$$
\begin{aligned}
V_{\text {in }} & =.01 \text { volt } \\
V_{\text {out }} & =.0026907 \text { volts } \\
B G & =\frac{V_{\text {in }}}{V_{\text {out }}}=\frac{.01}{.0026907}=3.7165 .
\end{aligned}
$$

The determination of the factor $\beta G$ allows the calculation of the feedback voltage $V_{\text {fb }}$ after each sample using eq. $(6,47)$.

The progran SIPL was run using the voltage $V_{8}$ as the input signal to the sampling network, and the input signal was sampled at 1 ps time intervals. The obtained output was normalized to yield the required sampling-head step response, figure 6.30. The step response shows a 30.5 ps transition time.

Figures 6.31 through 6.34 show the voltage waveforms of the sampling process for one sampling interval. Figure 6.31 shows the time domain waveform of the resultant sampling pulse $v_{3-1}$ (voltage difference between nodes 3 and 1 in figure 6.28). The voltage across one of the diodes $V_{6-9}$ is shown in figure 6.32, while the voltage across one of the sampling capacitors $\mathrm{V}_{3-6}$ is shown in figure 6.33. The difference between the two sampling capacitor voltages $\mathrm{V}_{12-1}-\mathrm{V}_{3-6}$ is the sampled-data output, figure 6.34. It is seen that the resultant shape of the sampling pulse is somewhat different from being triangular, and the sampling time $\tau_{s}$ (the time of diode conduction) is about $21 \mathrm{ps}$.

In sumary, it has been shown that the waveform of the sampling process or "sampling pulse" is derived from the strobe pulse step like transition through shaping by the biconical cavity into a quasi-triangular pulse which is in turn shaped by the nonlinear and bandlimiting properties of the sampling diode pair. 


\subsection{The Effect of the Diode Bias on the Sampling-Head Step Response}

For ideal sampling, the sampling pulses should be infinitesimal in duration; a diracdelta function is the ideal shape. The actual sampling pulse, figure 6.31 , does have nonzero duration; consequently, the step response of the sampling-head is expected to have a nonideal (nonzero) transition time. From the theory of sampling processes it is clear that the greater the duration of the sampling pulse, the slower will be the step response transition time.

There is one way for the operator to control the sampling pulse duration and, consequently, the step response; this is accomplished by controlling the diodes reverse bias, thus varying the level at which the diodes conduct [39], figure 6.35. The larger the diode reverse bias, the smaller will be the sampling time; consequently, the faster will be the response of the sampling-head, and vice versa. However, there are limits to bias variations; very low or very high reverse bias will cause imperfect sampling. Figure 6.36 shows the observed waveforms of a tunnel diode step using the feed-through sampling-head for three values of diode reverse bias corresponding to (a) bias for optimum sampling, (b) low reverse bias, and (c) high reverse bias.

Additional tunnel diode waveforms were measured for various values of the diode reverse bias. The $10 \%-90 \%$ transition time $\tau_{r}$ was calculated for each waveform. The obtained $\tau_{r}$ was plotted vs. reverse bias as in figure 6.37. Since the tunnel diode transition time is constant, the observed variations are due to the sampling-head response variations. The values of the sampling time $\tau_{s}$ for various values of reverse bias were computed (using the program SMPL) and also plotted in figure 6.37. The figure agrees with the fact that the smaller the sampling time the faster the response of the sampling-head, within the limits of effective feedback control on the sampling level voltage. For reverse bias values higher than 1.76 volts, the response is slower unlike the theoretical expectations. From the figure it is clear that the fastest response for the sampling-head can be obtained by adjusting the diode reverse bias to the value of 1.76 volts; the observed waveform at this bias was slightly different from the normal sampling case (1.63 volts bias which results from the manufacturer's alignment procedure for proper operation).

The sampling-head step response for the fastest response bias (1.76 volts) was computed and is given in figure 6.38, where it is seen that the step response shows a 27.5 ps transition time. Figure 6.39 gives the corresponding impulse response (time derivative).

\subsection{The Deconvolution Routine}

The final step in the present chapter is to develop a deconvolution routine that uses the sampling-head impulse response given in figure 6.39 for deconvolving an observed signal in order to deduce the true waveform at the sampling-head input port. In other words, and referring to figure 6.1 , the problem is to find $x(t)$ knowing both $y(t)$ and $h(t)$.

The simplest deconvolution method known is to calculate the ratio of the frequency domain transforms $Y\left(e^{j \omega}\right) / H\left(e^{j \omega}\right)$ to obtain $X\left(e^{j \omega}\right)$, the frequency domain form of $x(t)$. This method inherently produces a very noisy output, because in the division process the zeros 
and the poles of $\mathrm{H}\left(\mathrm{e}^{\mathrm{j} \omega}\right)$ must be exactly cancelled out. Any errors in the data representing $\mathrm{Y}\left(\mathrm{e}^{j \omega}\right)$ and $\mathrm{H}\left(\mathrm{e}^{j \omega}\right)$ degrade the cancellation and introduce rapid variations (noise) in the resultant $X\left(e^{j \omega}\right)$. In order to suppress the noise, low pass filtering and/or pole removal processes have to be applied, the latter acting on poles introduced by incomplete pole/zero cancellations. The pole removal processes are very sensitive which makes the use of the simple ratio method for deconvolution rather unlikely.

Although more complicated deconvolution methods are available for use, they a11 involve successive approximations to an estimated input $x(t)$ to minimize the error between the estimated output and the actual output $y(t)$.

In this section a slightly modified homomorphic deconvolution method will be used successfully to deconvolve the output $y(t)$ to obtain $x(t)$. The method is advantageous in its simplicity as well as yielding a low level of output noise in $x(t)$.

6.10.1 The homomorphic deconvolution method

In the homomorphic deconvolution as was developed in chapter 2, the cepstrum of a convolution is the linear sum of the cesptras of the individual components of this convolution. That is, using the notation of chapter 2 as applied to the signals of figure 6.1 , it can be written that

$$
\hat{y}(\hat{t})=\hat{x}(\hat{t})+\hat{h}(\hat{t}) .
$$

Knowing both $y(t)$ and $h(t)$, the cepstras $\hat{y}(\hat{t})$ and $\hat{h}(\hat{t})$ can be used to determine $\hat{x}(\hat{t})$ through the simple subtraction

$$
\hat{x}(\hat{t})=\hat{y}(\hat{t})-\hat{h}(\hat{t}) \text {. }
$$

The resultant $\hat{x}(\hat{t})$ can be processed (inverse-homomorphically transformed) back to the time domain to yield the required $x(t)$.

\subsection{0 .2 An application}

To show the capability of the homomorphic deconvolution method as presented above, the method will be applied to a known convolution. This known convolution $y(t)$ was formed by convolving the sampling-head impulse response $h(t)$, figure 6.39 , and an assumed typical step-like time domain signal $x_{a}(t)$, figure 6.40 . The resultant convolution $y(t)$ is shown in figure 6.41. The cesptras $\hat{y}(\hat{t})$ and $\hat{h}(\hat{t})$ given in figures 6.42 and 6.43 , respectively, were obtained by applying the homomorphic transformation to their corresponding time domain forms. The difference $\hat{x}_{d}(\hat{t})=\hat{y}(\hat{t})-\hat{h}(\hat{t})$ is shown in figure 6.44. Applying the inverse homomorphic transform to $x_{d}(t)$ yields $x_{d}(t)$, the deconvolved estimate for the assumed input signal $x_{a}(t)$. Comparison of figures 6.45 and 6.40 shows that $x_{d}(t)$ matches to a great extent the original signal $x_{a}(t)$ except for a very small amount of error oscillations which are due to sampling and computational errors. 


\subsection{Summary and Conclusions}

The present chapter was devoted to the modeling of the feed-through sampling-head. The theory and construction of the sampling head were reviewed. The physical dimensions of the structure discontinuities were used to formulate an equivalent network for the samplinghead input and output lines. The dimensions of the biconical cavity together with TDR measurements were used to model the cavity part of the sampler. The dc characteristics of the diode together with TDR measurements vs. sampling diode bias were used to evaluate various diode model parameters. The strobing pulse was measured, and the data corrected for both amplitude and risetime.

All of these sampling-head parameters were simulated in the form of a digital computer program which was run to obtain a theoretical estimate of the sampling-head step response. The program was implemented in two steps because of the large number of elements and information required to simulate the sampling system.

The step response was found to vary with the diode reverse bias; the higher the reverse bias the faster was the step response, within limits. The step response at the manufacturer suggested bias and at the bias for the fastest step response was obtained.

The homomorphic deconvolution transform developed in chapter 2 was slightly modified in order to be used to deconvolve the sampling-head response contribution from the output of the sampling oscilloscope. Deconvolution of the observed waveform provides an estimate of the actual (true) waveform at the input port of the oscilloscope. 


$$
\begin{aligned}
\begin{array}{c}
\text { SIGNAL OF } \\
\text { INTEREST }
\end{array} & \begin{array}{c}
\text { OSCILLOSCOPE } \\
H\left(e^{j \omega}\right) \\
h(t)
\end{array} \\
y(t)=x(t) * h(t) & \begin{array}{c}
\text { OBSERVED } \\
\text { (RECORDED) } \\
\text { SIGNAL }
\end{array} \\
Y\left(e^{j \omega}\right) & =x\left(e^{j \omega}\right) \cdot H\left(e^{j \omega}\right)
\end{aligned}
$$

Figure 6.1 Oscilloscope Response Effect on Measured Signals.

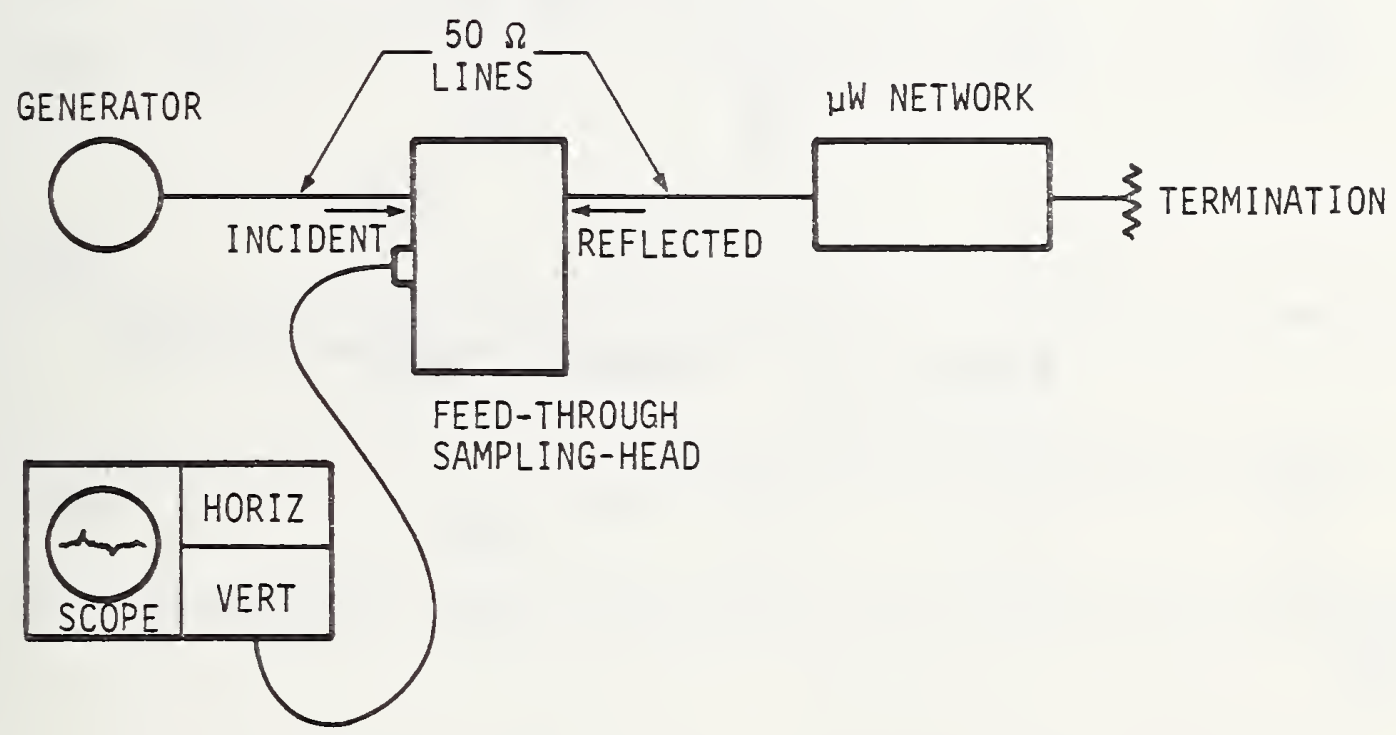

Figure 6.2. Feed-Through Sampling Oscilloscope as Used in TDR Measurements. 


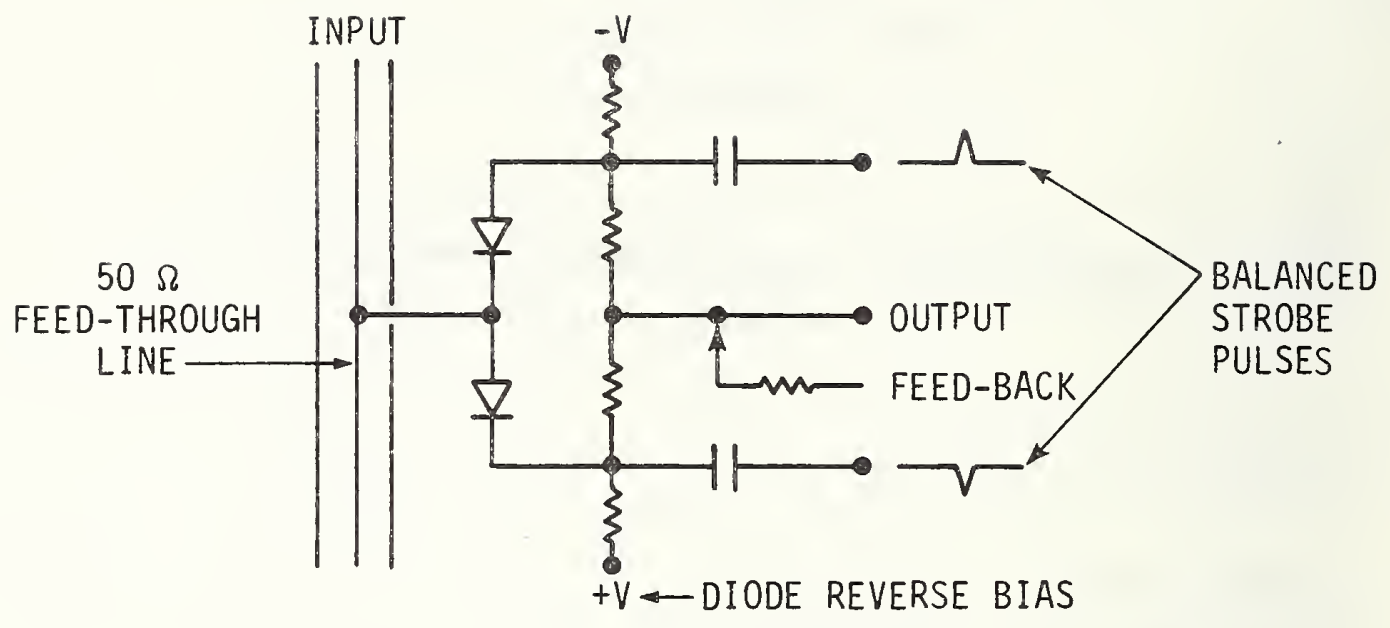

Figure 6.3. Two-Diode Sampling Circuit. 

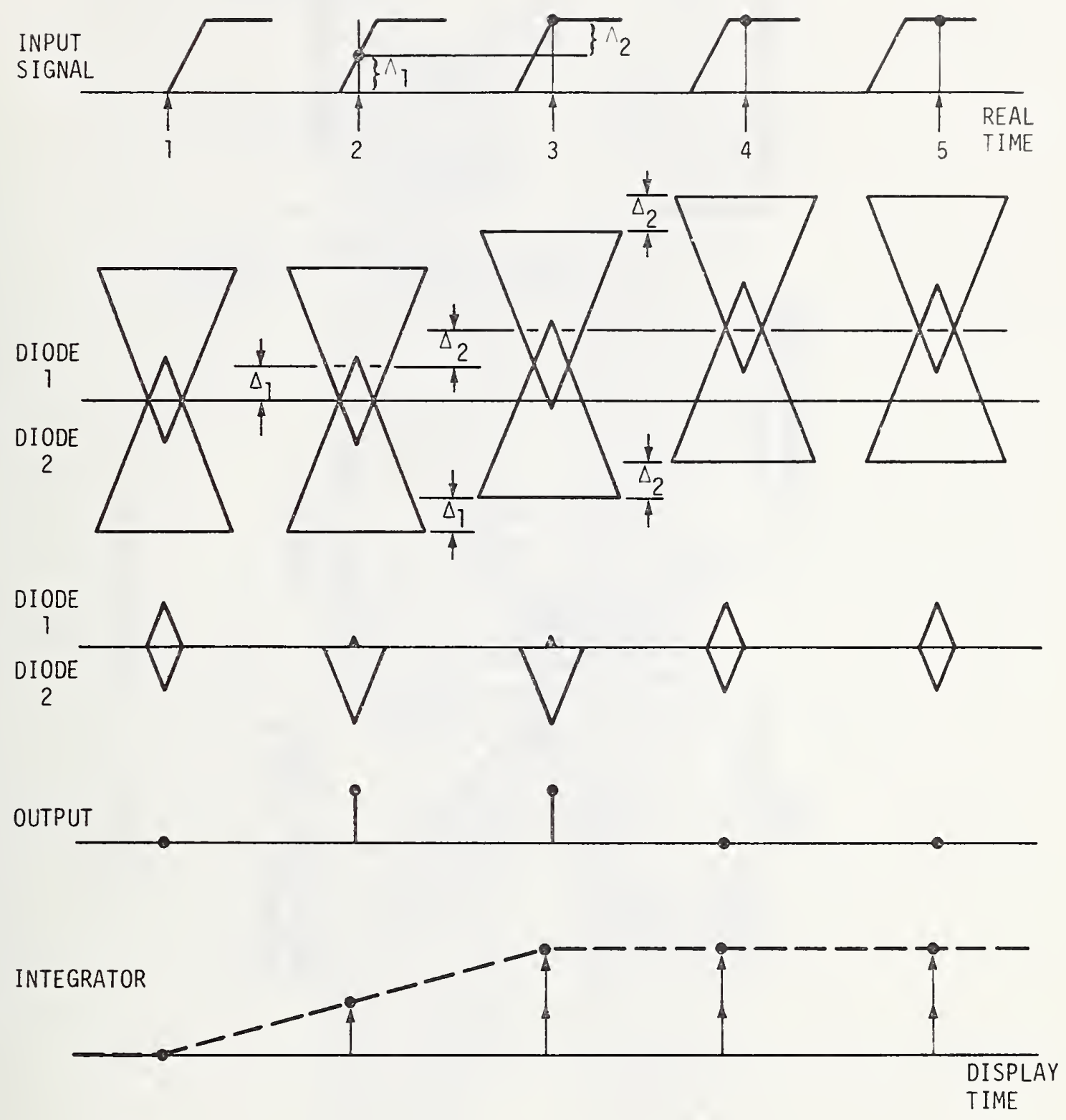

Figure 6.4. Sampling Process. 


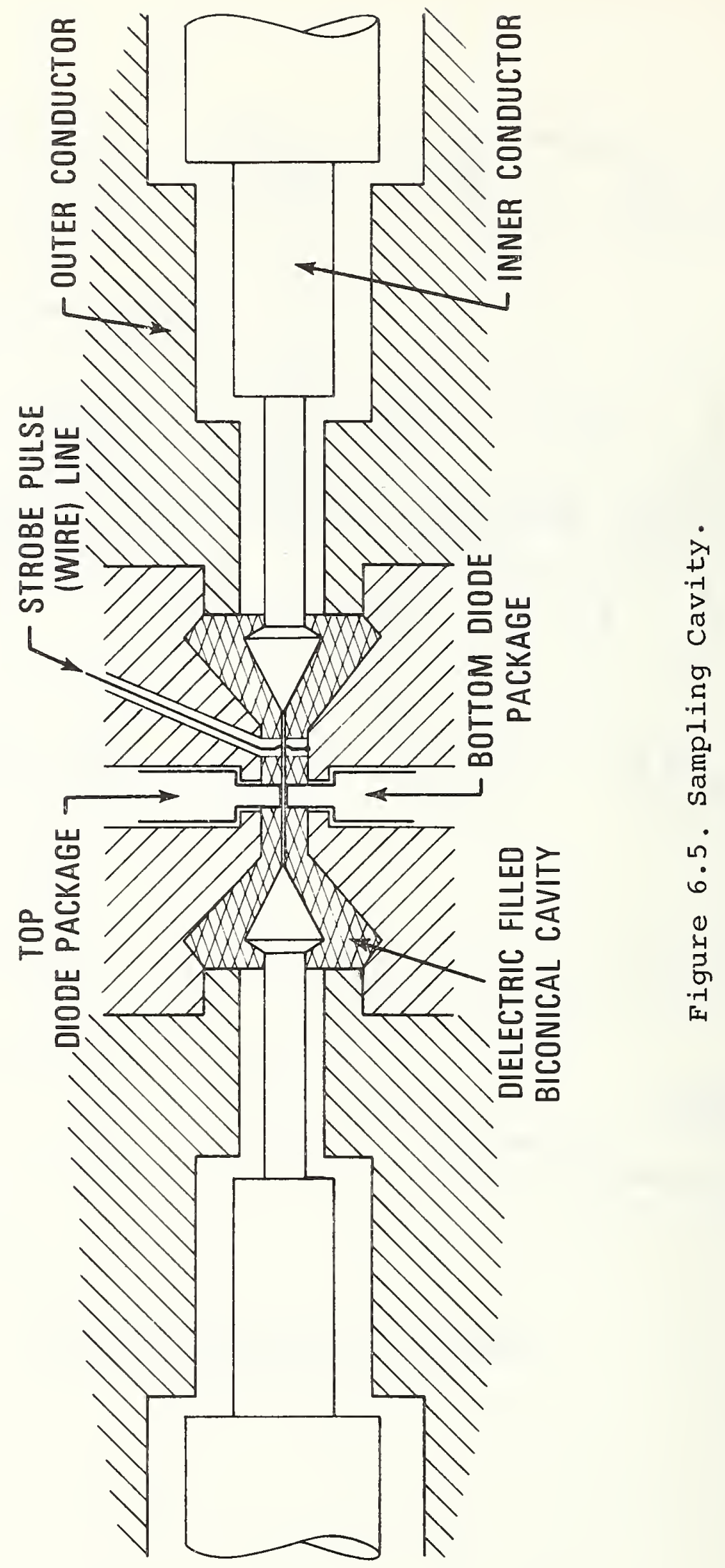



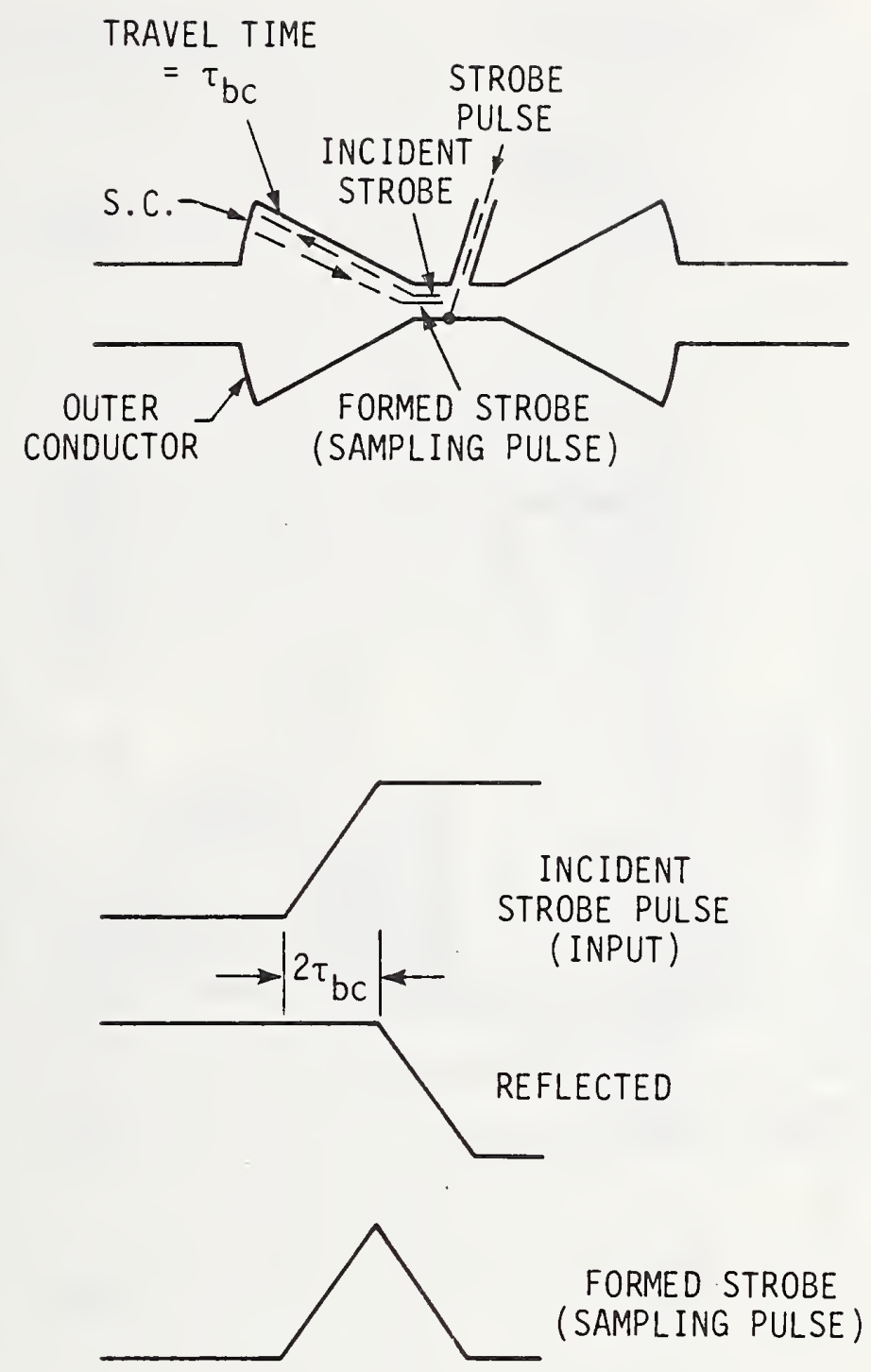

Figure 6.6. Strobe Pulse Shaping. 


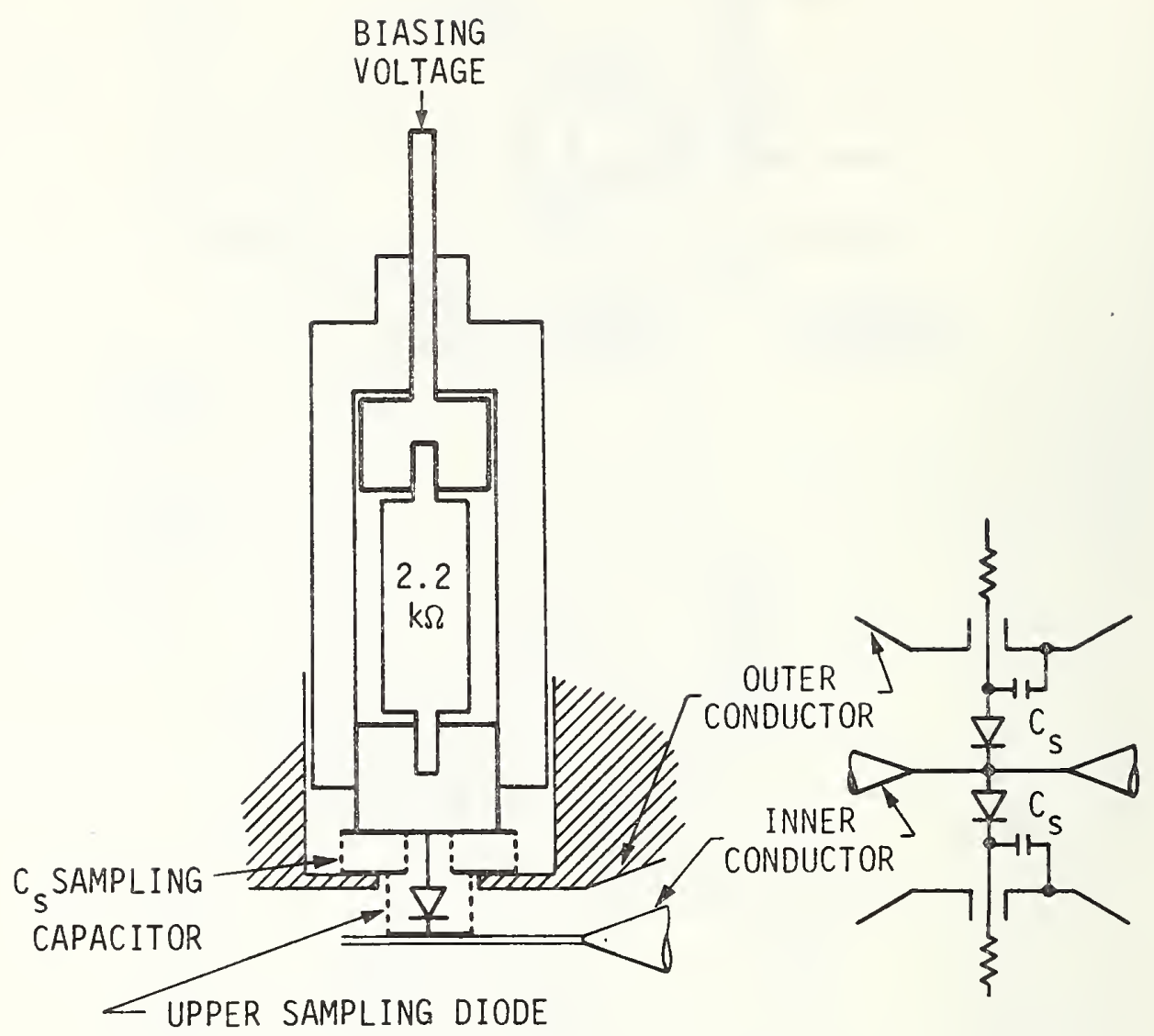

Figure 6.7. Diode Pack. 


$\frac{\operatorname{INNER} \text { DIAMETERS }(\mathrm{mm})}{\operatorname{LENGTHS}(\mathrm{mm})}$

43.4416

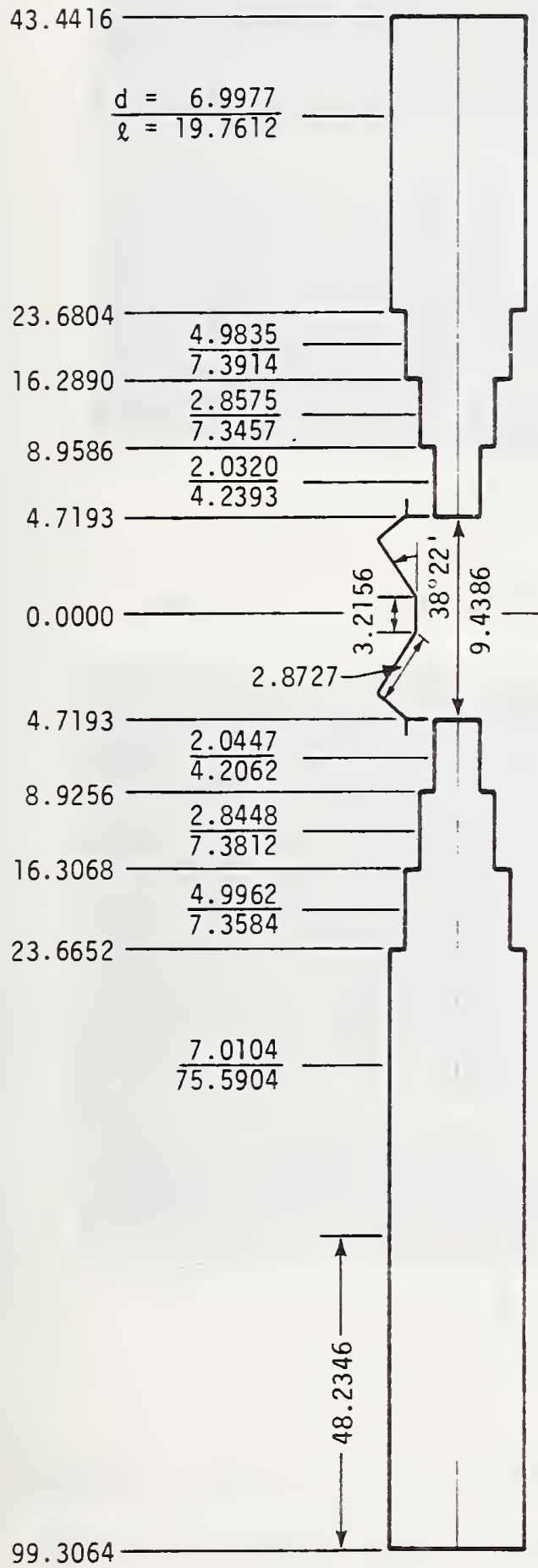

OUTER DIAMETERS (mm)

LENGTHS $(\mathrm{mm})$

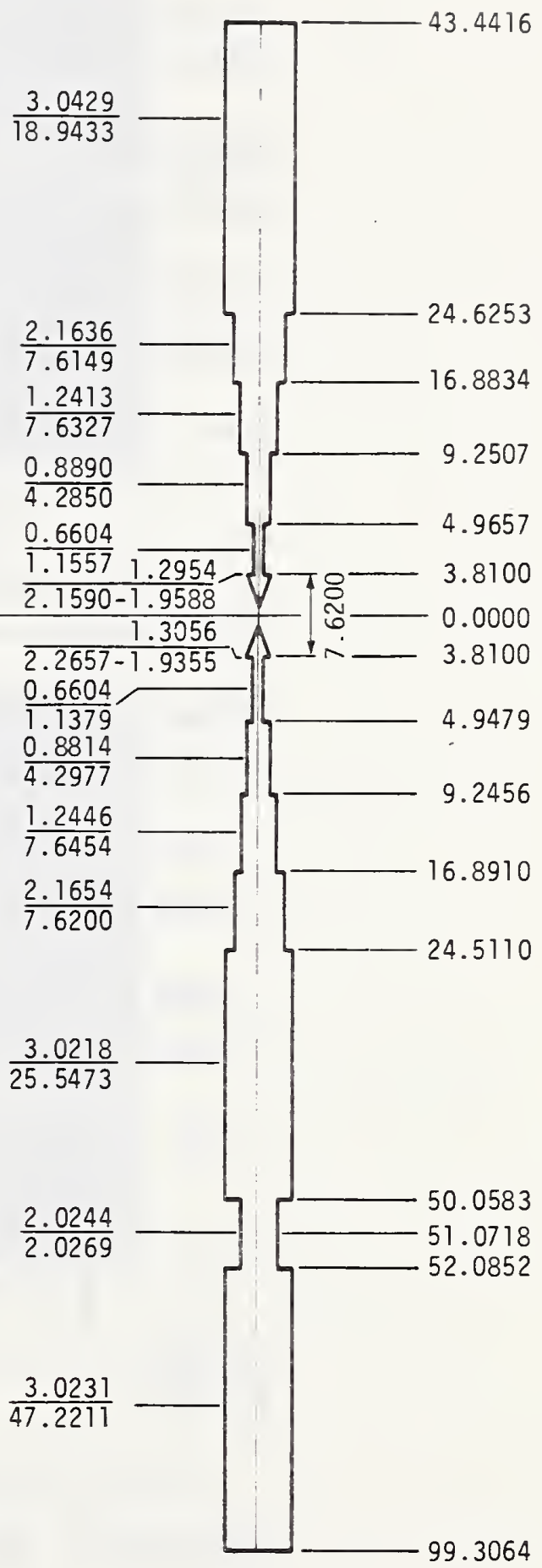

Figure 6.8. Sampling Head Dimensions. 


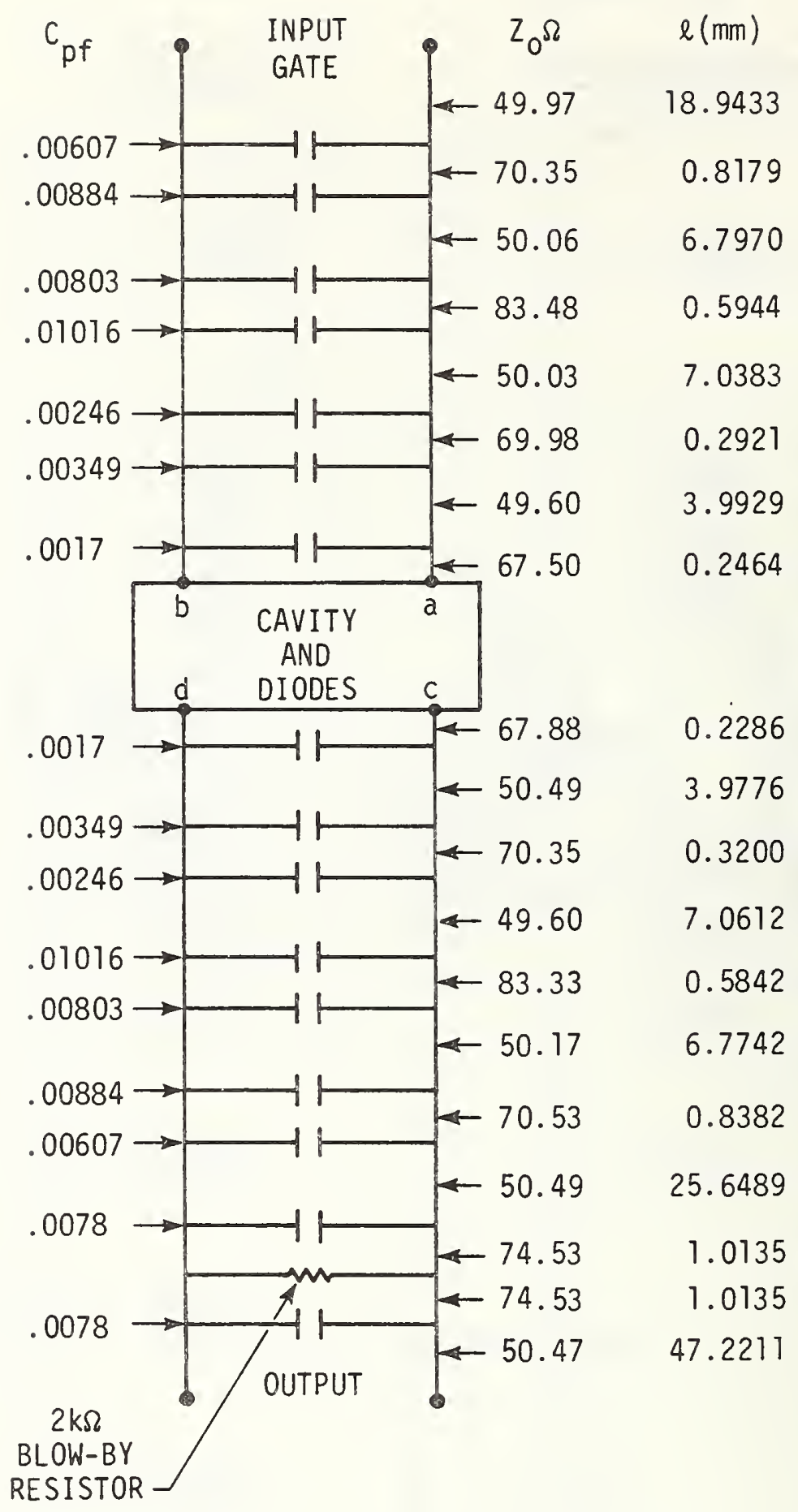

Figure 6.9. Tapered Line Equivalent Circuit. 


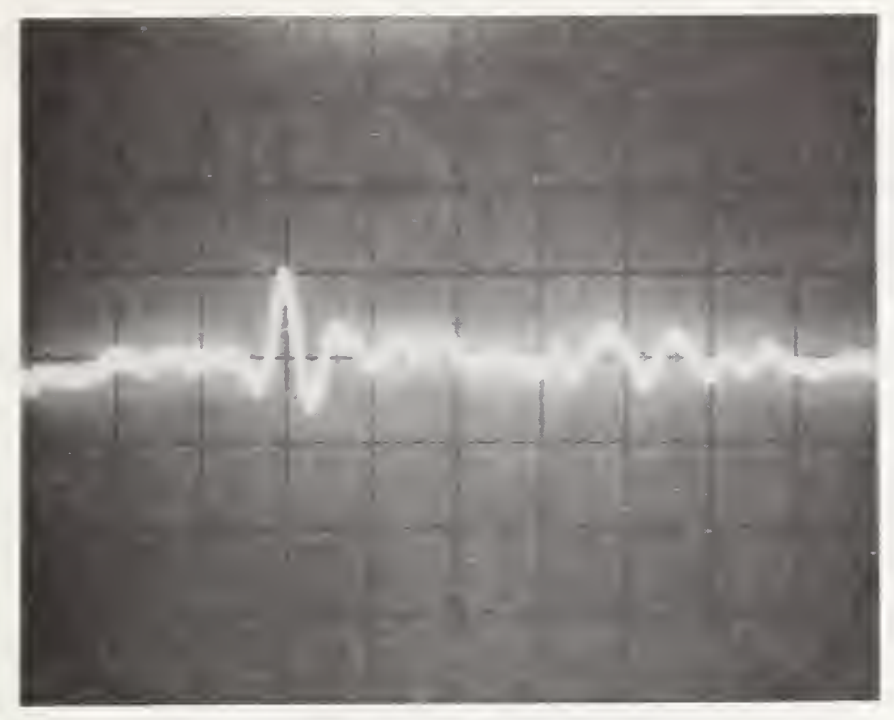

a. With Dielectric.

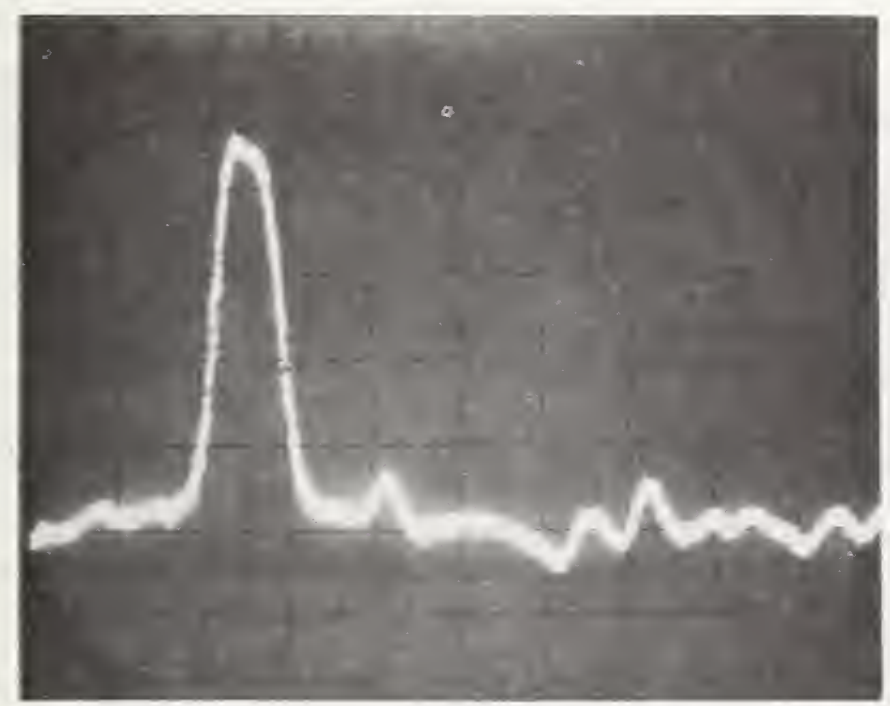

b. With no Dielectric.

Figure 6.10. TDR on Cavity with no Diodes. vert. $10 \mathrm{mV} / \mathrm{div}(0=.0425 / \mathrm{div})$ Horiz. $100 \mathrm{ps} / \mathrm{div}$ 


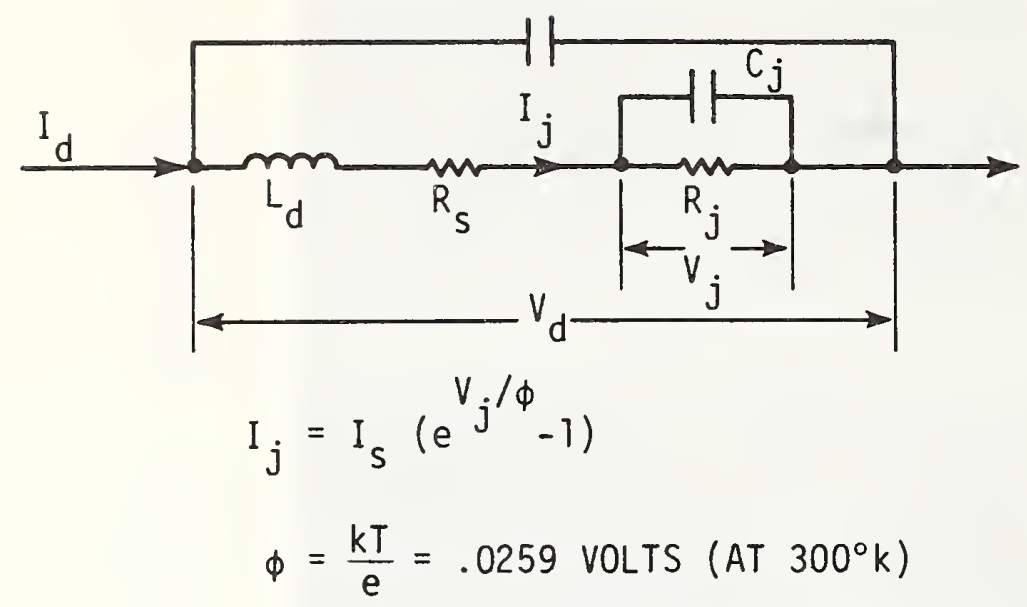

Figure 6.11. Diode Electrical Model.

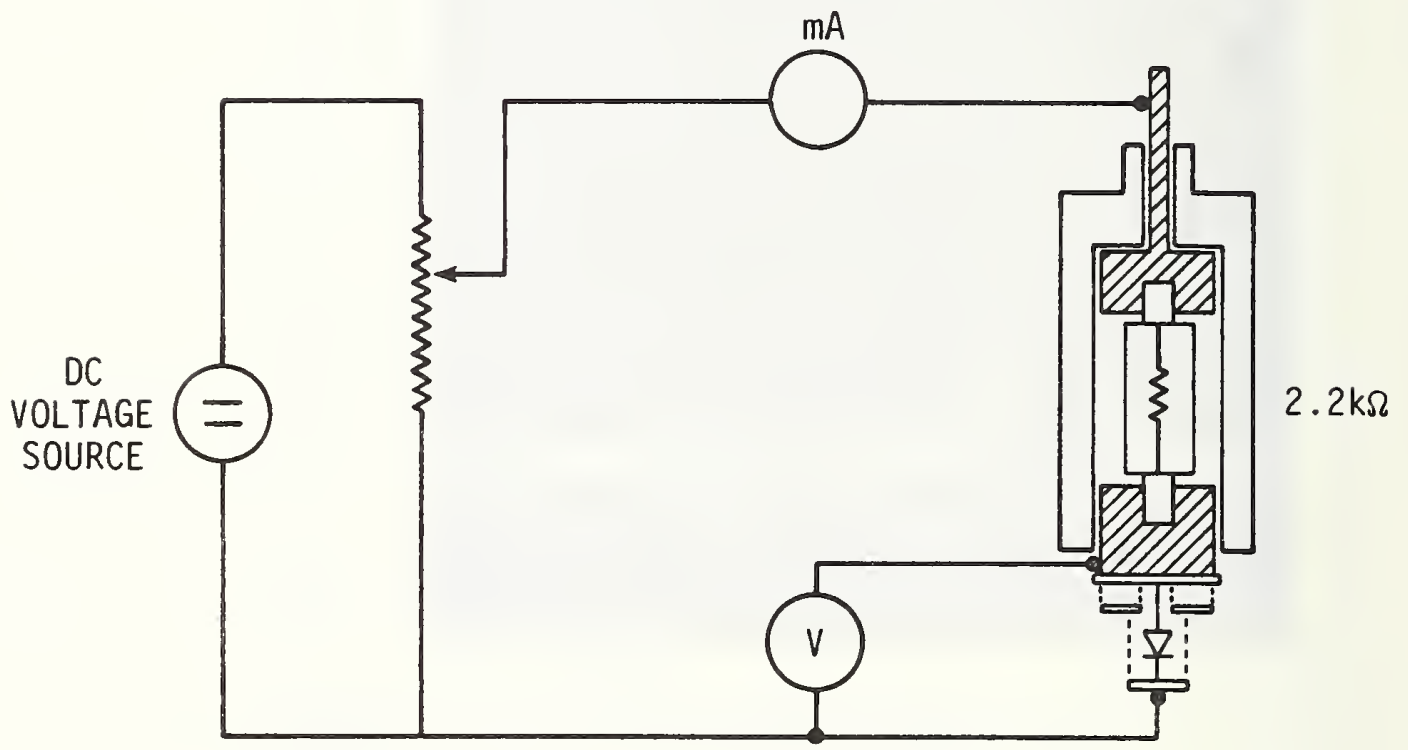

Figure 6.12. Circuit Used to Measure Static Characteristics of a Sampling Diode. 


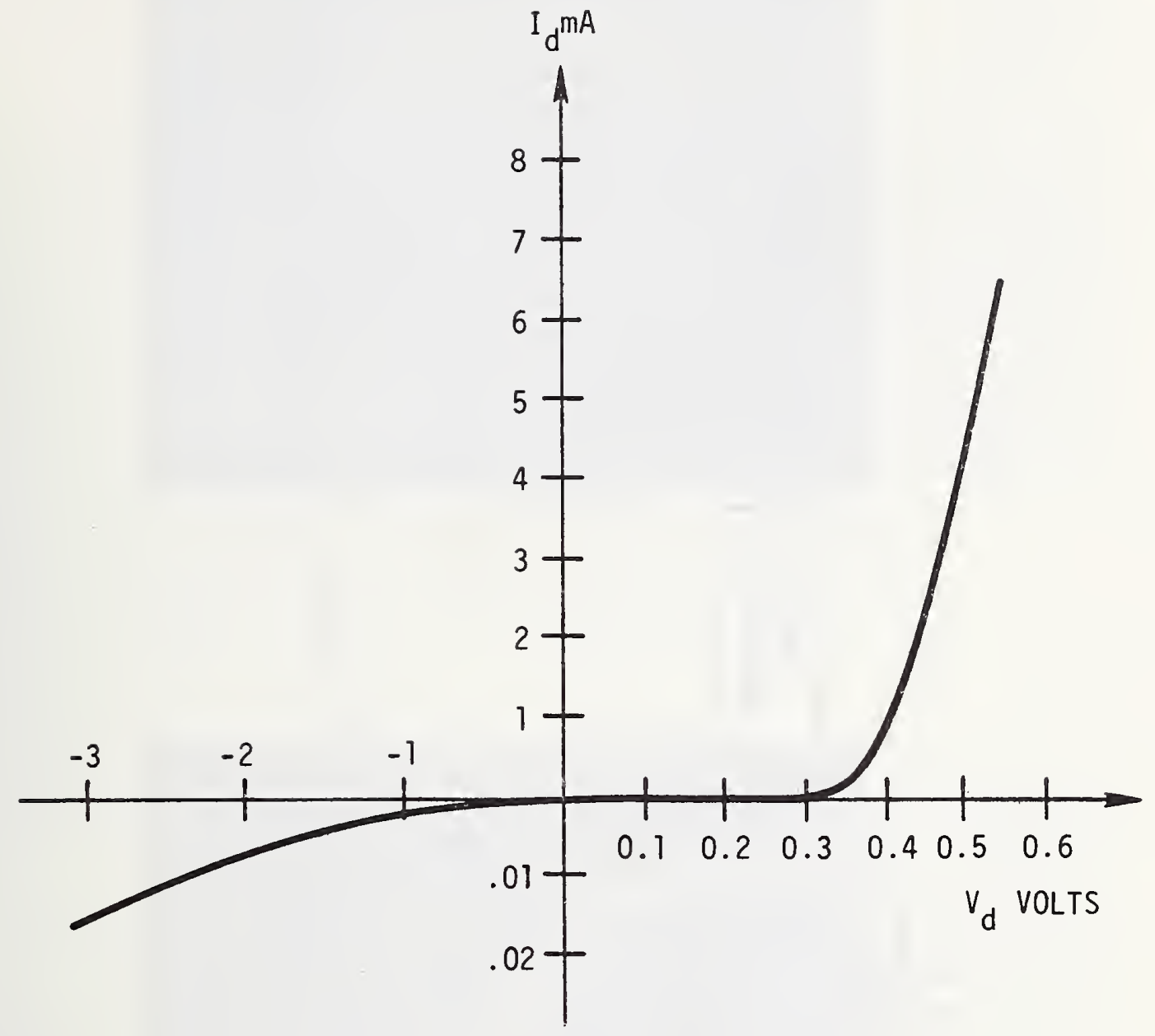

Figure 6.13. Didoe Static Characteristics. 


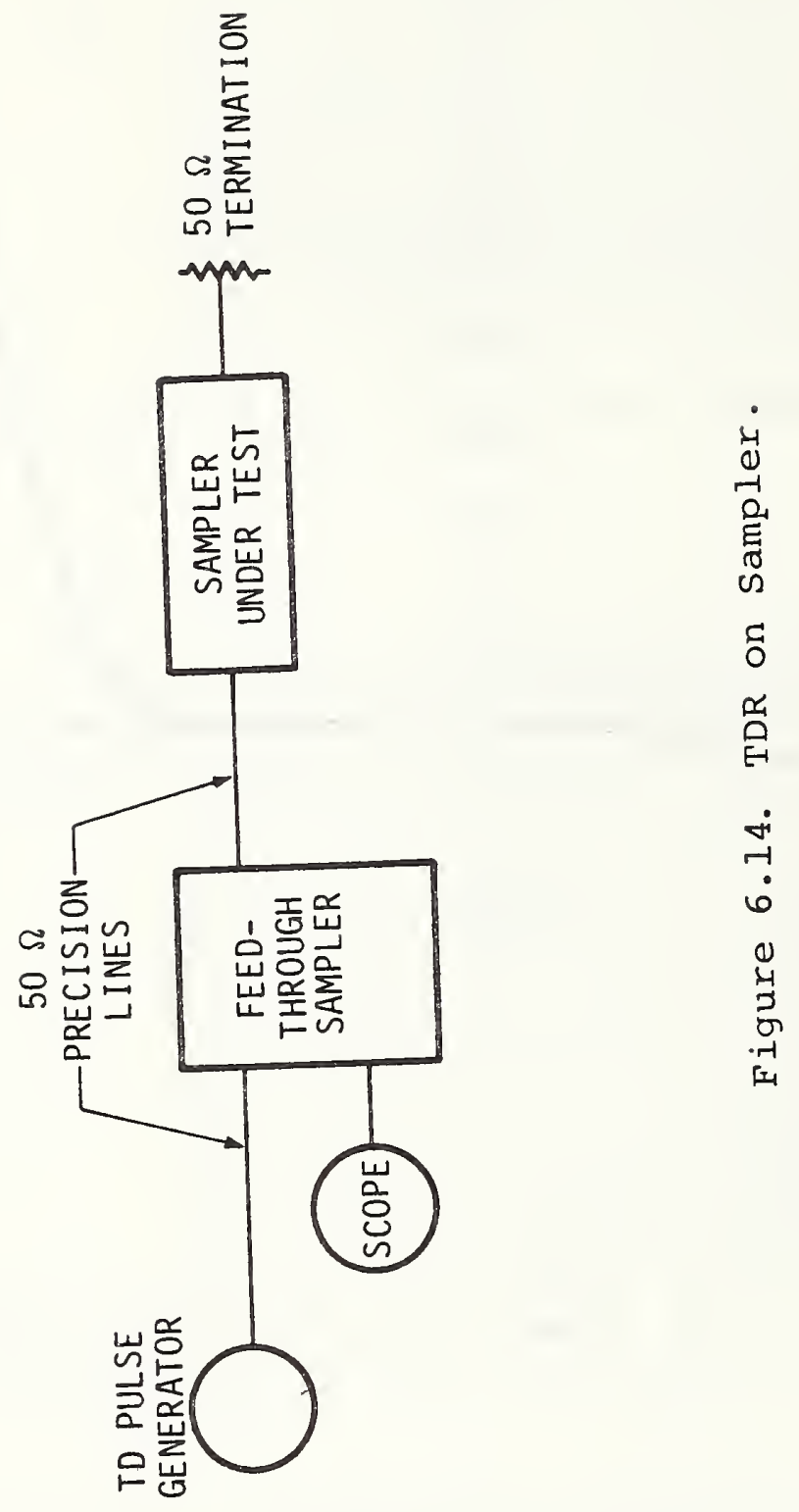




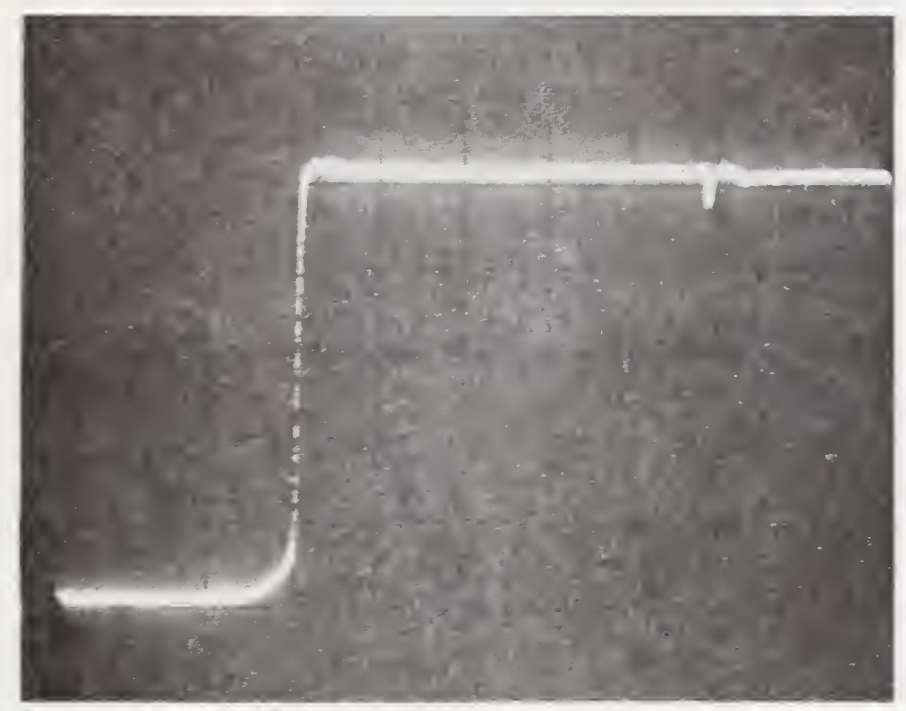

a. Horiz. $1 \mathrm{~ns} / \mathrm{div}$, Vert. $50 \mathrm{mv} / \mathrm{div}$.

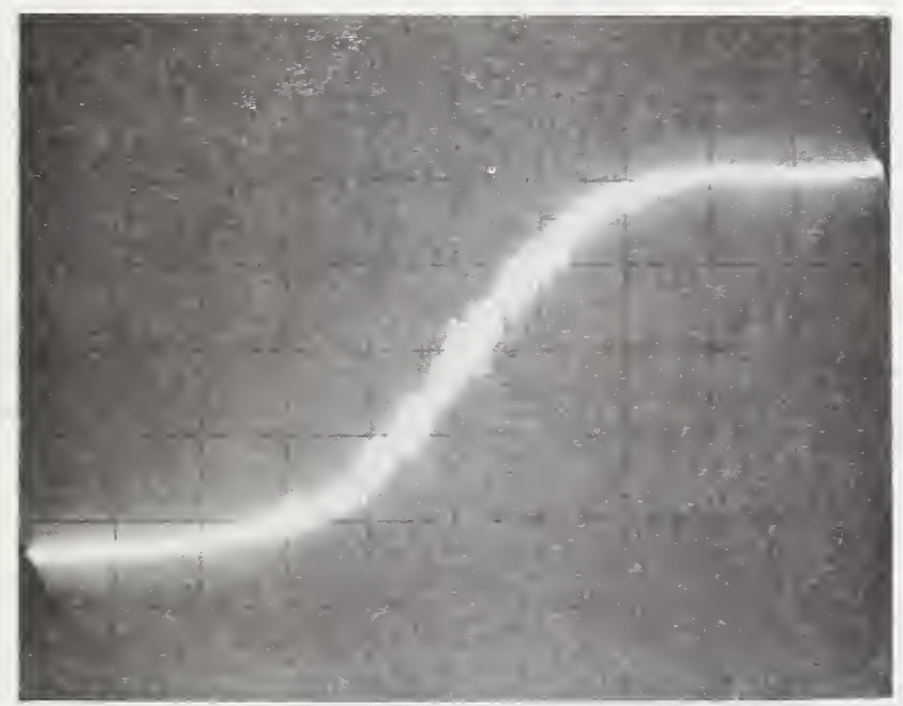

b. Horiz. $10 \mathrm{ps} /$ div, Vert. $50 \mathrm{mv} / \mathrm{div}$.

Figure 6.15. TD Pulse. 

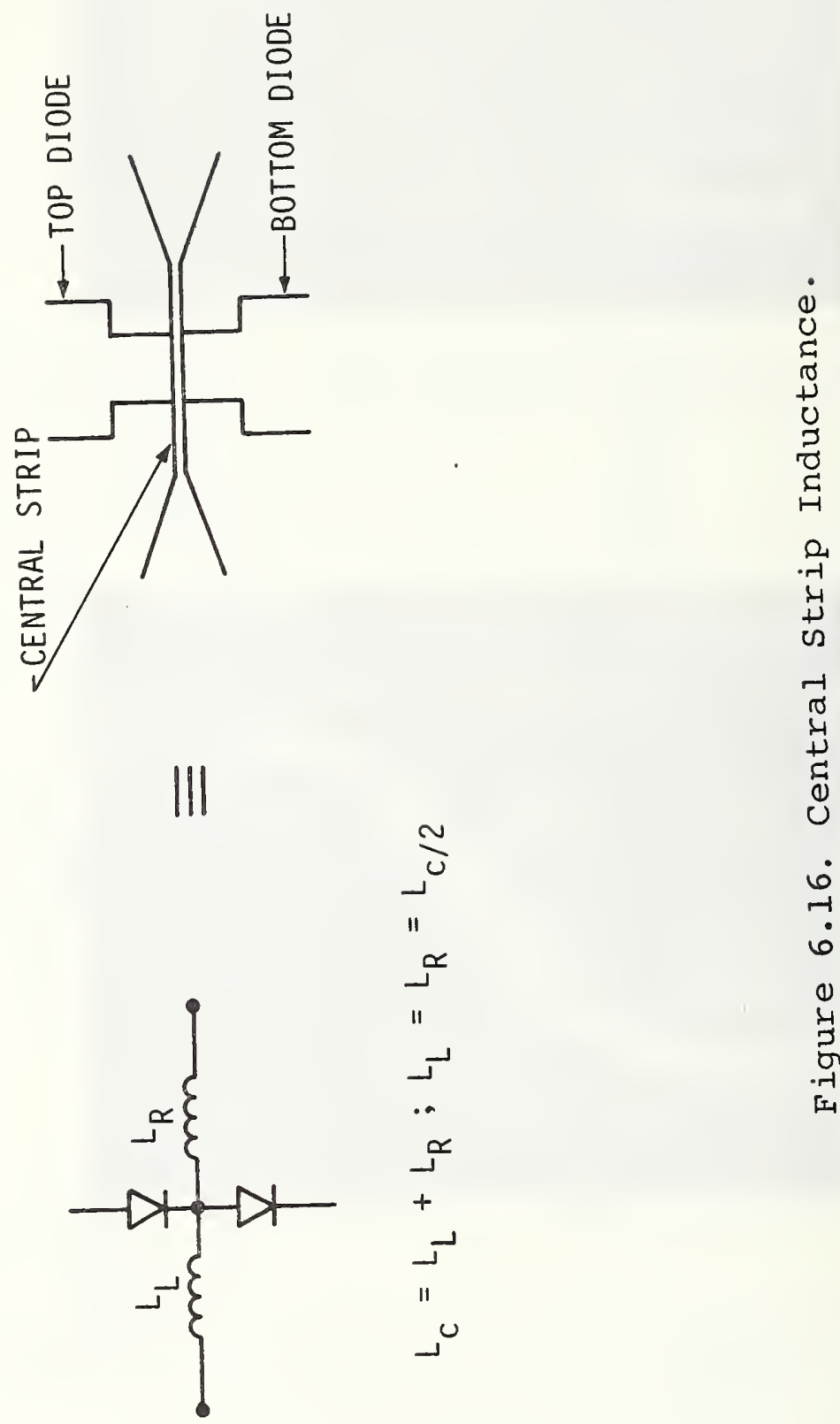

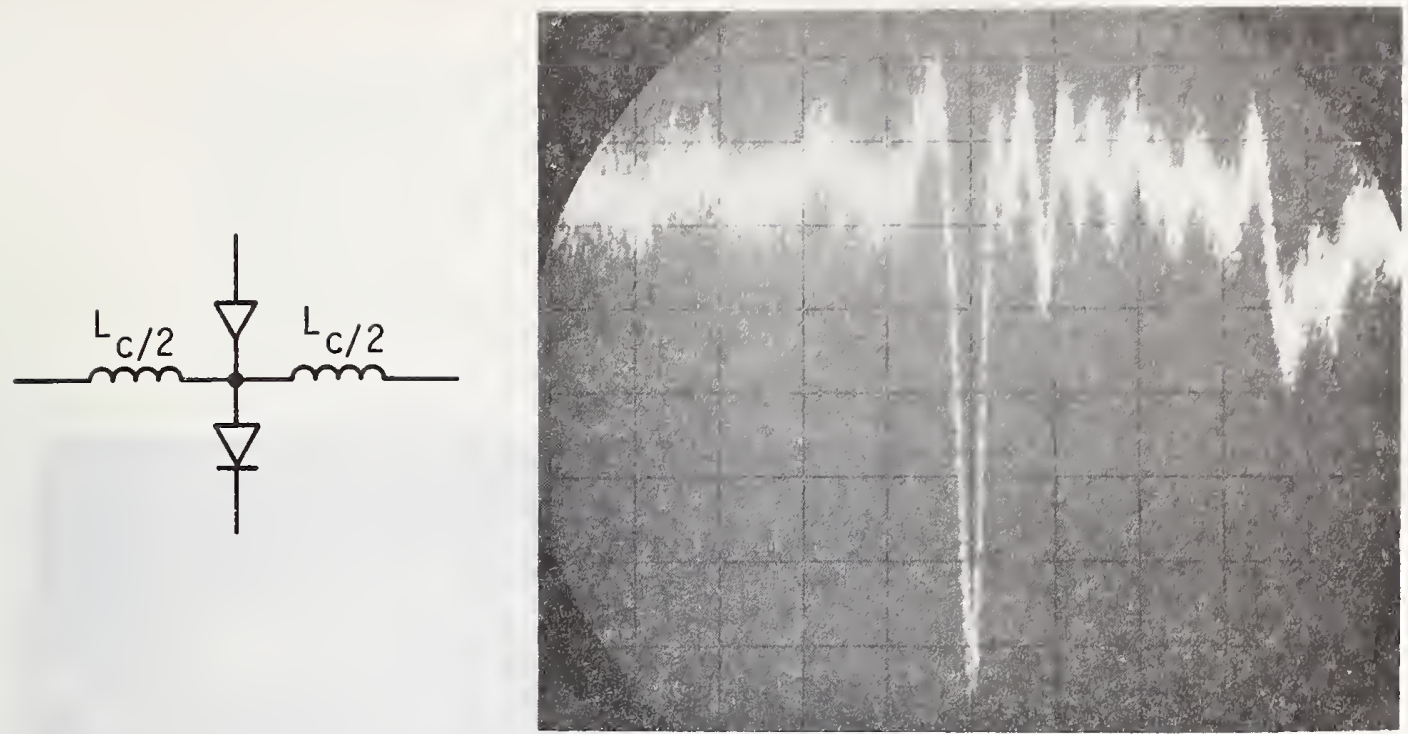

a. Two Diodes
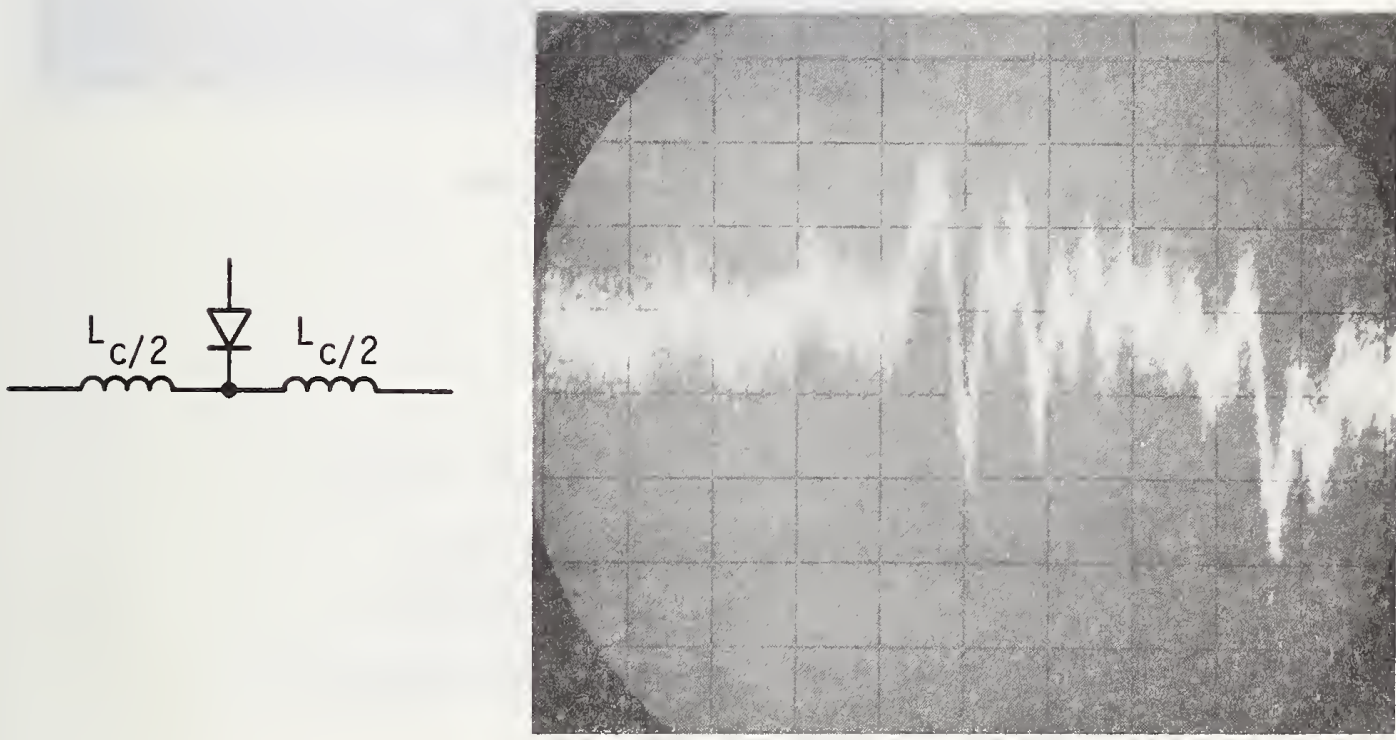

b. One Diode

Figure 6.17. To be continued on next page. 

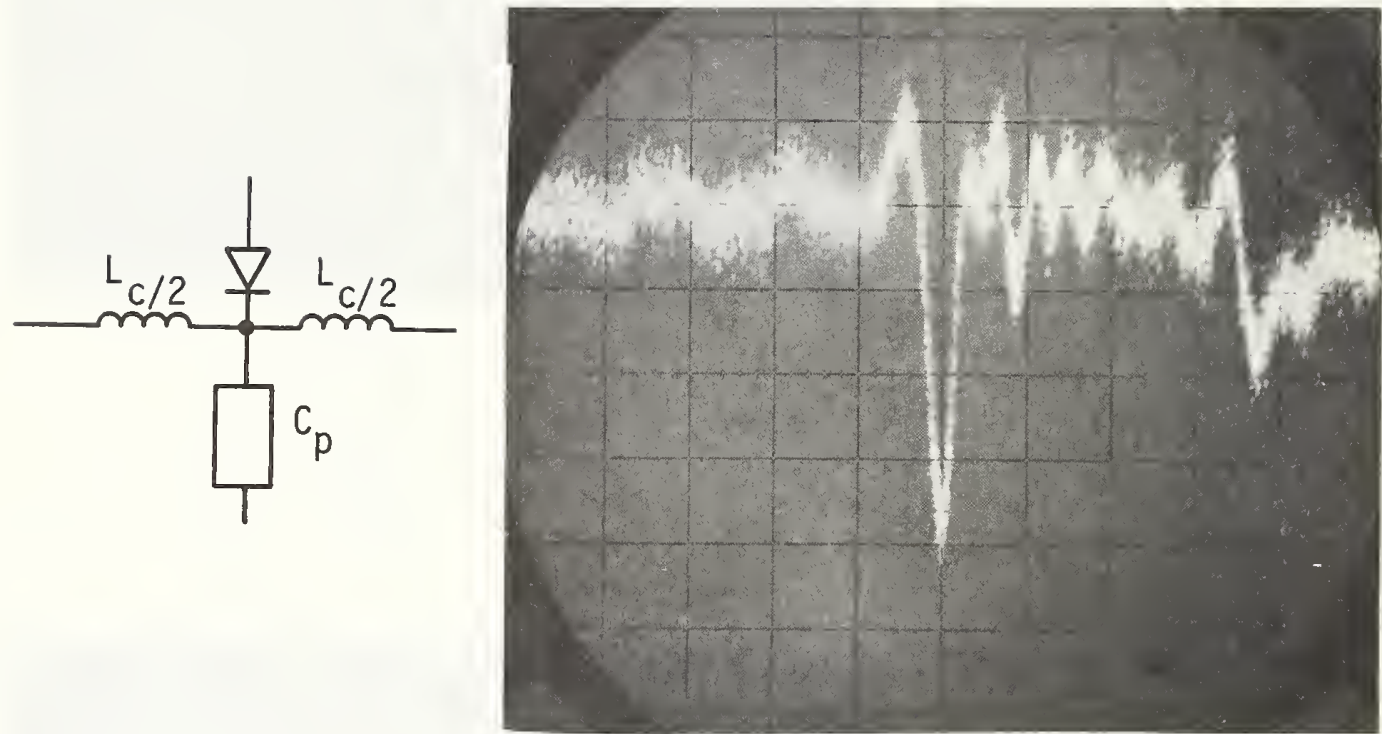

c. One Diode and an Open Diode

Figure 6.17. TDR on Diodes.

vert. $5 \mathrm{mv} / \mathrm{div}$

Horiz. 100 ps/div 


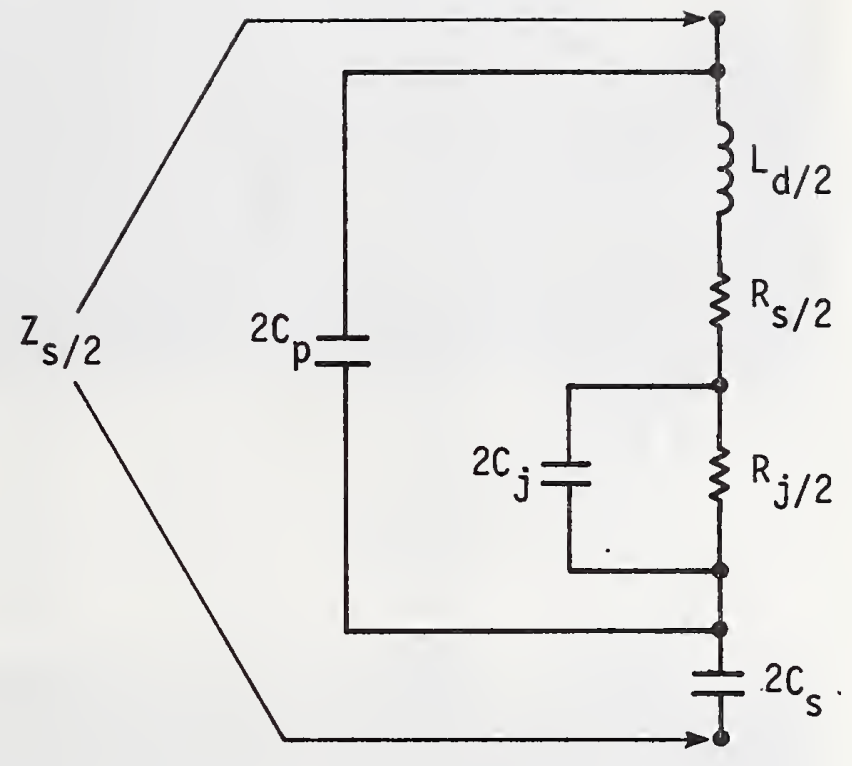

Figure 6.18. Sampling Gate Impedance $z_{s} / 2$. 


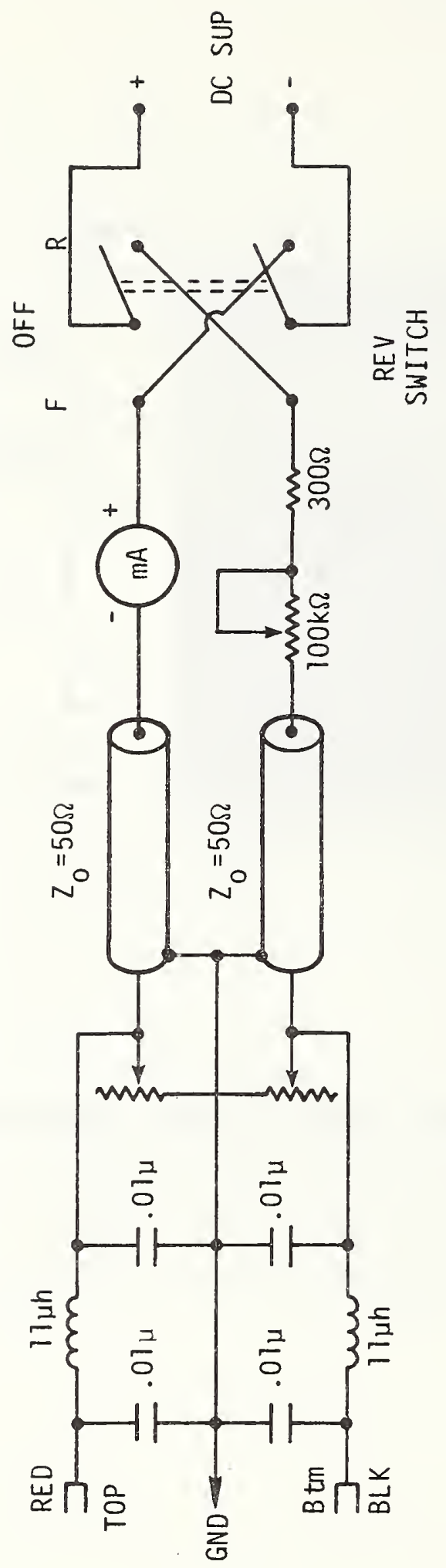

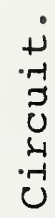

D

ڤn

वे

व) 


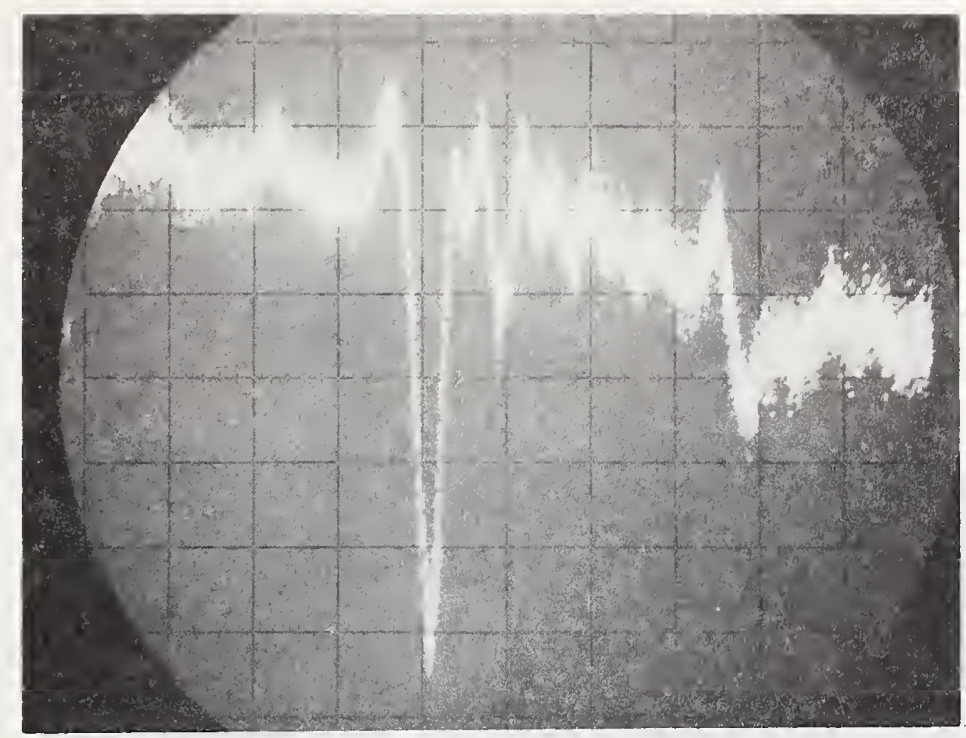

a. $v=0.0$ Volt.

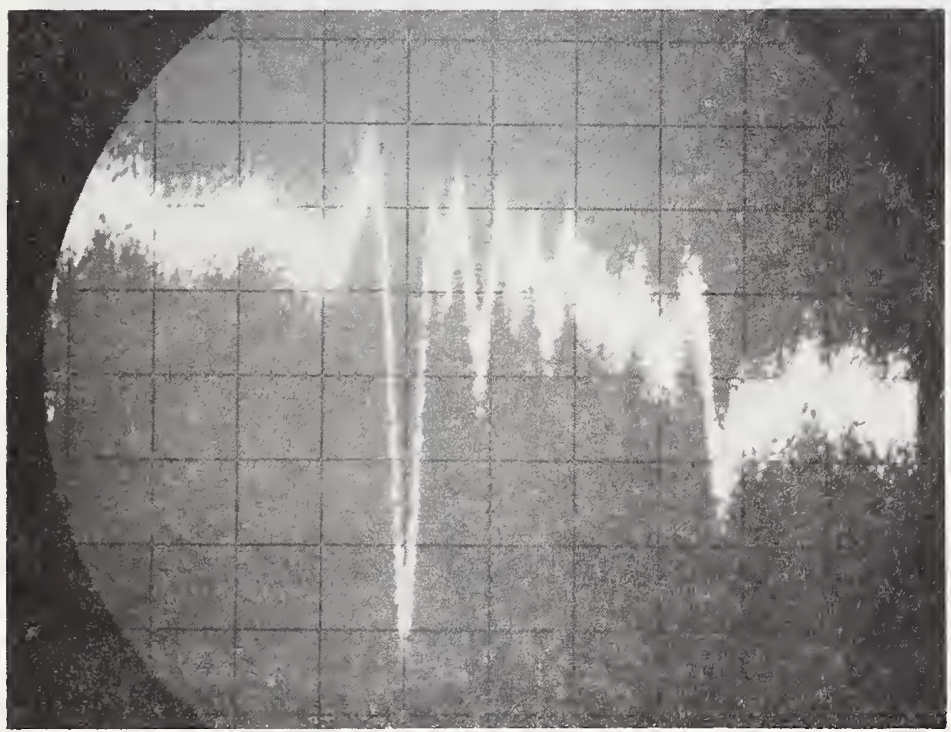

b. $v=-1.0$ volt.

Figure 6.20. To be continued on next page. 


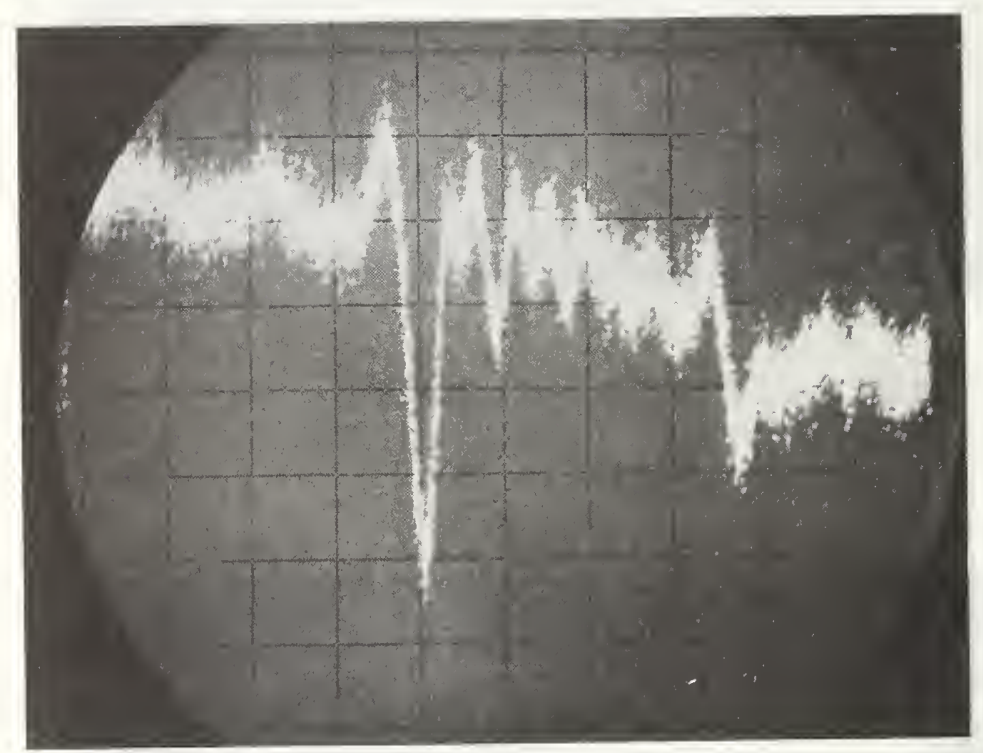

c. $\quad \mathrm{v}=-2.0$ Volt.

Figure 6.20. TDR vs. Bias.

Vert. $5 \mathrm{mv} / \mathrm{div}$

Horiz. 100 ps/div 


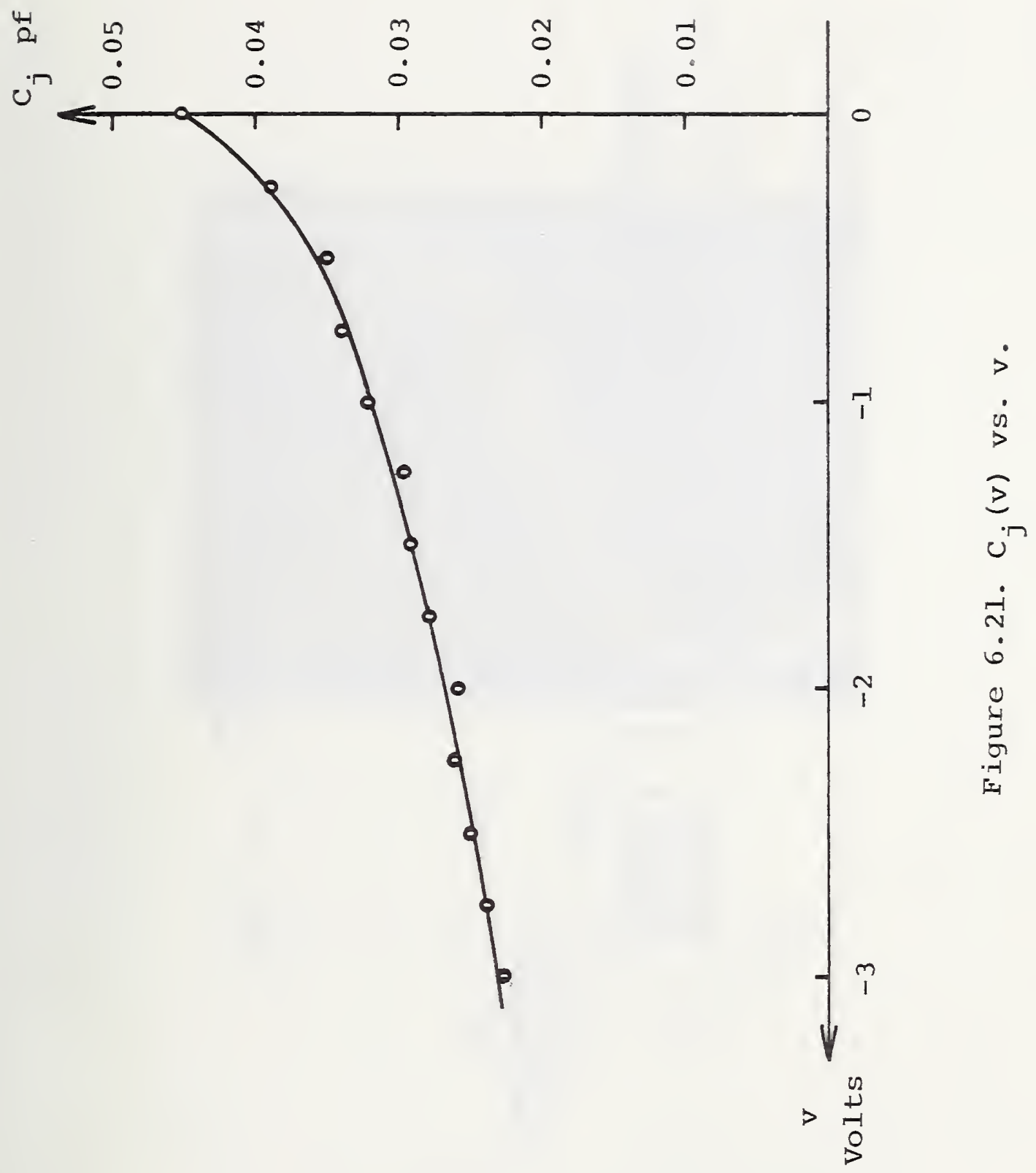




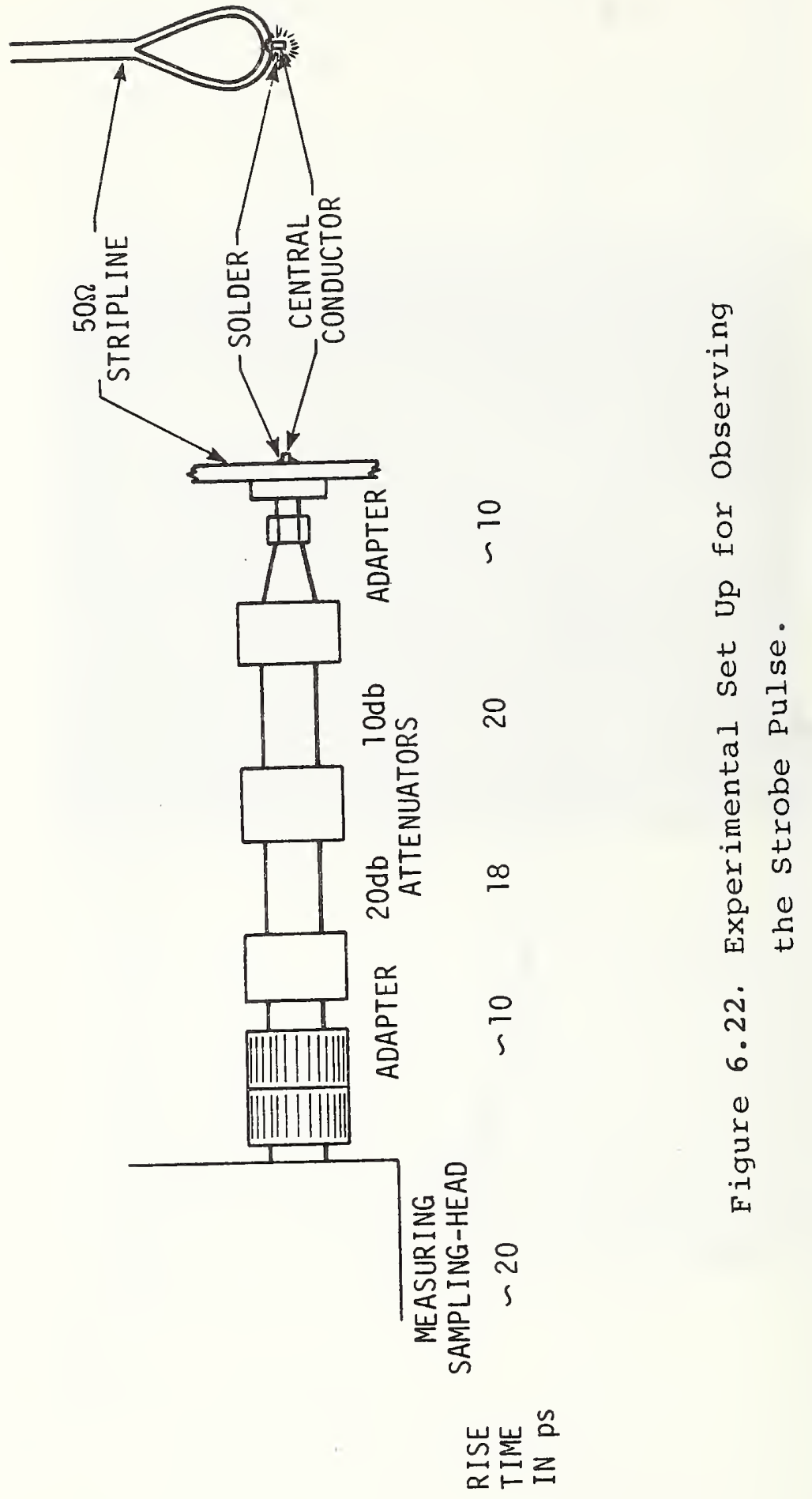




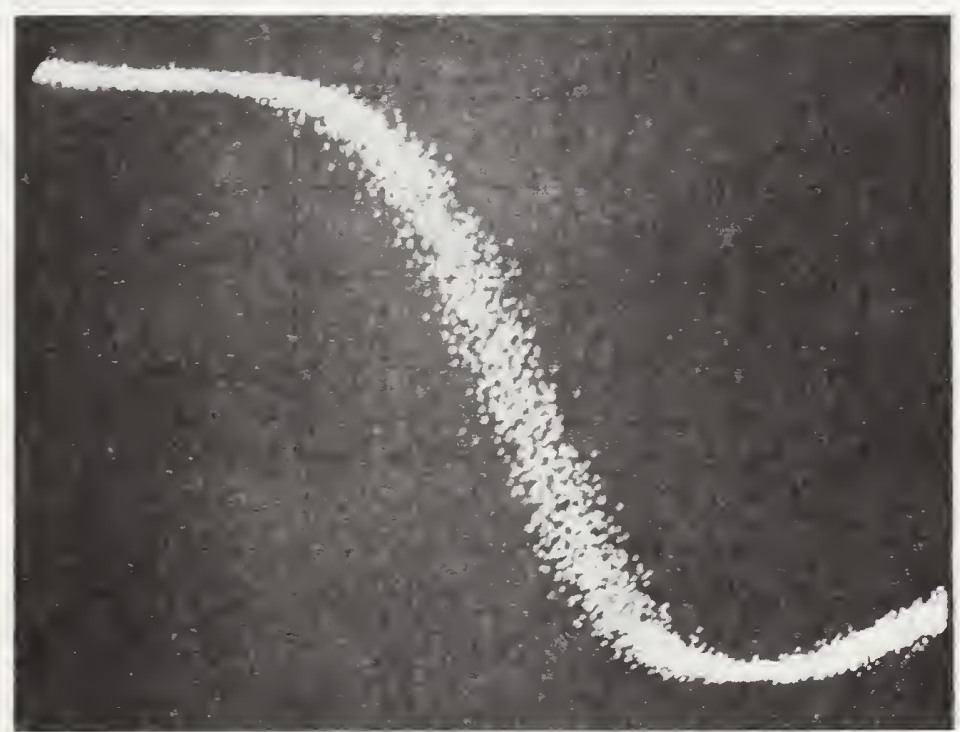

Figure 6.23, Observed Strobe Pulse. vert. $1.581 \mathrm{v} / \mathrm{div}$. Horiz. $20 \mathrm{ps} / \mathrm{cm}$. 


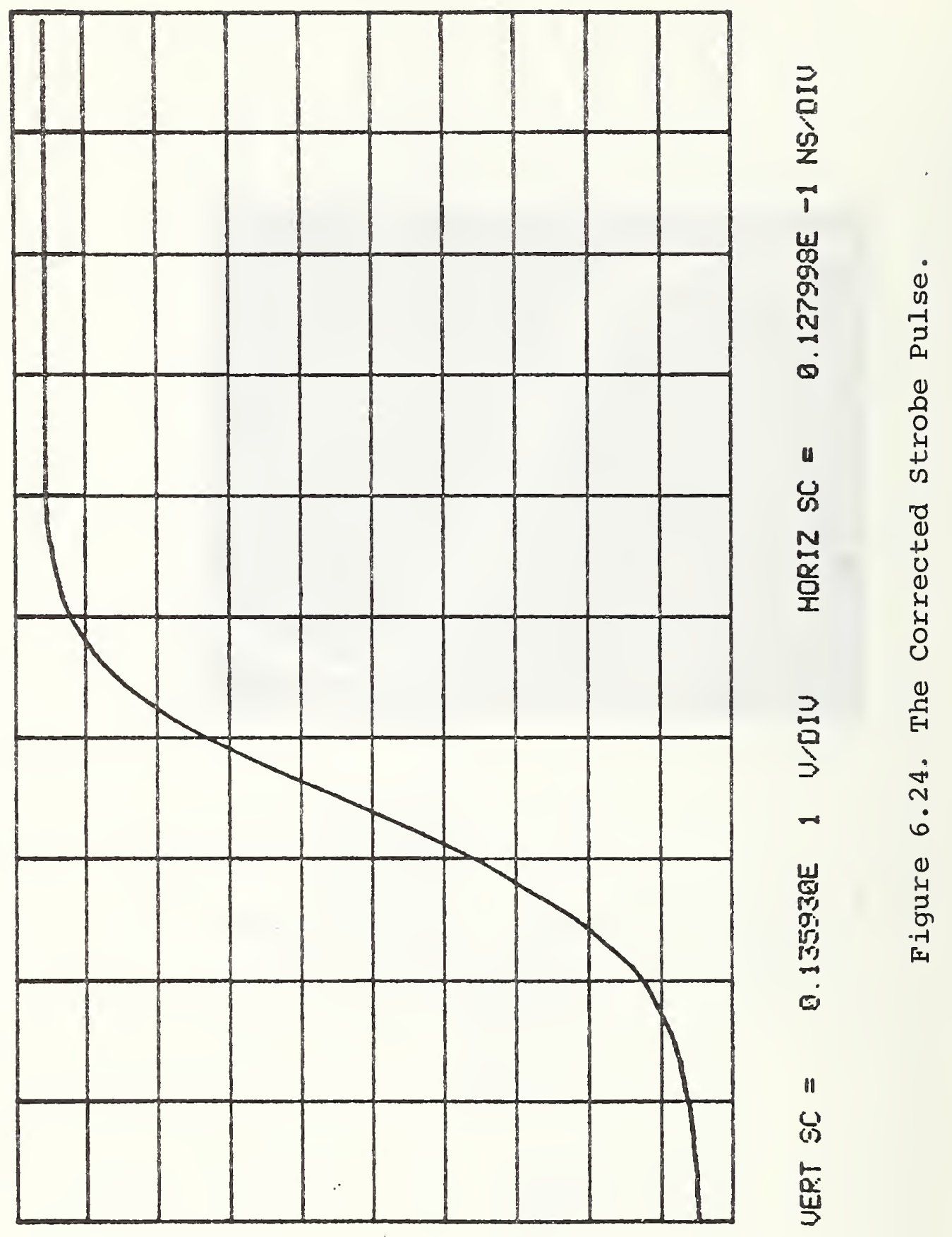




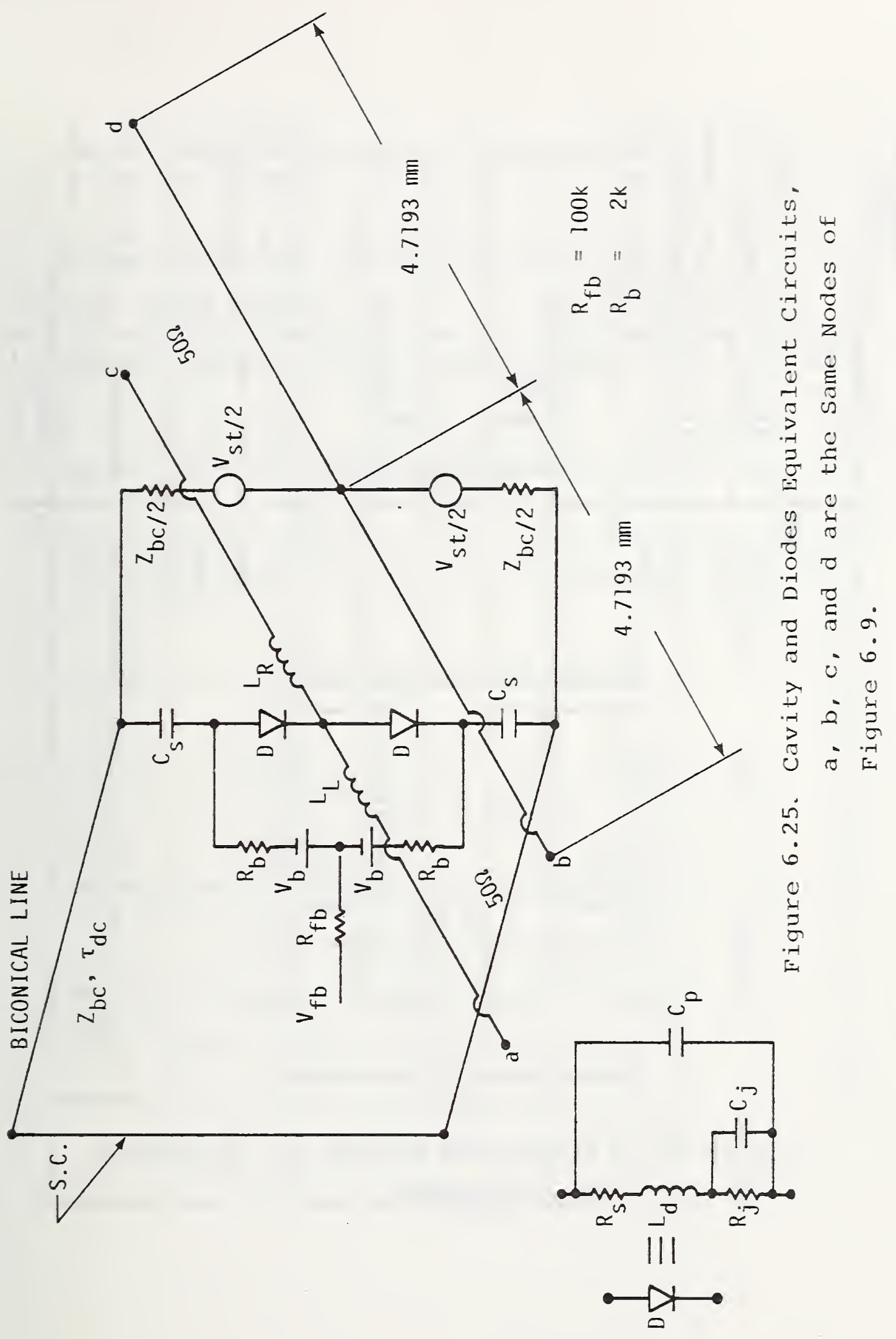




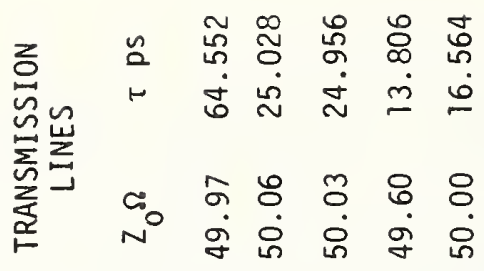

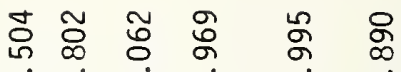

$\because \stackrel{\sim}{\sim} \dot{\sim} \infty \dot{8}$

8 g $8=$ ㅇ

㝏

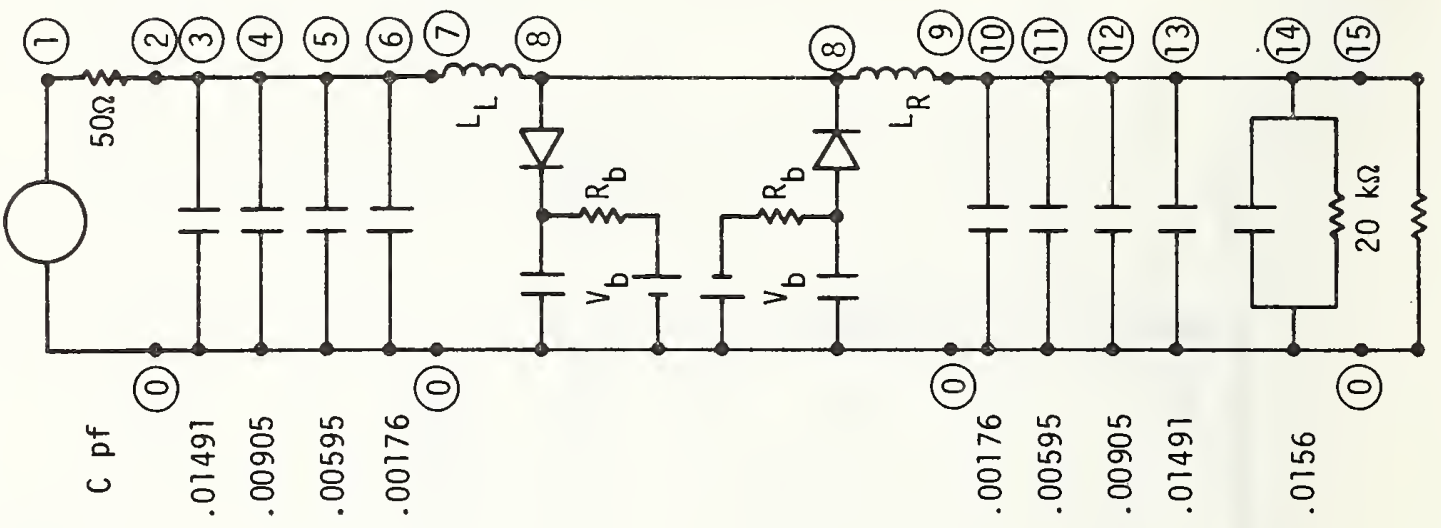

(8)

(8)

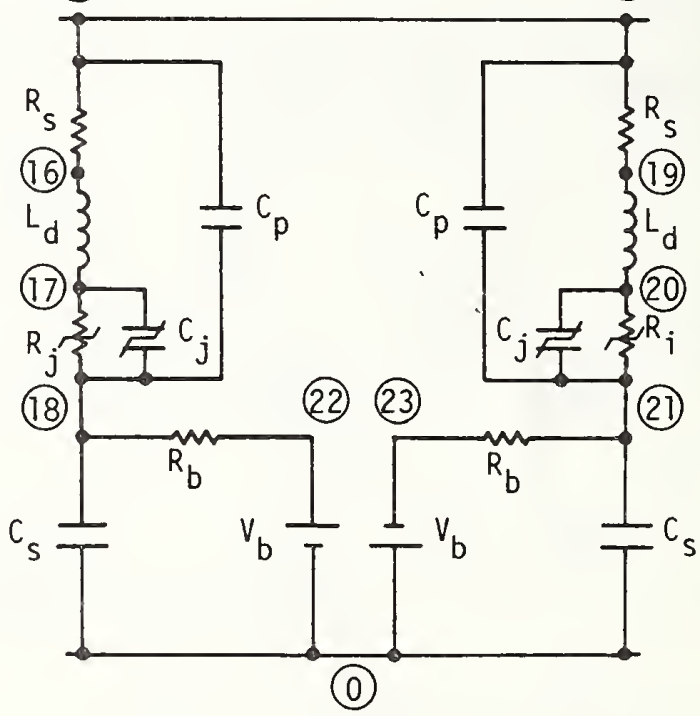

Figure 6.26. Simplified Network for Structure Step Response. 


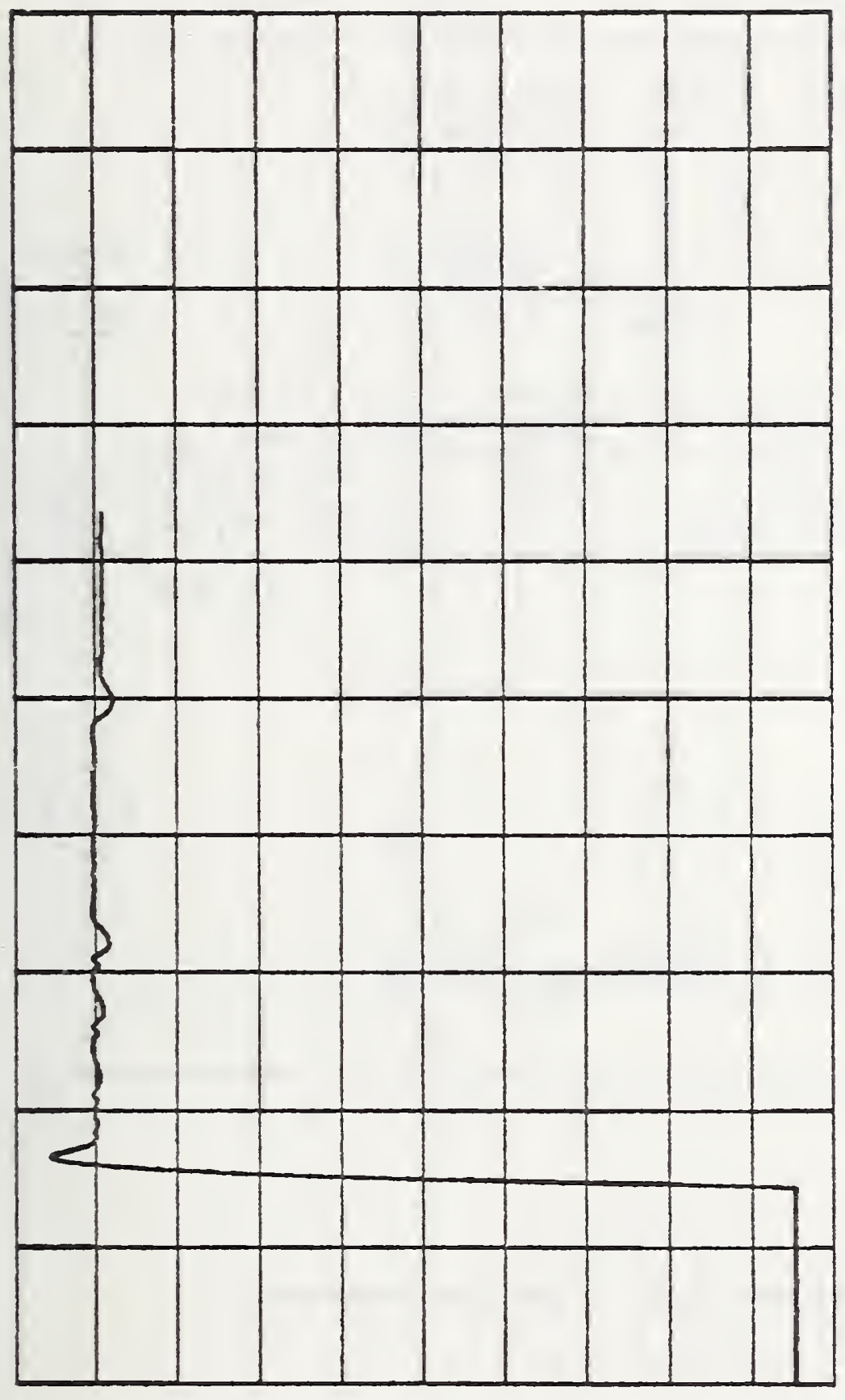

$\begin{array}{ll}\stackrel{0}{0} & \dot{0} \\ 0 & \stackrel{0}{1}\end{array}$

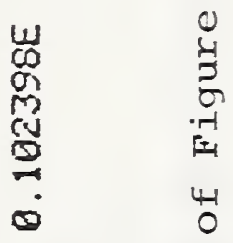

$\begin{array}{ll}11 & \geq \\ 0 & 0 \\ 0 & 0 \\ N & 0 \\ 0 & 0 \\ 0 & 0 \\ 0 & 0\end{array}$

0
4
u

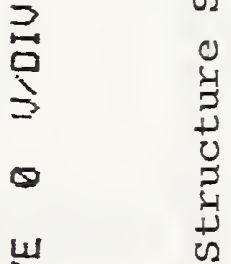

$n$
0
0
0
0

11 दू

जी

恕 


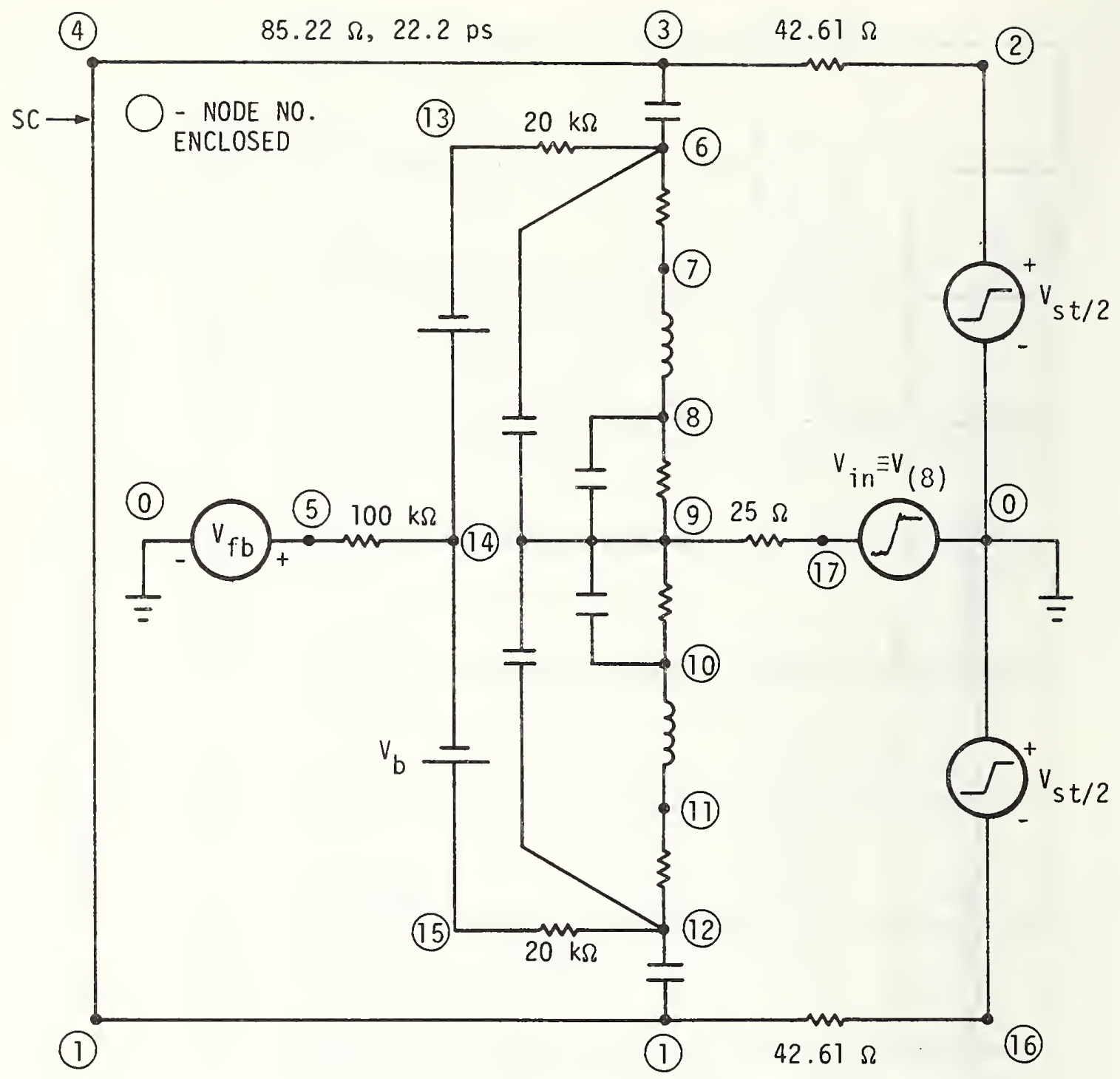

Figure 6.28. Sampling Network. 


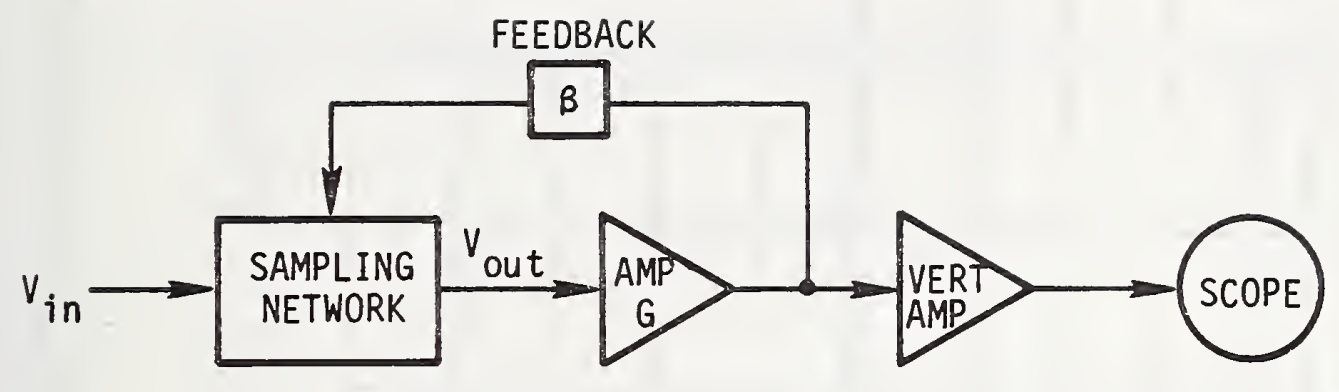

Figure 6.29. Sampling Efficiency and Feedback. 


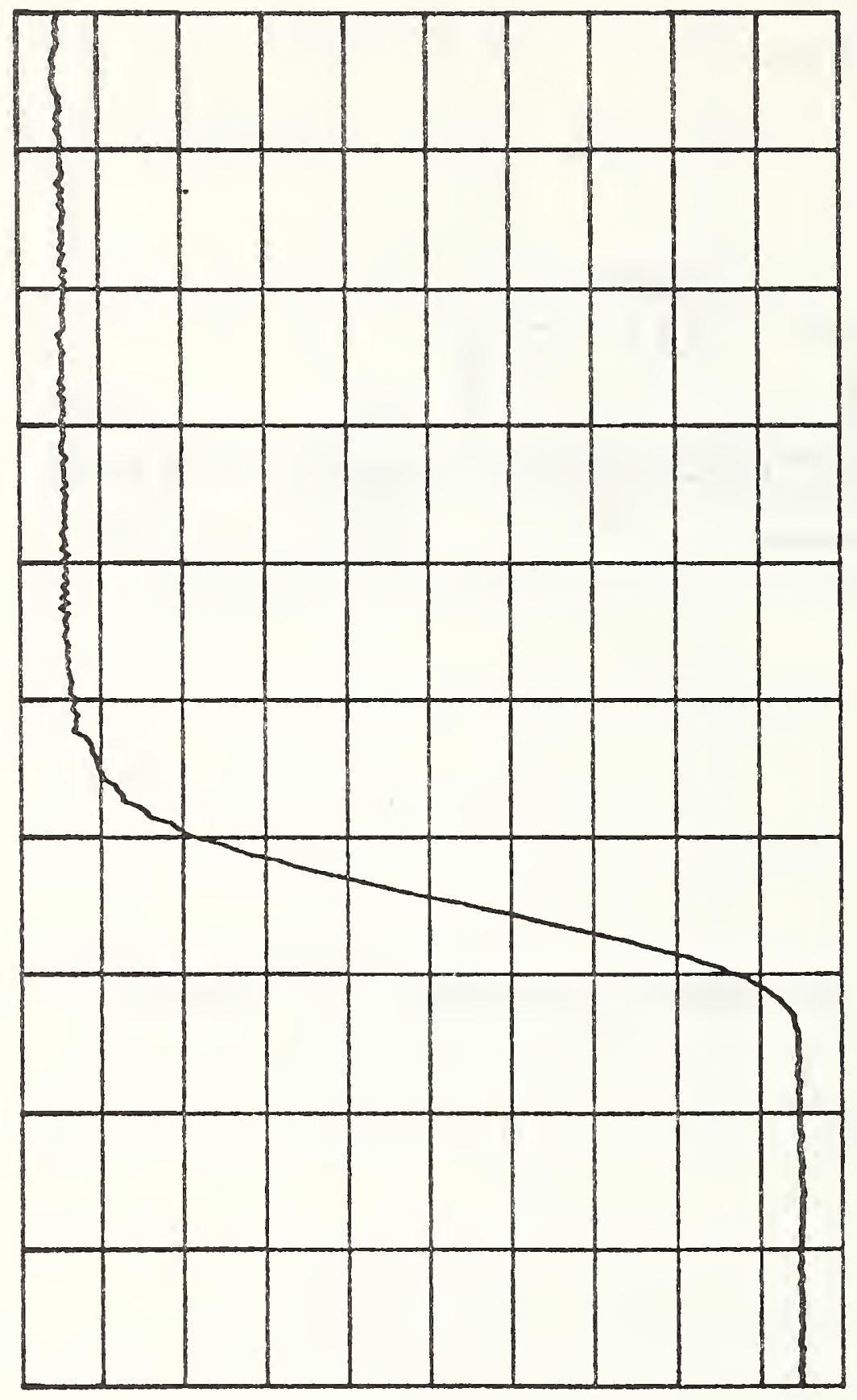

$\frac{2}{2}$

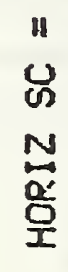

0
0
0
0
0
+1
0
0

6

분

வ.

$+$

(1)

U

0

(1)

$\infty$

0
$\square$
0

0
0
in
is
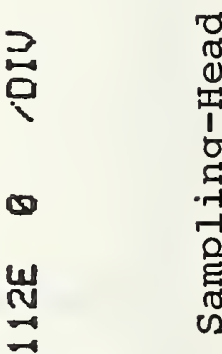

0

m

II

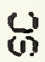

芹

紫 


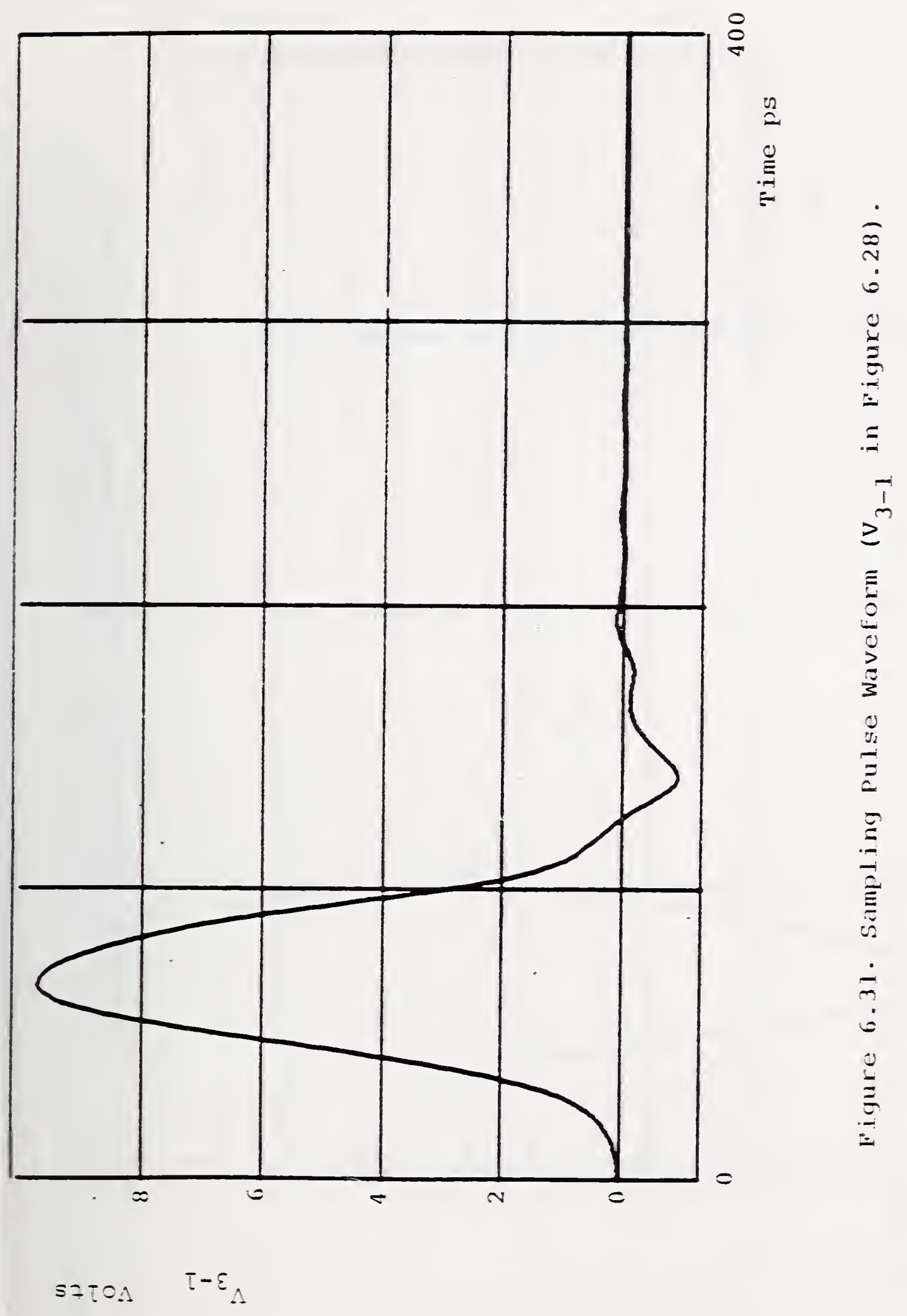




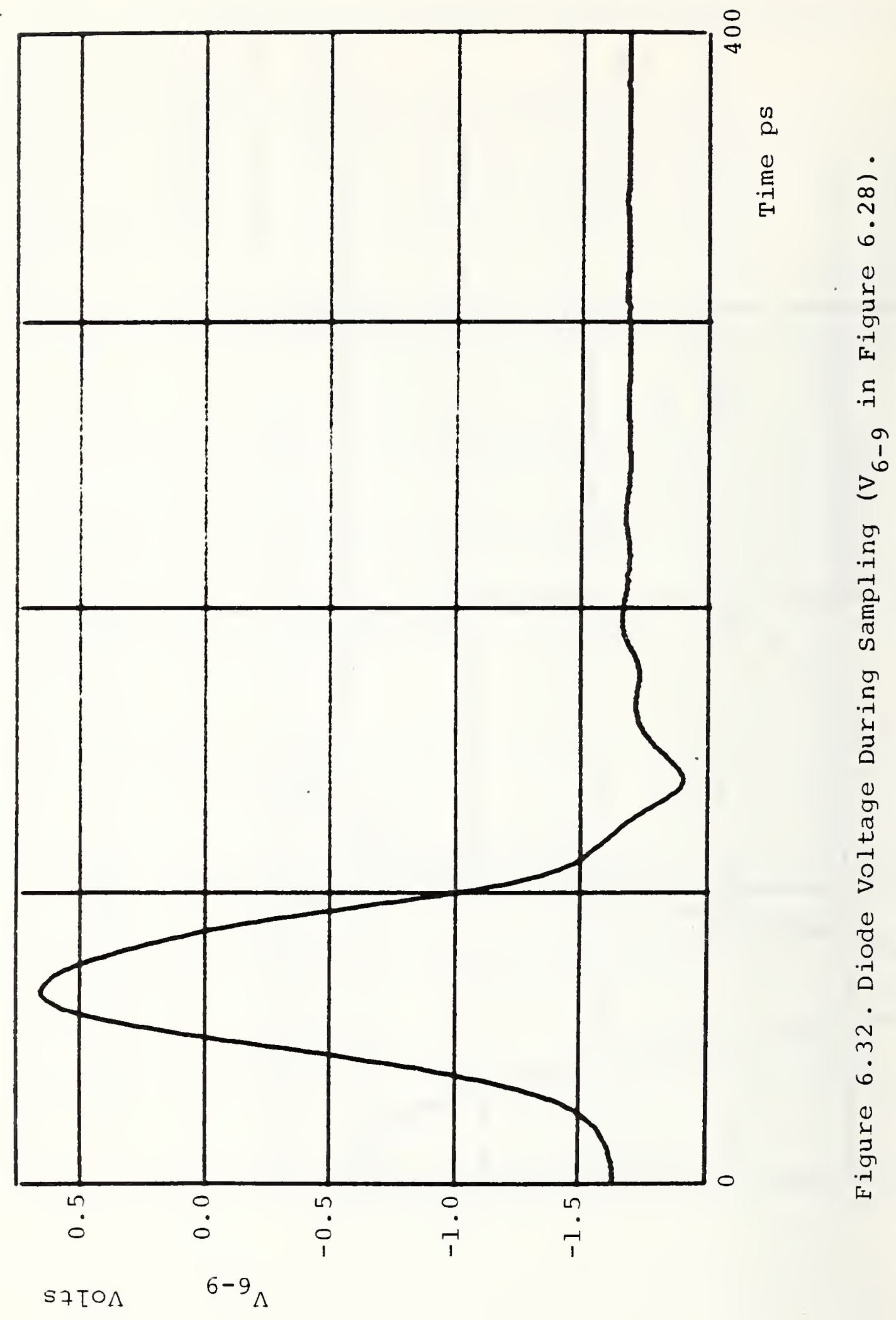




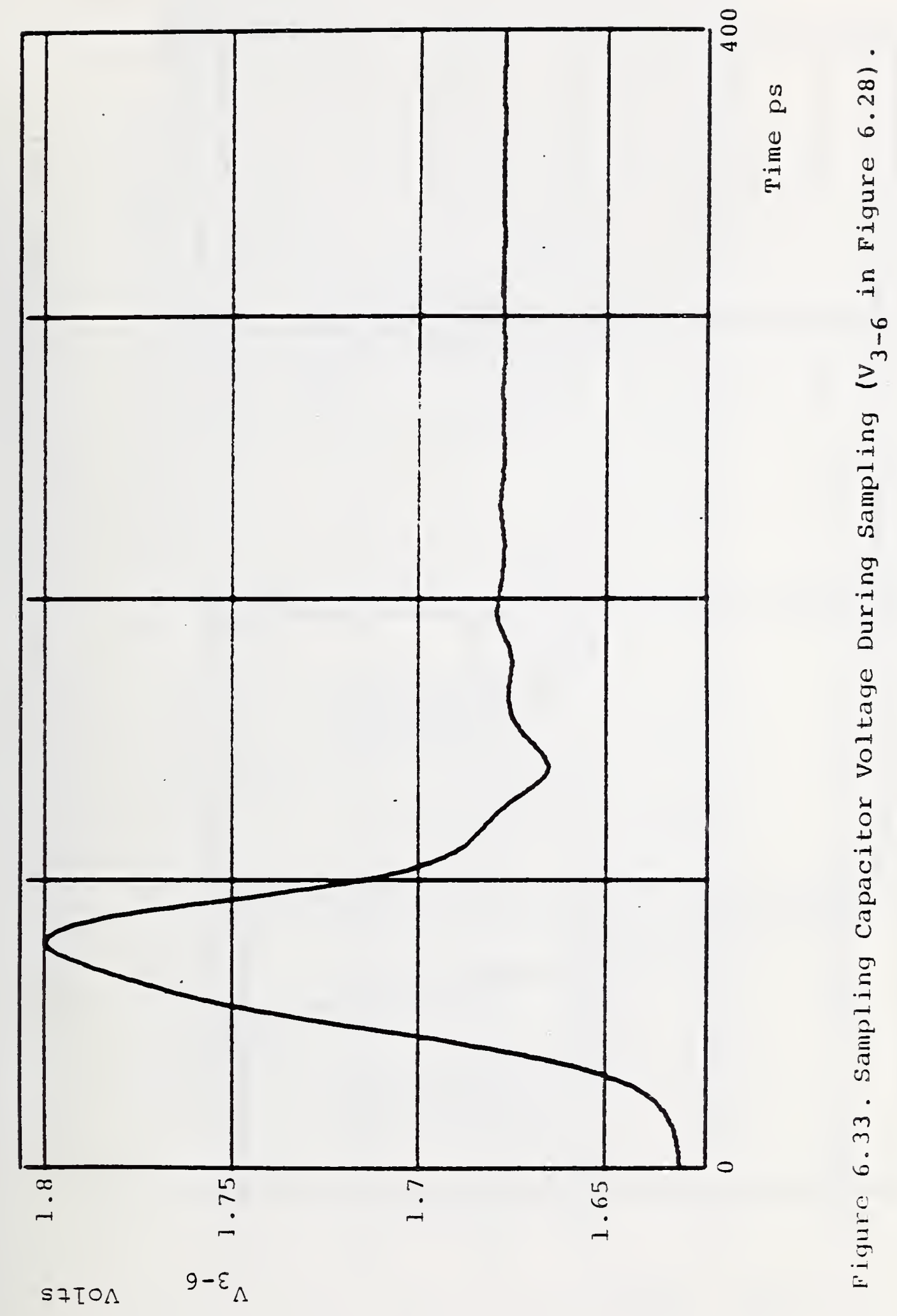




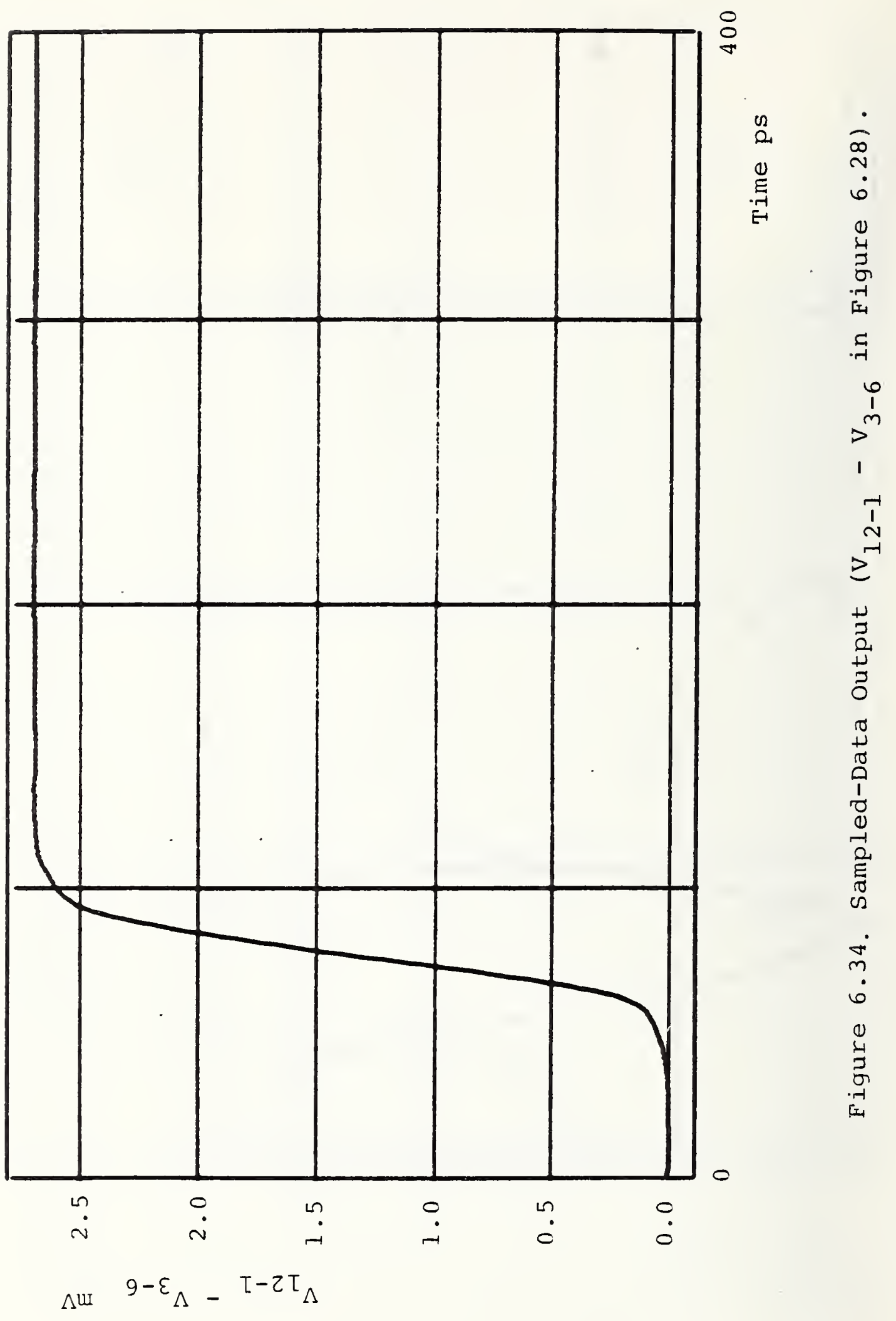



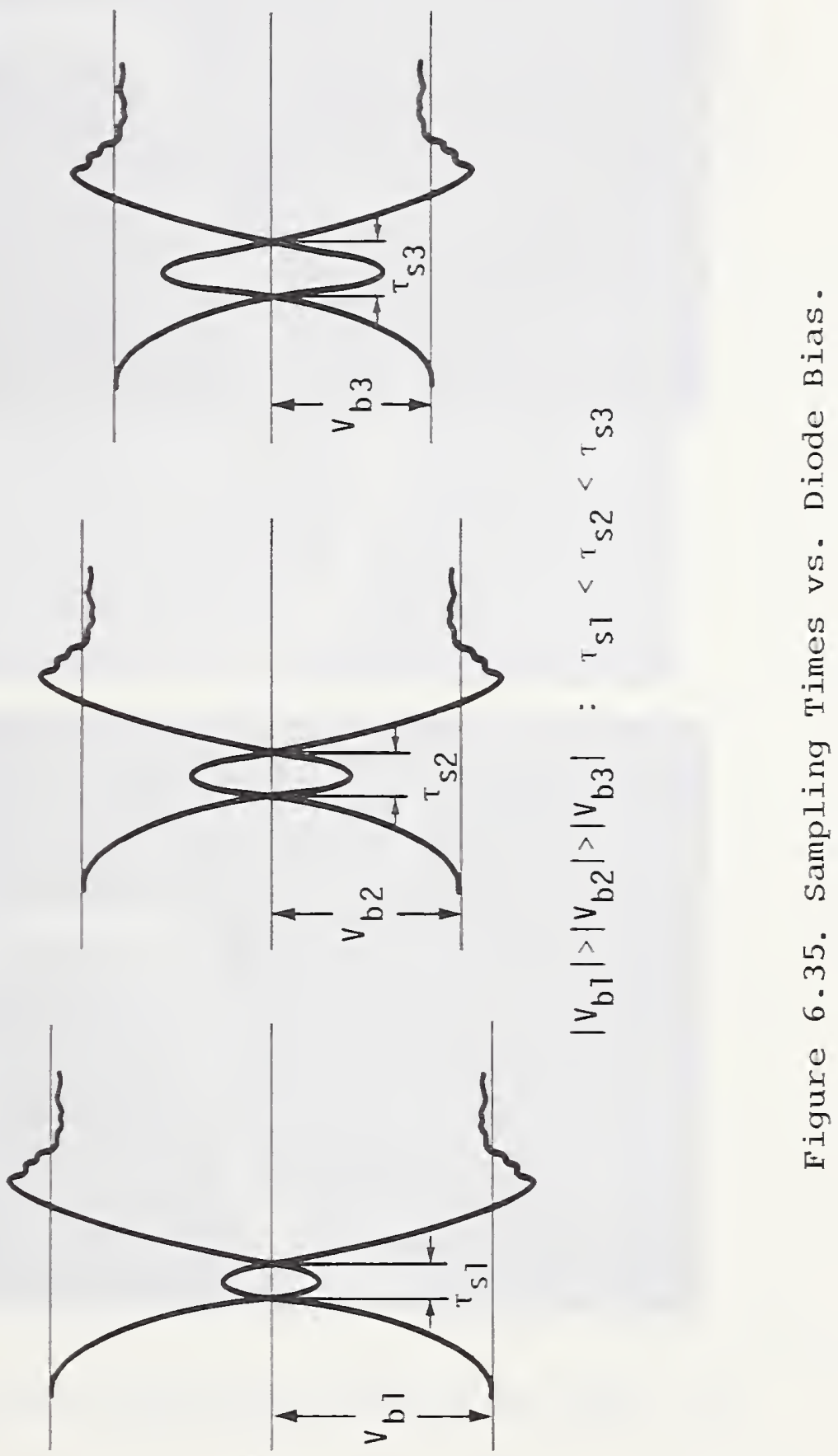


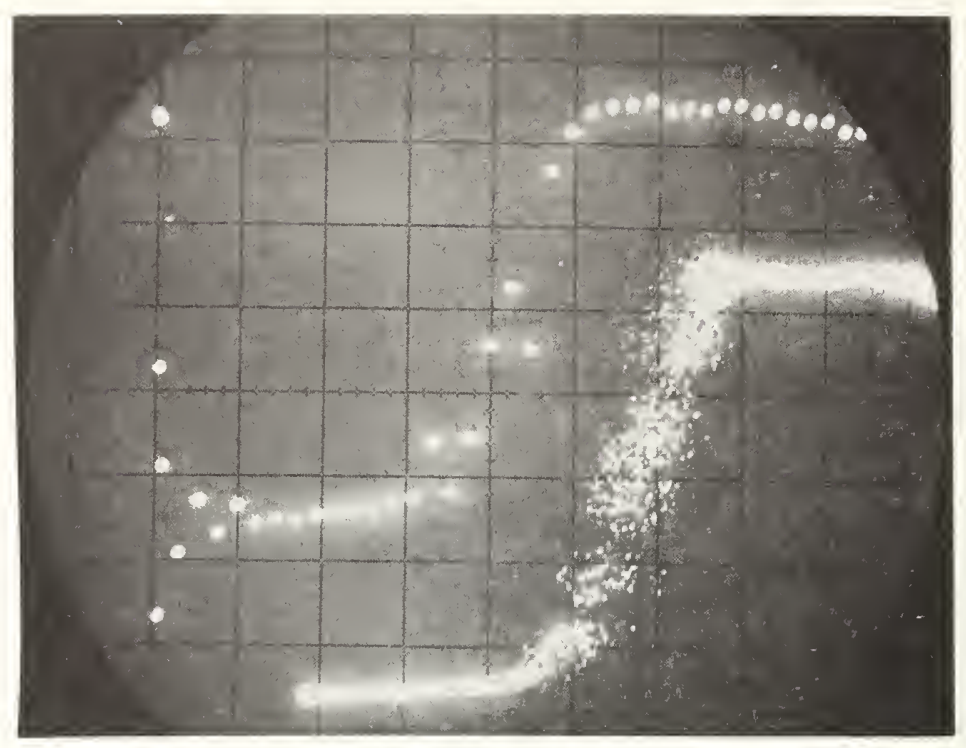

a. Low Reverse Bias ( -1.46 Volt).

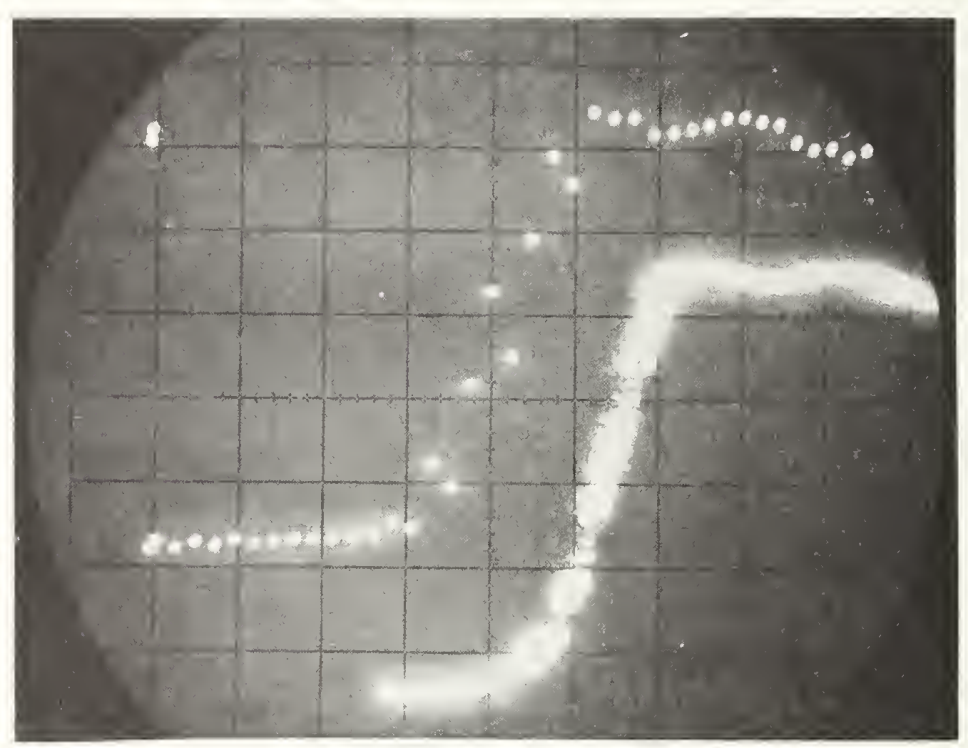

b. Bias for Optimum Sampling (-1.63 Volt).

Figure 6.36 . To be continued on next page. 


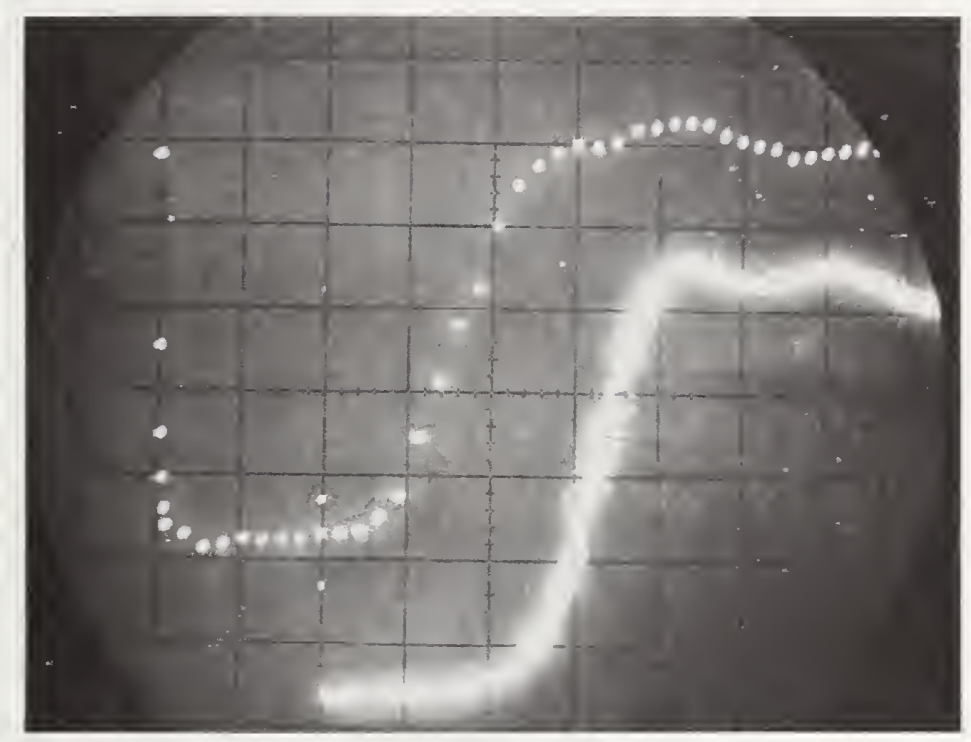

c. High Reverse Bias ( -1.76 Volt).

Figure 6.36. Effect of Diodes' Reverse Bias on Sampling. vert. $50 \mathrm{mv} / \mathrm{div}$. Horiz. 20 ps/div. 


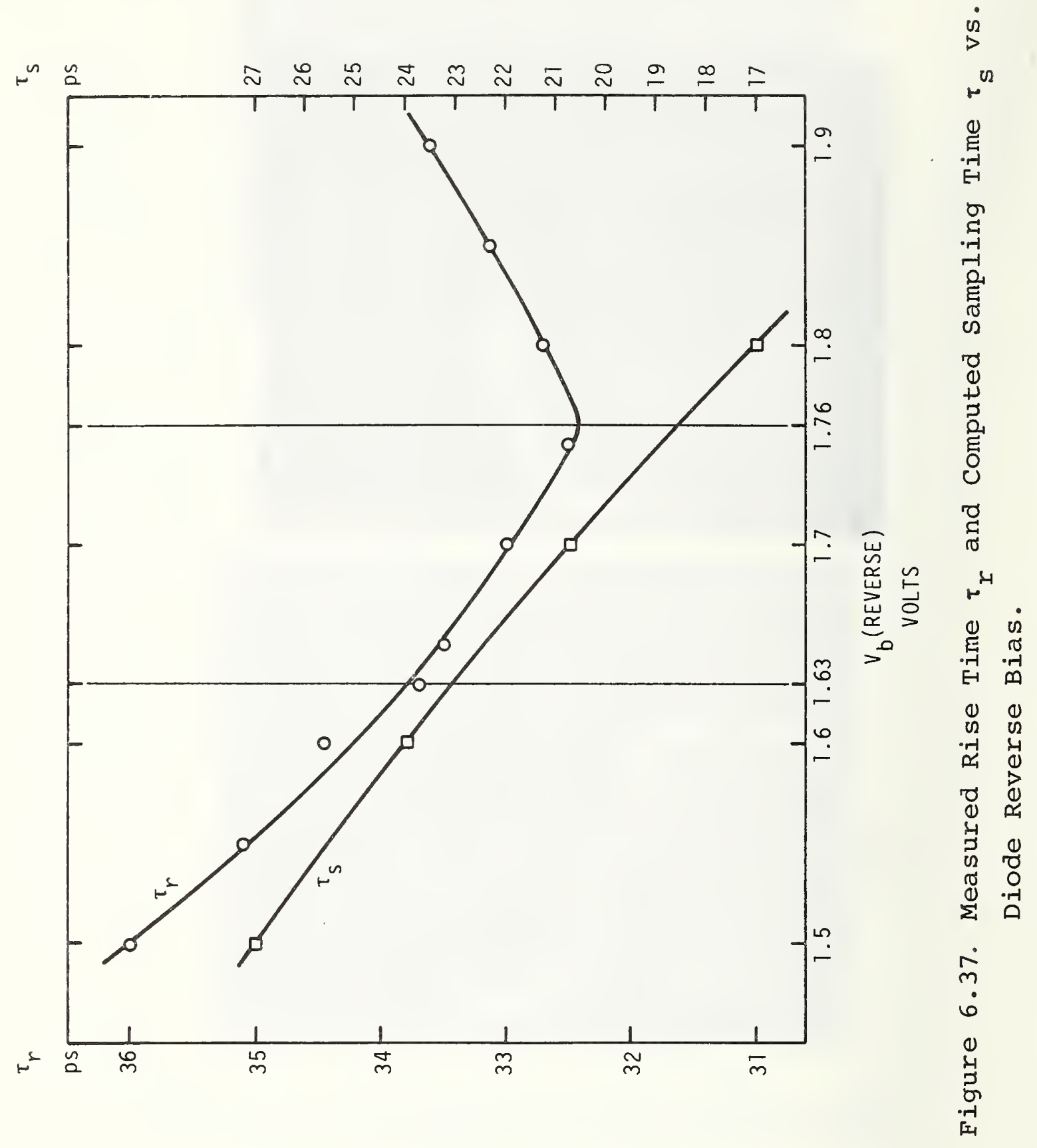




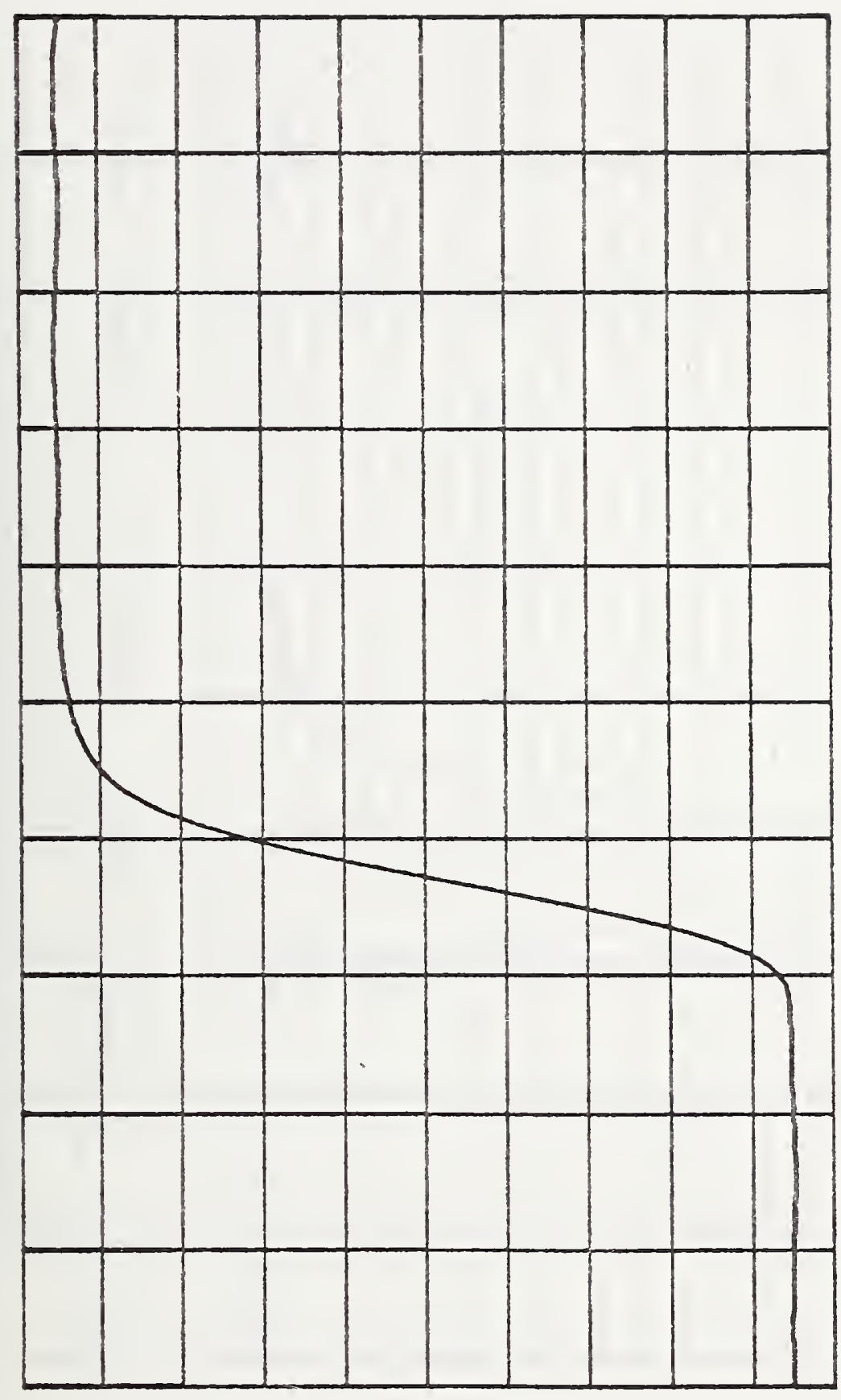

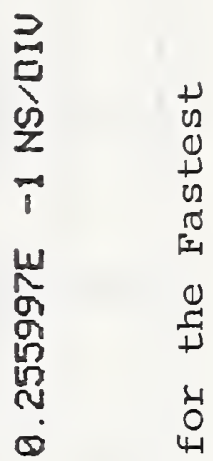

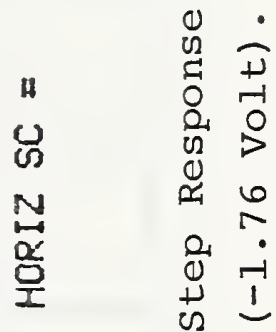

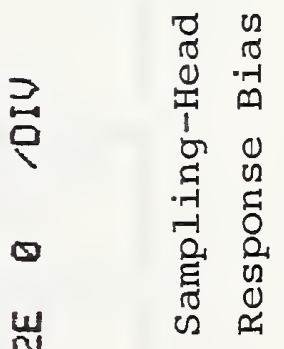

0
$=$
$=$

II

(i)

m

察 


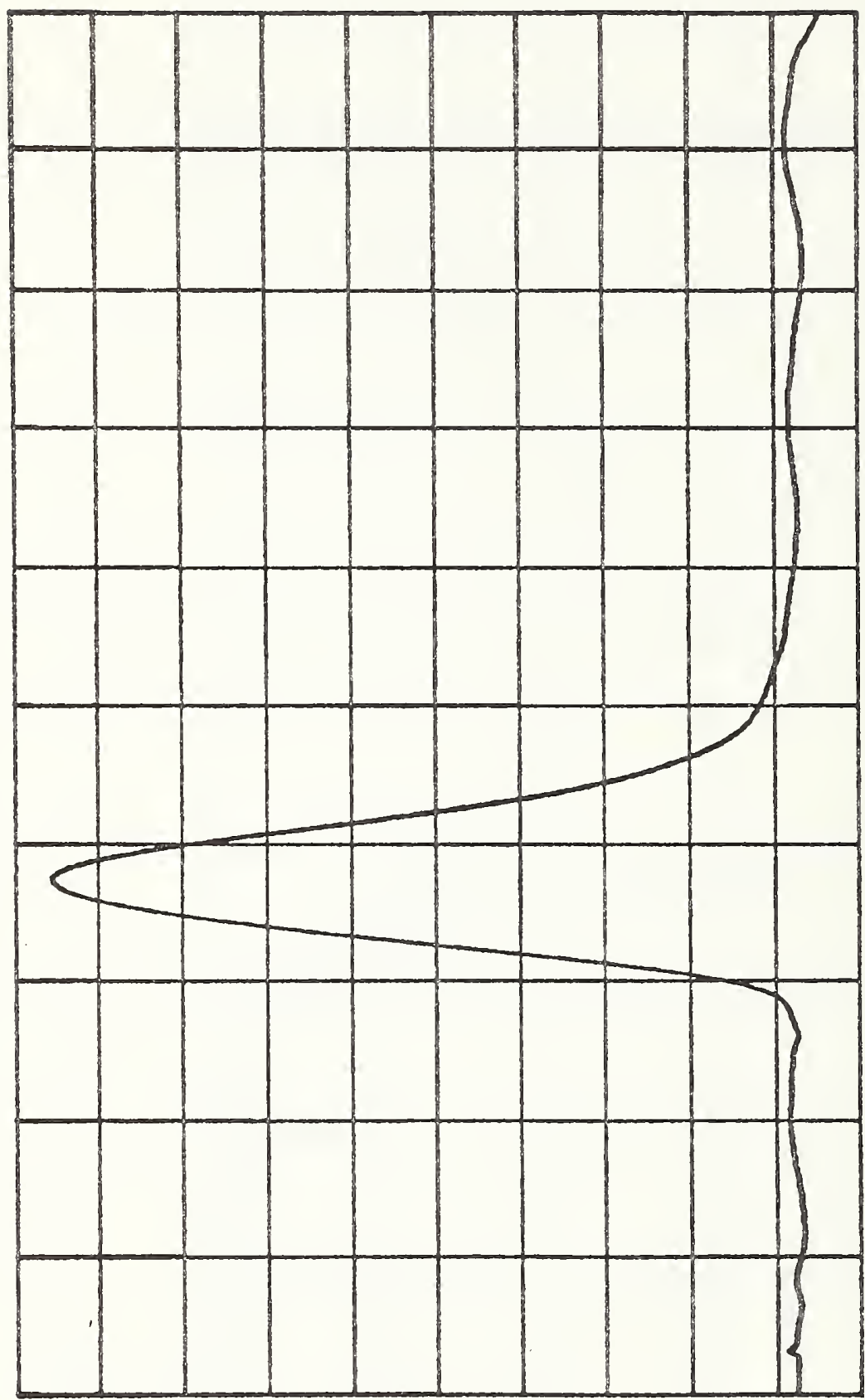

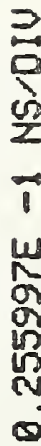

$+$

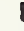

$\frac{0}{0}$

$\pm$

踏

(1)

告

(

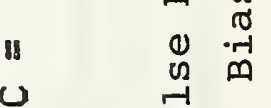

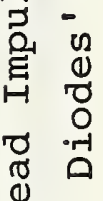

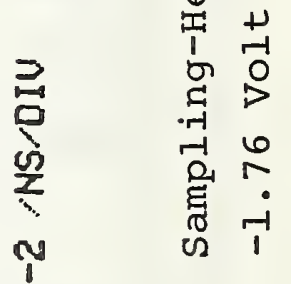

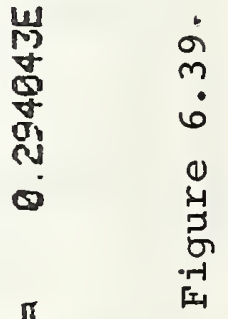

$\pi$

$\frac{(1)}{61}$

㟧 


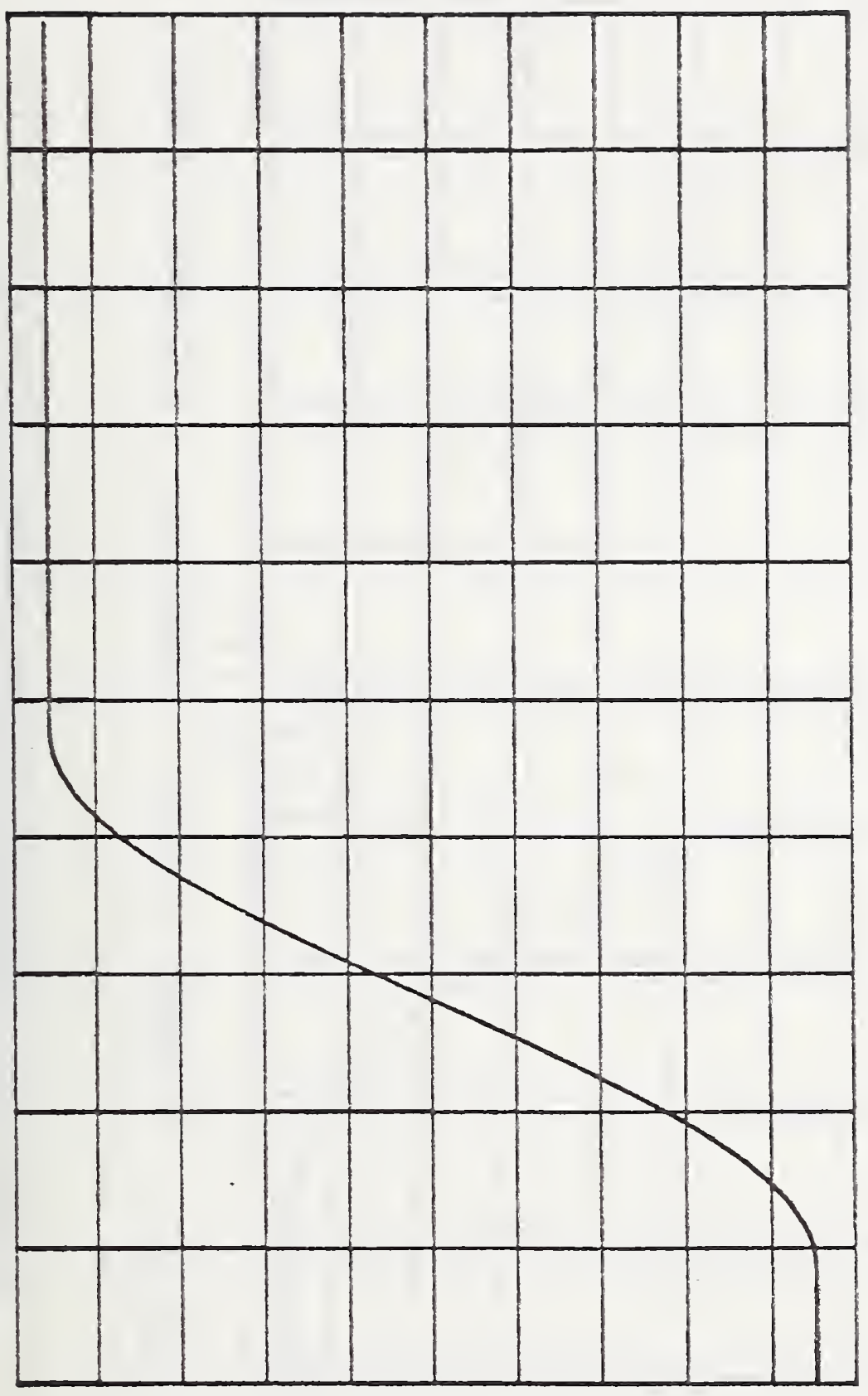

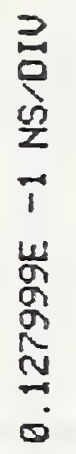

$\pm$

II

0

$\frac{N}{2}$

$x^{\pi}$

तु
हू
थ

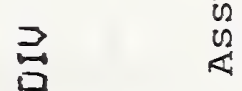

$\frac{1}{2}$

c)

$\dot{0}$
0

$w$
$N$
$N$

峁

है

4
$\frac{1}{10}$
$\stackrel{5}{2}$ 


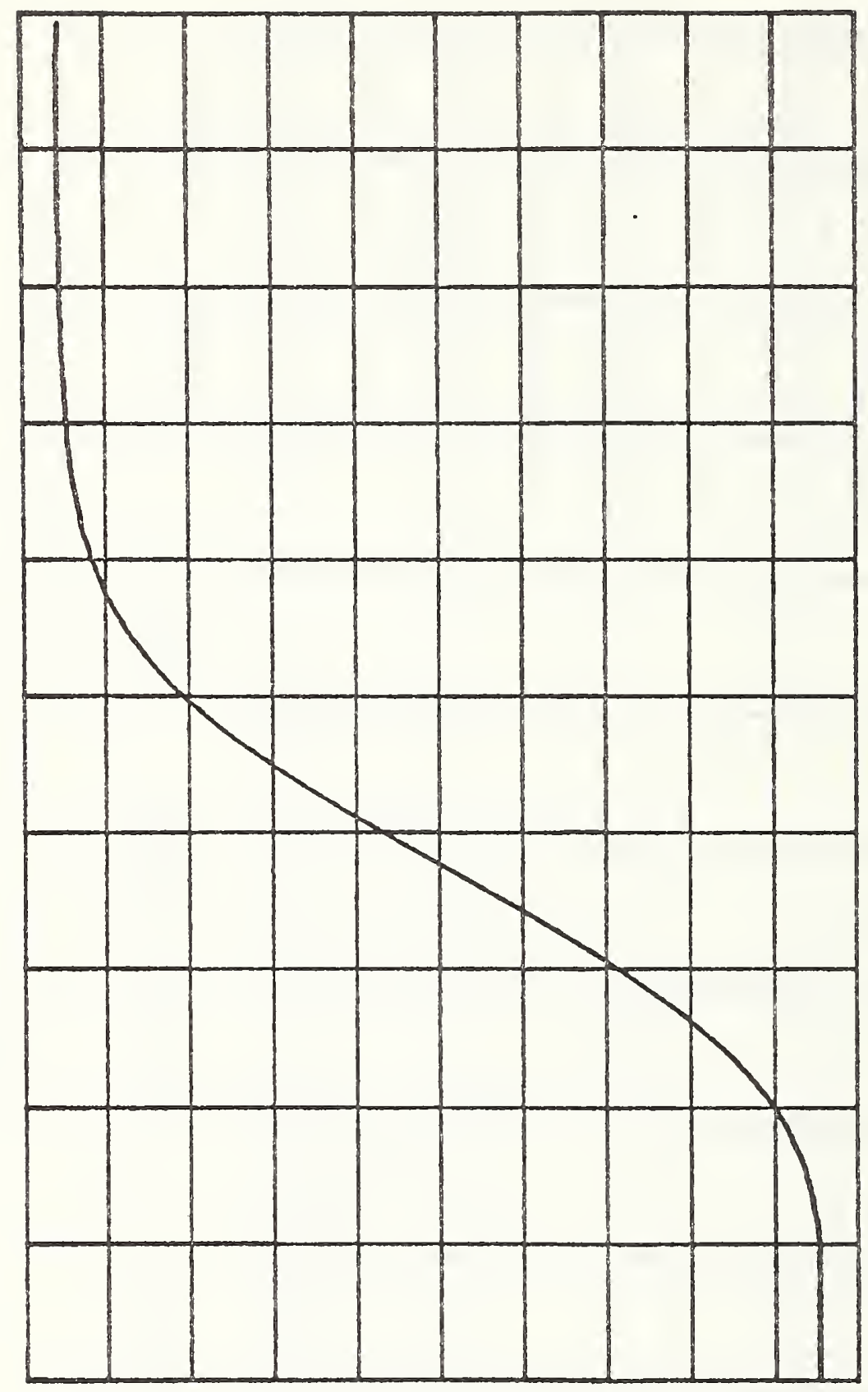

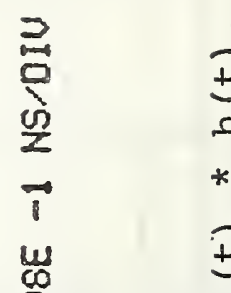

\% $x^{\sigma}$

N

II

$\underbrace{}_{\lambda}$

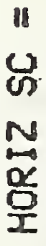

न1

$\stackrel{1}{4}$

*

$\pm$

$\infty$

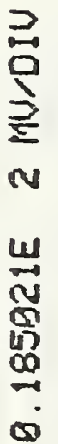

$1+1$
3
0
4
3
0
0
0
0
0
0
0
0
0

$\stackrel{-}{-}$

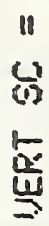

崩 


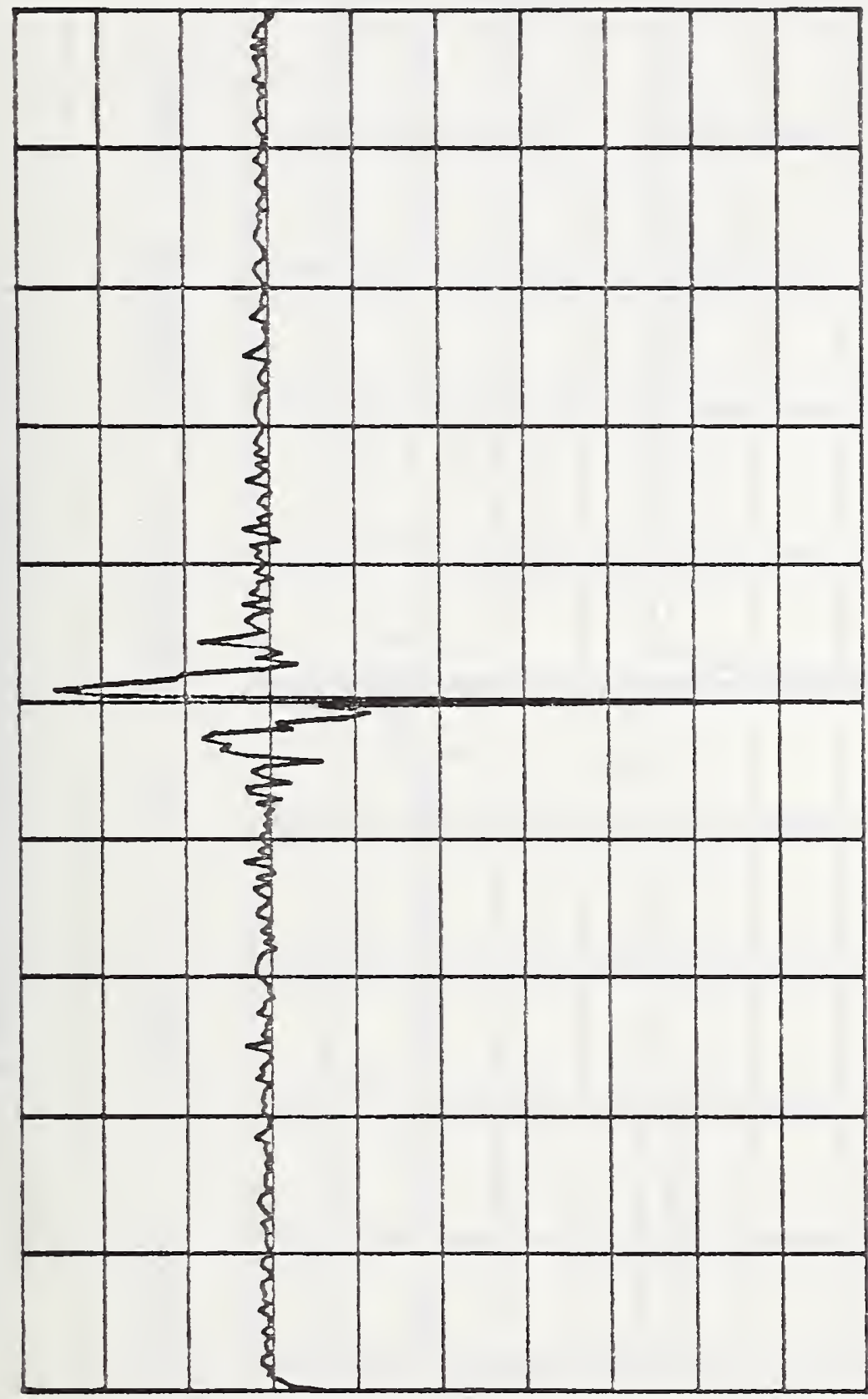

总

$\frac{1}{2}$

4

$110 \overparen{D}$

논

हี

号

E

p)

$\stackrel{\dot{\sim}}{\dot{*}}$

$\underset{0}{\infty}$

II

$\frac{1}{6 !}$

$\stackrel{5}{\underline{w}}$ 


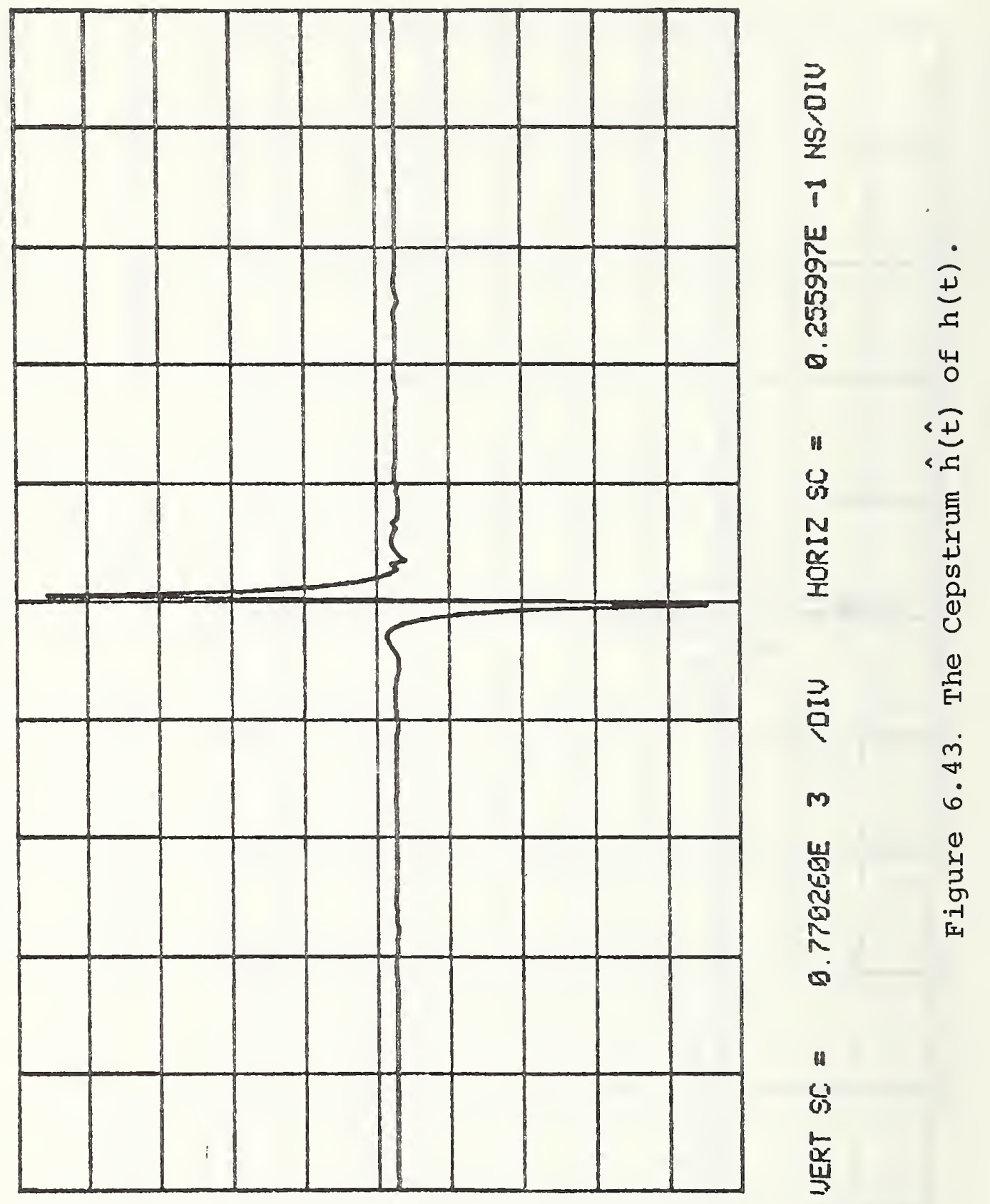




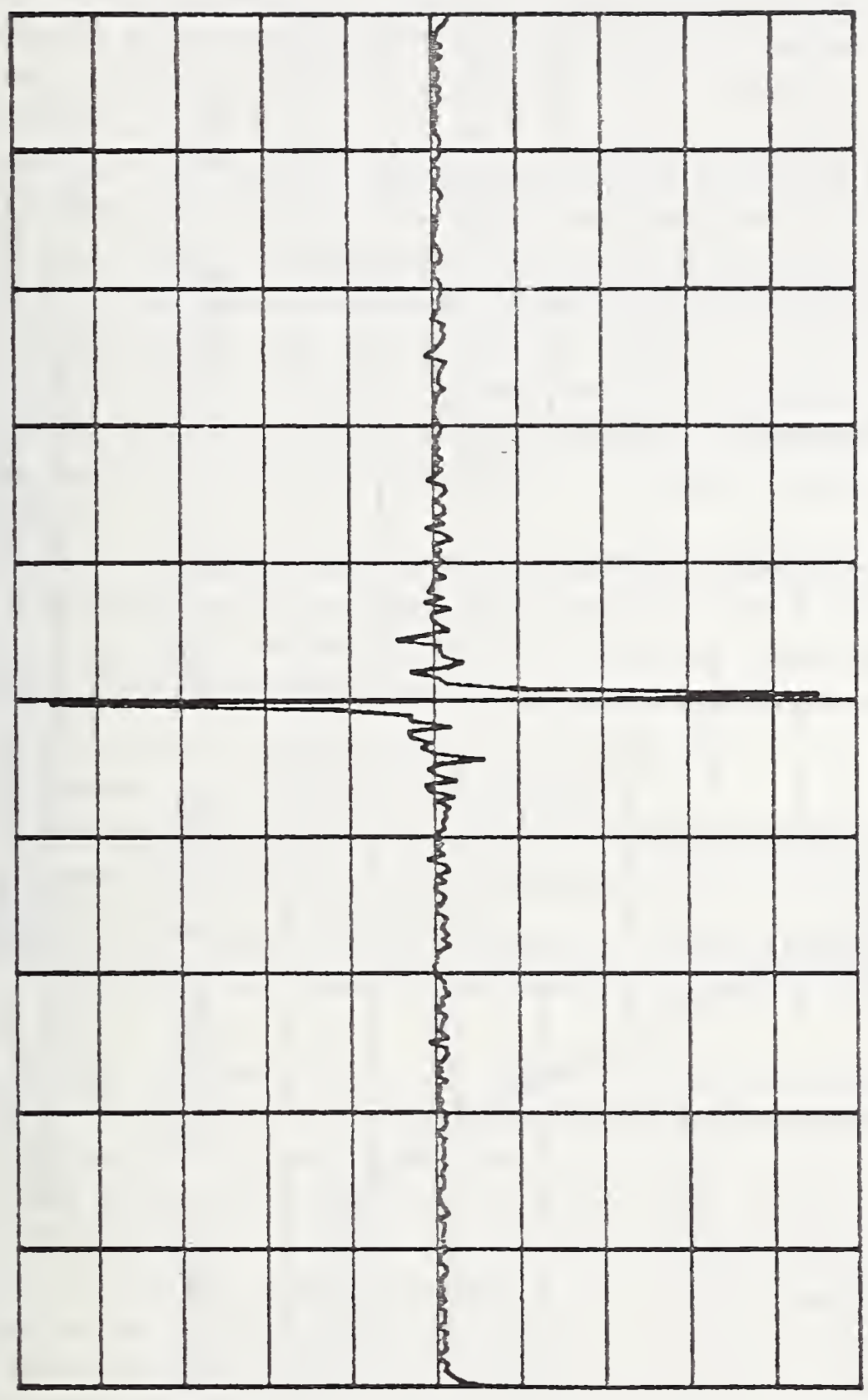

2
$\frac{5}{2}$
$\frac{5}{1}$
$\frac{1}{0}$
0
0
0

$\frac{0}{0}$

$\therefore$

I

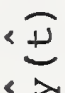

in $<$

थै

ब 2

$x^{\circ}$

$\frac{2}{2}$

0
0
0
0
0
$4-1$
44
01

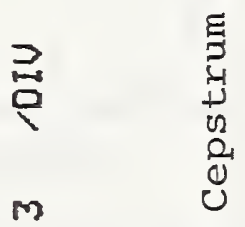

W

$\frac{7}{4}$

$\underset{0}{\dot{0}}$

$\frac{4}{0}$

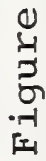




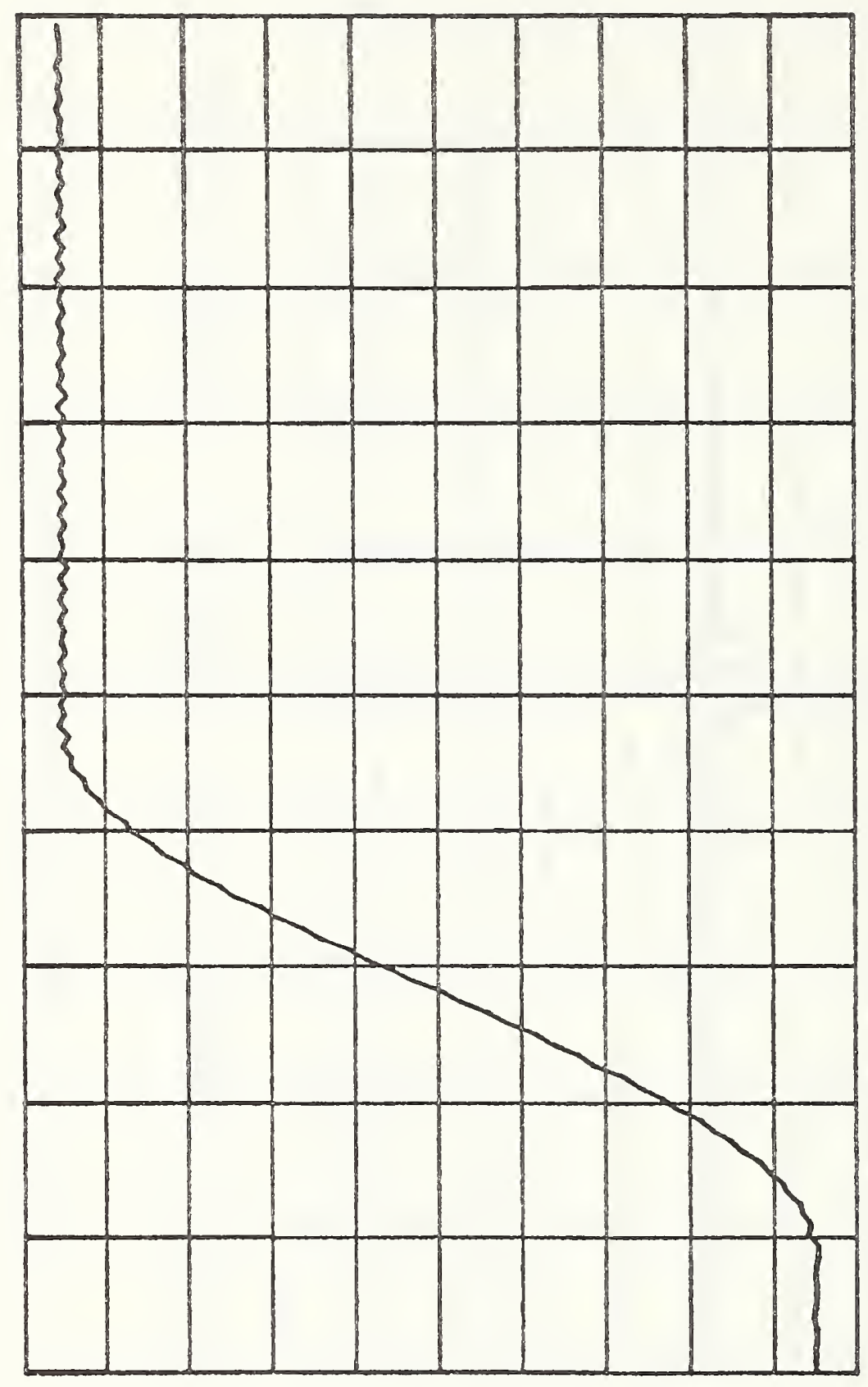

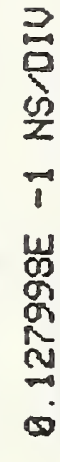

ل1

崩

o

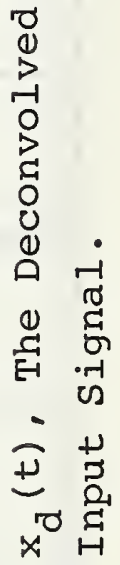

$u$
0
0
0
0
0

نำ

\begin{tabular}{l}
11 \\
0 \\
0 \\
0 \\
0 \\
\hline
\end{tabular} 


\section{SUMMARY AND CONCLUSIONS}

This report was devoted to the study of the theory and application of the homomorphic transformation to time domain spectroscopy and scattering problems.

Chapter 1 was an introductory chapter which reviewed the contents of the work presented in this report.

Chapter 2 started with a review of the convolution relations in both the time and frequency domains and the need for deconvolution (separation) techniques. The general class of the homomorphic systems was defined, and their properties were stated showing how they can be used for signal separations. In particular, the homomorphic deconvolution transform was developed. The related problems, such as the nonanalyticity of the complex logarithmic function and the effect of the linear phase component, were presented and discussed, and solutions to such problems were given. The computational realization of the deconvolution transformation was made possible in the form of digital computer subroutines HDT and HDF.

Chapter 3 presented several classes of time domain measurement problems in the field of linear microwave network analysis. The homomorphic deconvolution transform, that was developed in chapter 2 , was used in deconvoluting various convolutions in these problems. The examples that were considered are:

(1) pure resistive loading along a transmission line,

(2) commensurate transmission line networks,

(3) TDT type measurement and with two reactive discontinuities, and

(4) conventional TDR measurement to determine a reactive load impedance.

The homomorphic deconvolution method was proven to be very effective in separating convolutions. The method was not limited by the overlap of consequent reflections in the time domain.

In chapter 4 the theory of the time domain dielectric spectroscopy measurements was presented. Conventionally this method of measurement works only for nonoverlapping reflections; i.e., reflections occurring in separate time domain windows. If the reflections overlap, only approximate solutions may be applied to the frequency domain form of the signal. By applying the homomorphic deconvolution transformation to the TDDS waveform, a new method was developed which was not limited by overlapping reflections. The new method was applied to the well known Debye dielectric model. This method eliminated the overlapping reflections and determined the dielectric parameters to within $0.3 \%$ error of their model values. The new method is a general one and has great utility for time domain measurements on dielectrics since it does not include any restrictions on the dielectric material parameters or require the assumption of a prior physical model. Furthermore, the new method is not restricted to time domain spectroscopy of only dielectric materials but has utility for time domain spectroscopy in general such as for magnetic and other phenomena. 
Chapter 5 began with an introduction about the role that the time domain measurement techniques can play in solving electromagnetic radiation problems. Then the distributed nature of the expected cepstrum in such problems was discussed and compared with previously studied discrete cases. Two examples of applying the homomorphic deconvolution transform to an antenna system were presented, and the transform proved to be very successful in the removal of the unwanted reflections and scattering components either overlapping or not overlapping the main signal containing the desired signal information.

Chapter 6 was devoted to the modeling of the feed-through sampling-head. The theory and construction of the sampling head were reviewed. The physical dimensions of the structure discontinuities were used to formulate an equivalent network for the sampling-head input and output lines. The dimensions of the biconical cavity together with TDR measurements were used to model the cavity part of the sampler. The dc characteristics of the diode together with TDR measurements vs. sampling diode bias were used to evaluate various diode model parameters. The strobing pulse was measured, and the data corrected for both amplitude and risetime. All of these sampling-head parameters were simulated in the form of a digital computer program which was run to obtain a theoretical estimate of the samplinghead step response. The program was implemented in two steps because of the large number of elements and information required to simulate the sampling system. The step response was found to vary with the diode reverse bias; the higher the reverse bias the faster was the step response, within limits. The step response at the manufacturer's suggested bias and at the bias for the fastest step response was obtained. The homomorphic deconvolution transform developed in chapter 2 was slightly modified in order to be used to deconvolve the sampling-head response contribution from the output of the sampling oscilloscope. Deconvolution of the observed waveform provides an estimate of the actual (true) waveform at the input port of the oscilloscope. 


\section{ACKNOWLEDGMENT}

The first author acknowledges the National Bureau of Standards for providing him with a Guest Worker appointment to work in the Boulder Laboratories under the NBS/University of Colorado Joint Graduate Program in Electromagnetics Metrology, and the cooperation between the Graduate Schools of the University of Toledo and the University of Colorado which facilitated the continuance of the author's dissertation research in Boulder, Colorado.

Also, the first author wishes to acknowledge the invaluable assistance and guidance of his advisor, Dr. N. S. Nahman (Chief, Time Domain Metrology Program, Electromagnetics Division, National Bureau of Standards; Professor, Electrical Engineering Department, University of Colorado; and formerly Professor and Chairman, Electrical Engineering Department, University of Toledo), who suggested the subject of this report and contributed in trany ways to the completion of this work.

As an NBS Guest Worker, the first author had the privilege of doing his research in close association with J. R. Andrews, Ph. D., W. L. Gans, M. S., and A. R. Ondrejka, M.S., each who contributed a stimulating environment for the author's research. Finally, sincere thanks go to Mrs. Sandra McCarthy, Miss Sharon Foote, and Mrs. Sheila Aaker who significantly contributed to the editing and preparation of this report. 
APPENDIX A

THE $z$-TRANSFORM AND THE DISCRETE FOURIER TRANSFORM

The $z$-transform is a generalization of the Fourier transform in the case of discrete time signals (sequences). The $z$-transform of the sequence $x(n)$, where $n$ is the sequence variable, is defined as

$$
z[x(n)]=X(z)=\sum_{n=-\infty}^{\infty} x(n) z^{-n}
$$

where $z[]$ is the $z$-transform operator and $z$ is a complex variable.

The $z$-transform $X(z)$ does not converge for all sequences $x(n)$ or for all values of $z$. The region of convergence, i.e., the set of zvalues for which $X(z)$ of a given $x(n)$ converges, is very important. It must be defined in association with the transform $X(z)$ in order for the inverse transform to be uniquely defined. Generally speaking, the $z$-transform, eq. (A.1), converges in an annular region of the z-plane given by

$$
u \leq R_{-}<|z|<R_{+} \leq \infty
$$

For a stable time domain sequence, the convergence region of the z-transform includes the unit circle $|z|=1$.

The inverse $z$-transform is given by

$$
x(n)=\frac{1}{2 \pi j} \oint_{C} x(z) z^{n l} d z
$$

where $C$ is a counterclockwise closed contour in the region of convergence of $X(z)$ encircling the origin of the z-plane.

If the complex variable $z$ in eq. (A.1) is expressed in the polar form

$z=r e^{j \omega}$

the $z$-transform will take the form

$$
x\left(r e^{j \omega}\right)=\sum_{n=-\infty}^{\infty} x(n) r^{-n_{1}-j \omega n}
$$

which can be viewed as the Fourler transform of $x(n)$ multiplied by an exponential sequence $r^{-n}$. If $r=1(|z|=1)$ the $z$-transform becomes identical to the Fourier transform of the sequence,

$$
X\left(e^{j \omega}\right)=\sum_{n=-\infty}^{\infty} x(n) e^{-j \omega n} .
$$

For finite duration sequences, with total number of samples $\mathbb{N}$, the $z$-transform, eq. (A.1), will take the form

$$
X(z)=\sum_{n=0}^{N-1} x(n) z^{-n}
$$


$X\left(e^{j \omega}\right)=\sum_{n=0}^{N-1} x(n) e^{-j \omega n}$, on the unit circle.

For computational reasons, only a finite number of samples of the z-transforms can be calculated. Sampling the z-transform at $\mathrm{N}$-equally spaced samples on the unit circle $|z|=1$ results in the discrete Fourier transform DFT,

$$
\begin{aligned}
x(k) & =\left.x\left(e^{j \omega}\right)\right|_{\omega}=2 \pi k / N \\
& =\sum_{n=0}^{N-1} x(n) e^{-j(2 \pi k / N) n}
\end{aligned}
$$

where $\mathrm{k}$ is a sequence variable that takes on values from. 0 to $\mathrm{N}-1$. By this sampling process the finite duration sequence $x(n)$ is forced into being periodic with period $N$. The transform $X(k)$ will also be periodic with the same period. The discrete inverse Fourier transform DIFT is given by

$$
x(n)=\frac{1}{N} \sum_{k=0}^{N-1} x(k) e^{j(2 \pi k / N) n} .
$$

Both the z-transform and the DFT have the following listed properties even though these properties will be presented only in the z-transform form. It is to be remembered that the DFT corresponding relations can be obtained by replacing the complex variable $z$ by $e^{j(2 \pi k / N) n}$ and the operator $z[]$ by DFT[ ]. The properties are:

1. Linearity

$$
z[a x(n)+b y(n)]=a X(z)+b Y(z) .
$$

2. Sequence shift

$$
z\left[x\left(n-n_{0}\right)\right]=z^{-n_{0}} x(z) \text {. }
$$

3. Convolution

$$
z\left[x_{1}(n) * x_{2}(n)\right]=z\left[x_{1}(n)\right]: z\left[x_{2}(n)\right]
$$

Finally, one additional but very useful property of the z-transform will now be discussed; it is the multiplication by an exponential sequence. This property is

$$
z\left[\alpha^{n} x(n)\right]=x(z / \alpha)
$$

The importance of this property lies in its ability to scale the convergence region by the factor $1 / \alpha$, thus enabling the inclusion of the unit circle in the convergence region and, consequently, stabilizing unstable sequences. This method can also be used to remove the transform zeros that may lie on the unit circle giving rise to singularities upon taking the complex logarithm. This method of scaling the z-transform is often called the exponential weighting. 
SUBROUTINE HDT $(X, H, N, N S H)$

C**** THS MOMOMORPHIC DECONUOLUTION TRANSFORM

C*X** FOR TIME DOM SIGNALS COMPLEX $X(1), H(1), C L X, C C$

C**** TAKE THE FOURIER TRANSFORM

CALL FFT (X,N, TRUE.)

C***** CALL SUBROUT INE HOF CALL HOF (X.H,N.NSH)

C*N** IMUERSE FQURIER TRANSFORMS OF SEPARATED COMPONENTS CALL FFT (X,N, FALSE.) CALL FFT (H.N, FALSE.) RETURN END

SUBROUT INE HOF $(X, H, N, N S H)$

C*A+* THE HONOMORPHIC DECONUOLUTION TRANSFORM

C**** FOR FREQ DOM SIGNALS

COMPLEX $X(1), H(1), C L X, C C$

$P I=4$. WATANK 1.8)

$N 2=N / 2+1$

C*N** CONTINUOUS COMPLEX LOGARITHM

$C=0.0$

$A R X=0.0$

DO $20 L=1, N 2$

$A R S=Q R X$

$C C=X(L)$

CALL CLG (CE, CLX)

$A R X=A T \operatorname{maS}(C X)$

$A R=A R X-A R S$

IF (AR.GT.API) $\mathrm{C}=\mathrm{C}-2$. $\mathrm{API}$

IF (AR.LT. $-P I) C=C+2$. $* P I$

$C C=C \sin (0.0, C)$

$X(L)=C X+C C$

20 CONTIMUS

CW LINEAR PHASE ELIMINATION

NSH AIMACS X(N2) ) PI

TYPE " SEQ SHIFTED" NSH, " SAMPLE"

$0045 L=1, N 2$

$45 X(L)=K(L)-C T P L X 0.0,2 . *(L-1)$ KSHAPIN) 


\section{$N 3=\sqrt{2}-2$}

DO 25 L=1,N3

$25 X(N 2+1-1=C$ ONJ $J(X(N 2-L))$

CWOW INUERSE FOURIER TRANSFORT

CQL 1 FFT $(X, N$, FALSE.

TYPE " CEPSTRUM"

* * SEPARATION AND INUERSE PROCESSING

$C A L S_{S P}(X, H, N, N S H)$

RETLRN

END

SUBROUTINE FFT $(X, N, F !)$

C**** FRST FOURIER TRANSFORM : FI=.TRUE.

C**** INUERSE TRANSFORM: FI=.FALSE.

COMPLEX X(N),U,W,T

LOGICAL FI

$E N=N$

$M=R$ OG $(E N), A L O G(2)+$.

$N 2=N / 2$

$\mathrm{NI}=\mathrm{N}-1$

$j=1$

$007 I=1, N !$

IF (I. (E. J) CO TO 5

$T=x(j)$

$x(j)=x(j)$

$5 x=12$

$X(I)=T$

6 If $\langle K . G E . J\rangle$ GO TO 7

$J=J-K$

$K=K / 2$

7

GD TO 6

$j=4 k$

$P I=4$. *ATANK I.B)

DO $20 \mathrm{~L}=1, \mathrm{M}$

$L_{2}=2 \times 2 \times$

$L i=L 2 / 2$

IF $\{L 1 . E R .1\rangle$ CO TO

$U=(\$ ., 0$.

$0=L 1$

IF (.NOT .FI) $D=-D$

8

$W=\operatorname{cin} Q x(\cos (P I / D),-\operatorname{SIN}(P I / D))$

Do 28 Ji,L1

DO 10 I $=J, N, L 2$

$I F I=I+L !$

$T=X(P\lfloor)$

If (J.AE.1) T=T䊉

$X(I P I)=X(I)-T$

$18 X(I)=X(I)+T$

28 CONTINUE

NE. 1) UEUWWh

IF (FI) GO TO 40

$0030 \quad I=1, N$

$30 X(1)=X(1)$ VN

40 RETLRK

END 


\section{SUBROUTINE SEP $(X, H, N, N S H)$}

CN** SEPARATION OF CEPSTRUM COMPONENTS AND INUERSE PROCESSIMG COMPLEX X(1), K 1$),$ CC

DIMENSION NS(5), NR(5), RX(1024)

LOCICA MPF. IS

PI =4. WATAN I. O)

$0040 \mathrm{~L}=1, N$

$40 \operatorname{RX}(L)=R E A(X(L))$

C**** LINEAR FILTER ( SEPARATION PROCESS)

30 TYPE " READY FOR SEPARATION

ACCEPT "NLMBER OF ELIMINATEO REGIONS $=$ ?

DO $5 I=1, N Z R$

ACCEPT "ELIMINATE POINTS STARTINT AT:

5 ACCEPT

AND ERDING AT :

NS(NZR+1) $=N+1$

HOF $=$. TRUE.

NS1 1 NS 1 ) -1

$900010 \mathrm{~L}=1, \mathrm{NS} 1$

$X(1)=C H P L X(K X(L), 0.0)$

10 If $(\mu P F) X(L)=(0.0,0.0)$

DD $16 I=1, N Z R$

NSI $=N S(I)$

$N F I \equiv N F(I)$

OO 12 IINI, NFI

$X(L)=0.0$

12 IF (MPF) $X(L)=R X(L)-X(L)$

NFII $=4 F(I)+1$

NSII $=N 3(I+1)-1$

o0 14 Laxif I1.NSI

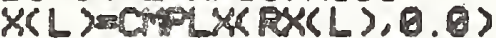

14 IF ( $M P F)$ XS $)$ ) $(0.0,0.0)$

16 CONTIMY

CW* IMUERSE PROCESSING: FOURIER TRANGONM

CALL FFT (X, N. . TRUE.)

C*** RETAIN LINEAR PHASE ANO COMPLEX EXPONENTIAL

nO $20 \mathrm{~L}=1, \mathrm{~N}$

$C C=C$ CPI $X(0,0,2 * *(L-1) * N S H * P I / N)$

IF (.NOT. HOF) X(L) $\times X(L)+C C$

20 CONTINU

$X(L)=C \exp (X(L))$

IF (. NOT. HPF) 60 TO 99

C*W HPF FREQ DON SIGNAL

$70 \mathrm{~B}=1, \mathrm{~N}$

CX*** REPEAT FOR LPF COMPONENT

HPF $=$. FALSE.

CO TO 9 S

99 ACOEPT "ANOTHER SEP TRIAL ? -1 IYES, QAND

"A, NZR

"N, NS(I)

"NF(I)

IF (IS) CO TO 30

RETURN

END 
C**** PROGRAM TONA : TIME DOM NETWORK ANOLYSIS

C**** THIS PROGRAM CAN HANDLE ANY OF THE FOLLOWING NETWORK ELEMENTS : C**** I: CUR SRCE. R: RES, D: DIOOE (NLR), C: CAP, $N$ : NL CAP,

C*** U: ULTG SRCE, L: IND, T: TRANSM LINE

DIMENSION C1(651,11),C1T(11),C2(651,11),C2T (11), ROK 11), NT(11)

DIMENSION IBNCHK 7 , 100 ), ELUAL (109), NODOUT $(2,30$ ), UOUT ( 39 )

DIMENSION TIME(651), TOU 651, 1), TOAK 1)

DIMENSION G(35, 35), C (35), U( 35), USAK 35), NU( 19)

DIMENSION UPLOT $(651,26)$

COMMON MLHPTS. TMIN, TMAX

$N D I M=35$

$M=0$

C**** READ INPUT DATA

CALL READ IBNCH, ELUAL, NEL, NY, NODOUT, NOUT, RO, LL, NN)

$T=$ TMAXNUMPTS

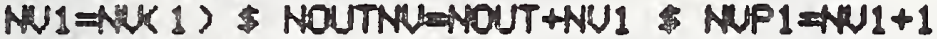

$U L=2 * L L$ NOUTLL $=$ NOUT $+L L L$

MUPTS AMMTST 1

DO 2 Je1, NUMPTS

TOUK $J, 1>=0.25$

$2 \operatorname{IIME}(J)=(J-1) * T$

TOK 1,1$)=0.0$

IS $=1$

CALL PLOT (TIME, TOK 1, 1), MUMPTS, 1GHINPUT US T)

C* ADJUST ELEMENT LIST

CALL PREPARL IBNCH, ELUAL, NEL, NN,T,NT,RB)

DO $18 \mathrm{~J}=1, \mathrm{NN}$

USAK J $=0.8$

18 K J $)=0$

DO 20 Lख1,LL

CITCL XO

20 CETCL 2209.

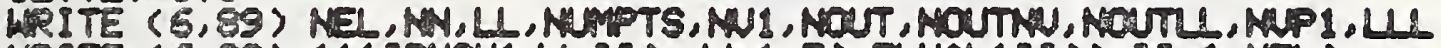

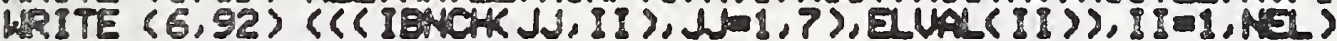

DO 50 Ans 1, MMTS

CX*** SET UP NOOE EQUATIONS

DO 22 II $=1$, IS

22 TONAK II $D=$ TOUKN, II)

24 CALL NOOEQ (IBNCH, ELUAL, NEL, G, C,U, NN, NDIM, USAN, TIME(N), T, TOUN, $x$

C1T,C2T)

IF (N.GT.1.OR.M.GT.1) GO TO 28

WRITE $(6,92)$ ( ( (IBNCAK JJ,II ), JJ=1,7), EL UAL (II )), II =1, MEI)

$0026 I I=1, N$

26 WRITE $(6,91)([X I I, J J), J J=1, N W), C(I I), U(I I)$

28 CONTINUE

C**** SOLVE MOOE EQUATIONS

CAL SLNEQ (NN, NOIM, G,C) 
C*** U(J)-Ci」) IS THE INCREMENT IN THE JTH NODE UOLTAGE $E R R=9.0$

$0030 j=1, N$

$30(x J)=c(J)$

$E R R=E R R+(U(j)-C(1)) * * 2$

$M=x+1$

CW TEST CONUERLENCE CRITERION

IFCERR.GT.1.E-6.AND.M.LT.15 >60 TO 24

WRITE $(6,93) N$, U 8 )

CAL OUTPUT (NODOUT, NOUTLL, U, VOUT)

DO $32 I I=1$, NOUT

32 UPLOTSN, I I IUOUTCII)

$0034 J j=1, N \cup 1$

$I I=A O U T+J I \& N J=N(K J J+1)$

34 UPLOT(N, II ISUKNUJ)

$0036 \mathrm{~L}=1, \mathrm{LL}$

II ANOUTMHAL \& JjaMOUTH

36 UPLTCN. II ) UOUTC JJ)

Cow STON TROASMISSION LINE CURPENTS

$0938 \mathrm{~L}=1,11$

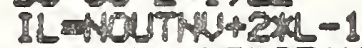

CI $N, L$ )APLOT (N, IL ) RPX L )-CZT(L)

$36 C 2(N, L)=(M, O T(N, I L+1) / R O R(L)-C 1 T(L)$

CW STORE NODE VOL TAGES FOR UPOATING CAPACITORS

$0040 \mathrm{Jz} \perp, \mathrm{MN}$

40 USAUK $J)=(K J)$

$M=0$

DO $45 L=1, L L$

IF (N.LT.NTRL) ) 60 TO 50

CX* UPDATE TL OELAYED CURRENT SOURCES

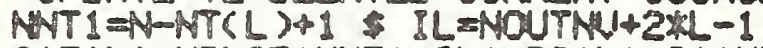

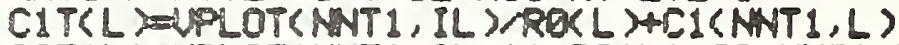

45 C2T $(L)=U L O T(N N T 1, I L+1) / R Q R(L)+C 2(N N T I, L)$

59 CONTINUT

DO 55 II 1 , NOUTN

ITITL $=I T$ (II, NOUT)

55 CALL PLOT (TIME, LPLOT 1, II), MNPTS, ITITL)

WRITE $(6,94)$ TMAX

IF (NOUT. EQ.O) GO TO 65

WRITE $(6,95)(\omega,($ NOOOUT $(I I, U), I I=1,2), \omega=1$, NOUT)

$0060 \mathrm{Na} 1$. MMPTS

TNR= IIME(N)/TMAX

60 WRITE $(6.91)$ TNR, (UPLOT $(N, 11), I I=1$, NOUT)

65 IF (MMI.PQ. 8 ) 60 TO 90

If (NOUT.NE. (2) WRITE (6,94) THAX

WFIT $(5,96)$ (NKII), II=2, MPI)

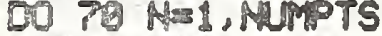

THRETIME(N)/TMAX

70 WRITE (6.91) TNR, (UPLOT(N,II HOUT), II I, NI)

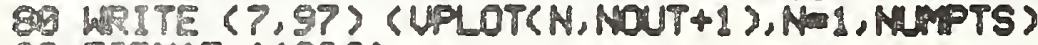

23 Format ( 1916 )

90 FOMPAT (BF 1 .

91 PDPAT (3X, 10. 13.7 )

S2 FOMAT (1X,A4, GI3,E15.5)

93 POAMT ( 97, E15.9)

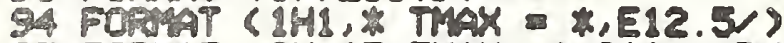

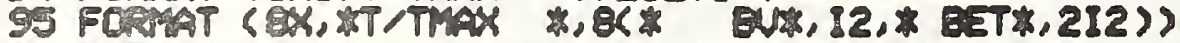

96 FOMAT $(*$ T TMAK $*, 9(*$ MOOE $*, 13)\rangle$

97 FORMAT (SE16.9)

STOP

END 
C**** PROGRPM SMPL: SAMPLING-HEAD STEP RESPONSE

DIMENSION IBNCH 7,42 ). ELUAL (42)

DIMENSION $(16,17),(216), U(16)$, USAU $(16)$

DIMENSION TOU 500,2 ;, TDUN 3 ;, TOUS( 500 )

DIMENSION CI(590), C2(500), UPLOT(509,2)

$M=0$

C**** READ INPUT DATA

TYPE "INPUT : NEL, NN. NPTS, NUMPTS, NT, T, RO, NBB, N11, N21, N12, NE2"

ACCEPT NEL, NN, NFTS, NUMPTS, NT, T, RQ, NBB, N11, N21, N12, N22

ACCEPT "INPUT : NBEN. NC. UO, TOUN 3) "NBEN, NC, UO, TDUK 3)

READ $(13,92)$ ( $(I B N C H K(I, J J), I I=1,7), E L$ UAL $(J), j J=1, N E L$ )

WRITE $(10,92)$ ( IBNCHK II, JJ),II=1,7), ELUAL $(J j), J J=1, N E I$ )

READ $(13,97)$ (TDU $J, 2), j=1$, NPTS)

REAO $(13,97$ ) (TOK 1,1 ), J=1. NUMPTS)

TOU2F = TDU $N P T S, 2$ ?

OO $4 \mathrm{~J}=1$, MUMPTS

TDK $J, 1)=\operatorname{TOU}(j, 1) / 25.0$

IF (J.GT.NPTS) TDU J, 2 ) $=$ TDUZ

4 Tous $j$, $=$ TOUK $(j, 2)$

DIIU $=$ EI UAL (NBB)

02 I $=E L$ UAL $(N B B+1)$

ACCEPT "FEED BACK FACTOR = ",FBF

NSTP $=1$

NPTS $=250$

DO 80 NSPE=NSEN, NUMPTS, NSTP

NDENSPE-NOTS

IF (ND.EQ.QS) 60 TO 16

DO $8 \mathrm{~J}=1, N O$

8 TDX J,2)=TDUS 1 1)

DO 10 Ja1, NPTS

10 TaK $J+N 0,2$ 2atous $J)$

16 CONTINUE

DO :8 $J=1, N$

Usax J $=0.0$

$18 \cup(J)=0$.

EL UAL ( NS8 ) $=0$ I IU+TDUN 3)

ELURL $(N 38+1)=02 I U-T D U N 3$ ) 21 . ES

$C 1 T=0$.

C2T $T=0$.

DO $50 \mathrm{~N} 1$, NSPE

C**** SET UP NDOE EQUATIOAS

TOUN : $=\operatorname{TDK} N, 1$ )

TOAN 2 I $=\operatorname{TON} N, 2$ )

TIME $=(N-1) 2 * T$

24 CALl NOTEQ (IBNCH, ELUAL, NEL, G, C, U, NN, NOIM, USAN, TIME(N), T, TDEN, $X$ CIT,C2T)

C**K* SOLUE NODE EQUATIONS

CAL SLNEQ (NN, NOIM, G,C)

C**** $(K J)-C(j)$ IS THE INCREMENT IN THE JTH NOOE VALTACE

$E R O=0.0$

DO $301, N N$

30

$E R O=E R O+(J(X)-C(j)) * 2$

$(x J)=(J J)$

$M=1+1$ 
C* TEST CONUERGENCE CRITERION

IFCERO.GT.1.E-6. AND.M.LT.15)EO TO 24

URLOT $(N, 1)=U(N 11)-U(N 21)$

UPLOT $N, 2$ ) $=U(N 12)-U(N 22)$

CWW STORE TRANSMISSION LINE CURPENTS

CISN)=UPLOT $(N, 1) / R O-C Z T$

CE(N)AUPOT $N, 2) / R O-C I T$

C*** STORE NODE UOLTAGES FOR UPDATING CAPACITORS $0040 \mathrm{~J}=1, \mathrm{NN}$

40 USAUK $J$ JaUK $(J)$

IF (N.GT.I) GO TO 42

$42 M=8$

UCI I $a$ U $(2)-4 \times 1$ )

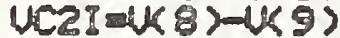

IF (N.LT.NT) CO TO 50

C**** UPDATE TL DELAYED CURRENT SDURCES

NWT $1=N-N T+1$

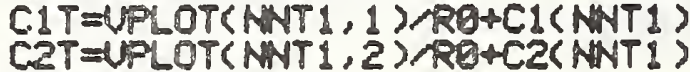

50 CONTINUE

USC $=(4 \times 8)-(k 9))-U C 2 I-(U(1)-U(2))-U C 1 I$

$\mathrm{MC}=\mathrm{NC}+1$

TaAk 3) $=$ TOUNK 3)+FBF*USC

$N C T=(N C-1) * T / 1 . E-12+.5$

No= UO+USC

WRITE (10.93) NC, NCT, NO, TOAK 3)

SO CONTINLE

92 FORMAT ( $1 X, A 4, I 6,513, E 15.5$ )

93 FORMAT (1X,15, 5X, 15, 2X, "PS", 2E17.6)

97 FORMAT (5E16.9)

STOP

END 
SUEROUTINE READ IBNCH, ELUAL, NEL, NH, NODOUT, NOUT, RQ, LL, KN) DIMENSION IBNCH $(7,1)$, ELUAL 1 ;, NODOUT $(2,1)$, OUMTY $(15)$, RQ 1 ) INTEGER TA, NU( 1), II(25), NUT (10), NUA 9 )

COMTON NUMPTS, TFMIN, TFMAX

DATA IBLAWK IH, NUA, \$HA, 1HB, IHC, 1HD, 1HE, IHF, 1HE, 1HH, 1HI/

C**** READ AND WRITE NOME CARO

REAO $(5,50$ ) (DUMMTY $(J), j=1,15$ )

LRITE $(6,52$ ) (DUATY $(j), j=1,15$ )

C**** REOD NEXT ELEMENT

5 NEL $=N E L+1$

REAO $\langle 5,54$ ) (IBNCK J, KEL ), J=1, 5), ISGN, IOUT, IS, (DUMTYK $J$ ), J1, 2),

$X$ (NUT $(J), J=1,18$ )

C*A** REAO FIRST CHARACTER OF NAME ? =ELEMENT TYYPE)

BCCKSPACE 5

$R \leq A D\langle 5,56\rangle$ TA

C* DECOOE ELEMENT TYPE

NTYPE=NDODE $T$ TA

IF (NTYPE-9) $20,35,10$

CW SAE GENERAL INFORMTION CARO DATA

10 MUP IS IBNCK $4, N E$ ?

TFMOX $=$ OUMTY (1) \& TFMIN=OUMTY(2)

IBNCKX I, NEL $2=4 H$

$\mathrm{NEI}=\mathrm{NE}-1$

$0015 \mathrm{j}=1,18$

15 MX J) $=N U T\langle J\rangle$

NUI $=N$ K 1 ?

If (NU1.EQ.9) 60 TO 5

WRITE $(6,59)$ (NUK J十1), Ja1, NU1)

WRITE $(5,69)(M U A X), j=1, N U 1)$

30 ELUAL(NEL ) QUMHYS 1)

C* STOAE TYPE OF ELEMENT

IENCK G, NEI LANTYPE

IBNCK?, NEL $)=0.8$

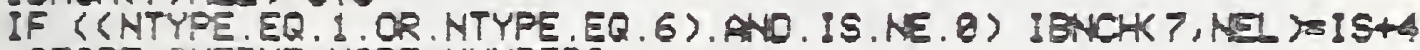

C*** STORE OUTPUT NOOE NUMBERS

IOUT $=$ NCOOE ( IOUT)

IF (IOUT. Ne.6) CO TO 39

NOUT $=$ NOUT +1

C*** CHECK SIGY OF OUTPUT VOLTACE

IF (ISCH. EQ. IBLANK) CO TO 25

NOOOUT 1 , NOUT 2 = I BNCK S, NEL)

NOOOUT ( 2, NOUT IEIBNOK 4 , NEL)

60 TO 30

25 NOOOUT (1, NOUT ) = IBNCK 4, NSI?

NOOOUT ( 2, NOUT ) =IBNCH S, NEL)

C * STORE HIGHEST NOOE NUMER

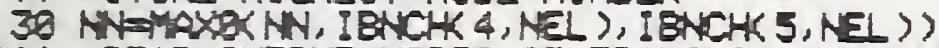

CWN* READ OUTPUT NODES OF TRANSMISSION LINE

IF (IBACH 6,NE1).NE.8) GO TO 5

$L=L+1$

$R S A O(5,62) N 3, N 4, R O R L)$

$N N=M a x \times N(N 3, N 4)$ 
IBNCHK 2, NEL I $=N 3$ IBNCHK 3, NEL X XNA4

II L ) $\approx$ N

60 TO 5

35 IBNCHK 1, NEL ) $=44$

MEL $\Rightarrow$ NEL -1

$\mathrm{L}=\mathrm{L}$

$0040 \mathrm{~L}=1, \mathrm{LL}$

$I \perp L I I\langle L\rangle$ IL $=N O U T+2 * L-1$

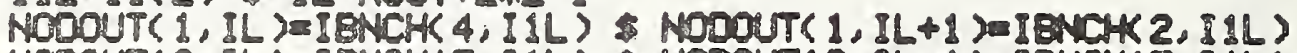

49

NODOUT $(2, I L) I B N C K 5, I I L)$ MOOOUT $(2, I L+I$ ) IENCKK $3, I I L)$

CONTINUE

WRTE $(6,64)$

$L=8$

00 4S J I. NEL

WRITE $(6,66)$ IBNCK I, J), IBNCK $4, J)$, IENOK $5, J)$, ELALL $(J)$,

$x$

IF (IBACHK 6, 1$)$. EQ.8) WRITE $(6,68)$ IBNCHK $2, J)$, IBNCHK 3,1$), R Q(L+1)$

45 CONTINUE

RETUNON

58 FOFMAT (15A4)

52 FORMAT (18K 1H*), 1594,10(1H*))

54 FORMOT (A4, A1, A4,I4,I3,IX,A1, A1, I1, 2E15.5,1013)

56 FOMMAT (A1)

58 FORMAT ( $/$ * QUTPUT NOOES AND THEIR CORRESPONOINC ALPHABETIC *, $x$ WMATS USEO IN MICROF ILM PLOTSK, $1 X, x 2 X, I 3)$ )

69 FORMOT $(1 X, 9(4 X, A 1))$

62 FOPMAT (10X, 2I3, 3X, E15.5)

64 FOMMAT (, S BRANCH NODE $x$

* MONE MOS.

ELEMENT

WALUE

66 FOPMAT (1X,AA, 2I3,E15.5, 1X,A4, 3X,A1)

68 FORMAT ( $5 \times, 213, E 15.5)$

END

SUBROUTINE PREPAR( IBNCH, ELUAL, NEL, NN, T, NT, RO)

OIRENSION IBACH ?, 1), ELUAL (1), NT (1), RER 1)

CONWON MMPTS, TMIN, TMAX

DATA IQLAK /IH / IREF/IHI/

C*** MATCH CONTROLLED RND CONTROLLING ELEMENTS

DO 2 I I, NEL

If (IBNCHK $6, I$ ) . NE. 1) 60 TO 2

IF (IBNCK 2, I).EQ. IBLAMK) GO TO 2

IF (IBNOKK 2, I).EQ. IREF) 60 TO 2

DO \& Ja1, NEL

IF (IENCHK 3, I). NE. IBNCH 1, J) CO TO 1

C* * STORE CONTROLLING NODE MUMBERS FOR USE IN NOOEQ

IBCHK 2, I IEIBNCKK 4,J)

IBNCK 3, I $2=I B N C H K 5, J)$

1 CONTINU

2 CONTINUE

NEL $1=N 5$

CW MODEL INOEPENOENT VOLTAEE SOURCE BY

C GYRATOR AND INDEPENDENT CURRENT SOURCE

003 I 1. Nel.

If (IBNCK 6. I) .NE.6) CO TO 3 
IF (IBNCHK 2, I). NE. IBLANK) 60 TO 3

CALL GYRATRK ISNCH, ELUAL, NEL, NV, 1, 1.0,1.8)

WW MOUE AND CHANGE TYPE OF INDEPENDENT SOURCE IBNCH 4, I Y =NH

IBNOHK $5, I>=0$

$4 \times 6,2=1$

3 COATINHE

CW*W* HODEL INOUCTOR BY GYRATOR ANO CAPAC!TOR

$00 \mathrm{~S} I 21, \mathrm{NEL} 1$

IF (IBNETK S, I) NE.?) CO TO S

CAU ETRATR, IBNCH, EL UAL, NEL, N, i, 1. O, 1.8)

CA MOUE AWD REPLACE INDUETOR WITH CAPACITOR

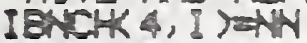

IBNCAK $(5, I)=0$

5 CONTINUE

CXWK* MOOEL SHDRT CIRCUIT BY A GYRATOR AND OPEN CIRCUIT

$006 I=1, N E L !$

IF (IBACHK 6, I) NE. 2) GO TO 6

If (ELUALCI) NE.0.0) 00 TO 6

6 CONTIMUE

CALL SCET (IBACH, EL UR, NEL, NV, I)

C**** REPLAE TRANSMISSION LINE BY ITS MOOSL

$L=0$

DO $7 I=1, N E L 1$

IF (IBNCK 6, I). NE.8) IS TO ?

$L=-1$

NT(L)=EL $(40)(I)>T+8.5$

CALL TRLIN (IBNCH, ELUAL, NEL, I, Rg(L))

7 CONTINUE

CWN MOOEL NONLINEAR CAPACITOR BY ITS COMPANION MOOEL

008 $8=1, N E 1$

IF (IBNCHK 6, I).NE.5) 60 TO 8

$T I=1 . / T$

CALL GYRATRK IBNCH, ELUAL, NEL, NY, I, T1, 1, 8)

IBNCK $?, N E-1$, $=1$

CWW CHANCE TO VARIABLE CLRRENT SOLCE

C NOTICE SIGN CHANGE IN NOOEQ

IBNCH 3 , I IENA

IENCHK $G, I\rangle=1$

ISACK ?, I $2=1$

C*K** AOO LARIABLE CONDUCTANCE AND UURENT SOURCSS

NEL XNEL +1

IBNCHK 1, NEL 2aIENCHK 1,1 )

IENCH 2, NEL $2=1 \mathrm{H}$

ISACH 3 , NEL $>$ I

IBACK $4, N E L$ IDNW

IExak $5, N$, $2=0$

IBt.H 6 , hes $>202$

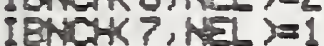

$N E L=N E L+1$

(BACHK 1, NEL ) = IBNCK 1, I)

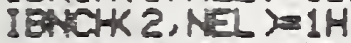

$\operatorname{IBNCK} 3, N$ EI $=N \mathrm{~N}$

IBNCHK 4, NEL J $=N$

I 3 CK $5, N$ E $2=0$

ISNCK $6, N 51$

IBNDK $7, N$ I $=2$

8 CONTINUE

RETLN

ENO 
X

SUBROUTINE NOOEQK IBNCH, ELUAL, NEL, G, C, U, NN, NOIM, USAU, TIME, T, TDUAL,

REAL LAMDA

OIMENSION IBNCHK 7, 1), ELUAL (1), TDUAL (1), C1T(1), C2T:1)

DIMENSION G(NOIM, 1), C (1), UK 1), USAK 1)

COMUN AUMPTS, TMIN, TMAX

DATA IBLANK/IH I INIT/OR, IREF/IHI/

DATA CSAT/2.76E-11/, LAMOA/38.61/

C*** ZERO NOOE MATRIX AND CURRENT VECTOR

oO $5 k=1, N$

$C(K)=0.0$

DO $5 \quad j=1$, AN

$5(K J . K)=0.0$

$L=0$

DO 80 JN N NEL

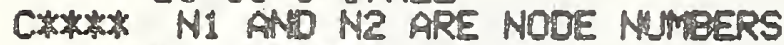

$N 1=I$ BNCH $4, J)$

$N 2=I B N A K 5, j)$

$B U=0.0$ \&UN=0.0

IF (N1.EQ. GO TO 10

$B(1=U K N I) \&$ BUNI=USAUKNI)

10 IF (N2.EQ.O) GO TO 15

$B U=8 U-U N 2) \& B(N=B(N-U S A K(N 2)$

15 CONTINUE

CW DETERMINE ELEMENT TYPE

NTYPE: ISHCHK $5, J)$

60 TO (20, $25,70,75$ ), NTYPE

C* TEST TO SEE IF IMOEPENOENT CURRENT SOURCE

20 IF (IENCHK $2, J)$. EQ. IBLANK) CO TO SS

C*W* MOOIFY NONLIMEAR COPACITOR MOOEL AT DC

IF (TIPE. EQ.Q.Q. ANO. IENCHK 7, J).EQ. 1) CO TO 80

C*

CAL MCCS N1, N2, IBNCHK 2, J), IENOK 3,J), EL UAL $(J), G, N D I M)$

60 TO

CX LOAO CONOUCTANCE INTO G

25 IF (IENCKK 7,1$)$. EQ. Q) GO TO 30

$I C N=\operatorname{IBNCK} 3, J)$

NCI=IBNCHK 4, ICNL) \$ NCZ=IBNCKK S, ICNL)

BUC $=0.0$

IF (NC1. NE.O) BUC=UKNC1)

IF (NC2. NE. B) BUC=BUC-U(NC2)

EL UAL $(J)=C N L$ ( BUC)

$30 \operatorname{CONO}=1$. R ELUAL $(J)$

CALI BNCHKN1,NE, COND, G,NDIM)

60.7088

35 IF (IBNCH ?, J).GT. 4) 60 TO 60

ICNT $=$ IBACH $3, d$ )

60 TO $(65,49,45,59,55)$, I BNCKK $?, j)+1$

CXNOW MOOIFY MC MOOEL AT OC

40 IF (TIME. EQ.O.Q) CO TO 80

CW CHOMTE SIGN AS MENTIONED IN PREPAR

ELAL(J)-USARK ICNT ) T $\$$ CO TO ES

45 ELUAL( J ) WUC-CONDWU(ICNT) \& CO TO 65

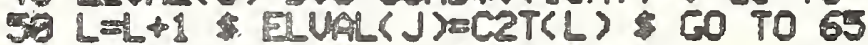

55 ELUAL $(J)=C I T R L) \&$ CO TO 65

60 ISEIENCHK $7, J)-4$ ELUAL( J) TOUAL(IS)

65 CAL SRCE(N1, N2, ELUAL $(J), C)$

60 TO 80

CXOW OEFINE DIODE PARAMETERS

CWOW APPLY INITIAL DIOOE IF ON FIRST ITERATION

70 IF (INIT. EQ.0) BUE ELUA(J) 
IMPSE HEURISTIC LIMITATION OF .8 VATS ON DIOOE GW $B U=A M I M 1(Q 4,0.8)$

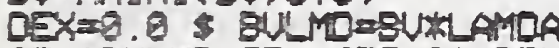

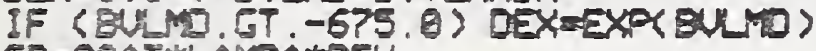

COACSAT WLATOAXDEX

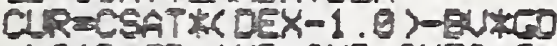

C* * LOAO CO AND CUR INTO E AND E

CALL GHOKNI, N2, GD, G, NOIM)

CALI STCE (N1, N2, CUR, C)

\section{CO TO 89}

C**** FORM COMPANION MODEI OF CAPRCITOR; SKIP IF DC

75 IF (TIP.EQ.8.8) 60 TO 89

$G C=E L(Y A L(J)>T$

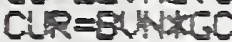

28

CALL ENCKN1, N2, GC, G,NOIM)

CALL SRCE $N 2, N 1$, CUR, C)

CONTINUE

INIT $=1$

RETURN

END

\section{SLEROUTINE SLNEOX $N, N D I M, A, B$ )}

C* * SQLUTION OF N LINEAR EQUATIONS A I, J)*B(J)EB(I)

DIMENSION ANOIM,1),B(1)

$N 1=N+1$

Do $10 \sum=1, N$

$10 A I, N I=B(I)$

DO $48 \mathrm{~K}=1, \mathrm{~N}$

$K !=K+1$

$G=8.8$

$0020 I \approx K, N$

$P=A B S(A I, K)\rangle$

IF (P.LE.G) 60 TO 20

$\Leftrightarrow$ LEI

29 CONTIME

$\infty 25 \mathrm{JN}, \mathrm{NI}$

$G=\alpha L, J\rangle \& \alpha(L, J \nu=K K, J) \$ R K, J=G$

25 contilu

$P=(K, K)$

$0038 J=K 1, N 1$

$32 A K K, J)=A K, J 2 / P$

0040 IaI, N

IF (I.EQ.K) 60 TO 40

$P=-A(I, K)$

DO 35 Jek1, N1

$35 R I, J I=A X I, J\rangle+P * A(K, J)$

48 CONTINUS

DO $45 \mathrm{~J}=1, N$

45 E(J) $\operatorname{sex} J, N I)$

RETLRN

END 
SUBROUTINE GYRATRK IBNCH, ELUAL, NEL, NN, I, E1, G2)

DIMENS ION EL UAL (1), IBNCH 7,1 )

C**** THIS SUBROUTINE GENERATES TWO VCCS'S TO SIMULATE GYRATOR $N I=I$ BNCH 4,1 ) $\$ N 2=1 B N C H K 5, I)$

$M N=N M+1$

C*W* AOD FIRST UCCS

$N E L=N E L+1$

IBACH I, ARE ) IBACK 1.I)

IENCK $2, N E$ I $=N$ N

IBNCH $3, N=1$ : $=0$

IBNCKK $4, N E L=N 1$

IONCHS,$N E$ I $=N 2$

ISACH $6, N E L)=1$

IBNCHK?, NEI $>=0$

ELUAL (NEL ) GI

C*** AOD SECONO UCCS

$N E L=N E L+1$

IBNCHK 1, NE J J IBNCH $1, I$ )

IBNCHK $2, N E$ L $)=N 1$

IENCHK $3, N E L$ ) $=N 2$

IBNCHC $4, \mathrm{Ne}-\mathrm{L}=0$

IBNCKS 5, NE ) $=N$ N

ISNCKR $5, N$ L $>=1$

IEXCK ? NEL 200

EL UAL (NEL) $=62$

RETURN

END

SUSROUTINE SCCT( IBNCH, ELUAL, NEL, NW, I)

DIMENSION ELUAL (2), IBNCHK 7,1 )

C*** THIS SLOFOUTINE REPLACES SC BY GMRATOR AND OC $N 1=I$ BNCHK \&, I) \$N2=IBNCHK 5, I)

$M N=N+1$

IRAOHK $2, I 2=N \mathrm{~N}$

IBNCHK $3, I>0$

IENCK $6, I>1$

ISHCH 7. I > $=0$

ELUALCI $D=1.0$

NEL aNel +1

IBAOK 1, NEL I IBNCK 1, I)

IENCH 2, NEL Y Y N1

IBNCNK 3 , NEL I IN

IEACHK \&, MEL Y YOO

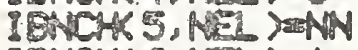

IENCH 6, MEL ) $=1$

IENCH $?$, NEI ) =

ELALSNEL Y 1.0

RETURN

END 
SUBROUTINE TRLINK IBNCH, ELUAL, NEL, I, RQ )

OIMENSION ELURL $(1)$, IBNCHK 7,1$)$

$N I=I B N C H K 4, I) \& N 2=I$ SNCHK $5, I\}$

$N 3=1 B N C H(2, I) \$ N 4=I B N C H K 3, I)$

$I B N C H(2, I)=I H$

IBNCH $3, I)=1 \mathrm{H}$

I

I

EL UAL (I) $=R \theta$

$N E L=K E L+1$

IBNCHK I, NEL I =IENCHK I, I)

IBNCH $2, N$ L $)=1 \mathrm{H}$

IAACHK $3, N E L$ I $=1 H$

IBNDK $4, N E L$ I $=N 2$

IBNOK S, NEI $=N 1$

IBACH $6, N E L$; $=1$

IBNDK ?, NEI $>3$

EL VAL (NEL $=1.0$

$\mathrm{NE}=\mathrm{NEL}+1$

IENCK 1, NE IEIENCHK!, I)

IBNCHK 2, NEL $=1 \mathrm{H}$

IBNCHK $3, N$ E $Y=1 H$

IBNCHK 4, NEL 2=14

IBNCHK S, NEL I $=13$

IBNCH $6, N$ EI $)=!$

IBNCK ?, NEL I 204

ELUA CNEL 2ख1.

$\mathrm{NEL}=\mathrm{NEL}+\mathrm{I}$

IBHCH I, NEL $2=1$ BNCHK 1, I)

IENCHK $2, N F L 2=1 H$

IENCHK 3, NEL VIIH

IENCK 6, NEL JoN3

IENOK S, NFI $2=N 4$

I 4 OH $6, N$, $2=2$

I S

EL WoL $(N E$ I $=R 8$

RETURN

END

SUBROUTINE UCCS(N1,N2,N3,N4, COND, G,NDIM)

DIMENSIOH ESMDIM, 1)

IF (N1.NE. . AND.N3.NE.8) G(N1,N3)=G(N1,N3)+CONO

IF (N2.NE.0.ANO.N3.NE. 8 ) G(N2,N3 $)=G(N 2, N 3)$-COND

IF (N1.NE. . AND.N4.NE.O) G(N1,N4)=G(N1,N4)-COND

IF (N2. WE. O. ANO.N4.NE.Q) G(N2,N4 2EGKN2,N4)+COND

RETURN

END 
SUBROUTINE BNCHKN1, N2, COND, G, NOIM)

DIMENSION GS NDIM, 1)

IF (N1.NE. Q) $G(N 1, N 1)=G(N 1, N 1)+C O N D$

IF (N2.NE. 0$) G(N 2, N 2)=G(N 2, N 2)+C O N D$

IF (N\&.EQ.O. OR.N2.EQ.G) RETURN

$G(N 1, N 2)=G(N 1, N 2)-C D N D \& G(N 2, N 12=G(N 2, N 1)-C O N D$ RETURN

END

SURROUTINE SRCE (N1, N2, SOURCE, C)

OIPENSION C(1)

IF (N1.NE.Q) C (N1) $=C(N 1)-S O U R C E$

IF (N2.NE.O) C(N2)=C(N2)+SOURCE

RETURN

END

FUMCTION NCOOE(NAME)

DIMENSION IREF( 9 )

OATA IRE /1HI, 1HR, 1HO, 1HC, 1HN, 1HU, 1HL, 1HT, 1HE, Co $1 \mp \pm 1,9$

NCOOE:1

IF (NAMR. EQ. IREF(I)) CO TO 2

1 CONTIMLS

NCOOE $=10$

2 RETURN

END 


\section{REFERENCES}

[1] Nahman, M.S. and Riad, S.M., Some physical signals and systems that yield to the homomorphic transformation, Proc. IEEE, in preparation.

[2] Oppenheim, A.V., Schafer, R.W. and Stockham, T.W., Nonlinear filtering of multiplied and convolved signals, Proc. IEEE 56, 1264-1291 (August 1968).

[3] Schafer, R.W., Echo removal by discrete generalized linear filtering, Research Lab. of Electronics, MIT, Tech. Rep. 466 (1969).

[4] Oppenhein, A.V. and Schafer, R.W., Digital Signal Processing, Chs. 2, 3, and 10 (Prentice-Hall, Englewood Cliffs, N.J., 1975).

[5] Ulrych, T.J., Application of homomorphic deconvolution to seismology, Geophysics 36 , 650-660 (August 1971).

[6] Nelson, R.E. and Cozyell, M.R., Electrical parameters of precision, coaxial, airdielectric transmission lines, Nat. Bur. Stand. (U.S.), Monogr. 96 (June 1966).

[7] Riad, S.M. and Nahman, N.S., Application of the homomorphic deconvolution for the separation of IDR signals occurring in overlapping time windows, CPEY Digest, 27-29 (June 1976).

[8] Riad, S.M. and Nahman, N.S., Application of the homomorphic deconvolution for the separation of TDR signals occurring in overlapping time windows, IEEE Irans. Instrum. Yeas., to be published (December 1976).

[9] VonHippel, A.R., Dielectric Materials and Applications (Chapnan and Hall, London; MIT Tech. Press and J. Wiley, 1954).

[10] VonHippel, A.R., Dielectrics and haves (MIT Press, Cambridge, N.Y., 1954).

[11] Hill, N.E., et al., Dielectric Properties and Molecular Behavior (Van Nostrand Reinhold Co., London, 1969).

[12] Cole, R.H., Evaluation of Dielectric Behavior by Time Domain Spectroscopy. II. Complex permittivity, The Journal of Physical Chemistry 79, No. 14, 1469-1474 (1975).

[13] Iskander, M.F. and Stuchly, S.S., A time domain technique for measurement of the dielectric properties of biological substances, IEEE Trans. Instrum. Meas. IM-2I, No. 4, 425-429 (November 1972).

[14] Bucci, 0.M., et al., Time domain techniques for measuring the conductivity and permittivity spectrum of materials, IEEE Trans. Instrum. Meas. IM-21, No. 3, 237-243 (August 1972).

[15] Fellner-Feldegg, H., A thin-sample method for the measurement of permeability, permittivity, and conductivity in the frequency and time domain, Journal of Physical Chemistry 76, No. 15, 2116-2122 (Ju1y 1972).

[16] Nicolson, A.M. and Ross, G.F., Measurement of the intrinsic properties of materials by time-domain techniques, IEEE Trans. Instrum. Meas. IM-19, No. 4, 377-832 (November 1970).

[17] Fellner-Feldegg, H. and Barnett, E.F., Reflection of a voltage step from a section of transmission line filled with a polar dielectric, Journal of Physical Chemistry 74 , No. 9, 1962-1965 (April 1970). 
[18] Fellner-Feldegg, H., The measurement of dielectrics in the time domain, Journal of Physical Chemistry 73, No. 3, 616-623 (March 1969).

[19] Frühlich, H., Theory of Dielectrics (Clarendon Press, Oxford, 1949).

[20] Riad, S.M, and Nahman, N.S., The application of the homomorphic deconvolution to time domain dielectric spectroscopy, J. Phys. Chem., in preparation.

[21] Kasa, I., A circle fitting procedure and its error analysis, IEEE Trans. Instrum. Meas. IM-25, 8-14 (March 1976).

[22] Deadrick, F.J., Miller, E.K. and Hudson, H.G., The LLL transient-electromagneticsmeasurement facility, Lawrence Livermore Laboratory, Livermore, Calif., UCRL-51933 (October 29, 1975).

[23] VanBlaricum, M.L. and Mittra, R., A technique for extracting the poles and residues of a system directly from its transient response, IEEE Trans. Antennas Propagat. AP-23, 777-781 (November 1975).

[24] Tesche, F.M., On the analysis of scattering and antenna problems using the singularity expansion technique, IEEE Trans. Antennas Propagat. AP-21, 53-62 (January 1973).

[25] Hildebrand, F.B., Introduction to Numerical Analysis, Pp. 378-382 (McGraw-Hil1 Book Co., New York, N.Y., 1956).

[26] Ondrejka, A.R., Nat. Bur. Stand., Boulder, Colo., private communication.

[27] Howard, D., Best, A. and Umphrey, J., The wide-band sampling gate: An analysis, chracterization and application discussion, WESCON Tech. Papers, Pt. 6, Session 23, Paper 1 (1966).

[28] Grove, W.M., Sampling for oscilloscopes and other rf systems: dc through x-band, IEEE Trans. Microwave Theory \& Techniques MTT-14, 629-635 (December 1966).

[29] Lennon, C.A., Jr., Measurement of microwave sampling gates, Master's Thesis, University of Toledo (August 1975).

[30] Whinnery, J.R., Jamieson, H.W. and Robbins, T.E., Coaxial lines discontinuities, Proc. IRE 32, 695-709 (November 1944).

[31] Whinnery, J.R. and Jamieson, H.W., Equivalent circuits for discontinuities in transmission lines, Proc. IRE 32, 98-115 (February 1944).

[32] Ramo, S., Whinnery, J.R., and VanDuser, T., Fields and Waves in Communication Electronics, pp. 462-465 (J. Wiley, New York, N.Y., 1965).

[33] Kaposhilin, J.N., Hot carrier diode opens new vistas, Electronic Design, 178-184 (March 1966).

[34] Hewlett Packard Associates, The hot carrier diode theory, design, and application, Application Note, AN 907, Hewlett Packard Co., Palo Alto, Calif.

[35] Chaffin, R.J., Microwave Semiconductor Devices, Ch. 6 (J. Wiley, New York, N.Y., 1973).

[36] Hewlett Packard Co., Time domain reflectometry, Application Note, AN 62 (1964).

[37] Nahman, N.S. and Jickling, R.M., Frequency domain measurements of baseband instrumentation, Nat. Bur. Stand. (U.S.), Internal Report 73-330 (July 1973).

[38] Andrews, J.R., Nat. Bur. Stand., Boulder, Colo., private communication. 
[39] Hewlett Packard Co., 1430A Sampler Operating and Service Manual (February 1967). [40] Riad, S.M. and Nahman, N.S., Transfer network identification (modeling) of feedthrough sampling-head, IEEE Trans. Microwave Theory \& Techniques, in preparation.

[41] Calahan, D.A., Computer-Aided Network Design, Chs. 4 and 9 (McGraw-Hill Book Co., New York, N.Y., 1972).

[42] Branin, F.H., Jr., Transient analysis of lossless transmission lines, Proc. IEEE $\underline{55}$, 2012-2013 (November 1967). 


\begin{tabular}{|c|c|c|c|}
\hline $\begin{array}{l}\text { U.S. DEPT. OF COMM. } \\
\text { BIBLIOCRAPHIC DATA } \\
\text { SHEET }\end{array}$ & $\begin{array}{l}\text { 1. } \mathrm{PL} \text { CATION OR REPORT NO. } \\
\text { NBSIR 78-881 }\end{array}$ & $\begin{array}{l}\text { 2. Gov't Acces., in } \\
\text { No. }\end{array}$ & 3. Recipient's Accession No. \\
\hline \multirow{2}{*}{\multicolumn{3}{|c|}{$\begin{array}{l}\text { "Application of the Homomorphic Transformation } \\
\text { to Time Domain Measurement Problems" }\end{array}$}} & $\begin{array}{l}\text { 5. Publication Date } \\
\text { June } 1978\end{array}$ \\
\hline & & & $\begin{array}{l}\text { 6. Performing Organization Code } \\
724\end{array}$ \\
\hline \multicolumn{3}{|c|}{$\begin{array}{l}\text { 7. AUTHOR(S) } \\
\text { RIAD, SEDKI M. and NAHMAN, NORRIS S. }\end{array}$} & 8. Performing Organ. Report No. \\
\hline \multicolumn{3}{|c|}{ 9. PERFORMING ORGANIZATION NAME AND ADDRESS } & $\begin{array}{l}\text { 10. Project/Task/Work Unir No. } \\
724-4153\end{array}$ \\
\hline \multicolumn{3}{|c|}{$\begin{array}{l}\text { DEPARTMENT OF COMMERCE } \\
\text { Washington, D.C. } 20234\end{array}$} & $\begin{array}{l}\text { 11. Contract/Grant No. } \\
\text { CST- } 8392\end{array}$ \\
\hline \multirow{2}{*}{\multicolumn{3}{|c|}{$\begin{array}{l}\text { 12. Sponsoring Organization Name and Complete Address (Street, City, State, ZIP) } \\
\text { Supported in part by: } \\
\text { Electrical Engineering Department } \\
\text { University of Colorado } \\
\text { Boulder, Colorado }\end{array}$}} & $\begin{array}{l}\text { 13. Type of Report \& Period } \\
\text { Covered }\end{array}$ \\
\hline & & & 14. Sponsoring Agency Code \\
\hline
\end{tabular}

15. SUPPLEMENTARY NOTES

16. ABSTRACT (A 200-word or less factual summary of most significant information. If document includes a significant bibliography or literature survey, mention it here.)

This report presents a study of the theory and application of the homomorphic transformation to deconvolution problems occurring in time domain measurements in the picosecond time domain. A homomorphic deconvolution transform was developed and applied successfully to remove the time-windowing restriction required in many time domain measurements. Examples were presented including problems in time domain analysis of linear networks and dielectric spectroscopy, and scattering and multiple reflection in antenna (radiation) systems were considered and treated. Also considered was the development of a model for a 28 picosecond resolution feedthrough sampling-head, and the model's step response was computed. Simulation studies were performed using typical input waveforms and the oscilloscope model. The homomorphic transformation was used to deconvolve the model's impulse response from the simulated output. Comparison of the deconvolved output waveforms with the input waveforms showed agreement within the accuracy of the sampled-data simulation.

17. KEY WORDS (six to twelve entries; alphabetical order; capitalize only the first letter of the first key word unless a proper name; separated by semicolons) Antenna scattering; deconvolution; dielectric spectroscopy; homomorphic transformation; modeling of sampling gates; signal processing; time domain measurements; time domain reflectometry.
8. AVAILABILITY
(2) Unlimited

For Official Distribution. Do Not Release to NTIS

Order From Sup. of Doc., U.S. Government Printing Office Washington, D.C. $20402, \mathrm{SD}$ Cat. No. C13

X Order From National Technical Information Service (NTIS) Springfield, Virginia 22151

\begin{tabular}{|l|c|}
\hline $\begin{array}{l}\text { 19. SECURITY CLASS } \\
\text { (THIS REPURT) }\end{array}$ & 21. NO. OF PAGES \\
UNCL ASSIFIED & 207 \\
\hline $\begin{array}{l}\text { 20. SECURITY CLASS } \\
\text { (THIS PAGE) }\end{array}$ & 22. Price \\
UNCLASSIFIED & 99.25 \\
\hline
\end{tabular}

